\title{
ESNR 2021
}

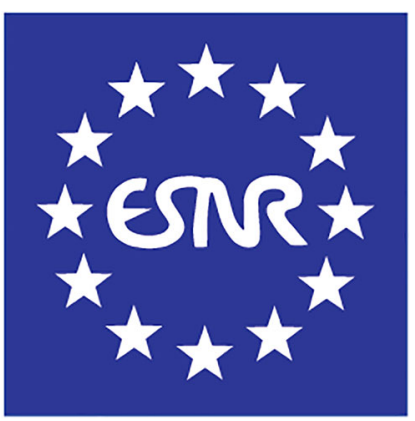

\section{EUROPEAN SOCIETY OF NEURORADIOLOGY}

Diagnostic and Interventional

$44^{\text {th }}$ ANNUAL MEETING

$27^{\text {th }}$ Advanced Course in Diagnostic Neuroradiology

$12^{\text {th }}$ Advanced Course in Interventional Neuroradiology

$29^{\text {th }}$ September $-3^{\text {rd }}$ October 2021

Geneva, Switzerland

Hybrid Meeting

\section{SCIENTIFIC COMMITTEE}

Karl-Olof Lovblad

Paulina Due - Tønnessen

Chen Hoffmann

Naci Koçer

Luigi Manfre

Katarina Surlan Popovic

Meike Vernooij

Pedro Vilela 


\section{ESNR PRESIDENTIAL ADDRESS}

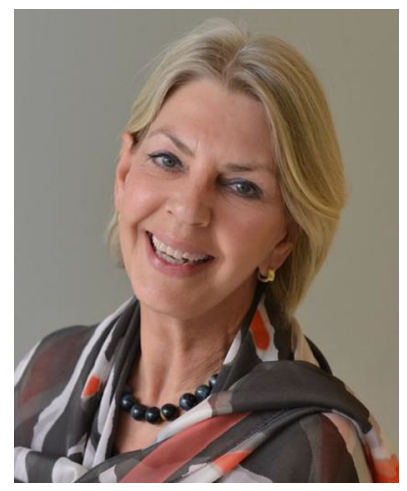

The corona pandemic not only brought grieve to some of us, but also anxiety, economic loss and increased stress to many. Luckily, at the time I write you this welcome address, the world seems to take a turn for the better. Therefore, it is a pleasure that ESNR this year has planned for the Annual Meeting in Geneva. This year's $44^{\text {th }}$ Annual Meeting of the European Society of Neuroradiology (ESNR) will be a very special event in form of a first time exclusive "hybrid meeting" with the possibility for physical attendance at the convention Center in Geneva as well as being able to attend online organized by our Central Office in Switzerland and broadcasted all over the world.

At this 2021 Annual Meeting we will bring you a scientifically and educational meeting as in the old times but also with the hybrid version we decided for a significantly reduced registration fee for all participants. The ESNR Annual Meeting will start with a 7T Symposium on Wednesday $29^{\text {th }}$ September, followed by the Advanced Courses in Diagnostic and Interventional Neuroradiology on Thursday $30^{\text {th }}$ September. It will continue with State-of-the-Art, educational and scientific lectures and presentations until $3^{\text {th }}$ of October. The presentations of the Honorary Members and Awards Ceremony will occur on Friday $1^{\text {st }}$ of October, and the General Assembly on Saturday October $2^{\text {nd }}$. We were able to put together a very interesting program brought to you for a very reasonable price. State-of-the-Art lectures, review courses and scientific presentations will be brought live as well as online to you every day during the Annual Meeting with support from our Central Office and our ESNR Congress President Prof. Karl-Olof Lövblad.

During the Annual Meeting we will celebrate Prof. Dr. med. Rüdiger von Kummer and Prof. Nadine Girard as Honorary Members for their outstanding contributions to neuroradiology during their long and successful professional careers. In addition we will acknowledge our honorary members from 2020 Professors Leon van Rensburg and Zoltan Patay.

We can't make up for the lack of social gathering, networking and meeting friends over the past year but I do hope that this "hybrid" ESNR Annual Meeting" will give us the possibility to meet in person again. For those of us that cannot attend in person our hybrid version will give you the opportunity to join and interact online. I am looking forward to meeting you all.

Let us make this annual meeting great both scientifically, educational, socially, and let us hope this is a turning point that the world will resume to normal in near future.

Prof. Pia C Maly Sundgren

ESNR President 


\section{WELCOME ADDRESS}

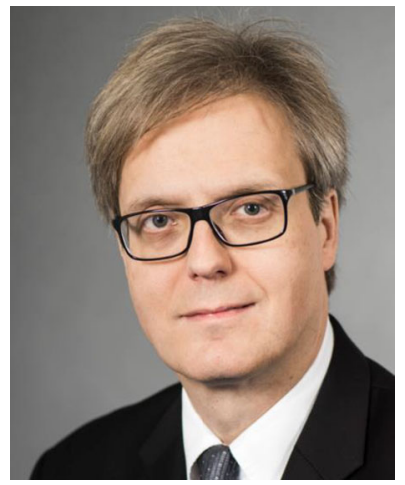

It is our pleasure and privilege to welcome you to Geneva, Switzerland for the 44th Annual Meeting of the ESNR and the 27th Advanced Course in Diagnostic Neuroradiology as well as the 12th Advanced Course in Interventional Neuroradiology.

Besides covering all the varied aspects of modern neuroradiology, the Congress aims to address modern new issues facing the radiological sciences such as the emergence of Artificial Intelligence in imaging, new socio-economic aspects of radiology as well as trends in teleradiology and interventional neuroradiology.

The ESNR Scientific Committee has developed an appealing programme that will be of both interest for neuroradiologists-in-training and for continuing education purposes. An international faculty of well-established expert speakers has been chosen in order to provide the optimal learning experience for all attendees.

The diagnostic course will deal with topics such as techniques and indications in emergency neuroradiology, as well as addressing new imaging findings observed during the covid pandemic. The interventional course will deal with the latest developments in the endovascular treatment of stroke and aneurysms. A half-day pre-course will deal with new developments in ultra-high-field imaging.

Geneva, while lying at the border of Europe, is a major hub for international organizations and academia. The meeting will take place at the Centre International des Conferences de Genève (CICG), which lies in the heart of the city's International Center: close to the United Nation and other international organizations. The congress venue is easily joinable in a few minutes by public transportation from the city center and the airport.

This is the fourth time that Switzerland, and the second time that Geneva, welcomes the ESNR and its congress. This is a recognition of Swiss neuroradiology both nationally and internationally.

We hope that you will have as much pleasure attending the conference as we did in preparing it.

\section{Karl-Olof Lövblad}

Congress President, $44^{\text {th }}$ ESNR Annual Meeting 
Executive Committee

President

Vice-President

Secretary General

Treasurer

Chairperson of the Subspecialty Committee Diagnostic Neuroradiology

Chairperson of the Subspecialty Committee Head and Neck Neuroradiology

Chairperson of the Subspecialty Committee Interventional Neuroradiology

Chairperson of the Subspecialty Committee Pediatric Neuroradiology

Chairperson of the Subspecialty Committee Spine Neuroradiology

Secretary of the Institutional Council

Institutional Council

Institutional Members

Australian and New Zealand Society of Neuroradiology

Austrian Society of Neuroradiology

Belgian Society of Neuroradiology

Brazilian Society of Neuroradiology

British Society of Neuroradiologists

Bulgarian Society of Neuroradiology \& Head and Neck Radiology

Danish Society of Neuroradiology

Dutch Society of Neuroradiology

Finnish Society of Neuroradiology

French Society of Neuroradiology

German Society of Neuroradiology

Greek Society of Neuroradiology

Gulf Neuroradiology Society

Hungarian Society of Neuroradiology

Irish Society of Neuroradiology

Italian Society of Neuroradiology

Latvian Society of Neuroradiology

Neuroradiology Section of the Association of Radiologists of Ukraine

Neuroradiology Section of the Association of Radiology of Bosnia \& Herzegovina

Neuroradiology Section of the Croatian Society of Radiology

Neuroradiology Subgroup of the Radiological Society of South Africa

Norwegian Society of Neuroradiology

Polish Medical Society of Radiology - neuroradiology section

Portuguese Society of Neuroradiology

Serbian Society of Neuroradiology

Slovenian Section of Neuroradiology

Spanish Society of Neuroradiology

Swedish Society of Neuroradiology

Swiss Society of Neuroradiology

Turkish Society of Neuroradiology
Pia Maly Sundgren

Tarek Yousry

Andrea Rossi

Cem Calli

Meike Vernooij

Katarina Surlan Popovic

Naci Koçer

Chen Hoffmann

Luigi Manfrè

Paulina Due-Tønnessen 
Committees of the ESNR

\begin{tabular}{|c|c|c|}
\hline Diagnostic Neuroradiology & & G. Bonte \\
\hline Meike Vernooij (Chair) & Scientific Programme & J. Bories \\
\hline Alexandre Krainik & K. O. Lövblad (Chair) & G. Bradac \\
\hline \multirow[t]{2}{*}{ Luc van den Hauwe } & Meike Vernooij & J.P. Braun \\
\hline & Naci Koçer & P. Buffard \\
\hline Ana Ramos & Katarina Surlan Popovic & J.W.D. Bull \\
\hline \multirow[t]{2}{*}{ Antonella Castellano } & Chen Hoffmann & E.H. Burrows \\
\hline & Luigi Manfrè & A. Calabrò \\
\hline Head and Neck Neuroradiology & & R. Chrzanowski \\
\hline Katarina Surlan Popovic (Chair) & P. Vilela & G. Cornélis \\
\hline Sotirios Bisdas & Paulina Due-Tønnessen & S. Cronqvist \\
\hline Bernhard Schuknecht & & K. Decker \\
\hline \multirow[t]{2}{*}{ Sofie Van Cauter } & Membership & P. Dettori \\
\hline & Cem Calli (Chair) & R. Djindjian \\
\hline Interventional Neuroradiology & Paulina Due-Tonnessen & A. Donaldson \\
\hline Naci Koçer (Chair) & Zulejha Merhemic & G. du Boulay \\
\hline Omer Eker & Martina Špero & J. Duquesnel \\
\hline Markus Möhlenbruch & & $\mathrm{Ph}$. Engel \\
\hline Iș1l Saatci & Social Media & L. Escudero \\
\hline \multirow[t]{2}{*}{ Kamil Zelenak } & Gennaro D’Anna (Chair) & H. Fischgold \\
\hline & Efrosini Papadaki & T. Greitz \\
\hline Pediatric Neuroradiology & Julia Fruehwald-Pallamar & G. Gryspeerdt \\
\hline Chen Hoffmann (Chair) & Yelda Ozsunar Dayanir & H. Hacker, Germany \\
\hline Maria Argyropoulou & Chiara Gaudino & D.T. Hawkins \\
\hline Kshitij Mankad & Johan Van Goethem & R. Hoare \\
\hline \multirow[t]{2}{*}{ Mariasavina Severino } & Luc van den Hauwe & P. Huber \\
\hline & Nadya Pyatigorskaya & H.H. Jacobsen \\
\hline Rules & Rudiger Von Kummer & J. Jeanmart \\
\hline Philippe Demaerel (Chair) & & J. Jirout \\
\hline Andrea Rossi & ESONR & E.M. Klausberger \\
\hline Marek Sasiadiek & Johan Van Goethem (Chair) & J. Legré \\
\hline \multirow[t]{2}{*}{ Dojan Stojanov } & Luigi Manfrè & B. Liliequist \\
\hline & Naci Koçer & E. Lindgren \\
\hline Spine Neuroradiology & Chen Hoffmann & G. Lombardi \\
\hline Luigi Manfrè (Chair) & Katarina Surlan Popovic & J. Metzger \\
\hline Adrian Kastler & Meike Vernooij & P. Moxon \\
\hline Chiara Zini & Majda M. Thurnher & D. Müller \\
\hline Inês Sofia Martins Gil de Almeida & & R. Oberson \\
\hline Hosam Alqatami & Founding Members & A. Passerini \\
\hline Allan Brook & & L. Penning \\
\hline Bassem Georgy & P. Amundsen & J. Petrov \\
\hline Wende Gibbs & P. Andersen & L. Picard \\
\hline \multirow[t]{2}{*}{ Joshua Hirsch } & H. Backmund & P. Potthoff \\
\hline & K. Bergström & L. Psenner \\
\hline Rules & V. Bernasconi & R. Reid \\
\hline Philippe Demaerel (Chair) & A.S. Bligh & J. Roulleau \\
\hline \multicolumn{3}{|l|}{ Andrea Rossi } \\
\hline \multicolumn{3}{|l|}{ Marek Sasiadiek } \\
\hline Dojan Stojanov & & \\
\hline
\end{tabular}




\section{THE ESNR AWARDS: \\ THE EUROPEAN NEURORADIOLOGY AWARDS IN DIAGNOSTIC AND INTERVENTIONAL NEURORADIOLOGY}

\section{ESNR Awards}

The European Society of Neuroradiology (ESNR) awards three Annual Scientific Prizes for the best works in Neuroradiology, each to the amount of $€ 4000$ :

The "ESNR Scientific Award (formerly known as the "Lucien Appel Prize of the ESNR")", to be awarded for the best Research submission. The "Pioneers and Past Presidents of European Neuroradiology Awards", for the best submissions on Diagnostic Neuroradiology.

The "Pioneers and Past Presidents of European Neuroradiology Awards", for the best submissions on Interventional Neuroradiology. The "ESNR-Springer Award" will be awarded by Springer to the firstnamed author (ESNR Member) of the most cited article of the latest impact factor together with the prize money of $€ 1000$ and a free registration to the ESNR Annual Meeting of the current year. The Article will be highlighted on the journal webpage (Springer.com)

\section{Rules of participation for ESNR Awards:}

Article 1: The prizes reward the achievements of young scientists, under the age of 40 years on the day of application, working in the field of neuroradiology in a European centre and beeing Full, Associate or Junior Member of the ESNR (can be pending or active).

Article 2: The prizes, each to the amount of $€ 4000$, are awarded every year at the Awards Ceremony during the annual congress of the ESNR, or during the Symposium Neuroradiologicum when the ESNR Annual Congress does not take place. The Awards Ceremony is a respected event during each congress; other simultaneous ESNR sessions are not allowed, and the ceremony may not be split in two or more parts. The Award Ceremony is conducted by the Chair of the Awards Committee, who also introduces the awardees and presents the prizes together with the ESNR President.

Article 3: The candidate must be the first author of an original work in the fields of research in neuroradiology: diagnostic or interventional neuroradiology. Applications can be placed for a scientific work that was: a. published online or in print in a peer-reviewed scientific journal listed in SCI within the last 12 months before the date of submission, or b. submitted, still unpublished, accepted or not yet accepted. The candidate must not have received any other prize for the same work, and must not have received the ESNR award of the same category previously.

Article 4: All submissions must be in English and should include:

- application letter indicating the title of the scientific paper upon which the application is based, and reporting its status if not yet published;

- the scientific paper (unpublished or to be submitted);

- the full Curriculum Vitae and a photo of the candidate.

These documents must be exclusively submitted, as electronic attachments, via e-mail to the address indicated below. Please note that each submitted article and its relevant images should be included in a separate attachment.

By submitting the work the author agrees, if declared the winner, to present the work at the next ESNR Annual Congress or Symposium
Neuroradiologicum. Should the winner be unable to participate because of proven urgent reasons, the presentation can be made by a co-author of the same paper. If no presentation is made, the prize money will not be awarded. Payment of the prize money is made after the relevant ESNR Annual Congress or Symposium Neuroradiologicum has taken place.

Article 5: A single author can apply for more than one prize with scientific papers on clearly different topics as confirmed by the Awards Committee.

Article 6: Authors are requested to indicate to which of the two awards categories each scientific paper is addressed. If the Awards Committee considers another category to be more appropriate, the submission may be re-classified.

Article 7: A Scientific Jury will assess and rate the submitted papers. The Jury is composed of the members of the Awards Committee, plus four internationally known specialists in neuroradiology, who will rate each application independently from each other. If the rating does not give clear results, the prize-winners will be identified by consensus of all jury members.

The members of the Scientific Jury should declare not to have a conflict of interests. In case of conflict of interests, the juror is excluded from the Scientific Jury for the current year and specific prize category, and is replaced by another juror of equal scientific standing.

The Chair of the Awards Committee shall be invited as a Faculty member to the ESNR Annual Meeting or Symposium Neuroradiologicum where the award ceremony takes place.

Article 9: The ESNR will advertise the prizes by a dedicated newsletter and in the society website, as of January $1^{\text {st }}$ each year. The deadline for applications is set at March $1^{\text {st }}$ each year. The Award Committee will designate the laureates after receiving the evaluations of the jurors, as a rule by May $1^{\text {st }}$. Should the Award Committee decide that, in any category, no submission meets sufficientquality requirements, no prize will be awarded in that category.

Article 10: All candidates will be notified in writing of the result of their application. The laureates will be invited to give a six-minute oral presentation at the Award Ceremony during the ESNR Annual Congress, or Symposium Neuroradiologicum when the ESNR Annual Congress does not take place. The congress registration fee of the laureates is waived; the travel expenses will be reimbursed.

The names of the laureates, as well as the titles and abstracts of their scientific works, will be placed on the ESNR website and published in the society pages of Neuroradiology. In case the winning paper is still unpublished, the authors agree that "Neuroradiology", the official ESNR journal, shall have the right of publication after peer-review.

Article 11: All possible questions and issues regarding the awarding of the Prizes shall be dealt with at the discretion of the Awards Committee and the ESNR Executive Committee. The decision of the ESNR Executive Committee is final.

Article 12: Candidacy implies full acceptance of the rules. Please send your submissions to the attention of the ESNR Secretary GeneralEmail: info@esnr.org

The object of the email should read: ESNR award submission. 


\section{THE DEADLINE FOR RECEIPT OF SUBMISSIONS IS MARCH $1^{\text {ST }}$}

ESNR Annual Congress, Advanced Course in Diagnostic Neuroradiology and Advanced Course in Interventional Neuroradiology of the ESNR.

The European Society of Neuroradiology holds each year a scientific congress at a place and date designated by the Executive Committee. On the day before the congress, Advanced Courses in Diagnostic Neuroradiology and Interventional Neuroradiology are organized in parallel sessions. The business meeting (General Assembly) of the Society is held in conjunction with this annual scientific congress.

Since its creation in 1969, the ESNR has organized 42 annual congresses. In recognition of the importance of the Symposium Neuroradiologicum, which is held every four years, no scientific congress of the ESNR is held when the Symposium takes place in Europe.

\section{Future Annual Meetings}

$4^{\text {th }}$ Annual Meeting

$15^{\text {th }}-18^{\text {th }}$ September 2022

Lisbon, Portugal

President: Pedro Vilela

\section{$4^{\text {th }}$ Annual Meeting}

September 2023

Vienna, Austria

President: Majda Thurnher

\section{The European Course in Neuroradiology}

The European Course in Neuroradiology has been a story of success ever since the first course in Toulouse in 1984. More than 1000 young neuroradiologists have completed the cycle of three courses over the past 21 years. The ECNR was conceived as a means to create a common and shared culture, common meeting points and a common standard of knowledge. The form that was adopted and used for the coming 6 cycles, each consisting of three courses, included the major bodies of knowledge as described by the headlines; intracranial nervous system, the spine and spinal cord and the base of the skull, maxillofacial and head and neck neuroradiology.

\section{European School of Neuroradiology (ESONR) - the new concept}

The ESONR - European School of Neuroradiology - is the most important and complete training programme organized by the ESNR - European Society of Neuroradiology within the main frame, and in partnership with, the ESOR - European School of Radiology. The purpose of this training and education program is based on the vision of what the ESNR considers the range of competences and skills that are the basis of a high qualified neuroradiological activity. The program is designed to offer a pathway to reach such professional and cultural levels. The final points will be the examinations to be certificated at the different levels and in the different branches of this discipline.

Courses are organized at 3 different levels: 1st Level: ESOR - Galen Foundation Courses in Neuroradiology, 2nd level: ECNR - Course in Neuroradiology, Diagnostic and Interventional and finally 3rd level: ESNR Advanced Courses of Higher Qualification in Interventional Endovascular Neuroradiology, Interventional Spine Neuroradiology, Advanced Diagnostic Neuroradiology and Paediatric Neuroradiology.
ECNR - European Course in Diagnostic and Interventional Neuroradiology

The ECNR is the fundamental Neuroradiology course aimed at neuroradiologists, established or in training. It is based on cycles of four courses (modules), each lasting five days, dedicated to diagnostic and interventional neuroradiology. The full cycle is considered complete after the attendee has participated in all four modules, which can be done in a single cycle or in different cycles.

The scientific content of the course is determined by the ESONR Committee of the European Society of Neuroradiology, taking into account international standards and guidelines for training in diagnostic and interventional neuroradiology. The participating educators will be internationally renowned European experts, to be selected on the basis of their scientific background and educational skills to ensure high- quality lectures and interactive case discussions.

The following topics have been chosen, each to be covered in five full days of lectures and workshops:

- Anatomy, congenital malformations and genetics.

- Trauma, Infection, Inflammation and Degenerative Disease

- Tumours of the Brain and Spinal Canal

- Vascular Disease of the Brain and Spinal Canal

Seventh Cycle - Scientific Director: Guido Wilms (Leuven)

First Module: Embryology, Anatomy, Malformations - Crieff, October 4 -11, 2002 -Local Director:Wendy J. Taylor

Second Module: Tumours of the CNS - Riga, April 11 - 18, 2003 - Local Director: Cosma Andreula

Third Module: Vascular Disease of the CNS - Malta, October, 24 - 30, 2003 - Local Director: Athanassios Gouliamos

Fourth Module: Trauma and Degenerative Disease of the CNS - Riga, April 23-27, 2004 -Local Director: Johan Van Goethem

Eighth Cycle -Scientific Director: Ernst-Wilhelm Radü (Basel) First Module: Embryology/Anatomy/Malformations/Genetics - Basel, October 22-26, 2004

Second Module: Tumors of the CNS - Basel, March 18-22, 2005

Third Module: Vascular Diseases of the CNS - Basel, October 21-25, 2005

Fourth Module: Trauma/Degenerative Diseases of the CNS - Basel, March 10-14, 2006

Ninth Cycle - Scientific Director: Ernst-Wilhelm Radü (Basel)

First Module: Embryology/Anatomy/Malformations/Genetics - Basel, October 20 - 24, 2006

Second Module: Tumours of the CNS - Basel, March 23 - 27, 2007

Third Module: Vascular Diseases of the CNS - Basel, November 2 - 6, 2007

Fourth Module: Trauma/Degenerative Diseases of the CNS - Basel, April $11-15,2008$

Tenth Cycle - Scientific Directors: Massimo Gallucci (L'Aquila), AlexRovira (Barcelona)

First Module: Embryology/Anatomy/Malformations/Genetics Tarragona, October $10-14,2008$

Second Module: Tumors and Tumor-like Vascular Lesions- Rome, March 20 - 24, 2009

Third Module: Vascular Diseases - Tarragona, October 9-13, 2009

Fourth Module: Trauma/Degenerative/Metabolic/Inflammatory Diseases - Rome, March 19-23, 2010

Eleventh Cycle - Scientific Directors: Massimo Gallucci (L'Aquila), Alex Rovira (Barcelona)

First Module: Embryology/Anatomy/Malformations/Genetics Tarragona, November 5-9, 2010

Second Module: Tumors and Tumor-like Vascular Lesions- Rome, March 25-29, 2011 
Third Module: Vascular Diseases - Tarragona, October 28 - November 1, 2011 Fourth Module: Trauma/Degenerative/Metabolic/Inflammatory Diseases-Milano, April 12-16, 2012

Twelfth Cycle - Scientific Directors: Athanasios Gouliamos (Athens), E. Turgut Tali (Ankara)

First Module: Embryology/Anatomy/Development and Malformations of the CNS - Antalya, November 1-6, 2012

Second Module: CNS Tumors - Athens, April 8 - 13, 2013

Third Module: Vascular Diseases - Antalya, November 3-7, 2013

Fourth Module: Trauma/Degenerative/Metabolic/Inflammatory Diseases - Athens, April6-10, 2014

Thirteenth Cycle - Scientific Directors: Athanasios Gouliamos (Athens), E. Turgut Tali (Ankara)

First Module: Embryology/Anatomy/Development and Malformations of the CNS - Antalya, November 9-13, 2014

Second Module: CNS Tumors - Rhodes, April 14-18, 2015

Third Module: Vascular Diseases - Izmir, November, 2015

Fourth Module: Trauma/Degenerative/Metabolic/Inflammatory Diseases

- Rhodes, April, 2016

Fourteenth Cycle - Scientific Directors: Majda M. Thurnher (Vienna), Johan Van Goethem (Antwerp)

First Module: Embryology/Anatomy/Development and Malformations of the CNS - Dubrovnik, October 16-20, 2016

Second Module: CNS Tumors - Antwerp, May 7-11, 2017

Third Module: Vascular Diseases - Dubrovnik, October 22-26, 2017

Fourth Module: Trauma, Infection and Degenerative Disease - Antwerp, May 6-10, 2018
Fifteenth Cycle - Scientific Directors: Majda M. Thurnher (Vienna), Johan Van Goethem (Antwerp)

First Module: Anatomy and Embryology - Dubrovnik, October 21-25, 2018

Second Module: CNS Tumors - Antwerp, April 29-May 3, 2019

Third Module: Vascular and Traumatic Diseases - Rovinj, 13 - 17 October 2019

Fourth Module: Degenerative, Metabolic and Inflammatory Diseases, Live Online Edition, 8-12 November 2020

\section{Neuroradiology}

Neuroradiology, published by Springer Verlag, was founded as the official organ of the European Society of Neuroradiology in 1970. Since 2010 on, the Austrian Society of Neuroradiology, the Belgian Society of Neuroradiology, the British Society of Neuroradiologists, the Bulgarian Association of Radiology, the Czech Neuroradiological Society, the Dutch Society of Neuroradiology, the Finnish Society of Neuroradiology, the German Society of Neuroradiology, the Latvian Society of Neuroradiology, the Norwegian Society of Neuroradiology, the Neuroradiological Section of the Hellenic Radiological Society, the Neuroradiological Section of the Polish Radiological Society, the Portugese Society of Neuroradiology, the Romanian Society of Magnetic Resonance in Medicine, the Spanish Society of Neuroradiology, the Swedish Society of Neuroradiology and the Swiss Society of Neuroradiology decided to choose neuroradiology as their (in some cases second) official Journal.

Submissions of articles can be done via the website: http:/ www.editorialmanager.com/nrad 
NEURORADIOLOGY

EDITORIAL BOARD 2021

\section{Editor-in-Chief}

R. von Kummer, Dresden

\section{Managing Editor}

L. Babinec, Seattle

Diagnostic Neuroradiology

F. Barkhof, Amsterdam/London (Section Editor)

O. Abe, Tokyo

J.R. Bapuraj, Ann Abor

A.J. Bastos-Leite, Porto

F. Bonneville, Toulouse

B. Brea-Álvarez, Madrid

M.A. Brockmann, Mainz

S.H. Choi, Seoul

E. Gizewski, Innsbruck

R. Gupta, Haryana

M. Hori, Tokyo

S.C. Jung, Seoul

S. Kakeda, Hirosaki

H.S. Kim, Seoul

K. Kudo, Sapporo

A. Kunimatsu, Tokyo

D.S. Liebeskind, Los Angeles

J. Linn, Dresden

M. Maeda, Tsu

F. Meijer, Nijmengen

A. Rovira Cañellas, Barcelona

T. Struffert, Erlangen

T. Taoka, Nagoya

M. Thurnher, Vienna

H. Urbach, Freiburg

P. Valsasina, Milan

M. Vernooij, Rotterdam

Y. Watanabe, Osaka

M. Wattjes, Hannover

G. Wilms, Leuven

M. Wintermark, Stanford

A. Yogi, Okinawa

T.J. Yun, Seoul

Advanced Imaging

S. Haller, Geneva (Section Editor)

C. Benjamin, New Haven

A. Choe, Baltimore

C. Federau, Zürich

Y. Liu, Beijing

K. Lövblad, Geneva

S. Van Cauter, LimburgJ. Wardlaw, Edinburgh

Head-Neck- ENT Radiology

J. Casselman, Brugge (Section Editor)

T. Beale, London

B. De Foer, Wilrijk

N. Hosten, Greifswald

C. Kenis, Heusden-Zolder

M. Lell, Nuernberg

K. S. Popovič, Ljubljana

B. Schuknecht, Zurich

D. Vanneste, Geel
Interventional Neuroradiology

I. Saatci, Ankara (Section Editor)

I. Wanke, Hirslanden (Deputy Editor)

S. Cekirge, Ankara

R. Chapot, Essen

F. Clarençon, Paris

G. Gál, Odense

J. Gerber, Dresden

P. Gölitz, Erlangen

M. Goyal, Calgary

H. Henkes, Stuttgart

N. Koçer, Istanbul

A. Krajina, Hradec Králové

W. Küker, Oxford

W. Kurre, Passau

S. Meckel, Freiburg

D. Meila, Hannover

J. Mocco, New York

M.A. Möhlenbruch, Heidelberg

D.C. Suh, Seoul

B. Tomandl, Göppingen

B. Turowski, Düsseldorf

W. van Zwam, Maastricht

A. Wakhloo, Burlington

Paediatric Neuroradiology

A. Rossi, Genoa (Section Editor)

M. Argyropoulou, Ioannina

F. D'Arco, London

B. Ertl-Wagner, Toronto

C. Hoffmann, Tel Hashomer

P.-L. Khong, Hong Kong

A. McKinney, Minneapolis

Z. Patay, Memphis

A. Righini, Milan

B.P. Soares, Burlington

P.C. Sundgren, Lund

Spinal Neuroradiology

M. Muto, Naples (Section Editor)

A. Brook, Bronx

A. Cianfoni, Lugano

W. N. Gibbs, Phoenix

A. Kastler, Grenoble

L. Manfrè, Catania

A. Romano, Rome

L. Shah, Salt Lake City

J. Van Goethem, Antwerpen

M.-A. Weber, Rostock

Japan

T. Hirai, Kumamoto (Section Editor, Diagnostic)

T. Yoshiura, Kagoshima (Deputy Editor, Diagnostic)

H. Kiyosue, Oita (Section Editor, Interventional)

Statistical Consultants

J. Birks, Oxford

E. Bluhmki, Biberach a.d. RißP. Dupont, Leuven

ESNRG. D'Anna, Legnano 


\section{SCIENTIFIC PROGRAM}

WEDNESDAY $29^{\text {th }}$ September 2021 - Pre-meeting course: 7T symposium

Moderation:

$\begin{array}{ll}\text { 13:30-14:00 } & \text { Physics principles of UHF and future technical developments } \\ & \text { Daniel Nanz, } C H \\ \text { 14:00-14:30 } & \text { MRI safety issues at 7T } \\ & \text { Boel Hansson, } S E \\ \text { 14:30-15:00 } & \text { Vascular applications of UHF systems } \\ & \text { Javier Romero, US } \\ \text { 15:00-15:30 } & \text { Coffee Break } \\ \text { 15:30-16:00 } & \text { Epilepsy and UHF } \\ & \text { Maria Isabel Vargas, } C H \\ \text { 16:00-16:30 } & \text { Spine, present and future } \\ & \text { TBC } \\ \text { 16:30-17:00 } & \text { Clinical future applications } \\ & \text { Elke Gizewski, AT }\end{array}$

THURSDAY 30 ${ }^{\text {th }}$ September 2021 - Meeting room 1

\section{$27^{\text {TH }}$ ADVANCED COURSE IN DIAGNOSTIC NEURORADIOLOGY}

Emergencies in Neuroradiology

08:00-10:00: Techniques and indications

Moderators: Meike Vernooij NL

08:00-08:10 Introduction to the advanced diagnostic course

Karl-Olof Lövblad, CH

08:10-08:30

Which imaging techniques to use for what indication?

Gennaro D'Anna, IT

0830-08:50

Clinical indications of emergency Dual energy CT in neuroradiology

Sebastian Winklhofer, $\mathrm{CH}$

08:50-09:10

Enhancing lesions in emergency situations

09:10-09:30

Jose Boto, $\mathrm{CH}$

0.10-09:30

Diagnostic Errors in emergency imaging

09:30-09:50

Christoph $\mathrm{Ozdoba}, \mathrm{CH}$

10:00-10:30

What the interventional neuroradiologist needs to know from the diagnostic neuroradiologist?

Fabrice Bonneville, FR

10:30-12:00

Coffee Break

Indications II

Moderators: Zulejha Merhemic, BA

10:30-10:45:

10:45-11:05

11:05-11:20

$11: 20-11: 40$

11:40-12:00

12:10 - 13:15

13:30-14:00

14:00-15:30
Imaging in stroke: CT/MR/DSA?

Karl-Olof Lövblad, CH

Pediatric neuro emergencies

Melike Guryildirim, US

Emergency imaging in adult headache

Martha Drake, ES

emergency spine imaging

Johan Van Goethem, BE

Stroke Mimics

Majda Thurnher, AT

Industry Sponsored Symposia

Keynote lecture: Neuroradiology and medicine in the new economic environment

SW Atlas, US

Emergencies III 
Moderators :

\begin{tabular}{|c|c|}
\hline 14:00-14:20 & $\begin{array}{l}\mathrm{AI} \text { in emergency Imaging } \\
\text { Jeremy Hofmeister, } \mathrm{CH}\end{array}$ \\
\hline 14:20-14:40 & $\begin{array}{l}\text { Diagnostic strategies in Intracerebral hemorrhage } \\
\text { Javier Romero, US }\end{array}$ \\
\hline $14: 40-15.00$ & $\begin{array}{l}\text { Acute inflammatory diseases of the brain } \\
\text { Tarek Yousry, UK }\end{array}$ \\
\hline $15: 00-15: 20$ & $\begin{array}{l}\text { Imaging of cranio-cervical trauma. } \\
\text { Cem Calli, TR }\end{array}$ \\
\hline $15: 30-16: 00$ & Coffee Break \\
\hline 16:00-18:00: & Imaging of Neuro-COVID \\
\hline \multicolumn{2}{|l|}{ Moderators : } \\
\hline 16:00-16:30 & $\begin{array}{l}\text { Virologist view of the pandemic } \\
\text { Samira Kremer, FR }\end{array}$ \\
\hline $16: 30-16: 50$ & $\begin{array}{l}\text { Neurological manifestations of COVID-19 } \\
\text { Frederic Assal, } \mathrm{CH}\end{array}$ \\
\hline $16: 50-17: 10$ & $\begin{array}{l}\text { Non stroke patters in neuro-COVID } \\
\text { Stephane Kremer, FR }\end{array}$ \\
\hline $17: 10-17: 30$ & $\begin{array}{l}\text { Stroke and COVID } \\
\text { Rolf Jäger, UK }\end{array}$ \\
\hline $17: 30-17: 50$ & $\begin{array}{l}\text { Thrombectomy and COVID } \\
\text { Basile Kerleroux, FR }\end{array}$ \\
\hline
\end{tabular}

THURSDAY 30 ${ }^{\text {th }}$ September 2021 - Meeting room 2

\section{$12^{\text {TH }}$ ADVANCED COURSE IN INTERVENTIONAL NEURORADIOLOGY}

Interventional neuroradiology of stroke

08:00-10:00: Stroke I: techniques and indications

Moderators

08:00-08:10

08:10-08:30

08:30-09:00

09:00-09:30

09:30-10:00

10:00-10:30

10:30-12:00

Moderators:

10:30-10:50

10:50-11:10

$11: 10-11: 30$

$11: 30-11: 50$

12:10 - 13:15

13:30-14:00

14:00-15:30
Introduction to the interventional course

Paolo Machi, $\mathrm{CH}$

Patient Selection for ischemic stroke treatment.

T. Ullberg, SE

Indication to mechanical thrombectomy.

Birgitta Ramgren, SE

Imaging of penumbra and extended time windows for treatment. Time is brain, but each brain has its time.

Jens Fiehler, DE

Direct aspiration: state of the art.

Marc Ribo, ES

Coffee Break

Stroke II

Combined techniques for thrombectomy.

Paolo Machi, $\mathrm{CH}$

Posterior circulation stroke endovascular treatment

Zsolt Kulcsàr, $\mathrm{CH}$

Complication in mechanical thrombectomy

TBC

Adjunctive stenting during MT in acute large vessel occlusion: When and how

Vitor Mendes Pereira, CA

Industry Sponsored Symposia

Keynote lecture: Neuroradiology and medicine in the new economic environment

SW Atlas, US

Aneurysms 
Moderators:

14:00-14:20 Do not touch aneurysms for endovascular treatment? Where is the current boundary?

14:20-14:40 Would coiling survive in the next decade?

14:40-15.00 Fusiform vertebrobasilar aneurysms:

Treat or not treat? When and how?

Civan Işlak, TR

$15: 00-15: 20$

15:30 - 16:00

16:00-18:00:

What are the results of surgical clipping in the current practice?

Raphael Guzman, $\mathrm{CH}$

Coffee Break

Aneurysms II

Moderators :

$16: 00-16: 30$

$16: 30-17: 00$

The concept of flow remodelling in endovascular aneurysm treatment resulted in a new aneurysm occlusion classification

Saruhan Çekirge, TR

Tips, tricks and pitfalls in the endovascular aneurysm treatment in the acute SAH?

Marios Psychogios, $\mathrm{CH}$

17:00-17:30

Endovascular treatment of ruptured aneurysms: Bail-out strategies with antiplatelet drugs

Omer Eker, FR

17:30-18:00

Vasospasm in acute SAH patients: Follow-up, medical treatment, indications, and techniques of intervention. $T B C$

FRIDAY $1^{\text {st }}$ October 2021 - Meeting room 1

08:00-08:30

08:30 - 10:00

Moderators:

$08: 30-08: 50$

$08: 50-09: 10$

09:10 - 09:30

09:30-09:50

10:00-10:30

10:30-12:00

12:10 - 13:15

13:30-14:00

14:00 - 15:30

Moderators:

$14: 00-14: 20$

$14: 20-14: 40$

$14: 40-15: 00$

$15: 00-15: 20$

15:30 - 16:00

16:00 - 16:30
Interactive cases Diagnostic NR

Luc van den Hauwe, BE

Diag NR: Brain tumor imaging: joint EANO-ESNR session on radiomics/ radiogenomics in clinical neurooncology practice and trial management

What is the value of radiomics in neuro-oncology practice and in new molecular treatments?

$T B C$

MRI-based radiomics signatures: more than meets the eye

Sotiros Bisdas, UK

Radiogenomic approaches and advances: towards a virtual biopsy?

Marion Smits, $N L$

Roundtable on brain tumor imaging:

Pia Sundgren, Marion Smits, Sotiros Bisdas

Coffee Break

Opening Ceremony, Awards Ceremony, Honorary Members Ceremony

Industry Sponsored Symposia

KEYNOTE LECTURE: Tele-expertise and tele-radiology in international activity,

A. Geissbuhler, $\mathrm{CH}$

Diag NR: Epilepsy imaging (surgical planning)

The preoperative work-up in epilepsy surgery

Raphael Guzman, CH

Imaging in the preoperative setting of epilepsy: advanced imaging

Hans Urbach, DE

Imaging of pediatric epilepsy

Nadine Girard, FR

Interesting interactive epilepsy cases

Aikaterini Fitsiori , $\mathrm{CH}$

Coffee Break

ESNR meets Hungary Session 
$16: 30-16: 50$

Neuroradiology courses and meetings during the COVID-19 pandemic. An ESNR roadmap to continued education Gennaro D'Anna, IT

$16: 50-18: 00$ MS WK group news:

Jerome Hodel, FR

FRIDAY $1^{\text {st }}$ October 2021 - Meeting room 2

08:30 - 10:00

Moderators:

$08: 30-08: 50$
$08: 50-09: 10$
$09: 10-09: 30$
$09: 30-09: 50$

10:00-10:30

10:30-12:00

12:10 - 13:15

13:30-14:00

14:00 - 15:30

Moderator:

$14: 00-14: 20$

$14: 20-14: 40$

$14: 40-15: 00$

$15: 00-15: 30$

15:30 - 16:00

16:00 - 16:30

16:30 - 18:00

Moderators:

$16: 30-16: 50$

$16: 50-17: 10$

$17: 10-17: 30$

$17: 30-17: 50$
Spine NR: Pain: joint and nerves

Modern Radiology of the degenerative Spine

Johan van Goethem, $B E$

Nerve damage: radiological diagnosis

Matteo Bellini, IT

Percutaneous treatments - the joints: Injection, RF, Cryo

Adrian Kastler, FR

Percutaneous treatments - the nerves: peripheral nerve treatment

Allan Brook, US

Coffee Break

Opening Ceremony, Awards Ceremony, Honorary Members Ceremony (Meeting room 1)

Industry Sponsored Symposia

KEYNOTE LECTURE: Tele-expertise and tele-radiology in international activity,

A. Geissbuhler, $\mathrm{CH}$ (Meeting room 1)

INR: cAVMs

\author{
Arteriovenous malformations- Current status \\ Vitor Mendes Pereira, CA \\ Transvenous embolization of the pial AVMs \\ Charbel Mounayer, FR \\ Transarterial multiplug flow control technique for the curative treatment of pial AVMs \\ Saruhan Cekirge, TR \\ Embolization of dural AVFs: \\ Civan Islak, TR \\ Coffee Break \\ ESNR meets Hungary Session (meeting room 1) \\ Diag NR: Brain death and postmortem imaging
}

Principles of brain death and guidelines for postmortem neurological imaging

TBC

Post-mortem CT-A

$T B C$

Post-mortem pediatric and perinatal brain imaging

$T B C$

Pitfalls in brain death imaging

Sawicki $M, P L$

FRIDAY $1^{\text {st }}$ October 2021 - Meeting room 3
Interactive cases Spine NR

Selected oral presentations - Diagnostic

Coffee Break

Opening Ceremony, Awards Ceremony, Honorary Members Ceremony (Meeting room 1)

Industry Sponsored Symposia

KEYNOTE LECTURE: Tele-expertise and tele-radiology in international activity,

A. Geissbuhler, $\mathrm{CH}$ (Meeting room 1)

Selected Oral Presentations: Pediatric \& Head\&Neck

Coffee Break

ESNR meets Hungary Session (meeting room 1) 
16:30 - 18:00

INR: Innovations -INR

Moderator:
$16: 30-16: 50$
Future INR expectations
Paolo Machi, $\mathrm{CH}$
$16: 50-17: 10$
Robotic systems and intracranial aneurysms
Vitor Pereira, $C A$
$17: 15-18: 00$
Education panel:
Naci Kocer, Wim van Zwam, Jens Fiehler, Isll Saatci

FRIDAY $1^{\text {st }}$ October 2021 - Meeting room 4

$14: 00-15: 30$

Moderators:

$14: 00-14: 20$

$14: 20-14: 40$

$14: 40-15: 00$

$15: 00-15: 20$

15:30 - 16:00

16:00 - 16:30

16:30 - 18:00

\section{Spine NR: Present and the future}

Diagnostic and Technology Innovations (DTI, MRT and more)

Maria Isabel Vargas, $\mathrm{CH}$

High Field MRI on Spinal Cord

Majda Thurnher, AT

Dual Energy CT of the Spine

Roman Guggenberger, $\mathrm{CH}$

Advances in Spine Interventions

Luigi Manfrè, IT

Coffee Break

ESNR meets Hungary Session (meeting room 1)

Selected Oral Presentations: Interventional \& Spine

\section{SATURDAY $2^{\text {nd }}$ October 2021 - Meeting room 1}

08:00 - 08:30

08:30 - 10:00

Moderators:
$08: 30-08: 50$
08:50-09:10
09:10 - 09:30
09:30-09:50
10:00 - 10:30
10:30 - 12:00

\section{Moderator:}
$10: 30-10: 50$
$10: 50-11: 10$
$11: 10-11: 30$
$11: 30-11: 50$
$12: 10-13: 15$
13:30 - 14:00
14:00 - 15:30

Interactive cases Pediatric NR

Maria Argyropoulou, GR

ESNR - ESHNR joint session
Brachial plexus anatomy and pathology

Carlos Torres, CA

Extracranial neuro-vascular pathologies

Pedro Vilela, PT

Lymph nodes and lymphoma in the H\&N

Frank Pameijer, NL

Pre- and posttreatment imaging and staging of nasopharyngeal cancer -

Ann King, HK

Coffee Break

INR : Cerebral Aneurysms

The current status of EVT of intracranial ANs -what is the upper limit of indication?

Naci Kocer, TR

Bifurcation ANs - FD

Isil Saatci, TR

Balloon and/or stent assisted coiling in bifurcation

René Chapot, DE

Intrasaccular flow modifiers and flow disrupters in the EVT of bifurcation aneurysms

Laurent Spelle, FR

Industry Sponsored Symposia

KEYNOTE LECTURE: Spinal interventional neuroradiology

Isabel WANKE, CH

ASNR Joint session 
Diag NR: Intra- and extracranial vessel wall imaging

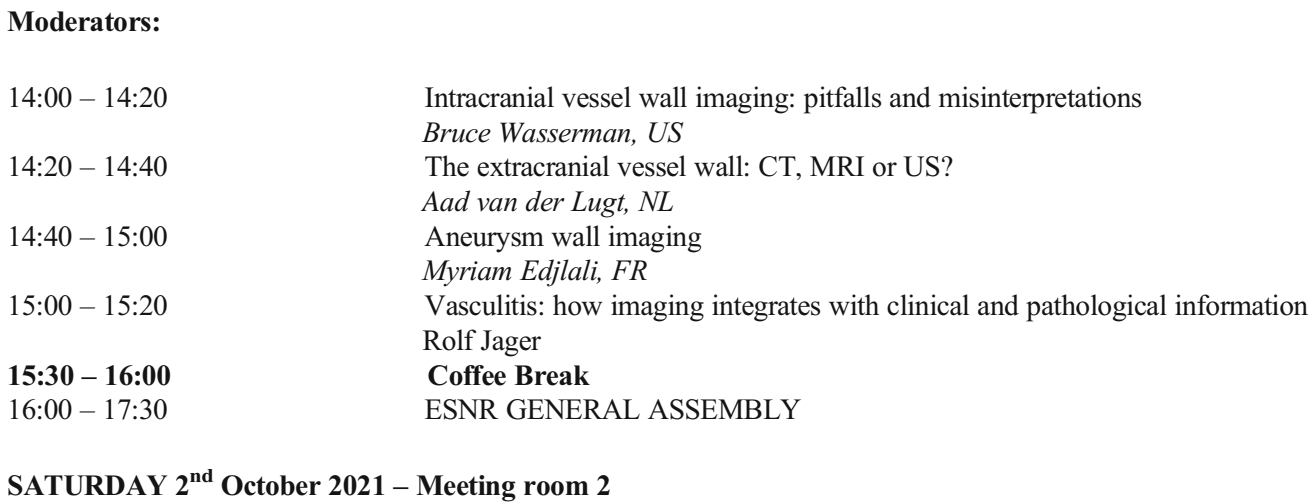

$08: 30-08: 50$

08:50 - 09:10

09:10 - 09:30

09:30 - 09:50

10:00 - 10:30

10:30 - 12:00

12:10 - 13:15

13:30 - 14:00

14:00 - 15:30

Moderator:

$14: 00-14: 20$
$14: 20-14: 40$
$14: 40-15: 00$
$15: 00-15: 20$
$15: 30-\mathbf{1 6 : 0 0}$
$16: 00-17 \cdot 30$

2nd trimester fetal MRI: through the ground glassAndrea Rossi, IT

3rd trimester MRI- can we help?

Chen Hoffmann, IL

Practical Approach to Fetal Ventriculomegaly -

Orit Glenn, US

MR Imaging Clues to Specific Diagnoses on Fetal MRI -

Usha Nagaraj, US

Coffee Break

ESNR GENERAL ASSEMBLY

SATURDAY $2^{\text {nd }}$ October 2021 - Meeting room 3

08:00 - 08:30
08:30 - 10:00

Moderator:

$08: 30-08: 50$

08:50 - 09:10

09:10-09:30
Interactive cases Head\&Neck NR Bernard Shuknecht, $\mathrm{CH}$

INR: INR varia
Endovascular treatment of Pediatric vascular malformations -

Guillaume Saliou, $\mathrm{CH}$

Subdural heamatoma

Markus Möhlenbruch, DE

Endovascular Therapy during COVID-19

A.Christine Januel, FR 

09:30-09:50
INR in pregnancy
10:00 - 10:30
Kamil Zelenak, SK
10:30 - 12:00
Coffee Break
ASSR Joint Session

Spine NR: The disc and biomechanics

\section{Moderators:}

$\begin{array}{ll}\text { 10:30 - 10:50 } & \text { The Disc \& Biomechanics: up to date on MR diagnosis } \\ & \text { TBC } \\ & \text { Adjacent level disease, biomechanics, imaging and intervention } \\ & \text { Bassem Georgy, US } \\ \text { 10:50 - 11:10 } & \text { Disc Diseases: How I destroy } \\ & \text { Joshua A Hirsch, US } \\ \text { 11:10 - 11:30 } & \text { Disc Diseases: How I Restore } \\ & \text { Alexis Kelekis, GR } \\ 11: 30-11: 50 & \text { Industry Sponsored Symposia } \\ & \text { KEYNOTE LECTURE: Spinal interventional neuroradiology } \\ \mathbf{1 2 : 1 0}-\mathbf{1 3 : 1 5} & \text { Isabel WANKE, } C H \\ \mathbf{1 3 : 3 0}-\mathbf{1 4 : 0 0} & \text { INR: i Stroke } \\ & \end{array}$

\section{Moderator:}

$\begin{array}{ll}\text { 14:00 - 14:20 } & \text { iStroke - New studies } \\ & \text { Wim van Zwam, NL } \\ & \text { First pass best results } \\ \text { 14:20 - 14:40 } & \text { Paolo Machi, CH } \\ & \text { Distal MT } \\ \text { 14:40 - 15:00 } & \text { Markus Möhlenbruch, DE } \\ & \text { Acute stroke treatment in the extended time window- } \\ 15: 00-15: 20 & \text { TBC } \\ & \text { Coffee Break } \\ \mathbf{1 5 : 3 0}-\mathbf{1 6 : 0 0} & \text { ESNR GENERAL ASSEMBLY }\end{array}$

SATURDAY $2^{\text {nd }}$ October 2021 - Meeting room 4

$14: 00-15: 30$

Spine NR: Tumors of Spine and Spinal Cord

Moderators:
$14: 00-14: 20$
Spine Tumors: What the Surgeon Wants To Know
$14: 20-14: 40$
Wende Gibbs, US
$14: 40-15: 00$
Up to date on Primary Tumors of the Spinal Cord Pia Sundgren, SE
Oncologic indications for Advanced Augmentation
$15: 00-15: 20$
Alexis Kelekis, GR
Update on Tumor embolization of the Spine \& Spinal Cord
Isabel Wanke, $\mathrm{CH}$

SUNDAY $3^{\text {rd }}$ October 2021 - Meeting room 1
08:00 - 08:30
Interactive cases NR
Clive Sperryn, SA
08:30 - 10:00
Diag NR: Advances in MRI: applications in clinical practice

How to make most of new possibilities of your MR system.

\section{Moderators:}

$08: 30-08: 50$

Synthetic MRI in Neuroradiology:

Ida Blystad, SE 
08:50-09:10

09:10 - 09:30

09:30 - 09:50

10:00 - 10:30

10:30 - 12:00

\section{Moderators:}

$10: 30-10: 50$

$10: 50-11: 10$

$11: 10-11: 30$

$11: 30-11: 50$

$12: 00-12: 30$
From SENSE to Compressed Sensing

Jan Casselman, BE

SWI: more than looking for microbleeds and calcifications:

Sven Haller, $\mathrm{CH}$

The promise and challenge of MR Spectroscopy for glioma molecular diagnosis.

Francesca Branzoli, FR

Coffee Break

INR: Future expectations in ICAs

Where do we stand in intracranial stenting: Which stent in which setting

Pedro Lylyk, AR

New evolution in stent design

Alessandra Biondi, FR

Surface modification

Zsolt Kulcsar, $\mathrm{CH}$

Antiplatelet therapy associated with the intracranial stent.

Paul Bhogal, UK

CLOSING CEREMONY

\section{SUNDAY $3^{\text {rd }}$ October 2021 - Meeting room 2}

08:00 - 08:30

08:30 - 10:00

Moderators:

$08: 30-08 \cdot 50$

08:50-09:10

09:10 - 09:30

09:30 - 09:50

10:00 - 10:30

10:30 - 12:00

\section{Moderators:}

$10: 30-10: 50$

$10: 50-11: 10$

$11: 10-11: 30$

$11: 30-11: 50$

$12: 00-12: 30$
Interactive cases INR

Kamil Zelenak

H\&N Anatomy and pathologies of the cranial nerves
Anatomy and pathologies of the Optic nerve

Sofie Van Cauter, BE

Anatomy and pathologies of the Oculomotor, trochlear and abducens nerves Carolina Tramontini, $\mathrm{CO}$

Anatomy and pathologies of the Trigeminal nerve

Katarina Surlan-Popovic, SI

Anatomy and pathologies of the Facial / vestibulo-cochlear nerves

Bernhard Schuknecht, $\mathrm{CH}$

Coffee Break

Diag NR: Quantitative imaging in neuroradiology practice

Quantitative imaging in MS

Frederik Barkhof, NL

Quantitative imaging in neurodegeneration

Meike Vernooij, $N L$

Quantitative imaging in neuro-oncology

Pia Sundgren, SE

Quantitative imaging in CSVD

Gennaro D'Anna, IT

CLOSING CEREMONY 
ORAL PRESENTATIONS

\section{DIAGNOSTIC - BRAIN}

1-O1

IS GADOLINIUM REALLY NEEDED FOR FOLLOW-UP MRI OF INTRACRANIAL MENINGIOMAS?

JOSE BOTO $^{1}$, Ramona Guatta ${ }^{2}$, Aikaterini Fitsiori ${ }^{1}$, Jeremy Hofmeister $^{3}$, Torstein R. Meling ${ }^{2}$, Maria Isabel Vargas Gomez ${ }^{1}$

${ }^{1}$ Division of Neuroradiology, Geneva University Hospital and Faculty of Medicine of Geneva, Rue Gabrielle-Perret-Gentil 4, 1205 Geneva, Switzerland, ${ }^{2}$ Division of Neurosurgery, Lugano Regional Hospital (Civic), Via Tesserete 46, 6900 Lugano, Switzerland, ${ }^{3}$ Division of Radiology, Geneva University Hospital and Faculty of Medicine of Geneva, Rue Gabrielle-Perret-Gentil 4, 1205 Geneva, Switzerland

Keywords: Meningioma, MRI, Gadolinium

Introduction: Notwithstanding the well-established safety of Gd compounds, repeat administration of Gd-based contrast may lead to tissue deposition of gadolinium, which has given rise to recent concerns. In view of this, new trends in performing non-contrast MRI whenever possible are being championed. Taking these points into consideration, the purpose of our study was to assess the value of Gd-based contrast media for routine follow-up MRIs of intracranial meningiomas in so far as tumor size and growth are concerned.

Material \& Methods: Our institutional review board approved this retrospective cohort study. Patient consent was waived. All patients with meningiomas who underwent brain MRI between 2010 and 2015 in our institution were retrieved and a total of 123 patients ( 36 males, $87 \mathrm{fe}-$ males; mean age 64.5 years; range 22.8-96.0 years) were included, producing a total number of 282 MRI scans. All meningiomas were segmented using a semi-automated software (IntelliSpace from Philips) on T1-3D Gd and axial T2WI. Shape and volume features were then automatically computed for all meningiomas using Pyradiomics (version 2.2.0). Agreement between T1-3D Gd and T2WI was assessed by the intraclass correlation coefficient.

Results: There was excellent agreement between T1-3D Gd and T2WI derived parameters $(\mathrm{p}<0.001)$ : tumor volume $(\mathrm{ICC}=0.996)$, surface area (ICC=0.989), surface/volume ratio ( $\mathrm{ICC}=0.924)$, maximum 3D diameter (ICC $=0.986$ ), maximum 2D diameter in the axial $(\mathrm{ICC}=0.990)$, coronal (ICC $=0.982)$, and sagittal planes $(\mathrm{ICC}=0.984)$, major axis length ( $\mathrm{ICC}=0.989)$, minor axis length $(\mathrm{ICC}=0.992)$, least axis length $(\mathrm{ICC}=0.988$ ). Annual tumor growth also showed good agreement between the two sequences $(\mathrm{p}<0.001)$.

Discussion: The absolute agreement between parameters obtained from the T1 3D Gd and T2WI sequences were near perfect, despite the fact that T2WI sequences were 2D with $4 \mathrm{~mm}$ slice thickness and a $0.4 \mathrm{~mm}$ gap between slices, demonstrating the value of such non-contrast sequences for the follow-up of untreated meningiomas. This proved to be case across a variety of scanner models from three different manufacturers, for meningiomas in different locations, in smaller versus larger meningiomas, and also symptomatic versus asymptomatic lesions, thus reinforcing the robustness of the agreement we found between T1 3D Gd and T2WI segmentations.

Conclusion: Our results show excellent agreement between size and growth of meningiomas derived from T1 3D Gd and T2WI, questioning the added value of gadolinium-based contrast in follow-up MRI of meningiomas. Furthermore, a non-contrast MRI would avert risks associated with contrast media and be more cost effective.

Table 1 Agreement between dimension and geometric parameters of 132 meningiomas ( 61 of which at different time points) from 122 patients derived from semi-automated segmentation performed on the T1 3D Gd and axial T2 sequences

\begin{tabular}{|c|c|c|c|c|c|}
\hline & $\mathrm{N}$ & $\begin{array}{l}\text { T1 3D Gd } \\
(\text { mean } \pm \text { SD) }\end{array}$ & $\begin{array}{l}\text { Axial T2 } \\
(\text { mean } \pm \mathrm{SD})\end{array}$ & ICC & $\mathrm{p}$ \\
\hline Tumor volume $\left(\mathrm{mm}^{3}\right)$ & 281 & $9,012.15 \pm 119,223.03$ & $8,528.45 \pm 18,368.18$ & 0.996 & $<0.001$ \\
\hline Tumor surface area $\left(\mathrm{mm}^{2}\right)$ & 281 & $2,010.39 \pm 2,798.64$ & $2,243.01 \pm 3,236.91$ & 0.989 & $<0.001$ \\
\hline Surface/volume ratio $\left(\mathrm{mm}^{-1}\right)$ & 281 & $0.56 \pm 0.29$ & $0.67 \pm 0.38$ & 0.924 & $<0.001$ \\
\hline Maximum 3D diameter (mm) & 281 & $26.21 \pm 15.76$ & $27.49 \pm 16.02$ & 0.986 & $<0.001$ \\
\hline Maximum 2D diameter in the axial plane (mm) & 281 & $24.13 \pm 14.85$ & $22.69 \pm 14.85$ & 0.990 & $<0.001$ \\
\hline Maximum 2D diameter in the coronal plane ( $\mathrm{mm})$ & 281 & $23.15 \pm 13.79$ & $24.84 \pm 14.75$ & 0.982 & $<0.001$ \\
\hline Maximum 2D diameter in the sagittal plane (mm) & 281 & $23.99 \pm 14.95$ & $25.35 \pm 15.29$ & 0.985 & $<0.001$ \\
\hline Major axis length $(\mathrm{mm})$ & 281 & $21.92 \pm 13.16$ & $22.48 \pm 13.00$ & 0.989 & $<0.001$ \\
\hline Minor axis length (mm) & 281 & $18.04 \pm 11.19$ & $17.84 \pm 11.47$ & 0.992 & $<0.001$ \\
\hline Least axis length (mm) & 281 & $13.83 \pm 9.02$ & $13.08 \pm 9.32$ & 0.988 & $<0.001$ \\
\hline
\end{tabular}

$\mathrm{N}=$ number of measurements; $\mathrm{SD}=$ standard deviation; $\mathrm{ICC}=$ intraclass correlation coefficient 
Table 2 Agreement between tumor growth based on dimension and geometric parameters of 30 meningiomas from 26 patients derived from semiautomated segmentation performed on the T1 3D Gd and axial T2 sequences (values expressed as absolute change and percent change per year)

\begin{tabular}{|c|c|c|c|c|c|c|}
\hline & $\mathrm{N}$ & Unit & $\begin{array}{c}\mathrm{T} 1 \text { 3D Gd } \\
(\text { mean } \pm \mathrm{SD})\end{array}$ & $\begin{array}{c}\text { Axial T2 } \\
(\text { mean } \pm \mathrm{SD})\end{array}$ & $\mathrm{ICC}$ & $\mathrm{p}$ \\
\hline Tumor volume & 51 & $\begin{array}{c}\mathrm{mm}^{3} / \text { year } \\
\% / \text { year }\end{array}$ & $\begin{aligned} 461.87 & \pm 2704.17 \\
9.24 & \pm 22.55\end{aligned}$ & $\begin{array}{c}556.64 \pm 2624.02 \\
15.82 \pm 32.00\end{array}$ & $\begin{array}{l}0.989 \\
0.868\end{array}$ & $\begin{array}{l}<0.001 \\
<0.001\end{array}$ \\
\hline Tumor surface area & 51 & $\begin{array}{c}\mathrm{mm}^{2} / \text { year } \\
\% / \text { year }\end{array}$ & $\begin{aligned} 87.03 & \pm 371.66 \\
5.73 & \pm 12.66\end{aligned}$ & $\begin{array}{c}112.01 \pm 453.59 \\
8.24 \pm 16.81\end{array}$ & $\begin{array}{l}0.973 \\
0.889\end{array}$ & $\begin{array}{l}<0.001 \\
<0.001\end{array}$ \\
\hline Surface/volume ratio & 51 & $\begin{array}{r}\mathrm{mm}^{-1} / \\
\text { year }\end{array}$ & $\begin{array}{l}-0.01 \pm 0.03 \\
-0.91 \pm 4.34\end{array}$ & $\begin{array}{l}-0.02 \pm 0.06 \\
-2.35 \pm 5.74\end{array}$ & $\begin{array}{l}0.771 \\
0.672\end{array}$ & $\begin{array}{l}<0.001 \\
<0.001\end{array}$ \\
\hline Maximum 3D diameter & 51 & $\begin{array}{c}\text { \%/year } \\
\mathrm{mm} / \text { year } \\
\% / \text { year }\end{array}$ & $\begin{array}{l}0.56 \pm 1.79 \\
2.38 \pm 6.12\end{array}$ & $\begin{array}{l}0.72 \pm 1.95 \\
3.16 \pm 7.02\end{array}$ & $\begin{array}{l}0.922 \\
0.849\end{array}$ & $\begin{array}{l}<0.001 \\
<0.001\end{array}$ \\
\hline Maximum 2D diameter in the axial plane & 51 & $\begin{array}{c}\mathrm{mm} / \text { year } \\
\% / \text { year }\end{array}$ & $\begin{array}{l}0.44 \pm 1.22 \\
2.29 \pm 5.20\end{array}$ & $\begin{array}{l}0.49 \pm 1.88 \\
2.44 \pm 6.69\end{array}$ & $\begin{array}{l}0.836 \\
0.683\end{array}$ & $\begin{array}{l}<0.001 \\
<0.001\end{array}$ \\
\hline Maximum 2D diameter in the coronal plane & 51 & $\begin{array}{c}\mathrm{mm} / \text { year } \\
\% / \text { year }\end{array}$ & $\begin{array}{l}0.35 \pm 1.13 \\
1.82 \pm 4.55\end{array}$ & $\begin{array}{l}0.54 \pm 1.60 \\
3.16 \pm 8.97\end{array}$ & $\begin{array}{l}0.755 \\
0.631\end{array}$ & $\begin{array}{l}<0.001 \\
<0.001\end{array}$ \\
\hline Maximum 2D diameter in the sagittal plane & 51 & $\begin{array}{l}\mathrm{mm} / \text { year } \\
\% / \text { year }\end{array}$ & $\begin{array}{l}0.53 \pm 1.74 \\
2.46 \pm 6.25\end{array}$ & $\begin{array}{l}0.81 \pm 1.73 \\
4.12 \pm 7.31\end{array}$ & $\begin{array}{l}0.901 \\
0.752\end{array}$ & $\begin{array}{l}<0.001 \\
<0.001\end{array}$ \\
\hline Major axis length & 51 & $\begin{array}{l}\mathrm{mm} / \text { year } \\
\% / \text { year }\end{array}$ & $\begin{array}{l}0.51 \pm 1.57 \\
2.42 \pm 5.86\end{array}$ & $\begin{array}{l}0.67 \pm 1.64 \\
3.52 \pm 7.45\end{array}$ & $\begin{array}{l}0.930 \\
0.820\end{array}$ & $\begin{array}{l}<0.001 \\
<0.001\end{array}$ \\
\hline Minor axis length & 51 & $\begin{array}{l}\mathrm{mm} / \text { year } \\
\% / \text { year }\end{array}$ & $\begin{array}{l}0.28 \pm 0.80 \\
2.10 \pm 4.49\end{array}$ & $\begin{array}{l}0.36 \pm 0.96 \\
3.11 \pm 7.05\end{array}$ & $\begin{array}{l}0.777 \\
0.702\end{array}$ & $\begin{array}{l}<0.001 \\
<0.001\end{array}$ \\
\hline Least axis length & 49 & $\begin{array}{c}\mathrm{mm} / \text { year } \\
\% / \text { year }\end{array}$ & $\begin{array}{l}0.20 \pm 0.83 \\
1.90 \pm 5.38\end{array}$ & $\begin{array}{l}0.31 \pm 1.09 \\
2.89 \pm 7.86\end{array}$ & $\begin{array}{l}0.838 \\
0.753\end{array}$ & $\begin{array}{l}<0.001 \\
<0.001\end{array}$ \\
\hline
\end{tabular}

$\mathrm{N}=$ number of measurements; $\mathrm{SD}=$ standard deviation; $\mathrm{ICC}=$ intraclass correlation coefficient

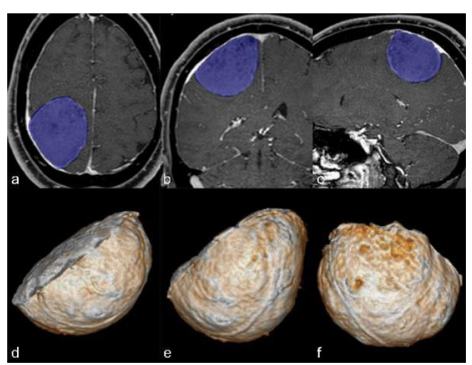

Figure 1 Forty-one year-old man. Meningioma of the right convexity adjacent to the parietal lobe. Segmentation of the meningioma is achieved by shading the lesion in blue using in a semi-automated software $(\mathrm{a}, \mathrm{b}, \mathrm{c})$. The voxels outside the shaded volume can be discarded and the voxels within the volume (shown as 3D rendering in $\mathrm{d}$, $\mathrm{e}$ and $\mathrm{f}$ ) any other software for further analysis.

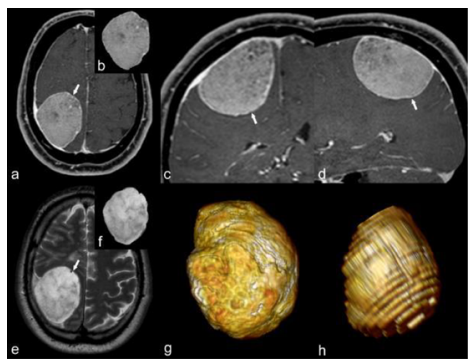

Figure 2 Same patient as in Figure Meningioma of the right convexity adjacent to the parietal lobe. Axial (a), coronal (c) and sagittal (d) THRIVE Gd and axial T2W (e) images depict a meningioma of the right convexity exerting mass effect on the adjacent brain parenchyma of the parietal lobe (arrow in a, c, $d$ and e). The insets show axial THRIVE Gd (b) and T2W (f) images of the isolated lesion obtained with the semi-automated software, from which $3 \mathrm{D}$ rendering can easily be performed with any DICOM viewer ( $\mathrm{g}$

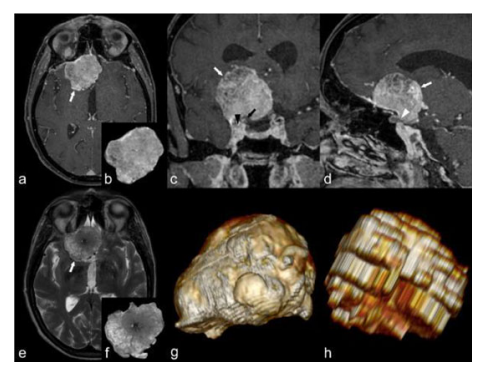

Figure 3 Fifty-two year-old woman. Axial (a), coronal (c) and sagittal (d) THRIVE Gd and axial T2W (e) images show a meningioma arising from the planum sphenoidale and orbital roof on the right (white arrow in a, c, d and e). The lesion crosses the midline and is in close contact with the cisternal segment of the right optic nerve (white arrowhead in d), the supra-clinoid right internal carotid artery the (he cavenous sinus (black arrowhead in c). T2W (f) images of the isolated lesion obtained

with the semi-automated software, from which 3D rendering was performed with our DICOM viewer ( $\mathrm{g}$ T1 3D FS Gd, h T2WI). This case illustrates a particularly challenging meningioma for which the skull base location, close contact with other structures and irregular shape of the lesion made the segmentation process more
difficult.

\section{1-O2}

TECHNICAL AND CLINICAL VALIDATION OF COMMERCIAL AUTOMATED VOLUMETRIC MRI TOOLS FOR DEMENTIA DIAGNOSIS - A SYSTEMATIC REVIEW

HUGH G PEMBERTON ${ }^{1,2,3}$, Lara A M Zaki ${ }^{4}$, Olivia Goodkin ${ }^{1,2}$, Ravi $\mathrm{K}$ Das $^{5}$, Sjoerd B Vos ${ }^{1,2}$, Rebecca M E Steketee ${ }^{4}$, Frederik Barkhof ${ }^{1,2,6}$, Meike W Vernooij ${ }^{4,7}$

${ }^{1}$ Centre for Medical Image Computing (CMIC), Department of Medical Physics and Bioengineering, University College London, London, United Kingdom. ${ }^{2}$ UCL Queen Square Institute of Neurology, University College London, London, United Kingdom, ${ }^{3}$ Dementia 
Research Centre, UCL Queen Square Institute of Neurology, University College London, London, United Kingdom, ${ }^{4}$ Department of Radiology and Nuclear Medicine, Erasmus MC University Medical Center, Rotterdam, the Netherlands, ${ }^{5}$ Clinical, Educational and Health Psychology, University College London, London, United Kingdom, ${ }^{6}$ Radiology \& Nuclear Medicine, VU University Medical Center, Amsterdam, the Netherlands, ${ }^{7}$ Department of Epidemiology, Erasmus MC University Medical Center Rotterdam, the Netherlands

Keywords: AI, quantitative MRI, volumetry, dementia, atrophy

Developments in neuroradiological MRI analysis offer promise in enhancing objectivity and consistency in dementia diagnosis through the use of quantitative volumetric reporting tools (QReports). However, published validation of commercial/proprietary tools is limited and pathways for clinical integration are varied. To this end, the Quantitative Neuroradiology Initiative (QNI) was developed previously as a framework for embedding QReports into clinical neuroradiology workflows and comprises the following steps:

(1) establishing an area of clinical need and identifying appropriate proven imaging biomarker(s)

(2) developing a method for automated analysis of these biomarkers, by designing an algorithm and compiling reference data

(3) communicating the results via an intuitive and accessible QReport

(4) technically and clinically validating the proposed tool pre-use

(5) integrating the developed analysis pipeline into the clinical reporting workflow

(6) performing in-use evaluation

In the current project, we systematically review published evidence (Figure 1) regarding 17 QReports found via FDA database and conference searching, and to what extent this evidence fulfils steps 4-6 of the QNI framework. We also summarise unbiased technical details of available products in order to increase transparency of evidence and present the range of reporting tools on the market (Table 1). Our intention is to assist neuroradiologists in making informed decisions regarding adoption of these methods in the clinic. Study categories and inclusion criteria were:

Technical validation - For example, test-retest studies, standalone receiver operating characteristics or comparing volumetric results to manual segmentation and/or state-of-the-art segmentation software.

Clinical validation - Papers testing QReport use by clinician end-users on a dementia/memory clinic population aiming to assess the QReport's effect and impact on clinical management.

Workflow integration and in-use evaluation - Papers analysing benefit to patients, effect on reporting time, clinical and population perception or socioeconomic effects of using QReports.

A total of 62 original studies covering technical (39) or clinical validation (23, dementia $=15$, other neurological diseases $=8)$ were identified from 11 of the 17 companies/products assessed. For 6 products, no publications meeting our inclusion criteria were identified. Only 4 vendors have published clinical validation of their reports in a dementia population (Figure 2). No evidence of QReport in-use evaluation was found.

We conclude that there is a significant evidence gap regarding clinical validation and in-use evaluation of these tools in dementia MRI diagnosis. We hope this work encourages such validation studies from the developers of these quantitative tools and recommend caution from clinicians when examining claims of the tools' clinical performance.
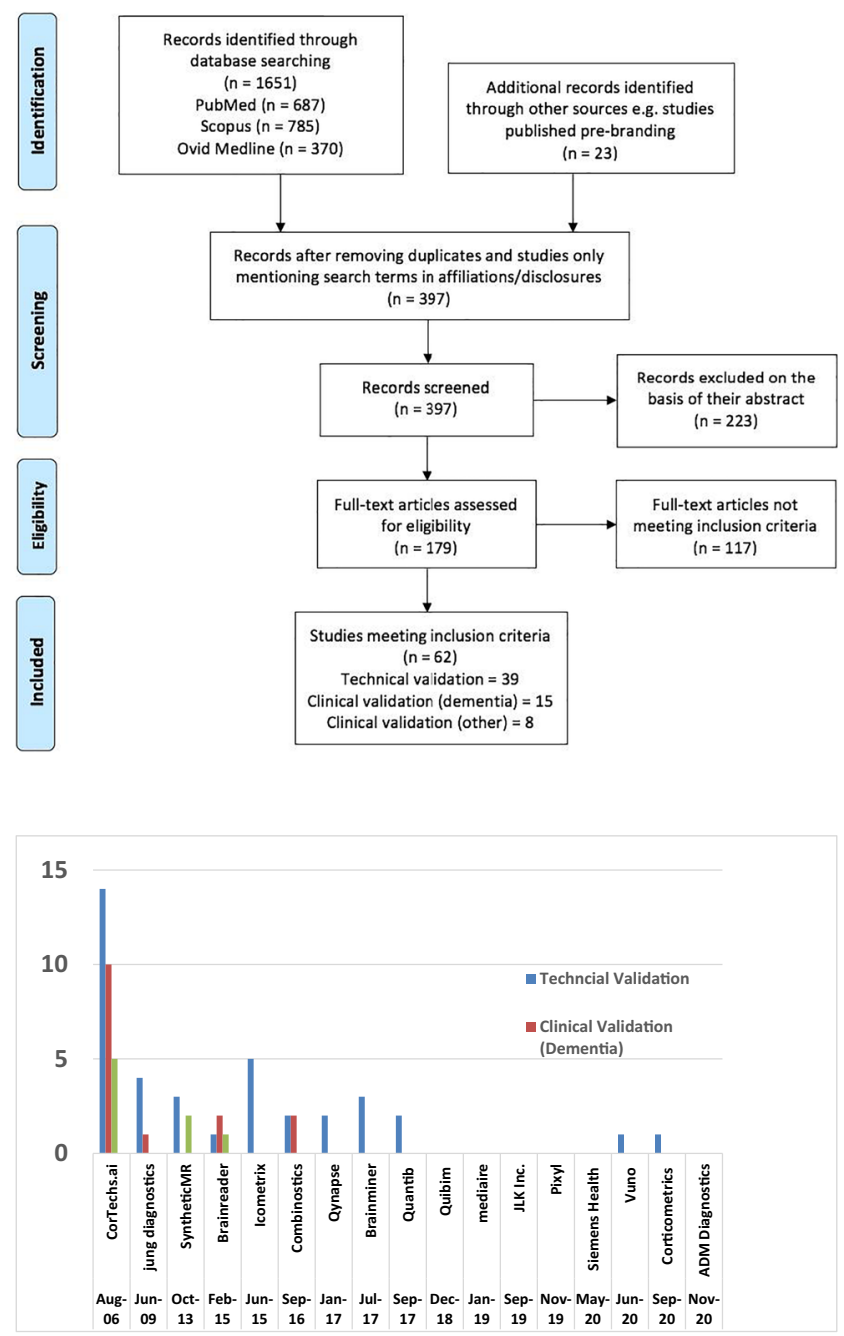

Figure 2 The distribution of papers meeting our inclusion criteria for each of the companies identified. The vendors are listed in chronological order according to the date of their first $\mathrm{CE} / \mathrm{FDA}$ approval

Table 1 A high-level database of the vendors and various features in each of their QReports, presented in alphabetical order of vendor name. We have outlined information from publications and direct contact with vendors for readers to assess according to their individual needs. All information was checked and confirmed with vendors in advance of publication. Differing amounts of information between vendors is due to variation in how much the vendors were willing/able to share. Due to the proprietary nature of reports, it was not possible to independently verify all details from vendors but they were confirmed against sample reports where possible. Abbreviations: Convolutional Neural Network (CNN); Voxel-Based Morphometry (VBM); Statistical Parametric Mapping (SPM); Geodesic Information Flow (GIF); Traumatic Brain Injury (TBI); Virtual Machine (VM); General Electric (GE); White Matter Hyperintensity (WMH); Signal to Noise Ratio (SNR); Contrast to Noise Ratio (CNR); Quality Control (QC); Intracranial Volume (ICV); picture archiving and communication system (PACS) 


\begin{tabular}{|c|c|c|c|c|c|c|c|c|c|c|c|c|c|}
\hline vendor & $\begin{array}{l}\text { Protatur } \\
\text { name }\end{array}$ & 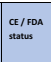 & 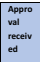 & 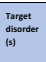 & 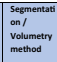 & 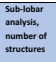 & 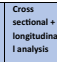 & 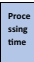 & 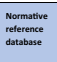 & 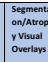 & 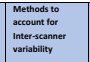 & 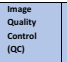 & $\begin{array}{l}\text { oeplomenen } \\
t^{2}\end{array}$ \\
\hline 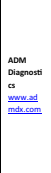 & $\begin{array}{c}\text { Contian } \\
\text { sement }\end{array}$ & 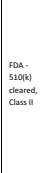 & 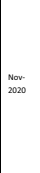 & Dementia & 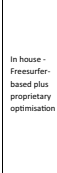 & 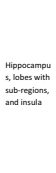 & 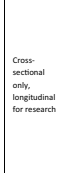 & 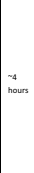 & 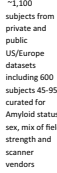 & 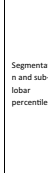 & 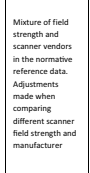 & 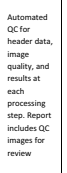 & mperesess \\
\hline 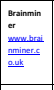 & onberm & $c_{1}^{-c \cos s}$ & $\begin{array}{l}\text { sum } \\
\text { col7 }\end{array}$ & Dementia & Gefsat & ves, 30 & 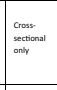 & 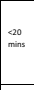 & 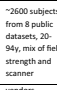 & 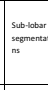 & 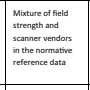 & 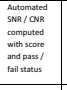 & 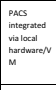 \\
\hline 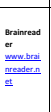 & \begin{tabular}{|l} 
Nevero \\
rester
\end{tabular} & 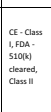 & 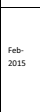 & 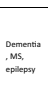 & 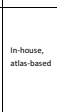 & ves,4s & 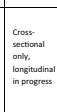 & 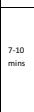 & 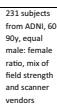 & 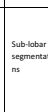 & 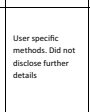 & 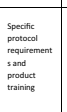 & 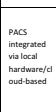 \\
\hline 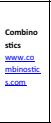 & 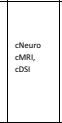 & 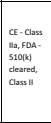 & 20 & $\begin{array}{l}\text { exementia } \\
\text { ens }\end{array}$ & 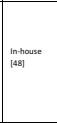 & versiar. & 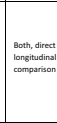 & $\begin{array}{l}-15 \\
\text { mins } \\
\text { ming }\end{array}$ & 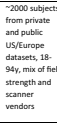 & 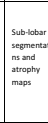 & 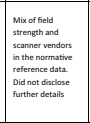 & 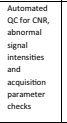 & 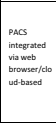 \\
\hline 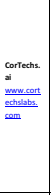 & Neverou & 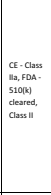 & 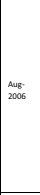 & 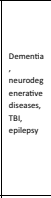 & 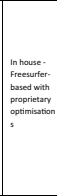 & $\operatorname{ves}, 75+$ & 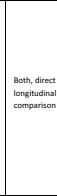 & 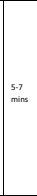 & 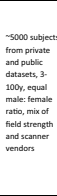 & 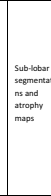 & 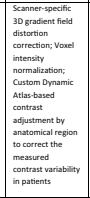 & 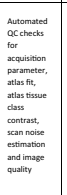 & 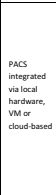 \\
\hline 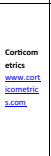 & ruma & 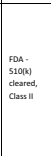 & 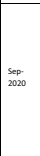 & 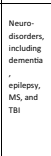 & 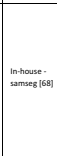 & 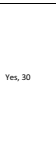 & 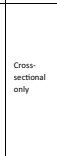 & 1 hour & 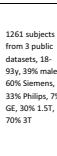 & 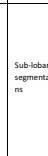 & 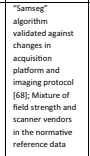 & 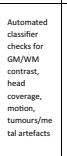 & 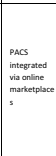 \\
\hline 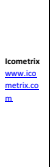 & $\begin{array}{l}\text { ictabian } \\
\text { atam }\end{array}$ & 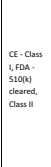 & $\begin{array}{l}\text { walt } \\
\text { 2015 }\end{array}$ & Dementia & Intowese & 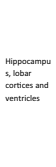 & 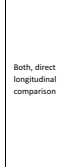 & 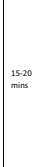 & 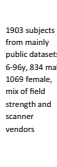 & 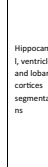 & 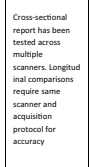 & 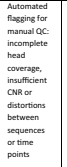 & 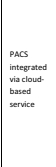 \\
\hline 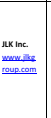 & 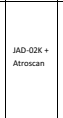 & $a_{1}^{a-\operatorname{coses}}$ & $\begin{array}{c}\text { sep } \\
\text { cong }\end{array}$ & oementia & 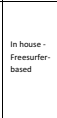 & $v_{e s, 62}$ & Both & 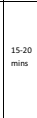 & 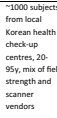 & 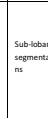 & 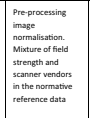 & 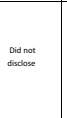 & 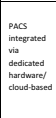 \\
\hline 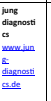 & tionetic & $\alpha_{1} \cdot \operatorname{coses} s$ & 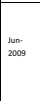 & 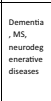 & 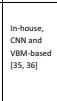 & 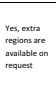 & 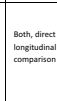 & 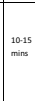 & 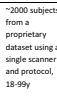 & 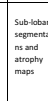 & 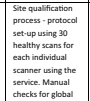 & 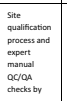 & 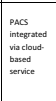 \\
\hline
\end{tabular}

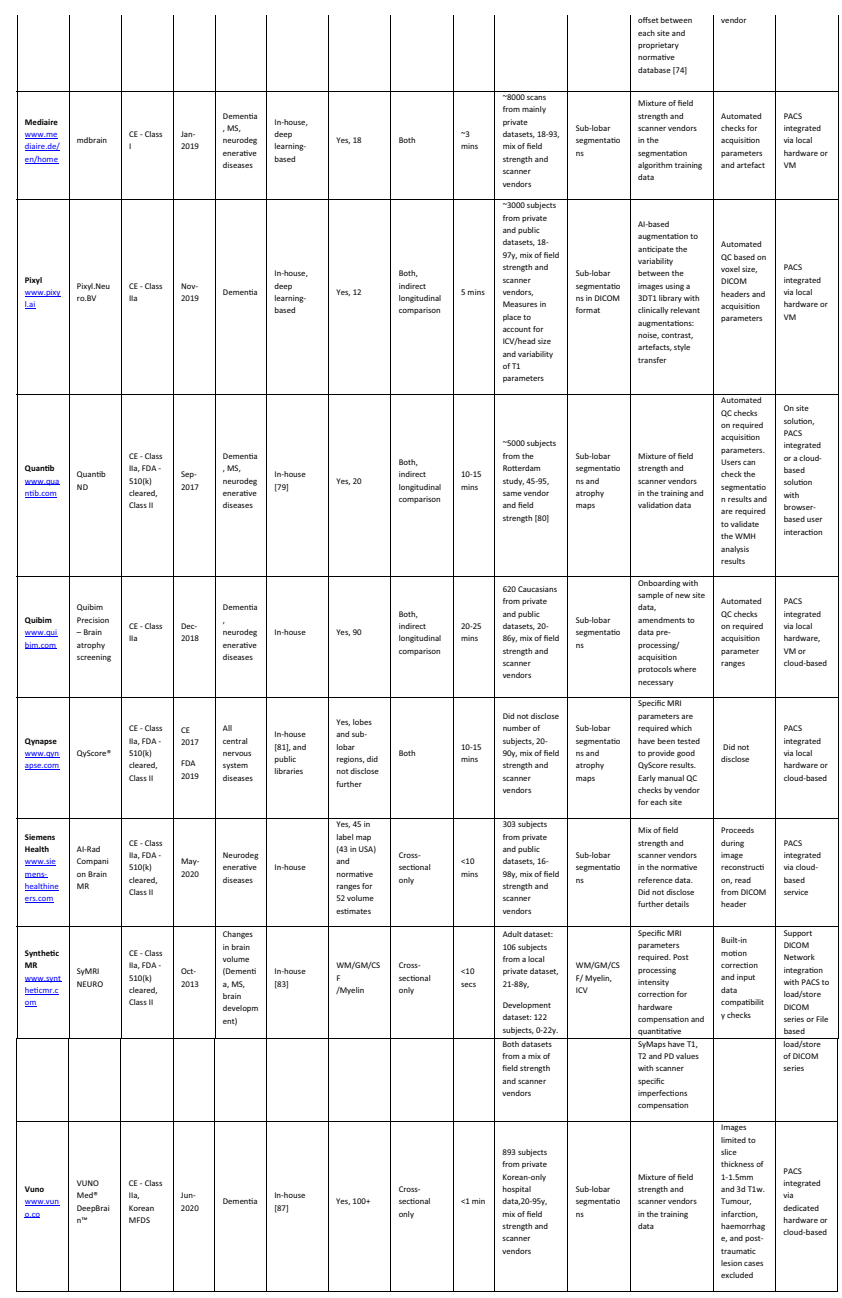

\section{1-O3}

PREDICTION OF MENINGIOMA CONSISTENCY WITH QUANTITATIVE MRI

$\underline{\text { BENEDICTE M. A. DELATTRE }}^{1}$, Torstein R. Meling ${ }^{2}$, Maria Isabel Vargas Gomez ${ }^{3}$

${ }^{1}$ Division of radiology, Geneva University Hospital, Geneva, Switzerland, ${ }^{2}$ Division of neurosurgery, Geneva University Hospital, Geneva, Switzerland, ${ }^{3}$ Division of neuroradiology, Geneva University Hospital, Geneva, Switzerland

Keywords: quantitative MRI, synthetic MR, meningioma

Introduction: Prediction of meningioma consistency prior to surgery is very important for the planning of the intervention, as well as for evaluation of the risks associated with the surgery. Indeed, a firm tumor requires more physical energy from the Cavitron Ultra-Sonic Aspirator (CUSA) to remove the tumor, energy that is transmitted to the perilesional structures such as brain tissue, cranial nerves, cortical arteries and veins. Hence, a firm meningioma is associated with higher risk of neurological complications and bleeding. MRI has already shown to have the potential to predict meningioma consistency and in this study, we hypothesize that quantitative MRI parameters may accurately help predict meningioma consistency. 
Methods: Presurgical MRI was performed at $3 \mathrm{~T}$ including the acquisition of diffusion-weighted imaging and syntheticMR sequence allowing the generation of quantitative T1, T2 and PD maps (using SyMR software, Linköping, Sweden), acquisition time $6 \mathrm{~min} 30 \mathrm{sec}$. Eighteen patients were included in the study (10 female, 8 male; mean age 58 years). T1 (in ms), $\mathrm{T} 2$ (in $\mathrm{ms}$ ), $\mathrm{PD}$ (in \%) and $\mathrm{ADC}$ (in $\mathrm{mm}^{2} / \mathrm{s}$ ) were measured in the meningioma and data were collected blinded to the consistency. Meningioma consistency was objectively graded during surgery using CUSA score from 1 (extremely soft) to 5 (extremely firm).

Results: Despite the fact that there was no statistical difference between quantitative MRI parameters and CUSA scale (Figure 1), we observed a linear correlation between $\mathrm{T} 1$ and $\mathrm{T} 2$ relaxation time and CUSA scale ( $R=0.52$ and $R=0.43$, respectively). We can also see that lesion appears more hyperintense on $\mathrm{T} 1$ and $\mathrm{T} 2$ for soft meningioma than for firm one (Figure 2). However, it was less obvious for PD and $\mathrm{ADC}(\mathrm{R}=0.14$ and $\mathrm{R}=0.35$, respectively)

Discussion \& Conclusion: This study shows that consistency could be predicted by quantitative MRI parameters, in particular T1 and T2 (in ms) derived with SyntheticMR sequence. This sequence allows also obtaining conventional contrasts for the same acquisition time (T1-W, T2-W, PDW) and could therefore be used without impacting the total acquisition time of the protocol. We were unable to show a statistical difference between quantitative parameters and consistency due to the limited number of cases with very soft (CUSA $=1$ or 2 ) or very firm $(C U S A=5)$ tumor consistency. These results should therefore be confirmed by increasing patient inclusion. However, using quantitative parameters is a promising technique which does not depend on subjective measurement of the signal on conventional MRI images.

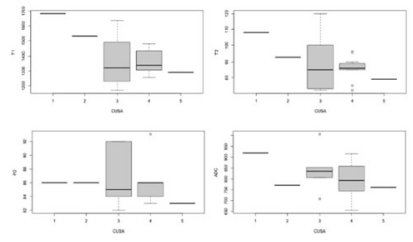

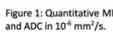

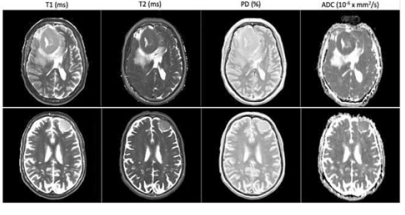

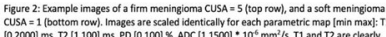

1-O4

COMPARISON BETWEEN CONTRAST ENHANCEMENT MPRAGE AND CS-VIBE ACCELERATES MRI TECHNIQUES FOR DIAGNOSIS OF PATIENTS AT RISK OF INTRACRANIAL METASTASIS

\section{YOUNGHEE YIM $^{1}$, Mi Sun Chung ${ }^{1}$, Jun Soo Byun ${ }^{1}$}

${ }^{1}$ Department of Radiology, Chung-Ang University, College of Medicine, Chung-Ang University Hospital, Seoul, Republic of Korea

Keywords: MRI, metastasis, brain, enhancement

Objective: We aimed to compare the diagnostic performance of postcontrast 3D compressed-sensing volume-interpolated breath-hold examination (CS-VIBE) and 3D T1 magnetization-prepared rapid-acquisition gradient-echo (MPRAGE) in detecting metastasis. We also examined and compared image quality between both sequences.

Materials \& Methods: Between July 2020 and December 2020, 120 patients who underwent contrast-enhanced brain MRI to evaluate metastasis with both conventional MPRAGE and CS-VIBE (scan time: 5 minutes 48 seconds vs. 2 minutes 44 seconds) were included in this retrospective study. All images were independently reviewed by two radiologists for the presence of enhancing metastatic lesions. Signal-to-noise ratio (SNR) of the pons and both centrum semiovale were compared between two sequences. In patients with intracranial metastasis, enhancement degree and contrastto-noise ratio (CNR parenchyma-enhancing lesion) of the lesion were measured. The overall SNR, image quality, white-gray matter discrimination and enhancing lesion conspicuity were analyzed.

Result: In qualitative analysis, SNR and enhancing lesion conspicuity showed moderate to excellent agreement between MPRAGE and CSVIBE $(\mathrm{k}=0.73-0.97)$ in two observations while overall image quality and gray-white matter differentiation showed moderate to good agreement $(\mathrm{k}=0.51-0.79)$. Both MPRAGE and CS-VIBE equally detected enhancing intracranial lesions. Total 22 patients had enhancing intracranial metastasis, and CNR (20.2 \pm 11.8 vs. $20.7 \pm 16.5)$ and contrast rate of MPRAGE (57.7 \pm 47.6 vs. $59.1 \pm 53.2)$ showed lower than that of CSVIBE. Overall, MPRAGE showed more best image quality than that of CS-VIBE (30.4\% vs. $26.7 \%$ ), but CS-VIBE showed less blurred images than that of MPRAGE (15.0\% vs. 20.4\%). When comparing the preference, MPRAGE was preferred in $11.3 \%$ (27 out of 240 observations) while CS-VIBE was preferred in $15.4 \%$ (37 out of 240 observations).

Conclusion: CS-VIBE postcontrast technique achieved comparable image quality and visualization compared to the conventional MPRAGE, with the scan time being half of that of MPRAGE.

\section{1-05}

VESSEL WALL MR IMAGING IN CEREBROVASCULAR DISEASES

ANAIT K. NIKOGOSOVA ${ }^{1}$, Tatiana Rostovtseva ${ }^{1}$, Anastasia Vishnyakova $^{1}$, Alexander Berdalin ${ }^{1}$, Gubskiy Ilya ${ }^{1}$, Vladimir G. Lelyuk ${ }^{1}$

${ }^{1}$ FSBI "Federal Center for Brain and Neurotechnologies" of the Federal Medical and Biological Agency, Moscow, Russian Federation

Keywords: vessel wall imaging, cerebrovascular diseases, stroke, MRI

Introduction: Vascular wall imaging (VWI) is a relatively new MRI technique that is increasingly used as a non-invasive diagnostic tool for the evaluation of intra- and extracranial cerebral arteries. Data obtained via VWI provides new insight into the pathogenesis of the brachiocephalic arteries atherosclerosis and allows to develop new effective methods for early diagnosis and treatment of the brain vascular diseases. The purpose of this study was to describe VWI-patterns of vessel wall changes in patients with cerebrovascular diseases.

Methods: A total of 64 ischemic stroke patients within one year from onset were recruited to this study. Magnetic resonance angiography and $\mathrm{CT}$ angiography were performed for the assessment of intracranial arterial stenoses. Further high-field MR unit (3 Tesla MRI Scanner) was used to obtain vessel wall MR sequences (high resolution pre- and post contrast T1-weighted images).

Results: Weak circumferential enhancement without thickening of the carotid bulb vascular wall was observed in all patients without stenotic lesions. Moreover, $91 \%$ of examined patients without stenotic lesions showed circumferential enhancement without arterial wall thickening of V4 segments of vertebral arteries; the severity and length of this changes correlated with age $(\mathrm{p}<0.01)$. In patients with stenotic atherosclerotic lesions eccentric thickening of the wall with contrast enhancement was observed. In all patients with chronic thrombotic occlusions a vivid circumferential enhancement in combination with a decrease of lumen diameter was found. Location of vessel wall lesions correlated with location of the brain infarcts $(\mathrm{p}<0.01)$. 
Discussion \& Conclusion: Intracranial vascular diseases are usually investigated via lumen-based techniques such as magnetic resonance angiography, computed tomography angiography and digital subtraction angiography. Meanwhile the assessment of non-stenotic vascular lesions using these methods is not possible. High-resolution magnetic resonance VWI complements traditional luminal studies in the evaluation of cerebrovascular disease by visualizing changes in the vascular wall. However further researches are needed to determine clinical significance of VWI findings and to correlate this findigns with histopathology.

\section{1-O6}

DIVERGENT MAGNETIC RESONANCE IMAGING ATROPHY PATTERNS IN ALZHEIMER'S DISEASE AND PRIMARY AGERELATED TAUOPATHY

MIGUEL QUINTAS-NEVES ${ }^{1,2,3}$, Merilee Teylan ${ }^{4}$, Rafaela MoraisRibeiro $^{2,3}$, Francisco Almeida ${ }^{2,3}$, Charles Mock ${ }^{4}$, Walter Kukull ${ }^{4}$, John F. Crary ${ }^{5}$, Tiago Gil Oliveira ${ }^{1,2,3}$

${ }^{1}$ Division of Neuroradiology, Hospital de Braga, Braga, Portugal, ${ }^{2}$ Life and Health Sciences Research Institute (ICVS), School of Medicine, University of Minho, Braga, Portugal, ${ }^{3}$ ICVS/3B's-PT Government Associate Laboratory, Braga/Guimaraes, Portugal, ${ }^{4}$ Department of Epidemiology, National Alzheimer's Coordinating Center, University of Washington, Seattle, Washington, ${ }^{5}$ Neuropathology Brain Bank \& Research Core, Department of Pathology, Nash Family Department of Neuroscience, Friedman Brain Institute, Ronald M. Loeb Center for Alzheimer's Disease, Icahn School of Medicine at Mount Sinai, New York

Keywords: Primary age-related tauopathy, Alzheimer's disease, MRI, brain imaging

Primary age-related tauopathy (PART) is a neuropathological designation characterized by the presence of Alzheimer's-type neurofibrillary tangles (NFTs) without major amyloid-beta $(\mathrm{A} \beta)$ deposition, while Alzheimer's disease (AD) is characterized pathologically by the accumulation of $A \beta$ plaques and NFTs. Although AD has been extensively characterized at the clinical and pathology levels, less is known regarding PART.

Our study aimed to compare the premortem in vivo brain MRI findings with neuropathological findings in patients with PART and AD, using visual rating regional atrophy scales, while assessing the relationship between brain atrophy and the degree of clinical impairment in all groups, based on the global Clinical Dementia Rating (CDR $®)$ Dementia Staging Instrument.

We analysed 245 participants: 44 with no plaques ("definite" PART CERAD 0), and 201 cases within the AD spectrum, specifically 25 with sparse (CERAD 1), 76 with moderate (CERAD 2), and 100 with severe (CERAD 3) neuritic plaques. Upon correcting for the effect of age, there was a statistically significant difference in brain atrophy on the anterior temporal (AT) region between groups CERAD 0 and CERAD $1(\mathrm{p}=0.04)$ and CERAD 1 and CERAD 3 ( $\mathrm{p}=0.005$ ). In the medial temporal region, significant differences between groups CERAD 0 and CERAD 3 $(\mathrm{p}=0.028)$, CERAD 1 and CERAD $3(\mathrm{p}=0.0002)$, and CERAD 2 and CERAD $3(p=0.012)$ were also found. We then assessed the correlation between regional brain atrophy scores and global CDR score for PART (CERAD 0) and AD (CERAD 1-3). We found that overall cognition deficits are directly correlated with regional atrophy in $\mathrm{AD}$, but not in PART.

These results indicate that PART and AD show different atrophy patterns, which correlate poorly with global cognitive deficits in PART. Overall, these findings support PART as an independent pathologic process from AD.

\section{1-O7}

EARLY DIFFUSION-WEIGHTED MRI AT 3TESLA DETECTS ISCHEMIC CHANGES OF THE OPTIC NERVE IN ANTERIOR ISCHEMIC OPTIC NEUROPATHY

SANDY MOURNET ${ }^{1}$, Thomas Sene ${ }^{2}$, Frederique Charbonneau ${ }^{1}$, Guillaume Poillon ${ }^{1}$, Catherine Vignal ${ }^{3}$, Gaelle Clavel$^{2}$, Jessica Guillaume $^{4}$, Julien Savatovsky ${ }^{1}$, Augustin Lecler ${ }^{1}$

${ }^{1}$ Department of Neuroradiology, Foundation Adolphe de Rothschild Hospital, Paris, France, ${ }^{2}$ Department of Internal Medicine, Foundation Adolphe de Rothschild Hospital, Paris, France, ${ }^{3}$ Department of Neuroophthalmology, Foundation Adolphe de Rothschild Hospital, Paris, France, ${ }^{4}$ Department of Clinical Research, Foundation Adolphe de Rothschild Hospital, Paris, France

Keywords: optic neuropathy, anterior ischemic optic neuropathy, Diffusion Magnetic Resonance Imaging, Magnetic Resonance Imaging

Introduction: For adults over 50 years old, anterior ischemic optic neuropathy $(\mathrm{AION})$ is the most common acute optic neuropathy, the diagnosis of which remains challenging on MRI. The purpose of our study was to assess the impact of timing from visual symptoms' onset to completion of a diffusion-weighted (DW) 3T MRI to detect ischemic changes of the optic disc and optic nerve in AION patients.

Methods: This IRB-approved retrospective single-center study included 126 patients with AION who underwent a 3T MRI from January 2015 to May 2020. Two radiologists blinded to all data, individually analyzed imaging. Discrepancies were resolved by consensus with a senior neuroradiologist. The primary judgment criterion was the presence of restricted diffusion of the optic disc and/or the optic nerve assessed subjectively on the ADC maps. ADC values of the optic disc and the optic nerve were measured. The correlation between timing from visual symptoms' onset to MRI completion and both the restricted diffusion and the ADC values was determined using Spearman's correlation analysis. Optimal thresholds for the timing were calculated from receiver operating characteristic (ROC); we defined and compared two groups: the early MRI group (MRI performed within 5 days after the visual symptoms' onset), versus the late MRI group (MRI performed after more than 5 days from visual symptoms' onset).

Results: One hundred and twenty-six patients (47/126 [37.3\%] women and 79/126 [62.7\%] men, mean age 69.1 +/- 13.7 years) with AION were included. Restricted diffusion of the optic disc in eyes affected by AION was significantly more likely to be observed in the early MRI group than in the late MRI group: $35 / 49$ (71.4\%) eyes versus $3 / 83(3.6 \%)$ eyes, $\mathrm{p}<0.001$. ADC values of the pathological optic discs and optic nerves were significantly lower in the early MRI group than in the late MRI group: 0.61 [0.52-0.94] x $10-3 \mathrm{~mm} 2 / \mathrm{s}$ versus 1.28 [1.01-1.44] x 10 $3 \mathrm{~mm} 2 / \mathrm{s}, \mathrm{p}<0.0001$ and $0.74[0.61-0.88] \times 10-3 \mathrm{~mm} 2 / \mathrm{s}$ versus 0.89 $[0.72-1.10] \times 10-3 \mathrm{~mm} 2 / \mathrm{s}, \mathrm{p}<0.001$, respectively (Figures-1,2).

Discussion: Our study showed a correlation between successfully detecting ischemic changes in AION and the timing from the onset of AION to the completion of MRI. Restricted diffusion of the optic disc was observed in a majority of eyes with AION in the early MRI group, but only rarely in the late MRI group.

Conclusions: DWI MRI showed good diagnostic performance to detect AION when performed early after the onset of visual symptoms. 


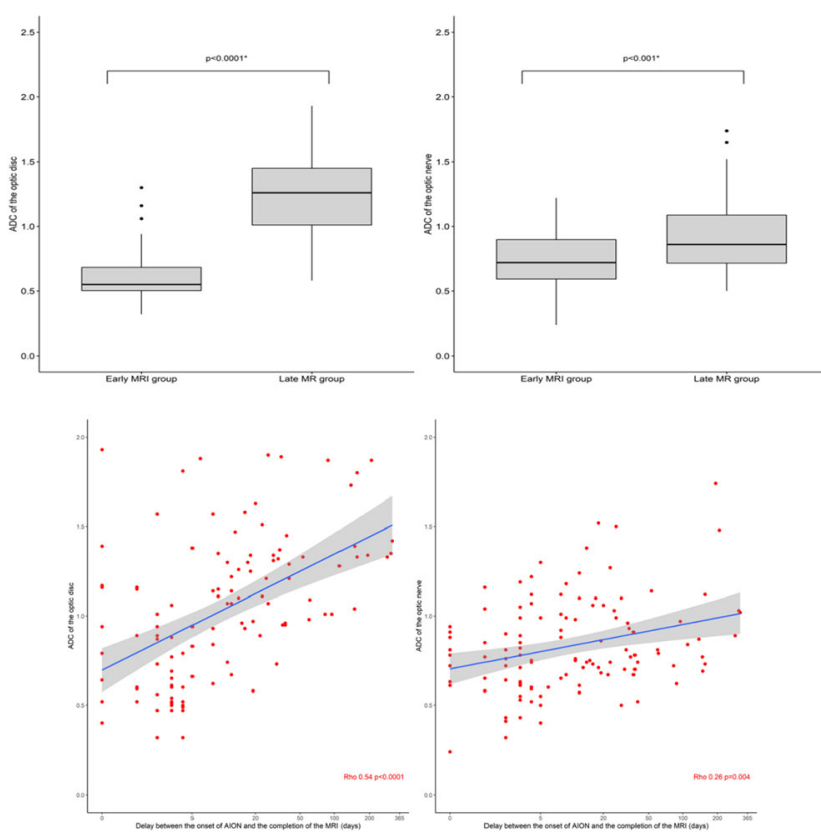

1-08

THIRD MONTH MRI PREDICTS MACROPROLACTINOMA REDUCTION AFTER CABERGOLINE THERAPY

YIKEN KARELYS NG WONG ${ }^{1}$, Silvana Sarria ${ }^{1}$, Alex Rovira ${ }^{1}$, Cristina Auger $^{1}$, Elena Martinez-Saez ${ }^{3}$, Ana Casteras ${ }^{2}$, Esteban Cordero ${ }^{4}$, Efrain Cordero $^{2}$, Irene Hernandez ${ }^{2}$, Alba Rojano ${ }^{1}$, Betina Biagetti ${ }^{2}$

${ }^{1}$ Neuroradiology Unit. Hospital Vall d'Hebron. Barcelona, Spain, ${ }^{2}$ Diabetes and Metabolism Research Unit, Vall D'Hebron Research Institute and CIBERDEM (ISCIII), Universidad Autonoma de Barcelona, Barcelona, Spain, ${ }^{3}$ Pathology Unit. Hospital Vall d'Hebron. Barcelona, Spain, ${ }^{4}$ Neurosurgery Unit. Hospital Vall d'Hebron. Barcelona, Spain

Keywords: MACROADENOMA, prolactinoma, cabergoline therapy, PITUITARY mri

Background: Transsphenoidal surgery is in general the preferred first-line treatment for patients with macroadenomas except for prolactinomas, which is mainly indicated when, the treatment with dopamine agonist (DA) fails. However, in those patients resistant to DA (i.e. they will not reduce the volume $>50 \%$ in the follow-up) this strategy delays the surgical procedure.

Objective: To identify predictors of volume reduction resistance, to select patients who may benefit from early surgery.

Methods: We retrospectively analysed a database of a tertiary reference centre searching for patients with active follow up in the last five years diagnosed with prolactinoma after 2010 (when medical records were computerized).

Results: A total of 185 prolactinomas where included: $124(67.0 \%)$ were microadenomas and 61 (33.0\%) were macroadenomas. We excluded 28 patients diagnosed before 2010 or with incomplete data and 6 patients that underwent surgery in first line. Finally, 27 patients meet de inclusion criteria; mean age [44.4 years; CI 95\%: (37.8-59.8)].), of whom 10 were women $(37.0 \%)$ and 17 were men $(63.0 \%)$. Mean follow up [67.5 months; CI 95\%: (52.5.0- 82.7)]. Ten $(37.0 \%)$ patients (8 males: $80.0 \%$ ) underwent surgery after more than one year of DA because a lack of tumour reduction. We used spearman rank to investigate if our main variable: reach a $50 \%$ of volume reduction along 12 months of CBG therapy, was influenced by several variables including age, gender, prolactin levels, cabergoline (CBG) doses and adenoma volume reduction at 3-4 month of follow-up. Multiple logistic regression analysis shown that the volume reduction at the first MRI (3-4 months) was the unique valuable predictor: [OR: 1.16 (IC 95\% 1.02-1.32)]. A cut off tumour volume reduction $\geq 35 \%$ by the first 3-4 months of CBG therapy predicted subsequent volume reduction $>50 \%$ in the first year with an AUC 0.95 [CI: (0.76-0.99)].

Conclusion: The tumor shrinkage in the first 3-4 month after starting treatment with DA is the best predictor of future volume reduction resistance. These results will help in decision-making regarding the management of prolactinomas.

\section{1-09}

SINGLE-VISIT MRI-DRIVEN STRATIFICATION OF MULTIPLE SCLEROSIS PATIENTS USING UNSUPERVISED MACHINE LEARNING

GIUSEPPE PONTILLO $^{1,2}$, Stefano Marrone $^{2}$, Sirio Cocozza ${ }^{1}$, Maria Petracca $^{3}$, Michela Gravina ${ }^{2}$, Simone Penna $^{2}$, Roberta Lanzillo ${ }^{3}$, Teresa Costabile $^{4}$, Daniele Riccio ${ }^{2}$, Andrea Elefante ${ }^{1}$, Mario Quarantelli ${ }^{5}$, Vincenzo Brescia Morra ${ }^{3}$, Carlo Sansone ${ }^{2}$, Arturo Brunetti ${ }^{1}$

${ }^{1}$ Department of Advanced Biomedical Sciences, University "Federico II", Naples, Italy, ${ }^{2}$ Department of Electrical Engineering and Information Technology (DIETI), University "Federico II", Naples, Italy, ${ }^{3}$ Department of Neurosciences and Reproductive and Odontostomatological Sciences, University "Federico II", Naples, Italy, ${ }^{4}$ Multiple Sclerosis Centre, II Division of Neurology, Department of Clinical and Experimental Medicine, "Luigi Vanvitelli" University, Naples, Italy, ${ }^{5}$ Institute of Biostructure and Bioimaging, National Research Council, Naples, Italy

Keywords: Multiple Sclerosis, Volumetric MRI, Unsupervised Machine Learning

Introduction: We used SuStaIn (Subtypes and Stages Inference), a recently developed unsupervised machine learning algorithm, to analyze brain MRI scans of Multiple Sclerosis (MS) patients and stratify them according to volumetric features. Our aim was to prove the biological reliability and clinical relevance of this approach.

Methods: We collected baseline and longitudinal 3T brain MRIs of relapsing remitting MS patients including 3D-T1w and FLAIR-T2w sequences, along with EDSS scores and long-term $(10 \pm 2 \mathrm{y})$ clinical outcomes (EDSS, cognition and transition to progressive course). Volumes of demyelinating lesions (TLV) and 116 atlas-defined GM regions were automatically segmented on structural MRI images and expressed as zscores referenced to an external population of healthy subjects. From baseline MRI scans, features that were mostly altered in MS patients were selected and fed into the SuStaIn algorithm, which estimates a set of subtypes characterized by distinct patterns of biomarker evolution, assigning each subject to a specific subtype and stage. The trained model was then applied to longitudinal MRIs.

The stability of SuStaIn subtypes over time and the annualized change of SuStaIn stage were assessed via Krippendorf's $\alpha$ and multilevel linear regression models, respectively, while the clinical relevance of the SuStaIn classification was assessed with ordinal/logistic regression analyses. 
Results: A total of 425 MS patients were selected, corresponding to 1129 MRI visits. Based on 11 biomarkers defined by feature selection (TLV and volumes of $10 \mathrm{GM}$ regions), two subtypes were identified, designated as "deep gray matter (DGM)-first" subtype (N=238) and "cortex-first" subtype $(\mathrm{N}=187)$ in the light of the initial atrophy pattern.

Disease subtypes were consistent over time $(\alpha=0.806)$, with significant annual increase in disease stage $(b=0.20 ; p<0.001)$. Baseline EDSS correlated with SuStaIn stage $(b=0.042 ; p<0.001)$ and the DGM-first subtype $(b=-0.280 ; p=0.02)$, with baseline stage also predicting long-term disability $(b=0.030 ; p=0.007)$ and transition to progressive course $(b=0.079 ; p=0.03)$. Long-term cognitive impairment was associated with baseline stage $(b=0.048 ; p<0.001)$ and the DGM-first subtype $(b=-$ $0.442 ; \mathrm{p}=0.005$ ).

Discussion \& Conclusion: Unsupervised learning modelling of volumetric features derived from clinical brain MRI scans provides a biologically reliable and prognostically meaningful stratification of MS patients, with possible relevant implications for both clinical trials and routine clinical practice.

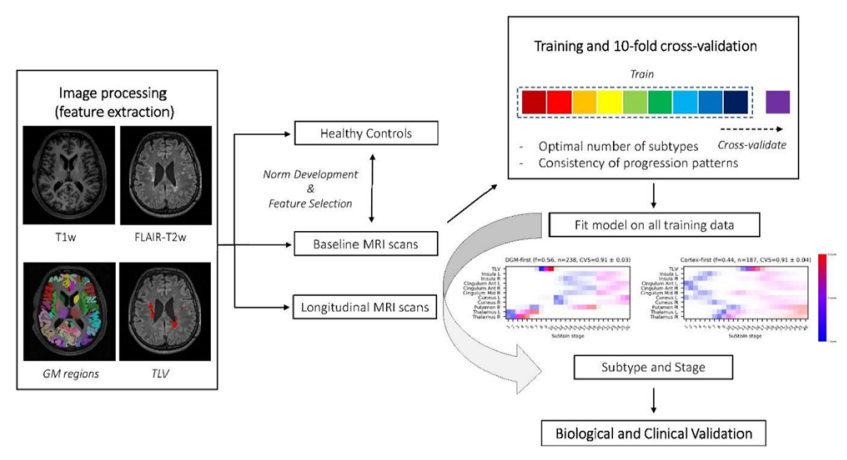

\section{1-O10}

AUGMENTED INTELLIGENCE: A HYBRID STRATEGY FOR GENERATING HIGH QUALITY GLIOBLASTOMA SUB-REGION SEGMENTATIONS USING BOTH COMPUTERS AND HUMAN EXPERTS

$\underline{\text { SATYAM GHODASARA }}^{1}$, Ujjwal Baid ${ }^{1,2,3}$, Spyridon Bakas ${ }^{1,2,3}$, Michel Bilello, ${ }^{1,2}$, Suyash Mohan ${ }^{1,2}$

${ }^{1}$ Department of Radiology, Perelman School of Medicine at the University of Pennsylvania, Philadelphia, PA, USA, ${ }^{2}$ Center for Biomedical Image Computing and Analytics, University of Pennsylvania, Philadelphia, PA, USA, ${ }^{3}$ Department of Pathology \& Laboratory Medicine, Perelman School of Medicine at the University of Pennsylvania, Philadelphia, PA, USA

Keywords: automated segmentation, brain tumor, glioblastoma, brats

Introduction: Artificial intelligence (AI) has been highly regarded as a groundbreaking tool to dramatically improve diagnostic methods in neuro-oncologic imaging and contribute to patient management by automatically analyzing pre-operative MRI scans with minimal human input. A common, valuable task for AI involves compartmentalizing glioblastoma into distinct sub-regions, i.e., necrotic core, enhancing tumor, peritumoral T2/FLAIR signal abnormality (ED). Manual segmentation of these sub-regions by expert neuroradiologists is impractical, requiring hours for intricate cases. Computer-aided segmentation (CAS) using AI can mitigate this issue but is often limited in the quality of the segmentations it produces. We hypothesize that a hybrid strategy employing AIpowered CAS followed by manual expert refinements is more practical and time-efficient than solely relying on either CAS or manual expert refinement alone.

Methods: CAS using an AI algorithm was used to generate tumor subregion segmentations on a total of 359 glioblastoma patients with four MRI sequences (T1, T1Gd, T2, T2-FLAIR) from each patient. All of the CAS-generated segmentations were sent to expert volunteer neuroradiologist annotators for manual refinements. Once refined, our team including two senior attending neuroradiologists with $>13$ years of experience each, reviewed and either approved or returned the segmentations to individual volunteer annotators for further refinements. Total time required to refine and review the finalized segmentations was measured.

Results: Following one round of refinements by expert volunteer annotators of the CAS-generated tumor sub-region segmentations, 244/359 (68\%) segmentations were approved by our team while 115/359 (32\%) segmentations contained a variety of errors that required a second round of refinements by the expert annotators. The most common observed errors were 1) missed ED in the anterior/inferior temporal lobes and corpus callosum (37/115 cases, $32 \%)$ and 2 ) erroneous segmentation of normal choroid plexus and blood vessels (14/115 cases, $12 \%)$. The expert annotators required 120 hours to refine all 359 segmentations, and our team required 26 additional hours to review them, resulting in 24 minutes/ segmentation following CAS.

Discussion \& Conclusion: Our findings support the value of a wellcommunicated hybrid annotation protocol to coordinate CAS and expert annotators. With CAS, our team and expert annotators rapidly finalized segmentations for 359 glioblastoma patients, demonstrating the value of a hybrid approach to creating high quality tumor sub-region segmentations.

\section{1-011}

GADOLINIUM: IS THE DEPOSIT REVERSIBLE? 1-YEAR MRI FOLLOW-UP IN A MULTIPLE SCLEROSIS PATIENT COHORT

FEDERICO BRUNO $^{1}$, Emanuele Tommasino ${ }^{1}$, Alessio Gagliardi ${ }^{1}$, Valeria Pagliei $^{1}$, Leonardo Pertici ${ }^{1}$, Alessandra Splendiani ${ }^{1}$

${ }^{1}$ Department of Biotechnology and Applied Clinical Sciences, University of L'Aquila, L'Aquila, Italy

Keywords: gadolinium, multiple sclerosis, MRI

Purpose: Gadolinium-based contrast agents (GBCAs) are widely used for everyday diagnostic procedures. Since 2014, numerous clinical studies demonstrated increased signal intensity (SI) on unenhanced T1weighted MRI images in dentate nucleus (DN) and globus pallidus (GP) after repeated administrations of linear GBCAs. This study aims to evaluate whether a washout of Gd from the dentate nucleus (DN) is possible in vivo after stopping macrocyclic GBCAs administrations in patients affected by relapsing-remitting multiple sclerosis (RR-MS) with visible T1-hyperintensity. Moreover, if it occurs, how quickly the signal intensity (SI) on unenhanced T1-weighted MRI images starts to decrease.

Methods \& Materials: We retrospectively analyzed 41 RR-MS patients with visible T1-hyperintensity of the DN after at least two administrations of macrocyclic GBCAs. Two independent Neuroradiologists blindly analysed all the unenhanced T1-weighted sequences of the subsequent MR follow-up and the relative time intervals to observe the trend of the visible $\mathrm{DN}$ hyperintensity and of the ND\P ratio.

Results: 9 patients out of 41 (22\%) did not show a significant variation of the visible T1-hyperintensity on the subsequent follow-up MRI examinations whereas 32 out of 41 (78\%) displayed a reduction of the visible T1- 
hyperintensity, and this was significant $(\mathrm{p}<0,05)$. In addition, statistically significant $(\mathrm{p} \leq 0.0004)$ were also the quantitative differences in mean $\mathrm{DN} / \mathrm{P}$ ratio for all patients in which visible T1-hyperintensity reduction was noted.

Conclusions: The reduction of visible T1 hyperintensity and DN/P SI ratio is time related. We identified a reduction of visible T1 hyperintensity and DN/P SI ratio in 32 of the 41 patients with RR-MS who developed visible hyperintensity after administrations of macrocyclic GBCAs. However, this reduction did not occur in patients in whom the time elapsed between the onset of $\mathrm{T} 1$ hyperintensity and the subsequent control without macrocyclic GBCAs was less than 12 months.

\section{1-012}

INTER-READER AGREEMENT FOR ASSESSING THE CIRCLE OF WILLIS ANATOMY IN PATIENTS WITH ACUTE ISCHEMIC STROKE USING TIME OF FLIGHT MAGNETIC RESONANCE ANGIOGRAPHY

NIKLAS LOHAUS ${ }^{1}$, Laura Philine Westphal ${ }^{2}$, Christian Manzolini ${ }^{2}$, Ulrike Held $^{3}$, Klaus Steigmiller ${ }^{3}$, Janne M. Hamann ${ }^{2}$, Andreas Luft ${ }^{2}$, Urs Fischer ${ }^{4}$, Johannes Kaesmacher ${ }^{5,6}$, Jan Gralla ${ }^{6}$, Marcel Arnold ${ }^{4}$, Susanne Wegener ${ }^{2}$, Roland Wiest ${ }^{6}$, Sebastian Winklhofer ${ }^{7}$

${ }^{1}$ Institute of Diagnostic and Interventional Radiology, University Hospital Zurich, Switzerland, ${ }^{2}$ Department of Neurology, University Hospital Zurich, Switzerland, ${ }^{3}$ Epidemiology, Biostatistics and Prevention Institute, Department of Biostatistics, University of Zurich, Switzerland, ${ }^{4}$ Department of Neurology, University Hospital Berne, Switzerland, 5 Department of Diagnostic, Interventional and Pediatric Radiology, University Hospital Berne, Switzerland, ${ }^{6}$ Department of Neuroradiology, University Hospital Berne, Switzerland, ${ }^{7}$ Department of Neuroradiology, Clinical Neuroscience Center, University Hospital Zurich, Switzerland

Keywords: Circle of Willis, magnetic resonance angiography., Interreader agreement, MRI, anatomy

Purpose: Assessing the Circle of Willis (CoW) anatomy and integrity is an essential part in acute ischemic stroke imaging. Time of flight magnetic resonance angiography (TOF-MRA) is a standard technique to evaluate intracranial vessels. Until now, there is only little knowledge about the test validity in ratings of the CoW using TOF-MRA. Therefore, aim of this study was to assess the inter-reader agreement for the analysis of the CoW in TOF-MRA.

Method \& Materials: Two readers independently classified the CoW anatomy based on TOF-MRA in 193 patients with acute ischemic stroke and middle cerebral artery M1-segment-occlusion. Within the CoW we qualitatively (presence, absence or hypoplasia) and quantitatively (diameter in $\mathrm{mm}$ ) evaluated the A1-segment of the anterior cerebral artery (A1), anterior communicating artery (Acom), P1-segment of the posterior cerebral artery $(\mathrm{P} 1)$, posterior communicating arteries (Pcom) and corresponding variants in each patient. Inter-reader agreement was calculated using Krippendorff's Alpha (K.A.) statistics. Intraclass correlation coefficient (ICC) and Cohens Kappa (K) were calculated for comparison purposes.

Results: An incomplete CoW (at least one vessel absent or hypoplastic) was found in $82 \%$ of patients. Inter-reader agreement for the ordinal variables of the segments was moderate (K.A. 0.41-0.79; к 0.38-0.81) and for the continuous variables of the segments slightly better (K.A. 0.46-0.82; ICC 0.49-0.82). Inter-reader-agreement was worst when categorizing Acom (K.A. 0.41 (CI 0.25-0.54); к 0.38 (CI 0.24-0.51)) and Pcom (right: K.A. 0.57 (CI 0.44-0.68); к 0.55 (CI 0.43-0.66); left: K.A. 0.61 (CI 0.49-0.71); к 0.59 (CI 0.48-0.71)). Pcoms demonstrated a relatively high inter-reader agreement for continuous variables (right: K.A. 0.73 (CI 0.64-0.81), ICC 0.74 (CI 0.63-0.81); left: K.A. 0.82 (CI 0.75-0.87), ICC 0.82 (CI 0.75-0.87)).

Conclusion: The inter-reader agreement for the CoW anatomy with TOF-MRA depends on the assessed vessel and is limited in particular for Acom and Pcom arteries. Inter-reader agreement for continuous variables is higher than for ordinal variables. These test limitations should be considered during stroke imaging evaluation.

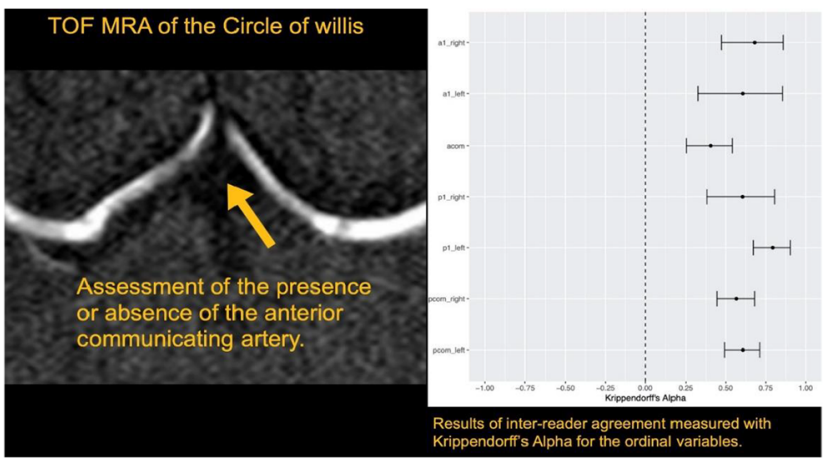

1-O13

ROLE OF PERFUSION MRI IN CLINICAL DECISION MAKING DURING BRAIN TUMOUR SURVEILLANCE

WOUTER TEUNISSEN ${ }^{1,2}$, Chee Wah Lam $^{3,4}$, Jeremy Labrecque ${ }^{3}$, Myriam Hunink $^{1,3}$, Marion Smits ${ }^{1,2}$

${ }^{1}$ Erasmus MC, Department of Radiology and Nuclear Medicine, Rotterdam, The Netherlands, ${ }^{2}$ Brain Tumour Centre, Erasmus MC Cancer Institute, Rotterdam, The Netherlands, ${ }^{3}$ Erasmus MC, Netherlands Institute for Health Science (NIHES), Rotterdam, The Netherlands, ${ }^{4}$ Erasmus MC, Department of Neurology, Rotterdam, The Netherlands

Keywords: Perfusion MRI, Brain tumour, Medical decision making

Introduction: One of the major challenges during brain tumour followup is the differentiation between tumour progression and treatment induced abnormalities. Conventional contrast enhanced T1W images cannot differentiate between progression and treatment induced abnormalities, which causes diagnostic uncertainty. Often perfusion MRI (pMRI) is added to the standard protocol to resolve this issue. As pMRI comes with higher costs due to longer acquisition times, contrast use, post-process and interpretation, it is important to evaluate the clinical impact of the usage of this radiological technique. Currently, there is no consensus on the best usage of pMRI and it is still not well known whether the use of pMRI affects treatment decisions. The aim of this study is to evaluate the role of pMRI on clinical decision making.

Methods: We performed a single centre retrospective cohort study of all patients treated for brain tumours (both glioma and metastasis) between 2008-2018. Clinical data were collected from electronic health records, identifying the first scan after treatment with significant increase of enhancement or $\mathrm{T} 2 \mathrm{~W}$ abnormalities. Then, the treatment decision based on the results of that scan was collected. If pMRI was performed, its quality and outcomes were reported. We considered cases with pMRI but without any form of interpretation as no pMRI cases. We chose confounders based on a causal graph (table 1) and analysed the data using a logistic regression model. Subsequently we estimated the probability that pMRI led to a different treatment decision. 
Results: We analysed 469 patients with glioma (308 male, $56.8 \pm 12.4 \mathrm{yr}$ ) and 124 with metastasis ( 47 male, $60.5 \pm 10.5 \mathrm{yr}$ ). Table 2 and 3 show the characteristics of the variables used in the model (except Karnofsky Performance Status). We found that $-11.1 \%$ (CI: -20.9\%, -1.43\%) of post-scan treatment decisions in patients with glioma would be different if pMRI had been performed in addition to the standard protocol. In patients with metastasis, $-21.5 \%$ (CI: $-48.6 \%, 4.46 \%$ ) of post-scan treatment decisions would be different with pMRI.

Discussion \& Conclusion: These preliminary results demonstrate reduced treatment change in a small proportion of patients based on the follow-up scan with pMRI compared to those without pMRI. These results indicate a role for pMRI in treatment decisions of patients with a brain tumour during surveillance after treatment. The effect is small and possibly due to residual and unmeasured confounding.
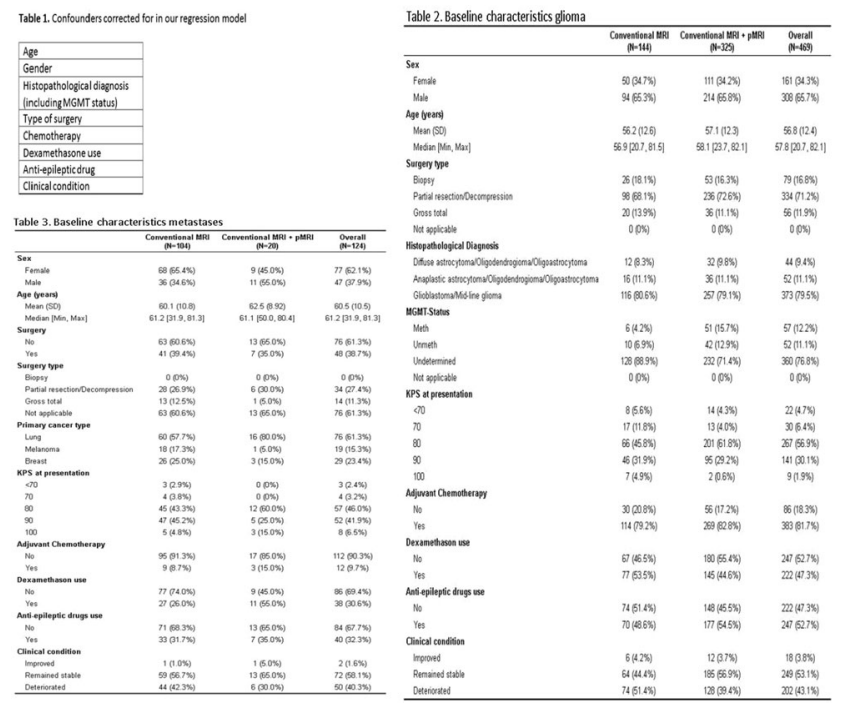

\section{1-014}

ASSESSMENT OF THE EVOLUTION OF CTP BASED PENUMBRA AFTER SUCCESSFUL ENDOVASCULAR RECANALIZATION FOR INTRACRANIAL LARGE VESSEL OCCLUSION

BENCE NEMETH $^{1}$, Leyla Acu ${ }^{1}$, Patrick Thurner ${ }^{1}$, Jawid Madjidyar ${ }^{1}$, Sebastian Winklhofer ${ }^{1}$, Lars Michels ${ }^{1}, Z_{\text {Zsolt Kulcsar }}{ }^{1}$, Tilman Schubert ${ }^{1}$

${ }^{1}$ Department of Neuroradiology, Clinical Neuroscience Center, University Hospital Zurich, University of Zurich, Zurich, Switzerland

Keywords: Stroke, Penumbra, Thrombectomy

Introduction: Imaging of the ischemic penumbra remains a challenging task. It is likely that the degree of collateralization not only defines the penumbra at the time of imaging, but also the temporal evolution of the penumbra (1). In a number of perfusion imaging softwares, penumbra is defined as Tmax $>6$ s. However, perfusion imaging cannot yet predict penumbra evolution. In this study, we explore the interactions between extended Tmax times $(>8 \mathrm{~s},>10 \mathrm{~s})$ as well as time to recanalization with final infarct core volume in a cohort of patients with large vessel occlusion that underwent thrombectomy. All patients were successfully recanalized (TICI IIc-III).

Methods: 55 patients with large intracranial artery occlusions who underwent mechanical thrombectomy who received preinterventional CT, CT angio-graphy (CTA) and CT perfusion (CTP) were included.
Rapid was used for CTP processing (RapidAI, USA). Final infarct volume was segmented on postinterventional diffusion-weighted MR-imaging acquired within five days (average 1,75 days). Explorative statistics: We assessed symptom-onset to recanalization (TR), ischemic penumbra (Tmax $>6 \mathrm{~ms})$, extended tmax $(\mathrm{T} \max >8 \mathrm{~ms}$, Tmax $>10 \mathrm{~ms})$ volumes and CTP based initial infarct core (preIC). In this preliminary analysis, we performed Pearson correlation analyses to examine the interaction between the assessed variables and the final infarct core volume.

Results: Average and standard deviation values are summarized in table 1. The Pearson correlation yielded a significant correlation between Tmax $>6 \mathrm{~ms}$ with finalIC $(\mathrm{r}=0.55, \mathrm{p}<0.0001)$, Tmax $>8 \mathrm{~s}$ with finalIC $(r=0.61, p<0.0001)$ and Tmax $>10$ s with finalIC $(r=0.59, p<0.0001)$. All correlations are summarized in fig. 1. In addition, a strong correlation was found between the size of CTP based preoperative infarct core and postoperative final core volume $(r=0.87, \mathrm{p}<0.0001)$. Symptom-onset to recanalization yielded no significant correlation with finalIC in this cohort.

Discussion \& Conclusion: In this study, we explored the interactions of extended CTP based Tmax times and final infarct core in patients with large vessel occlusion which were successfully recanalized. We found significant correlations between $T \max >6 \mathrm{~s}$, Tmax $>8 \mathrm{~s}$ and Tmax $>10 \mathrm{~s}$ with the highest correlation at $T \max >8 \mathrm{~s}$. This may indicate that patients with a large volume of extended Tmax are at increased risk to develop a larger infarct after recanalization.

References: Leigh et al, Imaging the physiological evolution of the ischemic penumbra in acute ischemic stroke. Cereb Blood Flow Metab. 2018 Sep;38(9):1500-1516. doi: 10.1177/0271678X17700913. Epub 2017 Mar 27.

Table 1: Descriptive statstics of all variables

\begin{tabular}{|c|c|c|c|c|c|c|c|}
\hline Features & prelc & tmax4 & penumbra & tmax8 & tmax10 & TR & finallc \\
\hline Description & $\begin{array}{c}\text { initial } \\
\text { infarct core }\end{array}$ & $\begin{array}{c}\text { Volume: Tmax } \\
>4 \text { ms }\end{array}$ & $\begin{array}{c}\text { Volume: Tmax } \\
>6 \text { ms }\end{array}$ & $\begin{array}{c}\text { Volume: Tmax } \\
>8 \mathrm{~ms}\end{array}$ & $\begin{array}{c}\text { Volume: Tmax } \\
>4 \mathrm{~ms}\end{array}$ & $\begin{array}{c}\text { Time to } \\
\text { recanalization }\end{array}$ & $\begin{array}{c}\text { final infarct } \\
\text { core volume }\end{array}$ \\
\hline mean & 17.86 & 212.59 & 113.90 & 69.59 & 44.93 & 182.34 & 23.84 \\
\hline SD & 34.15 & 109.94 & 79.88 & 68.27 & 54.31 & 125.41 & 31.90 \\
\hline $\min$ & 0 & 33 & 5 & 0 & 0 & 24 & 0 \\
\hline $25 \%$ & 0 & 145 & 65 & 26 & 7 & 124 & 5.37 \\
\hline $50 \%$ & 6 & 194 & 110 & 62 & 35 & 172 & 13.93 \\
\hline $75 \%$ & 19 & 241 & 128 & 85 & 59 & 197 & 23.66 \\
\hline $\max$ & 168 & 541 & 415 & 339 & 253 & 760 & 119.26 \\
\hline
\end{tabular}

Table 2: Pearson correlation coefficients between evaluated variables correlation values

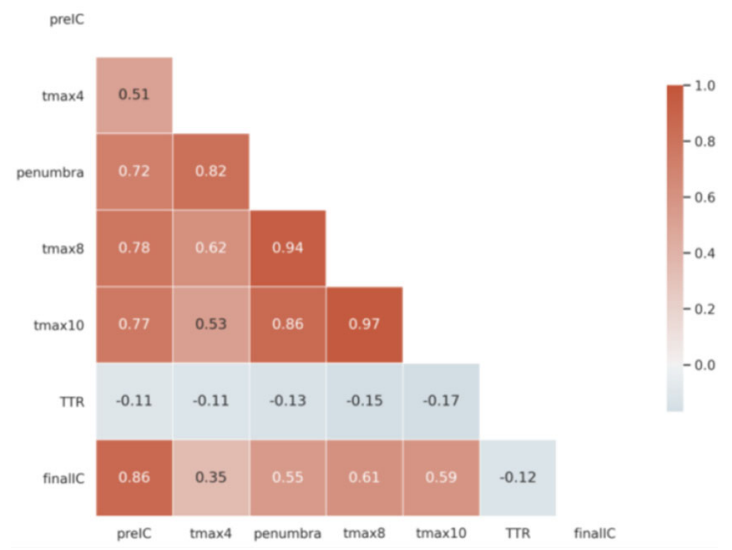


1-015

LONG-COVID: HYPER CONNECTIVITY OF SALIENCE NETWORK IN COVID CRITICALLY-ILL PATIENTS

ALBERTO CABRERA-ZUBIZARRETA ${ }^{1,2}$, Antonio Jimenez-Marin ${ }^{1,3}$, Daniela Ramos-Usuga ${ }^{3,4}$, Itziar Benito-Sanchez ${ }^{3,4}$, Endika MartinezGutierrez $^{1,5}$, Victoria Boado ${ }^{6}$, Fermin Labayen ${ }^{6}$, Juan Carlos ArangoLasprilla $^{4,7,8}$, Jesus Maria Cortes ${ }^{1,7,8}$

${ }^{1}$ Computational Neuroimaging Group. Biocruces-Bizkaia Health Research Institute, Barakaldo, Spain, ${ }^{2}$ Osatek, Galdakao, Spain, ${ }^{3}$ Biomedical Research Doctorate Program, University of the Basque Country (UPV/EHU), Leioa, Spain, ${ }^{4}$ Psychology and Health Group. Biocruces-Bizkaia Health Research Institute, Barakaldo, Spain, ${ }^{5}$ Dipartimento Interateneo di Fisica, Universita degli Studi di Bari Aldo Moro, and INFN, Sezione di Bari, Italy, ${ }^{6}$ Intensive Care Unit. Cruces University Hospital, Barakaldo, Spaind, ${ }^{7}$ IKERBASQUE, The Basque Foundation for Science, Bilbao, Spain, ${ }^{8}$ Department of Cell Biology and Histology, University of the Basque Country (UPV/EHU), Leioa, Spain

Keywords: Long-Covid, Salience network, functional connectivity, resting state

Long-COVID, a.k.a. post-acute sequelae of COVID-19, is a poorly defined condition that encompasses fatigue, headaches and muscle aches, memory loss, sleep disorders and concentration problems and attention deficit, among other symptoms. We focused on brain connectivity alterations at rest in a cohort of $\mathrm{N}=15$ critically-ill COVID-19 patients as compared to $\mathrm{N}=15$ healthy controls, matched by age and sex. Imaging acquisitions were performed six months after Intensive Care Unit (ICU) discharge. Following a similar methodology as in (Jimenez-Marin et al, Neuroimage clinical, 2021), we built functional connectivity matrices at the voxel level (total number 50,125 voxels, mm3). Using masks of known resting state networks (RSN) (Yeo et al., J Neurophysiol., 2011), we obtained for each RSN and participant a 3D brain map of strength by summing across all $\mathrm{FC}$ values from any voxel in the brain towards all voxels within each RSN. Significant differences in salience connectivity were found after multiple-comparisons and for the contrast patient > control, indicating hyper-connectivity of the salience network (SN) in COVID-19 patients as compared to controls at six months after ICU. Hyper-connectivity occurred from the SN towards visuospatial $(40 \%)$, somatosensory $(35 \%)$, dorsal attention $(30 \%)$, ventral attention $(18 \%)$, limbic $(16 \%)$, executive frontoparietal $(13 \%)$ and default mode networks $(15 \%)$. There was a significativo difference in patient vs controls in 13254 voxels out of 50125 voxels, representing up to $26 \%$ of grey matter. SN has been implicated in the integration of emotional and sensory stimuli as well as in modulating the switch between the internally directed cognition of the default mode network (DMN) and the externally directed cognition of the central executive network (CEN). SN hyper connectivity has been described in anxiety disorders, post traumatic stress disorder, autism spectrum disorder and schizophrenia and may account for the neuropsychiatric symptoms in Long-Covid patients. For how long these alterations persist in long-COVID remains to be elucidated.
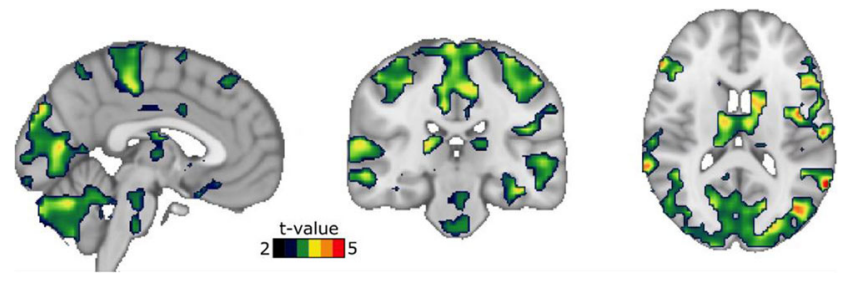

\section{1-016}

90 SECOND CT IMAGING PROVIDES SUPERIOR SNR AND CNR OVER DELAYED IMAGING OF BRAIN METASTASES

JASON MICHAEL JOHNSON $^{1}$, Abhi Rashiwala ${ }^{1}$, Brian Nan ${ }^{1}$, Apollo Krayyem $^{1}$, Ethan Wang ${ }^{1}$, Halyna Pokhylevych ${ }^{1}$, Ahmad Amer ${ }^{1}$, Hamza Alhasan ${ }^{1}$, Dawid Schellingerhout ${ }^{1}$, Sherise Desiree Ferguson ${ }^{2}$, Jing $\mathrm{Li}^{3}$, Rick Layman ${ }^{4}$

${ }^{1}$ Department of Neuroradiology, The University of Texas MD Anderson Cancer Center, Houston, USA, ${ }^{2}$ Department of Neurosurgery, The University of Texas MD Anderson Cancer Center, Houston, USA, ${ }^{3}$ Department of Radiation Oncology, The University of Texas MD Anderson Cancer Center, Houston, USA, ${ }^{4}$ Department of Imaging Physics, The University of Texas MD Anderson Cancer Center, Houston, USA

Keywords: metastasis, CT, contrast timing, diagnostic optimization

Brain metastases (BM) have been reported to occur in up to $40 \%$ of patients with cancer. At present, gadolinium-enhanced MRI is considered to be the imaging technique of choice in patients suspected of BM; however, CT is cheaper, more widely available, and does not suffer from geometric distortion as seen with MR imaging. Prior studies have shown superior conspicuity of brain malignancy with delayed imaging times. We sought to optimize CT parameters for BM conspicuity in a prospective trial.

23 patients ( 12 women, $58.4 \pm 14.6$ years) with $4.4 \pm 2.5$ BM were scanned on a DECT scanner (Somatom Force, Siemens, Malvern, USA) at 90 seconds, 5 minutes, 10 minutes, and 20 minutes following the injection of $123 \pm 8.0 \mathrm{ml}$ of iohexol (GE Healthcare, Chicago, USA). The primary cancer was melanoma (7), lung (7), breast (5), genitourinary (3), and gastrointestinal (1). A circular ROI was drawn on $4 \mathrm{~mm}$ axial QC images where the largest BM $(16.0 \pm 6.3 \mathrm{~mm}$ diameter $)$ had the largest crosssectional diameter. BM with necrosis also had an ROI placed to cover the area of maximal enhancement while avoiding tissue outside of the $\mathrm{BM}$ and the necrotic (non-enhancing) region. An ROI was also placed in the contralateral normal-appearing brain (NAB and in the air ("noise" measurement). ROIs were obtained at each imaging time point. For lesions with necrosis, the "hot spot" was used for SNR/CNR. A single-tail Students T-test was used for comparative statistics.

At $90 \mathrm{sec}$, the mean BM HU was $85.3 \pm 12.0$, SNR $27.0 \pm 10.0$, CNR 12.8 \pm 4.7 . At $5 \mathrm{~min}$, the mean BM HU was $80.4 \pm 13.3$, SNR $25.3 \pm 10.5$, CNR 11.9 \pm 5.9 . At $10 \mathrm{~min}$, the mean BM HU was $77.2 \pm 12.0$, SNR $22.5 \pm 6.7$, CNR 10.0 \pm 3.5 . At $20 \mathrm{~min}$, the mean BM HU was $74.0 \pm 9.8$, SNR 23.4 $\pm 7.1, \mathrm{CNR} 10.4 \pm 3.7$. CNR was greatest at $90 \mathrm{sec}$, and this difference was statistically significant $(\mathrm{P}<0.05)$ compared to 10 and $20 \mathrm{~min}$. CNR at 5 min was also statistically significantly greater than later time points $(\mathrm{P}<$ $0.05)$.

Despite prior reports demonstrating greater conspicuity for brain malignancy using later time points, our results show that earlier time points show objectively greater conspicuity. Additional work remains to verify that this difference leads to a difference in radiologist detection and comparison of different virtual monochromic reconstruction levels on BM conspicuity. 


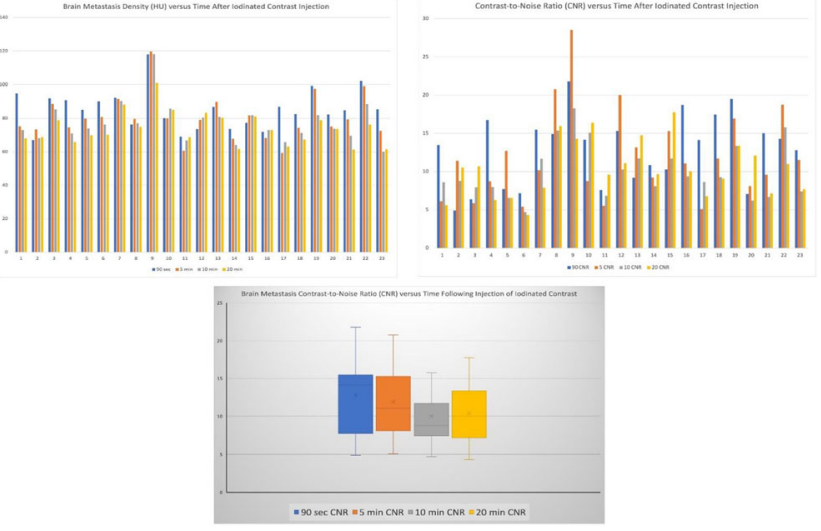

1-017

ULTRA-HIGH RESOLUTION COMPRESSED SENSING TOF-MRA FOR INTRACRANIAL ANEURYSMS

TILMAN SCHUBERT ${ }^{1}$, Markus Klarhoefer ${ }^{2}$, Bence Nemeth ${ }^{1}$, Zsolt Kulcsar $^{1}$, Shakir Husain Hakim ${ }^{1}$

${ }^{1}$ Department of Neuroradiology, University Hospital Zurich, Zurich, Switzerland, ${ }^{2}$ Siemens Healthineers, Erlangen, Germany

Keywords: Compressed Sensing, MR-Angiography, Intracranial aneurysms

Introduction: 3D time of flight (TOF) MRA shows a high sensitivity for the detection of intracranial aneurysms (1). At 3T, this modality is also helpful in planning of endovascular aneurysm treatment. However, the performance in complex or very small aneurysms is still inferior to DSA due to limited spatial resolution (2). In this study, we used compressed sensing (CS) reconstruction to achieve a higher resolution without increasing scan time and compared the diagnostic performance with conventional TOF-MRA and 3D-DSA.

Methods: 16 patients with conventional TOF at 3T, CS-TOF at 3T and 3D-DSA were included in this retrospective study. Informed consent was obtained. Average inplane resolution of conventional TOF was $0.6 \mathrm{x}$ $0.6 \mathrm{~mm}$ (matrix: 320/280, FOV 190/166) with a slice thickness of $0.6 \mathrm{~mm}$. Inplane resolution of CS-TOF was $0.4 \times 0.4 \mathrm{~mm}$ (matrix 544/ 494 (FOV: 220/200) with a slice thickness of $0.3 \mathrm{~mm}$. Image quality of TOF and and CS-TOF were graded on a four-point scale (excellent / good / sufficient / poor). All MRI scans and 3D-DSAs were independently evaluated for the number of aneurysms. Maximum and minimum aneurysm diameter, aneurysm depth and maximum neck width were measured on multiplanar reconstructions. 2-sided, paired t-test were applied to test for statistically significant differences.

Results: 22 aneurysms were detected on 3D-DSA, 22 on CS-TOF and 20 aneurysms on conventional TOF. All TOF and CS-TOF acquisitions were of excellent or good image quality. Average measurement results in mm for 3D-DSA, CS-TOF and conventional TOF are summarized in table 1. P-values of the performed student t-tests are included in table 1. Maximum neck width measurements of CS-TOF and 3D-DSA were statistically significantly different, all other measurements showed no significant differences.

Discussion \& Conclusion: The diagnostic performance of ultra-high resolution CS-TOF was excellent reflected by the correct depiction of all aneurysms that were found on 3D-DSA. Although image quality was excellent or good for all conventional TOF exams, two aneurysms were not depicted. One was a very small inferior wall of internal carotid artery aneurysm adjacent to a larger aneurysm (Fig. 1). The other one was a recurrent aneurysm next to a coil pack and microsurgical clip. Conversely, the only significantly different measurement result was maximum neck width between CS-TOF and 3D-DSA.

\section{Literature:}

1) Li et al, Accurate Diagnosis of Small Cerebral Aneurysms $5 \mathrm{~mm}$ in Diameter with 3.0-T MR Angiography, Radiology 2014

2) Chen et al, The clinical value of MRA at 3.0 T for the diagnosis and therapeutic planning of patients with subarachnoid haemorrhage, Eur Radiol 2012

Table 1: Average values are summarized. Minuscule $t$ refers to $p$-values of student t-tests between measurements

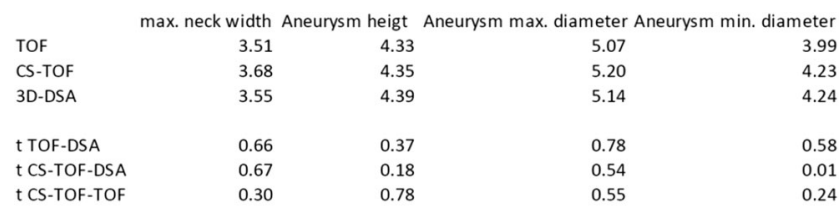

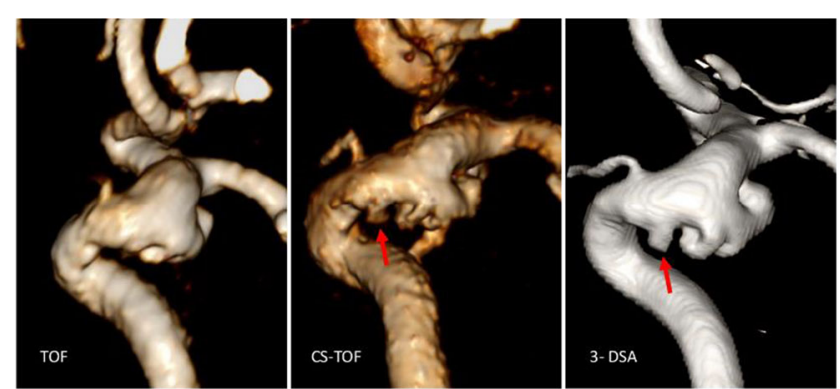

\section{INTERVENTIONAL}

\section{2-01}

DIRECT THROMBOASPIRATION EFFICACY ACCORDING TO THE ANGLE OF INTERACTION BETWEEN THE ASPIRATION CATHETER AND THE CLOT. CLINICAL AND IN-VITRO EXPERIENCES

GIANMARCO BERNAVA ${ }^{1}$, Andrea Rosi ${ }^{1}$, Jeremy Hofmeister ${ }^{1}$, Hasan Yilmaz $^{1}$, Karl Olof Loevblad ${ }^{1}$, Paolo Machi ${ }^{1}$

${ }^{1}$ Neuroradiology Unit, Hôpitaux universitaires Geneve, Geneva, Switzerland

Keywords: Thromboaspiration, Stroke, clot, Angle of interaction

Background: Direct thromboaspiration has been reported as an effective mechanical treatment for acute ischemic stroke. We aimed to determine whether the angle of interaction between the aspiration catheter and the clot affects the success of clot removal, through a clinical retrospective study and an in-vitro experience.

\section{Methods:}

Clinical study: all patients treated at our institution by direct thromboaspiration as a first line technique between January 2016 and December 2017 were enrolled in the study. We retrospectively reviewed baseline and procedural characteristics, the angle of interaction formed between the aspiration catheter and the clot, the modified Thrombolysis 
in cerebral infarction score, and the 3 months modified ranking scale score.

In-vitro study: A rigid vascular phantom designed to reproduce a middle cerebral artery trifurcation anatomy with three M2 segments forming different angles with M1 and synthetic clots of different consistencies and sizes was used.

Results: The clinical study showed that an angle of interaction of $\geq 125.5^{\circ}$ significantly influenced the success of clot removal $(\mathrm{P}<0.001)$ with good sensitivity and specificity, in particular for occlusion of the middle cerebral and basilar artery. Such finding was confirmed by the in-vitro study, irrespective of clot features. In both studies rescue stent retriever thrombectomy was a valid rescue treatment in cases of standalone direct thromboaspiration failure.

Discussion \& Conclusions: Our experience showed that an angle of interaction between the aspiration catheter and the clot of $\geq 125.5^{\circ}$ was significantly associated with successful clot removal. Stent retriever thrombectomy was an effective rescue treatment after direct thromboaspiration failure, even in the presence of an unfavorable angle of interaction or hard clots. The prediction of the angle of interaction on pretreatment imaging may help operators to select the most adequate mechanical thrombectomy technique on a case by case basis.

Figure 1: Schematics of vessel anatomy and example of angle of interaction (AOI). Light gray and dark gray depict the vessels before and after thromboaspiration, respectively. (A) The AOI is defined as the smallest angle $\left(\leq 180^{\circ}\right)$ between the clot (red) and the aspiration catheter (blue). (B) Example AOI on real DSA.

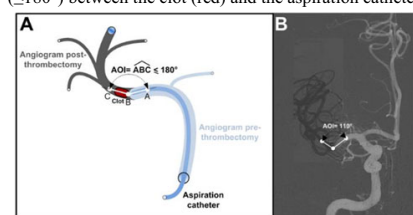

Figure 2: (A) Schematic representation of the vascular phantom used for the experiments, with vessel diameters and angles between $\mathrm{M} 1$ and $\mathrm{M} 2$ branches. (B) Thrombus analog location and catheter positioning for M1 occlusion experiments. (C) Location of thrombus

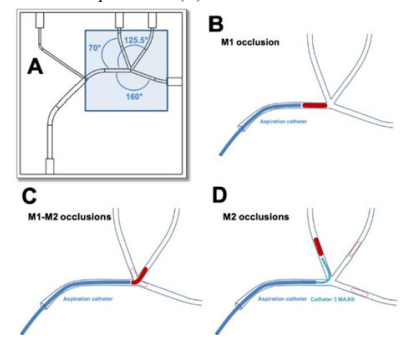

2-O2

ACUTE ENDOVASCULAR TREATMENT OF CAROTID DISSECTIONS: A SINGLE CENTER EXPERIENCE

SOFIA QUEIROS DE ALMEIDA XAVIER ${ }^{1}$, Pedro Bem Gomes ${ }^{2}$, Alberto Fior $^{3}$, Ana Paiva Nunes ${ }^{3}$, Isabel Fragata ${ }^{4}$, Joao Lopes dos Reis ${ }^{4}$

${ }^{1}$ Neuroradiology Department, Hospital de Braga, Braga, Portugal, ${ }^{2}$ Neuroradiology Department, Unidade de Saude Local, Matosinhos, Portugal, ${ }^{3}$ Cerebrovascular Unit, Centro Hospitalar Universitario de Lisboa Central, Lisboa, Portugal, ${ }^{4}$ Neuroradiology Department, Centro Hospitalar Universitario de Lisboa Central, Lisboa, Portugal

Keywords: carotid artery dissection, acute ischemic stroke, endovascular treatment
Introduction: Internal carotid artery dissection (ICAD) is a rare cause of stroke. Endovascular therapy (EVT) of acute ischemic stroke (AIS) after spontaneous ICAD is still controversial. Few studies conclude that EVT is safe and effective for treatment of ICAD, but the best technique remains to be determined. We report our experience in EVT of AIS due to spontaneous ICAD.

Methods: Data was obtained from retrospective analysis of consecutive patients with AIS and ICAD between January 2015 and March 2021. Exclusion criteria were extracranial ICA occlusion due to other cause (e.g. atheromatous, cardiogenic embolism); pre-stroke modified Rankin Scale (mRS) score $>2$ and age $<18$ years. Primary outcome was 90-day $\mathrm{mRS} \leq 2$. Secondary outcomes included: modified Thrombolysis in Cerebral Infarction (mTICI) score 2b-3, extracranial recanalization, procedural complications, symptomatic intracerebral hemorrhage (sICH) and 90-day mortality.

Results: We included 56 patients with ICAD: $23(41,1 \%)$ were treated with EVT alone, $33(58,9 \%)$ with combined IV tPA and EVT. Mean age was $50 \pm 10,5$ years, $58,9 \%(\mathrm{n}=33)$ were men, and the median admission National Institutes of Health Stroke Scale score was 14,2 (interquartile range, $10-19,5)$. Thirty-nine patients $(69,6 \%)$ had tandem occlusions, and in $97,4 \%(n=38)$ of these, mechanical thrombectomy (MT) with aspiration was performed. Some patients required stent retriever as rescue after aspiration failure $(18,4 \%, \mathrm{n}=7)$. ICAD was treated with balloon angioplasty in 30,4\% $(n=17)$. Carotid stents were used in $28,6 \%(n=16)$ cases, whenever a residual stenosis $>70 \%$ was noted after MT. In tandem lesions, successful reperfusion (mTICI $2 b-3)$ occurred in $97,4 \%(n=38)$. During ICAD endovascular approach, thrombus migration to the intracerebral circulation was observed in three patients that was effectively resolved with MT. There were no other intraprocedural complications. sICH occurred in $7,1 \%$ of patients $(n=4)$, malignant stroke in $7,1 \%$ $(n=4)$ and $8,9 \%(n=5)$ required decompressive craniectomy. Ten patients were lost to follow up at 90 days. Of the 46 patients followed $67,4 \%$ $(n=31)$ had favorable outcomes $(m R S \leq 2)$ and $2,2 \%$ died $(n=1)$.

Conclusion: In our cohort of patients with AIS after ICAD, we observed high rates of ICA and intracranial recanalization, mostly without the need of acute carotid stenting. Functional outcome at 90 days was favorable, and mortality was low, possibly reflecting the young age of patients and good prognosis of ICAD. Our results are in line with previous studies reporting a high safety and efficacy profile of EVT in the setting of acute ICAD.

\section{2-O3}

\section{HOW CLOTS EMBED ALONG THE STENT RETRIEVERS}

DANIELA DUMITRIU LA GRANGE ${ }^{1}$, Gianmarco Bernava ${ }^{1}$, Andrea Rosi $^{1}$, Jeremy Hofmeister ${ }^{1}$, Isabel Wanke ${ }^{2}$, Paolo Machi ${ }^{1,3}$, Karl-Olof Loevblad $^{1,3}$

${ }^{1}$ Neuroradiology, Klinik Hirslanden Zurich, Switzerland, ${ }^{2}$ Faculty of Medicine of Geneva University, Switzerland, ${ }^{3}$ Department of Radiology and Medical Informatics, University of Geneve, Switzerland

Keywords: stent retriever, clot, acute ischemic stroke

Introduction: Standard of care in acute ischemic stroke (AIS) involves endovascular treatment of large vessel occlusion using stent retriever (SR) thrombectomy. How clots embed along the stent retrievers is still a matter of investigation. In this study we aim to highlight, using scanning electron microscopy (SEM), how clot characteristics such as volume, composition and compactness relate to clot incorporation into the stent. 
Methods: The clots were retrieved from patients suspected of being victims of AIS, who received whole brain stroke CT protocol and were referred for mechanical thrombectomy intervention. For all patients, the clots were located within the segments M1-M2 of the middle cerebral artery (MCA). Thrombectomy procedures were performed according to standard clinical practice. The retrieved clots, attached on stents, were prepared for scanning electron microscopy (SEM) according to standard protocols. The microscopy observations were performed with an ultrahigh resolution field-emission Zeiss Merlin SEM.

Our results show that the stent struts are protruding through red clots. White clots are embedded along the stent through wrapping around the strut or by wetting the stent surface. We found a linear correlation $(\mathrm{R} 2=$ 0.9236) between the contact surface (calculated as clot volume - to - stent length covered by clot ratio) and the clot volume - Figure 1. We observed that the clots are less compact at the attachment sites onto the struts.

Figure 1: a) variation of extent of clot contact on stent with the clot volume, b) red blood clot on stent, c) white clot on stent.

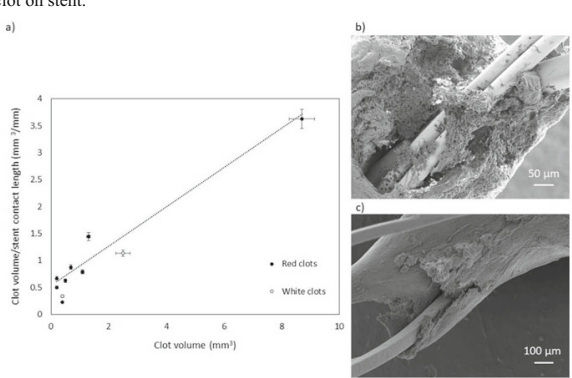

Discussion: We show that variations in compactness render the clots deformable and amenable to incorporation onto the stent. Clot composition, red blood cells rich or fibrin rich, is responsible for the attachment modality. As the clot volume increases, the relative contact surface is diminished.

Conclusion: White clots and red clots embed onto the stent on the account of volume regions with decreased compactness. We hypothesize that clot volume, composition and compactness modulation are responsible for the endovascular treatment outcome.

\section{HEAD \& NECK}

\section{3-O1}

CORRELATION BETWEEN CTA AND HISTOLOGY OF CAROTID ARTERY ATHEROSCLEROSIS: CAN SEMI-AUTOMATED IMAGING SOFTWARE PREDICT A PLAQUE'S COMPOSITION?

JOHN BENSON $^{1}$, Bradley J Erickson ${ }^{1}$, Laurence J. Eckel ${ }^{1}$, Felix E. Diehn $^{1}$, Greta Liebo ${ }^{1}$, Norbert G. Campeau ${ }^{1}$, Waleed Brinjikji ${ }^{1}$, Valentina Nardi ${ }^{2}$, Amir Lerman ${ }^{2}$

${ }^{1}$ Radiology, Mayo Clinic, Rochester MN USA, ${ }^{2}$ Cardiovascular Medicine, Mayo Clinic, Rochester MN USA

Keywords: carotid, atherosclerosis, plaque, CTA

Background: Using CTA to differentiate between components of carotid atherosclerotic lesions remains largely elusive. This study sought to validate a semi-automated software for CTA plaque analysis using histologic comparisons.

Materials and Methods: A retrospective review was performed of consecutive patients that underwent a carotid endarterectomy (CEA), with pre-procedural CTA imaging of the cervical arterial vasculature available for review. Images were evaluated using a commercially-available software package, which produced segmented analyses of intraplaque components (e.g. IPH, LRNC, and calcifications). On imaging, each component was assessed in terms of its 1) presence or absence, and 2) both volume and proportion of the total plaque volume (if present). On histological evaluation of CEA specimens, each component was evaluated as an estimated proportion of total plaque volume.

Results: Of 80 included patients, 30 (37.5\%) were female. The average age was 69.7 years $(\mathrm{SD}=9.1)$. Based on imaging, IPH was the smallest contributor to plaque composition ( $1.2 \%$ of volumes on average). Statistically significant linear associations were noted between the proportion of IPH, LRNC, and calcifications on histology and the volume of each component on imaging ( $\mathrm{p}$ values ranged from 0.0008 to 0.01 ). Area under curve (AUC) were poor for IPH and LRNC (0.59 and 0.61, respectively) and acceptable for calcifications (0.73).

Conclusion: Semi-automated analyses of CTA have limited diagnostic accuracy in the detection of IPH and LRNC in carotid artery plaques. However, volumetric imaging measurements of different components corresponded with histologic analysis.
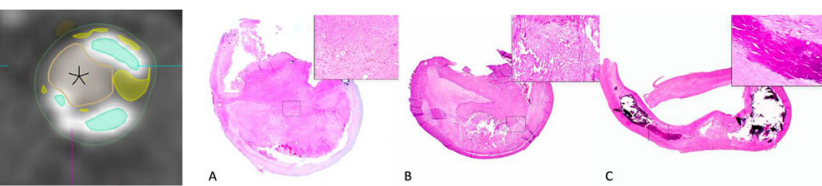

3-O2

INTRA VOXEL INCOHERENT MOTION (IVIM) 3T MRI FOR ORBITAL LESION CHARACTERIZATION

AUGUSTIN LECLER $^{1}$, Loic Duron ${ }^{1}$, Mathieu Zmuda ${ }^{2}$, Olivier Berges ${ }^{1}$, Julien Savatovsky ${ }^{1}$, Laure Fournier ${ }^{3}$

${ }^{1}$ Neuroradiology Department, A. Rothschild Foundation Hospital, Paris, France, 2 Ophthalmology Department, A. Rothschild Foundation Hospital, Paris, France, 3 Radiology Department, Pompidou Hospital, APHP, Paris, France

Keywords: IVIM, orbital neoplasms, MRI

Objectives: To determine the diagnostic accuracy of MRI Intravoxel incoherent motion (IVIM) when characterizing orbital lesions, which is challenging due to a wide range of locations and histologic types.

Methods: This IRB-approved prospective single-center study enrolled participants presenting with an orbital lesion undergoing a 3 Tesla MRI prior to surgery from December 2015 to July 2019. An IVIM sequence with $15 \mathrm{~b}$-values ranging from 0 to $2000 \mathrm{~s} / \mathrm{mm}^{2}$ was performed. Two neuroradiologists, blinded to clinical data, individually analyzed morphological MRIs. They drew one region of interest inside each orbital lesion, providing Apparent Diffusion Coefficient (ADC), True diffusion coefficient (D), Perfusion fraction (f) and Pseudodiffusion coefficient (D) values. T-test, Mann-Whitney U test and Receiver operating characteristic curve analyses were performed to discriminate between orbital lesions and to determine the diagnostic accuracy of the IVIM parameters.

Results: 156 participants ( 84 women and 72 men, mean age $54.4+/-17.5$ years) with 167 orbital lesions (98/167 [59\%] benign lesions including 54 orbital inflammations and 69/167 [41\%] malignant lesions including 32 lymphomas) were included in the study. 
$\mathrm{ADC}$ and $\mathrm{D}$ were significantly lower in malignant than in benign lesions: $0.8 \times 10-3 \mathrm{~mm} 2 / \mathrm{s}[0.45]$ versus $1.04 \times 10-3 \mathrm{~mm} 2 / \mathrm{s}[0.33], \mathrm{p}<0.001$ and $0.75 \times 10-3 \mathrm{~mm} 2 / \mathrm{s}[0.40]$ versus $0.98 \times 10-3 \mathrm{~mm} 2 / \mathrm{s}[0.42], \mathrm{p}<0.001$, respectively. $\mathrm{D}$ was significantly higher in malignant lesions than in benign ones: $12.8 \times 10-3 \mathrm{~mm} 2 / \mathrm{s}$ [20.17] versus $7.52 \times 10-3 \mathrm{~mm} 2 / \mathrm{s}$ [7.57], $\mathrm{p}=0.005$. Area Under Curve were of $0.73,0.74,0.72$ and 0.81 for $\mathrm{ADC}, \mathrm{D}, \mathrm{D}^{*}$ and a combination of $\mathrm{D}, \mathrm{f}$ and $\mathrm{D}^{*}$, respectively.

Conclusions: Our study showed that IVIM might help better characterize orbital lesions.

\section{3-O3}

A MAGNETIC RESONANCE IMAGING RADIOMICS SIGNATURE TO DISTINGUISH BENIGN FROM MALIGNANT ORBITAL LESIONS

LOİC DURON $^{1,2,4}$, Alexandre Heraud ${ }^{1}$, Frederique Charbonneau ${ }^{1}$, ${\text { Mathieu } \text { Zmuda }^{3}}^{3}$, Julien Savatovsky ${ }^{1,2}$, Laure Fournier ${ }^{4,5}$, Augustin Lecler $^{1,2}$

${ }^{1}$ Department of Neuroradiology, Foundation Adolphe de Rothschild Hospital, Paris, France, ${ }^{2}$ Department of Clinical Research, Foundation Adolphe de Rothschild Hospital, Paris, France, ${ }^{3}$ Department of Orbitopalpebral Surgery, Fondation Adolphe de Rothschild Hospital, Paris, France, ${ }^{4}$ Universite de Paris, PARCC, INSERM, F-75015 Paris, France, ${ }^{5}$ Radiology department, AP-HP, Hôpital europeen Georges Pompidou, F-75015, Paris, France

\section{Keywords: Orbit, Radiomics, Diagnosis}

Objectives: Distinguishing benign from malignant orbital lesions remains challenging both clinically and with imaging, leading to risky biopsies. The objective was to differentiate benign from malignant orbital lesions using radiomics on $3 \mathrm{~T}$ magnetic resonance imaging (MRI) examinations.

Materials \& Methods: This institutional review board-approved prospective single-center study enrolled consecutive patients presenting with an orbital lesion undergoing a $3 \mathrm{~T}$ MRI prior to surgery from December 2015 to July 2019. Radiomics features were extracted from 6 MRI sequences (T1-weighted images [WIs], DIXON-T2-WI, diffusion-WI, postcontrast DIXON-T1-WI) using the Pyradiomics software. Features were selected based on their intraobserver and interobserver reproducibility, nonredundancy, and with a sequential step forward feature selection method. Selected features were used to train and optimize a Random Forest algorithm on the training set $(75 \%)$ with 5-fold cross-validation. Performance metrics were computed on a held-out test set $(25 \%)$ with bootstrap $95 \%$ confidence intervals (95\% CIs). Five residents, 4 general radiologists, and 3 expert neuroradiologists were evaluated on their ability to visually distinguish benign from malignant lesions on the test set. Performance comparisons between reader groups and the model were performed using McNemar test. The impact of clinical and categorizable imaging data on algorithm performance was also assessed.

Results: A total of 200 patients (116 [58\%] women and 84 [42\%] men; mean age, $53.0 \pm 17.9$ years) with 126 of $200(63 \%)$ benign and 74 of 200 (37\%) malignant orbital lesions were included in the study. A total of 606 radiomics features were extracted. The best performing model on the training set was composed of 8 features including apparent diffusion coefficient mean value, maximum diameter on T1-WIs, and texture features. Area under the receiver operating characteristic curve, accuracy, sensitivity, and specificity on the test set were respectively $0.869(95 \%$ CI, 0.834-0.898), 0.840 (95\% CI, 0.806-0.874), 0.684 (95\% CI, 0.6150.751 ), and 0.935 (95\% CI, 0.905-0.961). The radiomics model outperformed all reader groups, including expert neuroradiologists $(\mathrm{P}<$
0.01). Adding clinical and categorizable imaging data did not significantly impact the algorithm performance $(\mathrm{P}=0.49)$.

Conclusions: An MRI radiomics signature is helpful in differentiating benign from malignant orbital lesions and may outperform expert radiologists.

ROC Curves and Reader Groups Performance

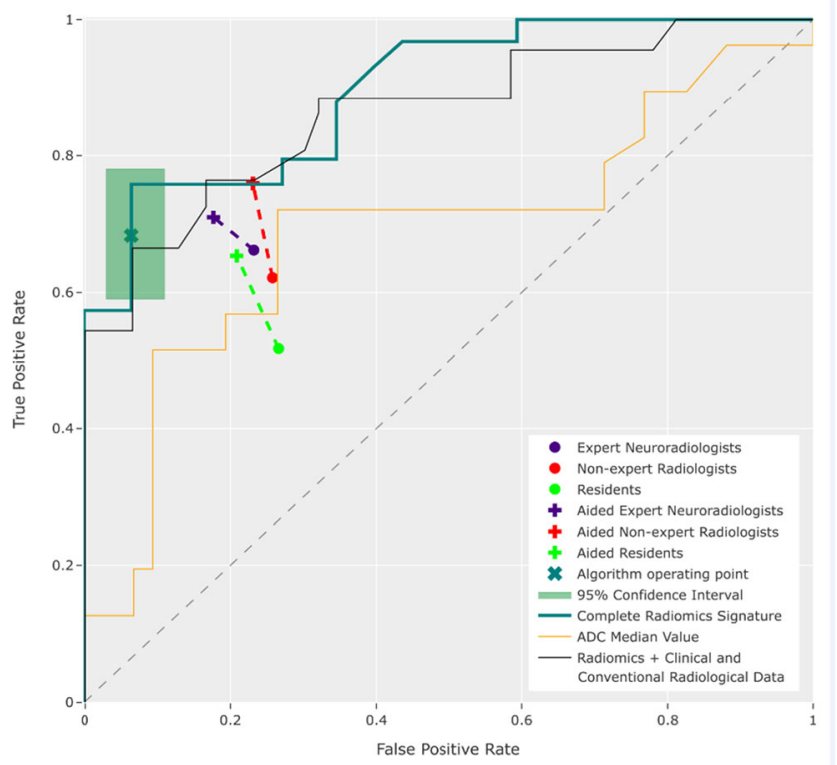

\section{PEDIATRIC}

\section{4-01}

A DIAGNOSTIC ALGORITHM FOR POSTERIOR FOSSA TUMORS IN CHILDREN: A VALIDATION STUDY

FELICE D'ARCO ${ }^{1}$, Cesar Augusto Pinheiro Ferreira Alves ${ }^{2}$, Ulrike Loebel $^{1}$, Kshitij Mankad ${ }^{1}$, Sara Teixeiras ${ }^{2}$, Fabricio Guimaraes Goncalves $^{2}$, Arastoo Vossough ${ }^{1}$

${ }^{1}$ Great Ormond Street Hospital, London, UK, ${ }^{2}$ Children Hospital of Philadelphia, Philadelphia USA

Keywords: medulloblastoma, posterior fossa tumors, ATRT, ependymoma

Background \& Purpose: Primary posterior fossa tumors comprise a large group of neoplasias with variable aggressiveness and short and long-term outcomes. This study aimed to validate the clinical usefulness of a radiologic decision flow chart based on previously published neuroradiologic knowledge for the diagnosis of posterior fossa tumors in children.

Materials \& Methods: A retrospective study was conducted (from January 2013 to October 2019) at 2 pediatric referral centers, Children's Hospital of Philadelphia, United States, and Great Ormond Street Hospital, United Kingdom. Inclusion criteria were younger than 18 years of age and histologically and molecularly confirmed posterior fossa tumors. Subjects with no available preoperative MR imaging and tumors located primarily in the brain stem were excluded. Imaging characteristics of the tumors were evaluated following a predesigned, step-by-step flow 
chart. Agreement between readers was tested with the Cohen k, and each diagnosis was analyzed for accuracy.

Results: A total of 148 cases were included, with a median age of 3.4 years (interquartile range, 2.1-6.1 years), and a male/female ratio of 1.24. The predesigned flow chart facilitated identification of pilocytic astrocytoma, ependymoma, and medulloblastoma sonic hedgehog tumors with high sensitivity and specificity. On the basis of the results, the flow chart was adjusted so that it would also be able to better discriminate atypical teratoid/rhabdoid tumors and medulloblastoma groups 3 or 4 (sensitivity $1 / 475 \%-79 \%$; specificity $1 / 492 \%-99 \%$ ). Moreover, our adjusted flow chart was useful in ruling out ependymoma, pilocytic astrocytomas, and medulloblastoma sonic hedgehog tumors.

Conclusions: The modified flow chart offers a structured tool to aid in the adjunct diagnosis of pediatric posterior fossa tumors. Our results also establish a useful starting point for prospective clinical studies and for the development of automated algorithms, which may provide precise and adequate diagnostic tools for these tumors in clinical practice.

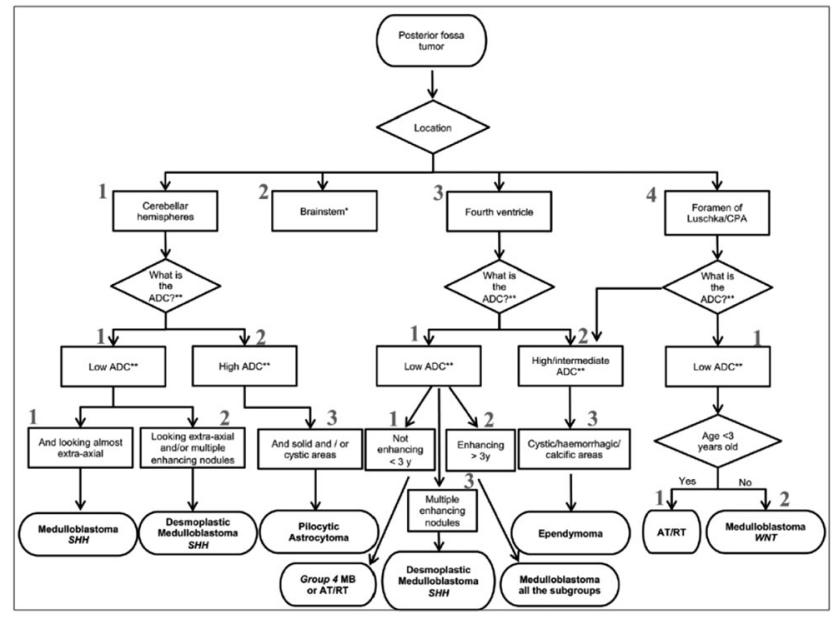
FIG 1. Predesigned radiologic flow chart created according to the literature before diagnostic accuracy analysis. The asterisk indicates brain
stem tumors excluded from the analysis Double asterisks indicate relative to gray matter. Modified with permission from D'Arco et al.".
4-02

NEUROIMAGING PATTERNS IN BACTERIAL MENINGITIS IN NEWBORNS

MARIANA PLANELLS ALDUVIN ${ }^{1}$, Marta Gomez- Chiari ${ }^{1}$, Monica Rebollo $^{1}$, Jordi Muchart ${ }^{1}$, Josep Munuera ${ }^{1}$

${ }^{1}$ Hospital Sant Joan de Deu, Barcelona, Spain

Keywords: meningitis, newborn, infection

Bacterial meningitis is the most common infection of the central nervous system in newborns. It is a life-threatening disease with a rapid instauration of symptoms which can include lethargy, poor feeding and irritability.

In neonates, the most common pathogens are Group B Streptococcus, E. coli, Listeria and Enterobacter. Some risk factors include antenatal chorioamnionitis, premature rupture of membranes, low birth weight, male gender, invasive monitoring, and need for resuscitation.

Diagnosis of bacterial meningitis is performed through clinical evaluation and CSF analysis. Imaging evaluation can be performed through cranial ultrasound, CT and MRI. Cranial ultrasound is usually the first imaging modality performed in critically ill patients at bedside. Ultrasound can be useful in the depiction of hydrocephalus, hyperechogenic sulci, subdural effusions and cerebral abscesses. CT is usually reserved for emergency settings due to ionizing radiation but it has the advantages of a faster acquisition and it is useful in the diagnosis of intracranial haemorrhage. MRI is the imaging modality of choice to exclude contraindications for lumbar puncture and to assess complications such as hydrocephalus, abscesses, subdural effusions, vascular complications such as infarcts, and meningitic labyrinthitis some of which can be related to certain pathogens. MRI findings can include hyperintense sulci and cisterns on fluid attenuation inversion recovery (FLAIR) sequences. Although contrastenhanced T1-weighted sequence and FLAIR are the most accurate sequences for detection of acute meningitis, at our institution we try to avoid administering contrast to neonates, for this reason we find very useful to perform diffusion weighted images in the axial and coronal planes. Susceptibility-weighted images (SWI), gradient echo, and T1-weighted images are highly sensitive for any associated hemorrhage.

The purpose of this review is to illustrate neuroimaging patterns that can correlate to some of these bacteria. A generalized bacterial meningitis can be found mostly in E. coli and group B Streptococcus, in which a cycle of arachnoiditis, vasculitis, vasogenic and cytotoxic oedema and ventriculitis can be observed. Cerebral hemorrhagic necrosis is more common in gram negative bacteria. Ischemic lesions can be observed in Group B Streptococcus. Subdural infection and abscesses are mostly associated with pneumococcal meningitis but also seen in streptococcal and staphylococcal meningitis. Ischemia as well as basal ganglia involvement has also been observed in streptococcal meningitis. Ventriculomegaly, ventriculitis, and DWI-restricted extra-axial collections are more commonly found in E. coli meningitis. Multiple large intraparenchymal abscesses can be seen as a complication in Serratia meningitis.

\section{SPINE}

\section{5-O1}

INTRAMEDULLARY TUMOURS OF THE CONUS MEDULLARIS

SOFIA BETTENCOURT $^{1}$, Bruno $\mathrm{Cunha}^{2}$, Pedro S. Brandao ${ }^{2}$, Mariana Baptista $^{2}$, Carla Conceicao ${ }^{2}$ 
${ }^{1}$ Neuroradiology Department, Hospital Sao Jose Lisboa, Lisbon, Portugal, ${ }^{2}$ Neuroradiology Department, Centro Hospitalar Universitario de Lisboa Central, Lisbon, Portugal

Keywords: spine, spinal tumours, MR imaging, conus medullaris, intramedullary tumour, renal cell carcinoma metastasis, pilocytic astrocytoma, high-grade glioma, embryonal tumour of the CNS, primary CNS melanoma

Introduction: Lesions affecting the conus medullaris can be divided according to their topographic location. They can be distinguished as arising extradurally, intradural-extramedullary or intramedullary. Intramedullary conus medullaris lesions include neoplasm, infection, inflammatory, congenital and vascular lesions. Conus medullaris tumours are responsible for $10 \%$ of all intramedullary spinal tumours. This region is mainly affected by tumours of glial cell type origin, with ependymoma being the most frequent one. However, other tumour types must be taken into consideration when evaluating this region.

The most frequent complaint of patients with intramedullary conus tumour is lumbar back pain, which may be non-specific initially, with later progression to a defined bilateral leg pain, typically accompanied by bladder and bowel dysfunction. Motor impairment may only present at advanced stages, with the initial symptoms being treated conservatively. Hence, in most cases there is a delay of several months before a radiological diagnosis is established.

Method: We retrospectively collected cases of intramedullary spinal tumours in the conus medullaris and provide a pictorial review depicting the key clinical and imaging findings that can help narrow the differential diagnosis.

Result: In our series we describe five different cases of intramedullary spinal tumours involving the conus medullaris, including a renal cell carcinoma metastasis, a pilocytic astrocytoma, a high-grade glioma, an embryonal tumour of the CNS NOS and a primary CNS melanoma.

Conclusion: Imaging and particularly MRI is a cornerstone in the diagnosis of intramedullary tumours of the conus medullaris, evaluating its morphologic and signal characteristics, enhancement pattern and tumour extension. Since prompt treatment is essential for preventing progression of neurological deficits and allow symptomatic relief, the knowledge of the clinical background and the distinct imaging characteristics of the tumours that may affect this region can help narrow the differential diagnosis.

\section{5-O2}

CT-GUIDED INTRATHECAL NUSINERSEN ADMINISTRATION IN ADULT SPINAL MUSCULAR ATROPHY PATIENTS WITH COMPLEX SPINAL ANATOMY

CATARINA MENDES PINTO ${ }^{1}$, Maria Joao Malaquias ${ }^{2}$, Vasco Sousa Abreu $^{1}$, Ricardo Cruz Martins ${ }^{1}$, Ana Paula Sousa ${ }^{2}$, Joao Xavier ${ }^{1}$

\footnotetext{
${ }^{1}$ Neuroradiology Department, Centro Hospitalar Universitario do Porto, Porto, Portugal, ${ }^{2}$ Neurology Department, Centro Hospitalar Universitario do Porto, Porto, Portugal
}

Keywords: CT-guided puncture, nusinersen, spinal muscular atrophy, intrathecal injection

Introduction: Spinal muscular atrophy (SMA) is a motor neuron disease, caused by genetic defects in the SMN complex. Recently, Nusinersen was approved as the first drug for the treatment of all forms of SMA, including adults. Intrathecal administration of Nusinersen in adult SMA patients is often complicated by scoliosis and previous spinal surgery with metal implantation, with CT-guided approach being a good alternative.

Methods: We reviewed all adult SMA patients followed in our university hospital between 2019 and 2020. Of those, 6 patients were eligible for intrathecal administration of Nusinersen. Two patients had complex spinal anatomy and a CT-guided intrathecal Nusinersen injection was performed.

Results: We performed the procedure in 2 patients with SMA type 3 (1 female, 1 male). At first intrathecal administration, patients were 29 and 36 years old. At baseline, the Hammersmith Functional Motor Scale Expanded (HFMSE) was 9/66 and 8/66, and the Revised Upper Limb Module (RULM) was 21/37 and 16/37 for each patient. Both cases had scoliosis and spinal surgery had been performed in one patient with an extensive posterior spinal fusion between D1 and S1.

The intrathecal administration of Nusinersen was performed with CTguided interlaminar lumbar puncture in the patient with scoliosis and with translaminar approach in the patient with the spinal fusion. Each patient completed all 4 sessions with loading dose (for a total of 8 CT-guided lumbar punctures performed), with a technical success of $100 \%(8 / 8$ intrathecal infusions). The mean radiation exposure per patient given by dose-length product (DLP) was $997.3 \mathrm{mGy} * \mathrm{~cm}$ for the patient with spinal fusion and $458.4 \mathrm{mGy} * \mathrm{~cm}$ for the patient with scoliosis. Procedure duration, and consequently the DLP, were highest at the first intervention. There was one adverse event with severe post-procedure headache.

Median follow-up from the first injection was $295 \pm 63$ days and the patients remained stable, with no further motor deterioration.

Discussion/Conclusion: With the presented cases we confirmed the safety of CT-guided Nusinersen injection in adults SMA patients. We demonstrate that Nusinersen can be successfully administrated even in patients with complex spinal anatomy, and we suggest the translaminar drilling technique as an alternative delivery route in patients with complete posterior spinal fusion. Therefore, intrathecal Nusinersen treatment should not be withheld from patients because of severe spinal deformities. DLP was high, and low-dose protocols should be pursued. Interventional neuroradiologists have an important role in planning and performing these procedures ensuring their safety and efficacy.

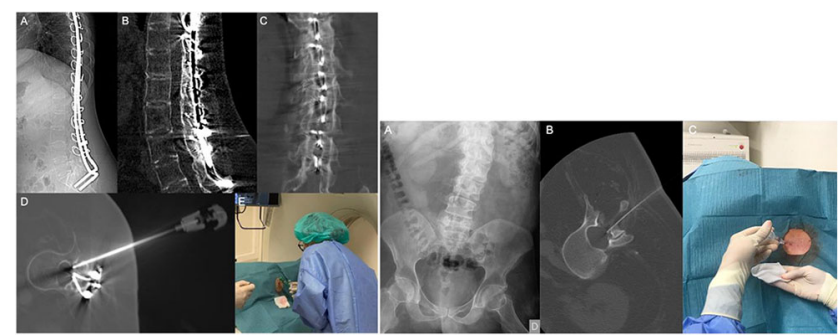

\section{5-O3}

EVOLUTION AND PROGNOSTIC VALUE OF INTERVERTEBRAL DISC T 2 -MAPPING VALUES AFTER OZONE CHEMONUCLEOLYSIS IN PATIENTS WITH LUMBAR DISC HERNIATION

FEDERICO BRUNO $^{1}$, Emanuele Tommasino ${ }^{1}$, Alessio Gagliardi ${ }^{1}$, Marco Varrassi ${ }^{1}$, Alessandra Splendiani ${ }^{1}$

${ }^{1}$ Department of Biotechnology and Applied Clinical Sciences, University of L'Aquila, L'Aquila, Italy

Keywords: ozone discolysis, lumbar disc heniation, T2 mapping MRI 
Purpose: To assess the validity of the MR T2-mapping sequence in estimating the modifications of the intervertebral disc (IVD) treated by $\mathrm{O} 2$ - $\mathrm{O} 3$ chemodiscolysis and its possible role in predicting clinical outcomes.

Methods: 40 patients with Low Back Pain (LBP) (22 males, 18 females; mean age 46.15 years) were enrolled for percutaneous CT-guided O2-O3 chemodiscolysis. Thirty-one sex- and age-matched patients, served as controls and were treated by CT-guided periradicular injections. All patients were assessed clinically trough the Visual Analogue Scale (VAS) and the Oswestry Disability Index (ODI), and radiologically, by using a 3Tscanner, for evaluating the intervertebral disc area (IDA) and the T2mapping values of the IVD before, at 1-month, and 6-months follow-up.

Results: The mean pre-treatment T2 relaxation time values were $38.80 \pm$ $4.51 \mathrm{~ms}, 44.05 \pm 0.91 \mathrm{~ms}$, and $45.45 \pm 14.11 \mathrm{~ms}$ for the anterior annulus fibrosus (aAF), nucleus pulposus (NP), and posterior annulus fibrosus (pAF), respectively, with a significant increase at the level of the NP ( p $<0,05)$ at the 1-month follow-up. The 6-months follow-up showed a reduction with normalization of intradiscal $\mathrm{T} 2$ relaxation time values showed a significant correlation of NP values with both the reduction of IDA $(0.81, p<0.001)$ and the improvement of clinical scores $(0.86$, $\mathrm{p}<0.001)$. In the control group, despite the clinical improvement, we did not find significant IVA reduction nor significant $\mathrm{T} 2$ values changes after treatment.

Conclusions: T2-mapping may be a useful indicator to predict disc chrinkage and the clinical response to CT-guided 02 - 03 injection.

\section{5-O4}

THE MODULAR APPROACH COMPLEX KYPHOPLASTY (MACK) PROCEDURE: PROOF OF CONCEPT AND RATIONALE

\section{GERARD DEIB $^{1}$, Taylor Rombold ${ }^{2}$, Doug Beall ${ }^{3}$}

${ }^{1}$ Neuroradiology, West Virginia University, Morgantown, USA, ${ }^{2}$ Cadelle Medical, Pennsylvania, USA, ${ }^{3}$ Interventional Musculoskeletal Radiology, Clinical Radiology of Oklahoma, Edmond, Oklahoma, USA

Keywords: Kyphoplasty, fracture, vertebral augmentation, pedicle screws

Introduction: The Modular Approach Complex Kyphoplasty (MACK) technique is a fluoroscopically guided, percutaneous, internal fixation technique which allows for treatment versatility across fracture types using an augmented kyphoplasty technique, which anchors an anterior vertebral implant (Spine Jack (SJ) Stryker Kalamazoo, MI)/cement complex with pedicle screws to the posterior vertebral elements. MACK achieves vertebral body (VB) reconstruction, augmentation, and stabilization in severe osteoporotic, traumatic and neoplastic fractures.

Methods: We present a proof of concept based on work done on an anatomic model. Spine Jack (SJ) Implants are placed in the ventral aspect of the vertebral body to achieve controlled anatomical height restoration, improvement in kyphosis and increased axial loading capacity before cement augmentation. Subsequently, percutaneous, fenestrated, pedicle screws are inserted. The fenestrated screws allow for additional cement administration and stabilization of the middle and posterior components with consequent bridging across all 3 columns. Screws with a modular tulip component are used which allows the operator to place the screws without the tulip(s). In the context of even more complex fractures or multiple fractures where posterior fixation may be required, the tulip(s) may be added and utilized for stent-screw and posterior rod fixation.
Results: The MACK technique builds on work done previously on the 'armed concrete' approach featuring the Stent-Screw Assisted Implant Technique (SAIF) as described by Cianfoni et al (1), and the PedicleJack procedure, created by Vexim (Balma, France) and described by Beall et al. The spine jack used in the MACK approach differs significantly from the Spine Stent (SS) (Depuy Synthes Raynham, MA) Implant (as used in the SAIF technique) in that it utilizes a mechanical rather than a hydraulic working system and has a self-locking deployment system through which extreme load forces concentrated on the device(s) result in an automatic block $(2,3)$. This mechanism restricts further expansion of the device, reducing the risk of vertebral endplate breakage. By reducing the superior endplate, a better functional recovery of the injured disc is facilitated.

Conclusion: This minimally-invasive procedure offers a viable alternative to invasive anterior and posterior column surgical options (such as corpectomy and/or pedicle screw and rod fixation) in situations previously unamenable to percutaneous intervention. Most notably, severe fractures with a high degree of fragmentation and collapse, may be amenable to this approach, as are fractures with large clefts, middle and posterior column involvement and vertebrae with extensive marrow and tumor infiltration.

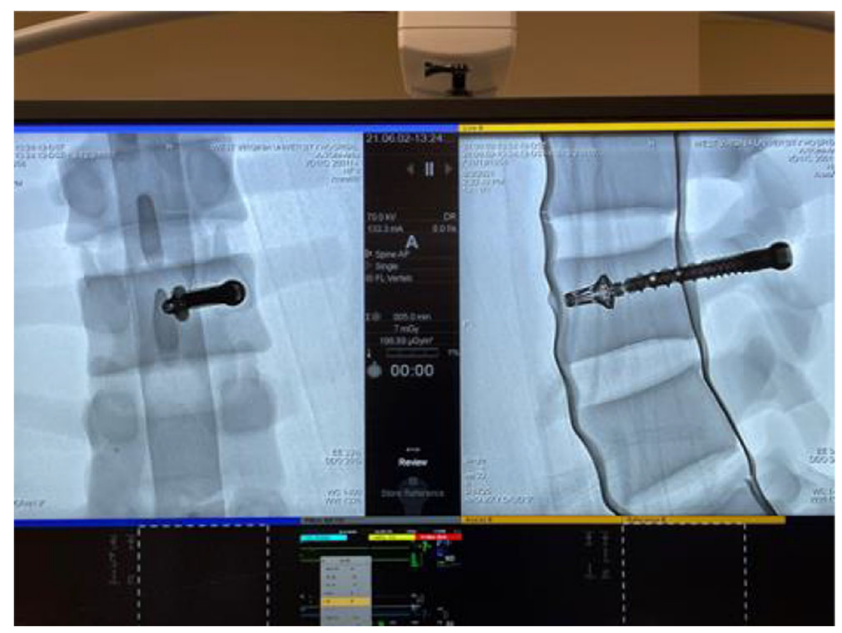

E-POSTERS (original and unedited texts as received by the authors)

\section{DIAGNOSTIC}

1-P1

MODERN NEURONAVIGATION POSSIBILITIES FOR IDENTIFICATION OF THE BOUNDARIES OF GLIOMAS

RYNDA ARTEMII YURIEVICH $^{1}$, Victor Emelijanovich Olyushin ${ }^{1}$, Dmitry Michailovich Rostovtsev $^{1}$, Yliay Michaiylovna Zabrodskaya ${ }^{1}$, Harry Vazgenovich Papayan ${ }^{1}$

${ }^{1}$ Polenov A.L. Russian Research Institute of Neurosurgery - V.A. Almazov National Medical Research Center, Ministry of Health of Russia, St. Petersburg, RUSSIA

Keywords: PET, MRI, glioma, specificity, sensitivity, fluorescence navigation

Objective: To evaluate the effectiveness and feasibility of using fluorescence navigation with chlorin E6 in the surgery of gliomas of various degrees of malignancy. 
Materials \& Methods: The study included 30 patients with glial tumors of varying degrees of malignancy according to Grade (II-IV). For visual fluorescence studies, equipment was used in the form of a Leica OHS surgical microscope, a D-Light AF System installation by Karl Storz (Germany). For histological study of biopsy samples, hematoxylineosin staining and immunohistochemical studies were adopted.

Results: In 30 patients with fluorescence navigation, the contrast was assessed on preoperative MRI images in T1 mode. A comparative analysis of visible fluorescence with the data of preoperative MRI with contrast (T1 mode) showed a direct correlation ( $p>0.05)$ from the accumulation of contrast on MRI and the degree of glioma anaplasia. The zone of tumor necrosis corresponded to the absence of contrast accumulation on MRI and the absence of chlorine e6 fluorescence. The zone of the compact part of the tumor corresponded to the maximum level of contrast accumulation on MRI and the maximum fluorescence of chlorine e6 $(+++)$. The zone of cellular infiltration corresponded to a non-intense, uneven accumulation of contrast on MRI and less intense (++ and +) fluorescence. Benign gliomas were characterized by a more uniform and non-intensive accumulation of contrast on MRI, an MRI picture closer to the normal structure of the gyri, and homogeneous moderate $(++)$ or weak (+) fluorescence.

Comparative analysis of PET with methionine and chlorin e 6 conditioned fluorescence intensity of chlorine e $6(\mathrm{p}<0,05)$. A higher accumulation index (1.7-3.9) and brighter fluorescence of chlorine e6 (+++) were noted in glioblastoma (Grade IV). A low accumulation index (0.7) and very weak fluorescence $(+)$ were noted in Grade II tumors. The sensitivity of the method of fluorescence diagnostics with chlorin E6 for Grade II gliomas was $72.2 \%$, for Grade III gliomas $-83.8 \%$, for Grade IV $87.7 \%$. The specificity of the method was $60 \%$ for Grade II gliomas, $66.7 \%$ for Grade III gliomas, and $85.2 \%$ for Grade IV.

Conclusion: The use of a certain system of fluorescence imaging allowed resection of glial brain tumors using chlorin E6, and the intensity of tumor fluorescence correlated with the degree of glioma malignancy. These results indicate that chlorin E6 is an effective photosensitizer for intraoperative fluorescence diagnostics in glioma surgery.

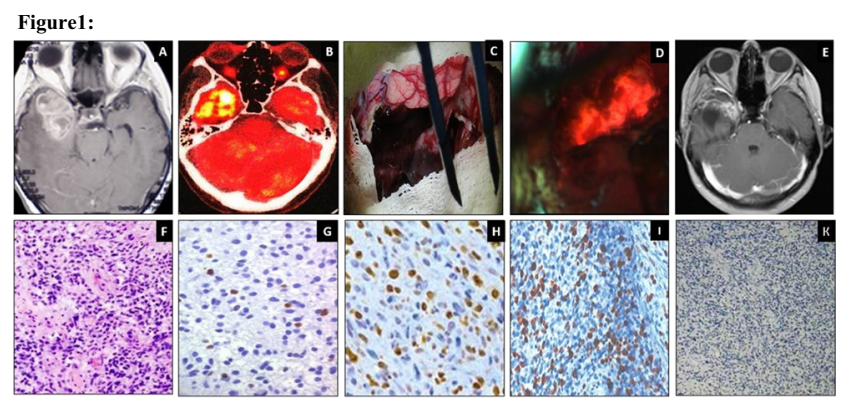

Figure 2:

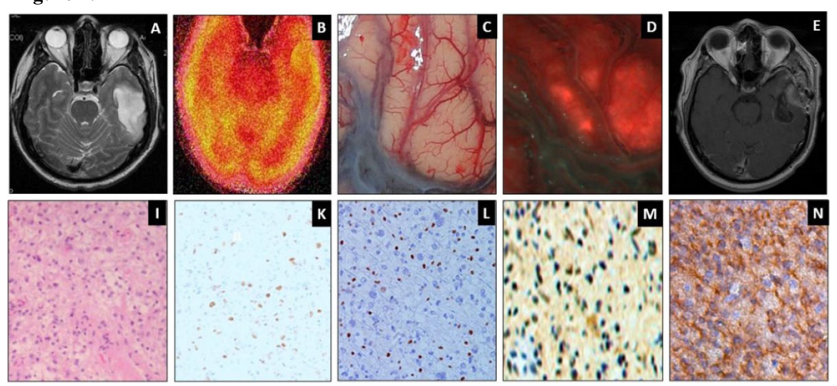

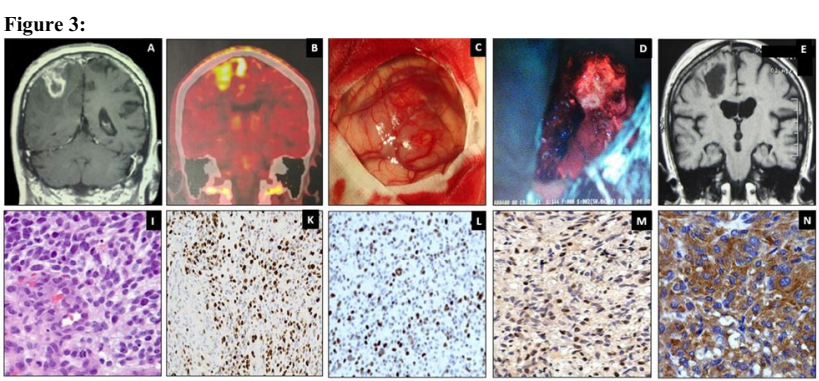

\section{1-P2}

PERFUSION IMAGING OF GLIOMAS USING ASL-LABELING

$\underline{\text { RYNDA ARTEMII YURIEVICH }}^{1}$, Victor Emelijanovich Olyushin $^{1}$, Dmitry Michailovich Rostovtsev

${ }^{1}$ Polenov A.L. Russian Research Institute of Neurosurgery - V.A. Almazov National Medical Research Center, Ministry of Health of Russia, St. Petersburg, RUSSIA

Keywords: neurooncology, glioma, ASL-LABELING

Objective: To evaluate the relationship between the measurement of blood flow in a tumor based on perfusion imaging using spin labeling of arteries (ASL-PI) and histopathological findings in gliomas of various grades of malignancy.

Methods: This study included 20 patients (10 high grade gliomas and 10 low grade gliomas). All patients received a multiphase multislice ASL MRI pulse sequence. As an index of tumor perfusion, the relative signal intensity (SI) of each tumor (\% signal intensity) was defined as a percentage of maximum tumor SI per mean SI in normal gray matter of the brain on ASL-PI. Relative vascular attenuation was defined as the total area of microvessels over the entire tissue area in histopathological specimens. The indicators of MIB1 gliomas were also calculated. Differences in percentage signal intensity were compared between different histopathological types and between high and low grade gliomas. The correlation between ASL perfusion and the degree of glioma anaplasia was assessed using Spearman's correlation analysis.

Results: There was a statistically significant difference between low and high grade tumors. Correlation analysis revealed significant positive correlations between tumor grade and ASL-PI perfusion $(\mathrm{p}<0.002)$.

Conclusion: ASL-PI is an effective imaging technique for non-invasive assessment of tumor perfusion and can predict histopathological vascular density of glial brain tumors and may be useful in distinguishing between high and low grade gliomas. 


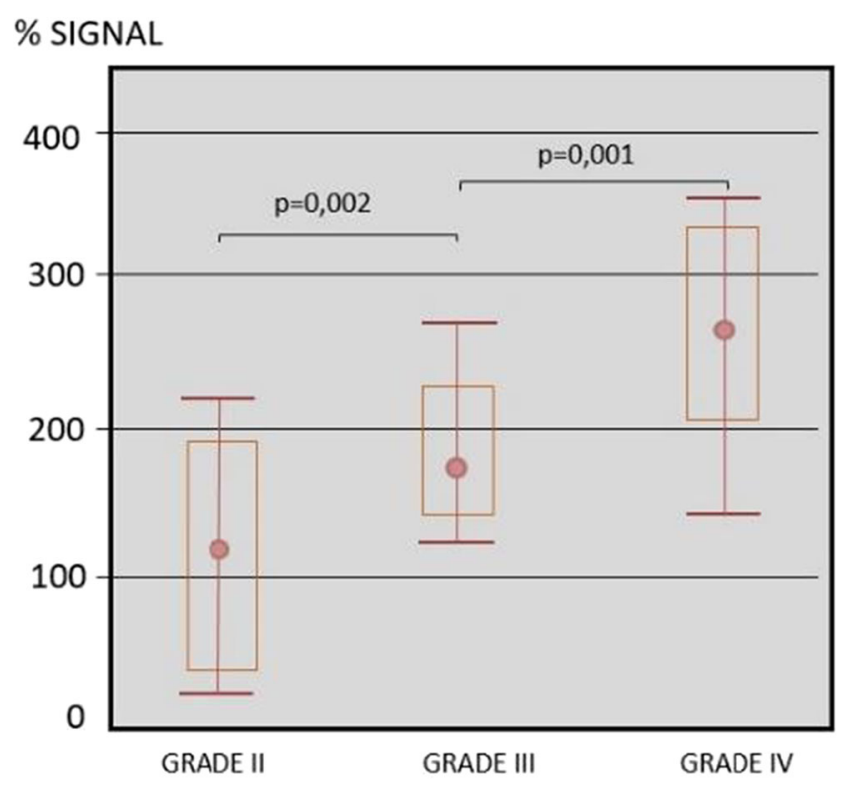

1-P3

RADIOLOGICAL-ANATOMICAL DISTRIBUTION PATTERNS OF JAKOB-CREUTZFELDT DISEASE IN RELATION TO CORTICAL SYSTEMS - INVOLVEMENT OF THE CORTEX WITH REGARD TO PHYLOGENESIS

FELIX GUNZER $^{1}$, Eva Hassler ${ }^{1}$, Gernot Reishofer ${ }^{2}$, Fritz Studencnik ${ }^{2}$, Hannes Deutschmann ${ }^{1}$, Josef Simbrunner ${ }^{1}$

${ }^{1}$ Division of Neuroradiology, Vascular and Interventional Radiology, Department of Radiology, Medical University Graz, Austria, Departement of Radiology, Medical University Graz, Austria

Keywords: Jakob-Creutzfeldt disease, brain segmentation, dual-origin concept, automatic labelling, phylogenesis, brodmann-areas labelling, association cortices

Introduction: Prion diseases are a group of diseases that affect humans and animals, usually present with rapidly progressive symptoms and are always fatal. This study investigates the radiological distribution patterns of cortical lesions on MRI in patients with Jakob-Creutzfeldt disease. This study aims to correlate these lesions with functional cortical systems based on the dual-origin concept, the cortex's phylogenesis and areas of long-range fibre tracts to assess whether Jakob-Creutzfeldt disease lesions on MRI and the respective clinical course can be expressed through one of these concepts, which has not been investigated before.

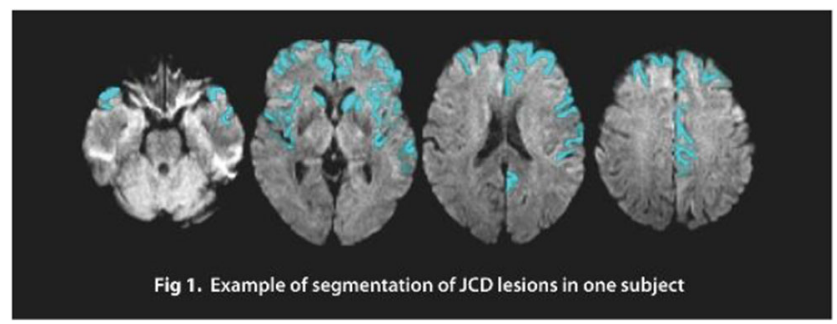

Methods: Ten patients with the diagnosis Jakob-Creutzfeldt disease were retrospectively selected, and the corresponding MRI images (DWI, ADC, FLAIR, T2) were extracted. The DWI scans were normalised using
SPM12 and registered in MNI space. The brain lesions were segmented and then automatically assigned to neuroanatomical areas using a selfwritten program code. The Talairach atlas and the AAL atlas were used to assign the respective MRI voxels and was followed by statistical analyses via RStudio.

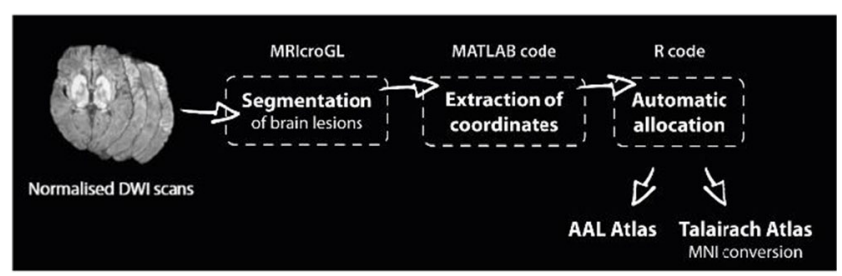

Results: Analysis of the labelled Jakob-Creutzfeldt disease lesions revealed no difference in distribution between the left and right hemisphere of the brain. The isocortex is affected the most, however, considering their volumes, the mesocortex showed almost two times more lesions. $81.7 \%$ of lesions were located in association cortices connected via longrange fibre tracts. There was no difference in distribution of lesions between the auditory, motor, somatosensory or visual system of the cortex. Paralimbic areas were most dominantly affected by Jakob-Creutzfeldt disease.

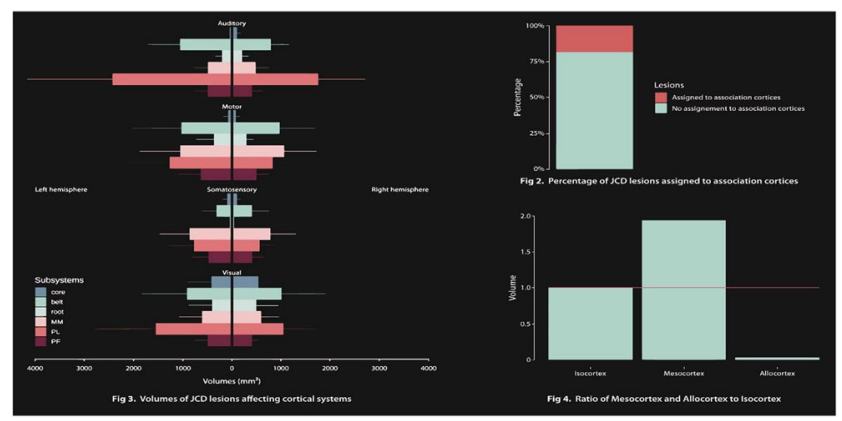

Discussion \& Conclusion: Paralimbic areas which are partly portrayed by the mesocortex seem to be relatively more often involved than other cortical areas. This might be because prions show different levels of affection to evolutionary different configurations of upper cortical neurons in the cortex. Hypotheses on the axonal transport of prions and the involvement of mainly neocortical structures could be consolidated. Clinical symptoms and functional cortical systems do not seem to correlate. Based on our results and visual confirmation through ADC maps, the assumption that Jakob-Creutzfeldt disease spares the Rolandi cortex should be reconsidered.

\section{References:}

1. Fragoso DC et al. Imaging of Creutzfeldt-Jakob Disease: Imaging Patterns and Their Differential Diagnosis. RadioGraphics. 2017 Jan;37(1):234-57.

2. Jansen GH et al. Distribution of Cellular Prion Protein in Normal Human Cerebral Cortex - Does It Have Relevance to CreutzfeldtJakob Disease? Clin Chem Lab Med. 2001 Jan 25;39(4).

1-P4

NEUROIMAGING FINDINGS IN COVID-19: A TERTIARY CARE CENTER EXPERIENCE 
SATHISH KUMAR DUNDAMADAPPA ${ }^{1}$, Lucinda Chiu ${ }^{1}$, Saurabh Rohatgi ${ }^{1}$, Andrew Chen ${ }^{1}$, Matthew Kona ${ }^{2}$, David $\mathrm{Choi}^{1}$

${ }^{1}$ Umass Memorial Medical Center-University of Massachusetts Medical School Worcester, MA, USA, ${ }^{2}$ Hunter Holmes McGuire VA Medical Center, 1201 Broad Rock Blvd, Richmond, VA, USA

Keywords: neuroimaging, COVID-19, mulitsystem involvement

Background: COVID-19 which is caused by SARS-CoV-2 is a novel disease which has rapidly spread to become a pandemic. The disease is known to have multiorgan involvement including the nervous system.

Purpose: This is a retrospective analysis of neuroimaging findings in admitted COVID-19 patients at our institution.

Materials \& Methods: This is a retrospective study of COVID-19 patients admitted to a tertiary care center between $2 / 1 / 2020$ and 5/25/202 Inclusion criteria were: (1) tested positive on a laboratory-based test for COVID-19; (2) had cross-sectional neuroimaging (CT and MRI of head, neck, and/or spine) following diagnosis of COVID-19/while COVID-19 positive; (3) showed acute findings on neuroimaging. Indications for imaging, patient demographics and acute neuroimaging findings in these patients were further analyzed.

Results: Seventy of 953 admitted COVID-19 patients had neuroimaging during study period. Most of the indications for neuroimaging belonged to either altered mentation category $(41 \%)$ or stroke like symptoms (24\%), with other less common indications included headache and seizures. Sixteen of these patients had acute neuroimaging findings that were probably caused by COVID-19. Most of these findings were either ischemic in nature $(56 \%)$ or hemorrhagic $(38 \%)$.

Conclusion: Neurological involvement by COVID-19 is increasingly recognized and reported. Patients in our study cohort predominantly had ischemic and hemorrhagic findings. Further long-term studies are needed to ascertain the neurological sequela and patterns of spread of SARS-CoV-2.

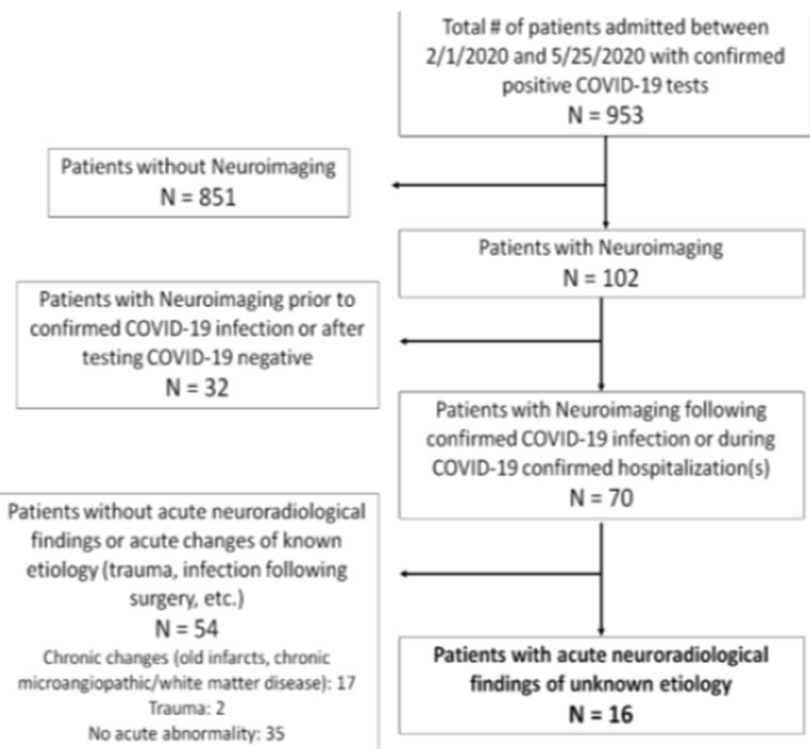

\begin{tabular}{|ll|}
\hline Characteristic & No. (\%) of patients \\
Gender & $44(62 \%)$ \\
Male & $26(38 \%)$ \\
Female & \\
Age group (year) & $2(3 \%)$ \\
$31-40 \mid$ & $7(10 \%)$ \\
$41-50$ & $8(11 \%)$ \\
$51-60$ & $19(27 \%)$ \\
$61-70$ & $21(30 \%)$ \\
$71-80$ & $10(14 \%)$ \\
$81-90$ & $3(4 \%)$ \\
$91-100$ & \\
Indication for Imaging & $32(41 \%)$ \\
$\begin{array}{l}\text { Altered mentation (altered mental status, } \\
\text { encephalopathy) }\end{array}$ & $17(24 \%)$ \\
Stroke-like symptoms (stroke, neurological \\
deficit, TIA) \\
Headache \\
Seizures \\
$\begin{array}{l}\text { Hemorrhage (concern for or known) } \\
\text { Miscellaneous (dysphagia, lethargy, } \\
\text { dizziness) }\end{array}$ \\
\hline
\end{tabular}

1-P5

INVESTIGATING MICROSTRUCTURAL CHANGES IN WHITE MATTER IN MULTIPLE SCLEROSIS: A SYSTEMATIC REVIEW AND INDIVIDUAL PARTICIPANT DATA META-ANALYSIS OF NEURITE ORIENTATION DISPERSION AND DENSITY IMAGING

ABDULMAJEED ALOTAIBI $^{1,2}$, Anna Podlasek $^{2}$, Amjad Altokhis $^{2}$, Rob Dineen $^{2}$, Cris S. Constantinescu ${ }^{2}$

${ }^{1}$ King Saud University for Health Sciences Riyadh, Saudi Arabia, ${ }^{2}$ University of Nottingham, Nottingham, UK

Keywords: NODDI, Multiple Sclerosis, Neurite density, Orientation dispersion, Multishell diffusion, MRI, Neuroaxonal pathology

Multiple Sclerosis (MS) is characterised by widespread damage of the central nervous system that includes demyelinating white matter (WM) lesions and alterations in normal-appearing white matter (NAWM). Neurite Orientation Dispersion and Density Imaging (NODDI) has been proposed to overcome some of the diffusion tensor imaging (DTI) limitations, providing a more precise characterization of WM microstructure. NODDI maps can be calculated for neurite density index (NDI), orientation dispersion index (ODI), which explicitly estimate the orientation dispersion and neurite density, all of which contribute to conventional DTI parameters. We conducted a systematic review and meta-analysis of studies using NODDI metrics to assess brain microstructural changes and neuroaxonal pathology in WM lesions and NAWM in patients with MS. Four electronic databases were searched and three reviewers conducted the search strategy, study screening/identification, study eligibility/ inclusion and quality assessment independently and blindly. The extent of between-study heterogeneity was assessed with the I 2 statistic. Study heterogeneity I2 values $>50 \%$ were considered substantial and $>75 \%$ was deemed considerable heterogeneity. Funnel plots and Egger's tests were used to assess publication bias for the primary outcome. P-values were 
two-tailed with values $<0.05$ considered statistically significant. All analyses were implemented using JASP 0.14.1.0 and Review Manager 5.4.1 software.

Neurite density (NDI) in WM lesions and NAWM were significantly reduced compared to healthy WM. The standardized mean difference was $-3.08(95 \% \mathrm{CI}-4.22$ to $(-1.95)), \mathrm{p}=<0.00001$ and $-0.70(95 \% \mathrm{CI}-$ 0.99 to $(-0.40)$ ), $\mathrm{p}=<0.00001$. It is considerable (WM lesions) and moderate (NAWM) heterogeneity between studies $\mathrm{I} 2=88 \%$ (WM lesions) and $\mathrm{I} 2=35 \%$ (NAWM). The Eggers test was $\mathrm{z}=2.135, \mathrm{p}=0.033$ which suggests potential publication bias for WM lesions. However, for NAWM, the Eggers test was $\mathrm{z}=-0.377, \mathrm{p}=0.706$, which suggests no publication bias. Orientation dispersion (ODI) in MS WM lesions and NAWM among 194 MS patients and 100 healthy control was not significant. The standardized mean difference was -0.44 (95\% CI -1.60 to $(-0.71)$ ), $\mathrm{p}=0.45$ (WM lesions) and was -0.46 (95\%CI -2.07 to $(-1.15)), \mathrm{p}=0.58$ (NAWM). There is considerable heterogeneity between studies $\mathrm{I} 2=95 \%$ (WM lesions) and $\mathrm{I} 2=89 \%$ (NAWM).

The findings have confirmed that NDI is significantly reduced in both MS lesions and NAWM compared to WM from healthy participants. We were unable to demonstrate differences in ODI in MS lesions and NAWM compared to WM from healthy participants but have identified that heterogeneity in studies may limit the meta-analysis. Further analysis of the use of the NODDI approach in MS for the characterisation of disease-related ultrastructural changes is justified.

\section{1-P6 \\ HIGH-RESOLUTION ISOTROPIC 3D DYNAMIC MR IMAGING OF THE PITUITARY GLAND USING COMPRESSED SENSE}

\section{KAZUHIRO TSUCHIYA ${ }^{1}$, Sayuki Hiraoka ${ }^{2}$, Hidekatsu Tateishi ${ }^{2}$}

${ }^{1}$ Department of Radiology, Saitama Medical Center, Saitama Medical

Keywords: MRI, pituitary gland, microadenoma, dynamic scan

Purpose: To evaluate the clinical feasibility of high-spatial-resolution isotropic three-dimensional (3D) dynamic MR imaging using compressed SENSE at 3T for the diagnosis of pituitary lesions.

Methods: We applied the 3D dynamic scan to 11 patients ( 9 females and 2 males, mean 55 years) with suspected pituitary or parapituitary lesion (2 with macroadenoma, 4 with microadenoma, 3 with Rathke's cleft cyst, 1 with parasellar meningioma, and 1 with cavernous sinus granuloma). All patients underwent sagittal and coronal precontrast 2D spin-echo (SE) images. High-spatial-resolution isotropic 3D dynamic MR imaging was performed on a 3-T imager in the coronal plane using a T1-weighted turbo SE sequence in combination with compressed SENSE (CSSENSE factor, 2.3) during a bolus injection of contrast material. The voxel size was $1.15 \times 1.15 \times 1.15 \mathrm{~mm}$. We acquired six phases in a total scanning time of $120 \mathrm{sec}(20 \mathrm{sec} \times 6)$. In addition to coronal source images, we generated sagittal and axial reformatted images. Thereafter, sagittal and coronal postcontrast T1-weighted 2D SE images were obtained. We assessed the tumor detectability of precontrast and postcontrast 2D SE MR images and 3D dynamic images based on the final diagnoses (surgery, 3; clinical diagnosis and follow up, 7).

Results: In all patients, we could obtain dynamic scan in the 3 planes with good contrast. Precontrast and postcontrast 2D SE images and dynamic $3 \mathrm{D}$ images visualized 8, 8, 11 lesions, respectively.

Discussion \& Conclusion: As this 3D dynamic scan generates highresolution isotropic images in three planes in one session, it can be a useful adjunct to the diagnosis of pituitary lesions, possibly replacing conventional 2D dynamic scan.

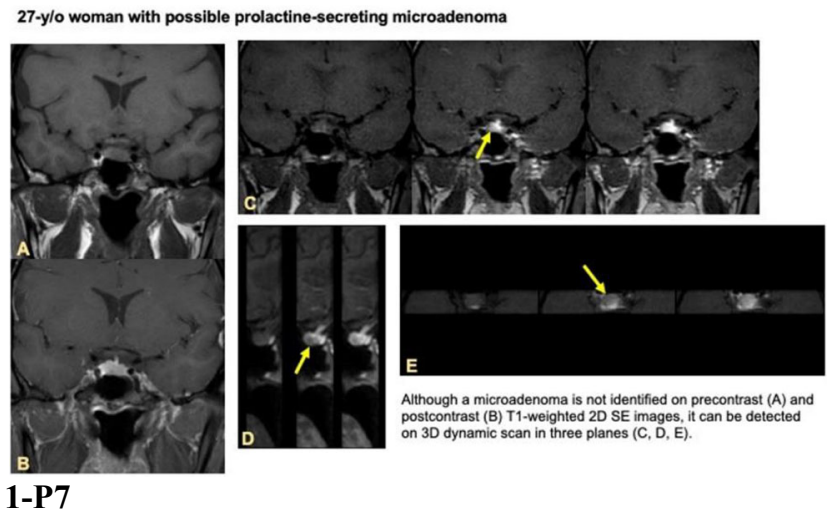

\section{1-P7}

CLINICAL EFFICACY OF REDUCED DOSE GADOBUTROL VS STANDARD-DOSE GADOTERATE IN CONTRAST-ENHANCED CNS IMAGING: AN INTERNATIONAL, MULTICENTER, PROSPECTIVE CROSS-OVER TRIAL (LEADER-75)

Benjamin P. Liu ${ }^{1}$, Martin Rosenberg ${ }^{2}$, Pollice Saverio ${ }^{3}$, Young Cheol Weon $^{4}$, Soenke Peters ${ }^{5}$, Francois-Daniel Ardellier ${ }^{6}$, Annette Boeckenhoff $^{7}, \underline{\text { JAN ENDRIKAT }}^{8,9}$

${ }^{1}$ Division of Neuroradiology, Departments of Radiology and Radiation Oncology, Feinberg School of Medicine of Northwestern University, Northwestern Memorial Hospital, Chicago, Illinois, USA, ${ }^{2}$ Bayer Pharmaceuticals, Radiology, Whippany, New Jersey, USA, ${ }^{3}$ Ospedale "L. Bonomo" - Andria, Viale Istria 1, Italy, ${ }^{4}$ Department of Radiology, Ulsan University Hospital, University of Ulsan, Korea, ${ }^{5}$ Department of Radiology and Neuroradiology, University Hospital of SchleswigHolstein, Campus Kiel, Arnold-Heller-Straße 3; D-24105 Kiel, Germany, ${ }^{6}$ Hôpitaux Universitaires de Strasbourg - Hôpital de Hautepierre, Avenue Moliere, Strasbourg 67200, France, ${ }^{7}$ Bayer AG, Statistics and Data Insights, D-42113 Wuppertal, Germany, ${ }^{8}$ Bayer AG, Radiology, D-13353 Berlin, Germany, ${ }^{9}$ University Medical School of Saarland, Dept of Gynecology, Obstetrics and Reproductive Medicine, D-66421 Homburg/Saar, Germany

\section{Keywords: Gadobutrol, Gadoterate, Dose, MRI, CNS}

Introduction: A dose reduction for a high-relaxivity gadolinium-based contrast agent (GBCA) is a desirable goal, especially for subjects who require multiple contrast-enhanced MRIs. The objective of this study was to demonstrate that the efficacy of a 25\%-reduced dose of gadobutrol (rdgadobutrol) is non-inferior to a standard-dose of gadoterate (sdgadoterate) for contrast-enhanced CNS MR imaging.

Methods: This international, prospective, multicenter, open-label, crossover trial recruited adult subjects with CNS pathologies. All had contrastenhanced magnetic resonance imaging (CE-MRI) with sd-gadoterate $(0.1 \mathrm{mmol} / \mathrm{kg}$ body weight [b.w.]) followed by a CE-MRI with rdgadobutrol $(0.075 \mathrm{mmol} / \mathrm{kg}$ b.w.) within two weeks. The primary efficacy variables (contrast enhancement, border delineation, internal morphology) were scored by three blinded readers using an ordinal scale with the primary analysis based on calculated 'average reader' results. The primary objective was to show non-inferiority of rd-gadobutrol to sd-gadoterate for all three primary variables. Non-inferiority was defined as rdgadobutrol minus unenhanced imaging achieving at least $80 \%$ of the difference of sd-gadoterate minus unenhanced imaging. An additional post-hoc clinically important analysis was added directly comparing the 
primary visualization variables for the combined (unenhanced plus enhanced) image sets.

Secondary efficacy variables included number of lesions detected, diagnostic performance, reader preference, diagnostic confidence, quantitative enhancement, and safety.

Results: All efficacy analyses included 141 subjects. The efficacy improvement of rd-gadobutrol over unenhanced imaging was non-inferior to the improvement of sd-gadoterate over unenhanced imaging using 20\% noninferiority margins and one-sided $(\alpha=0.025)$ tests for the average reader for all 3 visualization parameters (lesion contrast enhancement $-p<.0001$, border delineation $-\mathrm{p}=.0151$, and internal morphology $-\mathrm{p}=.0100$ ). The means of the average reader for the primary visualization variables of combined rd-gadobutrol $(2.979,3.099,2.484)$ and combined sd-gadoterate $(3.007,3.096,2.503)$ differed by less than $1 \%$ and are statistically equivalent using a $5 \%$ margin. The number of lesions detected by the average reader for rd-gadobutrol (301) and sd-gadoterate (291) were similar.

Discussion: The study results demonstrate that a $25 \%$ reduced dose of gadobutrol provided equivalent efficacy to a full dose of gadoterate for CNS imaging. Although no change in the adverse event profile for gadobutrol is expected with this modest dose reduction, providing radiologists the ability to reduce the gadolinium dose, especially in subjects who require multiple contrast-enhanced CNS procedures, is considered beneficial.

Conclusion: A $25 \%$-reduced dose of gadobutrol $(0.075 \mathrm{mmol} / \mathrm{kg}$ b.w. was equivalent to sd-gadoterate $(0.1 \mathrm{mmol} / \mathrm{kg}$ b.w.) for all 3 primary variables and noninferior when comparing the improvement over unenhanced imaging.

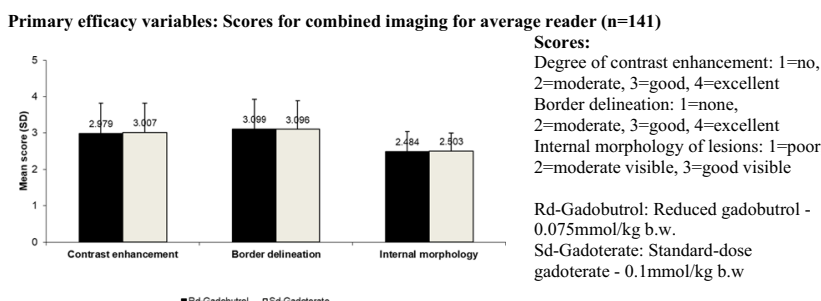

1-P8

INVESTIGATING BRAIN MICROSTRUCTURAL ALTERATIONS IN TYPE 1 AND TYPE 2 DIABETES USING DIFFUSION TENSOR IMAGING: A SYSTEMATIC REVIEW

ABDULMAJEED ALOTAIBI ${ }^{1,2}$, Christopher Tench $^{1}$, Rebecca Stevenson $^{1}$, Ghadah Felmban ${ }^{1,2}$, Amjad Altokhis ${ }^{1,3}$, Ali Aldhebaib ${ }^{2}$, Rob A. Dineen ${ }^{1,4}$, Cris S. Constantinescu ${ }^{1}$

${ }^{1}$ Division of Clinical Neuroscience, Nottingham University Hospitals NHS Trust, School of Medicine, University of Nottingham, Nottingham NG7 2UH, UK, ${ }^{2}$ School of Applied Medical Sciences, King Saud bin Abdul-Aziz University for Health Sciences, Riyadh 14611, Saudi Arabia, ${ }^{3}$ School of Health and Rehabilitation Sciences, Princess Nourah bint Abdulrahman University, Riyadh 11564, Saudi Arabia, ${ }^{4}$ NIHR Nottingham Biomedical Research Centre, Nottingham NG1 5DU, UK

Keywords: diabetes, DTI, MRI, Microstructural changes, Cognitive impairments, Diffusion imaging, type 1 diabetes, type 2 diabetes

Type 1 and type 2 diabetes mellitus have an impact on the microstructural environment and cognitive functions of the brain due to its microvascular/ macrovascular complications. Conventional Magnetic Resonance Imaging (MRI) techniques can allow detection of brain volume reduction in people with diabetes. However, conventional MRI is insufficiently sensitive to quantify microstructural changes. Diffusion Tensor Imaging (DTI) has been used as a sensitive MRI-based technique for quantifying and assessing brain microstructural abnormalities in patients with diabetes. This systematic review aims to summarise the original research literature using DTI to quantify microstructural alterations in diabetes and the relation of such changes to cognitive status and metabolic profile. A total of thirty-eight published studies that demonstrate the impact of diabetes mellitus on brain microstructure using DTI are included, and these demonstrate that both type 1 diabetes mellitus and type 2 diabetes mellitus may affect cognitive abilities due to the alterations in brain microstructures.

\section{1-P9}

COMPARISON OF NORMAL APPEARING WHITE MATTER RADIOMICS FEATURES, BASED ON PERIVASCULAR SPACE SCORES

ONURAL OZTURK $^{1}$, Gur Akansel $^{2}$, Sibel Balci $^{3}$, Seda Ozturk $^{4}$

${ }^{1}$ Department of Radiology, Gediz Public Hospital, Kutahya, Turkey, ${ }^{2}$ Department of Radiology, Kocaeli University, Kocaeli, Turkey, ${ }^{3}$ Department of Biostatistics and Medical Informatics, Kocaeli University, Kocaeli Turkey, ${ }^{4}$ Department of Neurology, Kocaeli University, Kocaeli, Turkey

Keywords: Radiomics, Virchow-Robin Space, Normal appearing white matter

Introduction: Perivascular space (PVS) is located around small arterioles or venules in brain. Clinical importance of PVS is disputed. "Radiomics" is quantitative data series of digital images in pixel-based approaches. The aim of our study is to investigate Radiomics features of normal appearing white matter (NAWM) in subjects with different PVS scores to display alterations not distinguishable with bare eye.

Methods: Adult patients underwent cranial MRI in a month period were screened. Upon applying exclusion criteria 160 patients were evaluated. The cohort divided into two groups according to PVS score which was made manually. Radiomics evaluation of NAWM was implemented on centrum level. Least absolute shrinkage and selection operator (LASSO) logistic regression analysis was used to simplify relevant features with PVS score. Receiver operating characteristic (ROC) curve were used to evaluate the discriminative ability of constructed Radiomics model.

Results: A Radiomics model was constructed using simplified features by LASSO logistic regression analysis. Radiomics model for both FLAIR and $\mathrm{ADC}$ images were found to be efficient for discrimination between low and high level of PVS scores. AUC was obtained as 0.762 (95\% CI: 0.678-0.833) in FLAIR training group, 0.639 (95\% CI: 0.457-0.796) in FLAIR test group, 0.710 (95\% CI: $0.623-0.788$ ) in ADC training group and 0.604 (95\% CI: $0.422-0.767)$ in ADC test group.

Discussion \& Conclusion: The radiomics features variation of the NAWM between patients with low and high level of PVS score may be the reflection of PVS presence and white matter hyperintensities undetectable at macroscopic level.

\section{1-P10}

GLIOBLASTOMA RADIOMICS TO PREDICT SURVIVAL: DIFFUSION CHARACTERISTICS OF SURROUNDING NONENHANCING TISSUE TO SELECT PATIENTS FOR EXTENSIVE RESECTION

LUCA PASQUINI $^{1}$, Alberto Di Napoli ${ }^{1}$, Antonio Napolitano ${ }^{2}$, Francesco Dellepiane $^{1}$, Andrea Romano ${ }^{1}$, Andrei Holodny ${ }^{3}$, Alessandro Bozzao ${ }^{1}$ 
${ }^{1}$ Neuroradiology Unit, NESMOS Department, Sant'Andrea Hospital, La Sapienza University, Via di Grottarossa 1035, Rome 00189, Italy, 2 Medical Physics Department, Bambino Gesù Children's Hospital, IRCCS, Piazza di Sant'Onofrio, 4, Rome 00165, Italy, ${ }^{3}$ Neuroradiology Service, Department of Radiology, Memorial Sloan Kettering Cancer Center, 1275 York Ave, New York, NY 10065, USA

Keywords: glioblastoma, radiomics, surgery, non-enhancing tumor, ADC

Background \& Purpose: Glioblastoma (GBM) is an aggressive primary CNS neoplasm with poor overall survival (OS) despite standard of care. On MRI, GBM is usually characterized by an enhancing portion (which is the surgery target) and a non-enhancing surrounding, representing an admixture of tumor and vasogenic edema. Extent of resection is a long debated issue in GBM, with recent evidence suggesting that both enhancing and non-enhancing tumor (NET) should be resected in the specific group of patients $<65$ years old, regardless of other risk factors (such as molecular biomarkers). Our aim was to test a radiomic model for patient survival stratification in this age group by analyzing MRI features of NET, to aid tumor resection.

Methods: Sixty-eight adult patients were selected with the following criteria: $<65$ years old, pathologic diagnosis of GBM, extensive contrast-enhancing tumor (CET) resection $(>80 \%)$. Extent of resection was evaluated by manually segmenting the enhancing tumor on volumetric T1-weighted MR images before and after surgery (within $72 \mathrm{~h}$ ). All patients underwent the same treatment protocol including standard chemoradiation. Radiomic features were extracted with a custom version of Pyradiomics from the NET. Feature selection was performed with principal component analysis (PCA). A cox regression model was exploited to test the effect of all PCA components on survival. In a second analysis, t-test followed by logistic regression was used to identify the best principal component to discriminate overall survival at 12 months. Statistical significance was set at $\mathrm{p}<.05$. The most relevant features were identified from the component matrix

Results: Five PCA components (PC1-5) explained $90 \%$ of the variance. Only PC5 resulted significant in the cox model, with $\mathrm{p}=.002$ and $\exp (\mathrm{B})=0.686$. PC5 resulted significant in discriminating survival at $\mathrm{t}$-test $(\mathrm{p}=.002)$ and logistic regression analysis $(\mathrm{p}=0.006)$. ADC-based features were the most significant for patient survival stratification, demonstrating high relevance for PC5.

Conclusions: Our study demonstrates that ADC radiomic features from the NET can boost survival stratification of GBM patients. ADC-based textural, shape and fractal features demonstrated high performance in our analysis, possibly reflecting cell proliferation in the area surrounding the tumor mass. These results could be used to improve patient selection for more extensive surgery, identifying candidates based on expected survival.
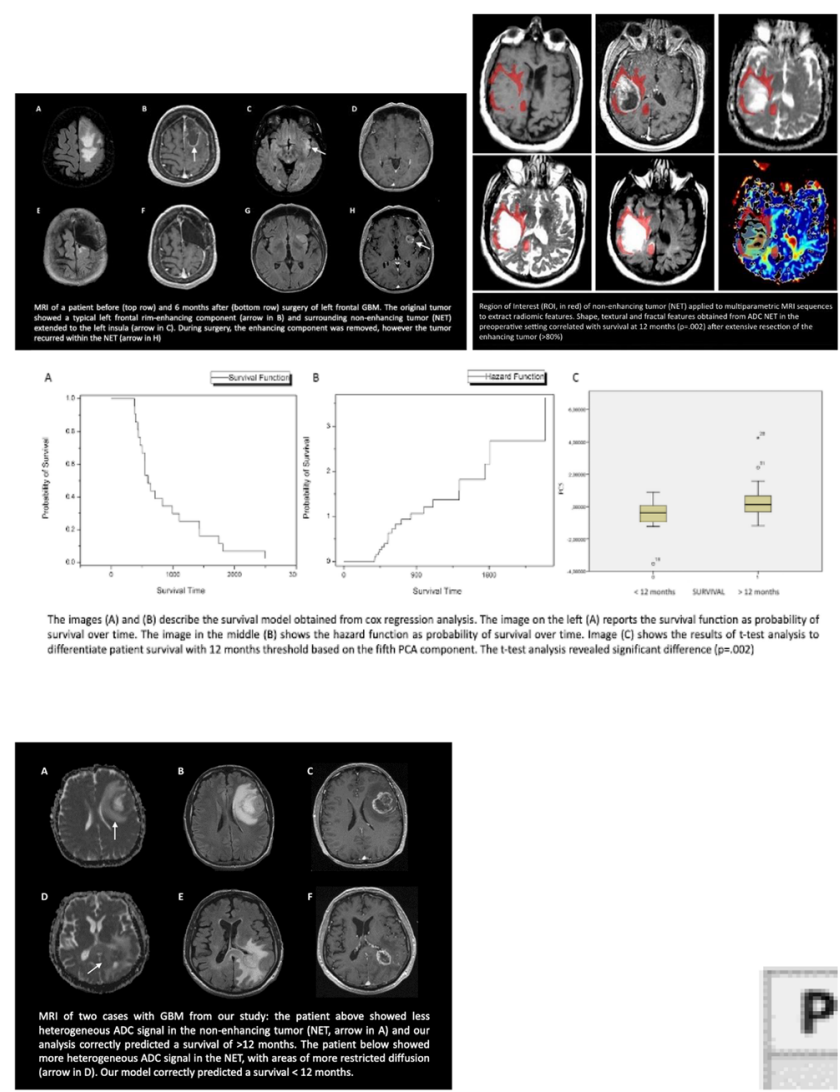

1-P11

CORRELATION BETWEEN LIFELONG DYNAMICS OF PSYCHOPHYSIOLOGICAL PERFORMANCE AND BRAIN MORPHOLOGY

YAUHEN STATSENKO $^{1}$, Tetiana Habuza ${ }^{2}$, Liaisan Uzianbaeva ${ }^{3}$, Klaus Neidl Van Gorkom ${ }^{1}$, Maroua Belghali ${ }^{4}$, Inna Charykova ${ }^{5}$, Milos Ljubisavljevic $^{2}$, Darya Smetanina ${ }^{2}$, Gillian Lylian Simiyu ${ }^{2}$, Tetiana Kurbatova $^{2}$, Daria Luksho ${ }^{2}$, Miklos Szolics ${ }^{6}$, Fatmah Al Zahmi ${ }^{7}$, Taleb M. Almansoori ${ }^{2}$, Jamal Al Koteesh ${ }^{8}$

${ }^{1}$ College of Medicine and Health Sciences, Al Ain, UAE,${ }^{2}$ College of IT, UAE University, Al Ain, UAE, ${ }^{3}$ Wayne State University, Department of Biomedical Engineering, Detroit, MI, ${ }^{4}$ College of Education, Department of Health and Physical Education, UAE University, Al Ain, UAE, ${ }^{5}$ Laboratory of Psychology, Republican Scientific-Practical Center of Sports, Minsk, Belarus, ${ }^{6}$ Division of Neurology, Department of Medicine, Tawam Hospital, ${ }^{7}$ Mediclinic Middle East Parkview hospital, Dubai, UAE, ${ }^{8}$ Radiology Department, Tawam Hospital, Al Ain, UAE

Keywords: brain structural-functional association, psychophysiological test, brain morphology

Background: An association between brain structural changes and cognitive decline is an issue of ongoing studies.

Objectives: To assess a correlation between volumetric changes of brain structures and psychophysiological performance in age groups; to assess reliability of psychophysiological tests.

Materials \& Methods: In a cross-sectional study of 215 healthy participants we performed (i) volumetric segmentation of brain regions on T1- 
weighted MRI images and expressed their volume as a percentage of the intracranial volume; (2) psychophysiological tests to assess performance of cognitive domains. We represented age-related patterns for the following variables: simple visual-motor reaction (SVMR), complex visualmotor reaction (CVMR), attention study technique (AST), interference resilience technique (IRT), reaction to a moving object (RMO), decision making time (DMT). Then we estimated a correlation between volumetric changes of brain compartments and dynamics of psychophysiological performance.

Results: There is a strong positive association between the total volume of cerebrospinal fluid (CSF\%) and the latency of reacting to presented stimuli in SVMR, CVMR, IRT, AST tests. A positive dependency is also observed for the volume of brain ventricles and reaction time in the tests. Both CSF\% and the intraventricular volume (iCSF\%) are positively correlated with age (r-values are 0.8 and 0.56 respectively). The strongest association between structural changes and functional outcomes is between CSF\% and reaction time in IRT test which is most cognitively demanding in our study $(r=0.36)$. The test utilizes a set of cognitive domains such as attention, memory, and executive functioning with its subdomains of task switching, inhibitory control, and updating. DMT test, that reflects task switching and inhibitory control subdomains, is associated positively with the CSF\% $(r=0.16)$, iCSF\% $(r=0.14)$, and negatively with gray matter (GM\%) and its cortex $(\mathrm{cGM} \%)(\mathrm{r}=-0.13$ for both GM\% and cGM\%).

Mean reaction time in the most primitive test of our battery (SVMR test) has a positive association with CSF\% $(r=0.14)$ and GM\% $(r=0.16)$ in contract to the relationship with WM\% $(r=-0.14)$. Better brain connectivity in individuals with a high proportional volume of the total white matter may account for the faster reaction time.

Conclusion: Our observations justify the reliability of the psychophysiological tests used because decision-making processes are undoubtedly done in the GM cortex. Despite this, the weak associations of DMT with CSF\% ( $r=0.16)$ limits the utility of DMT as a marker of brain atrophy.

\section{1-P12}

MODELS OF BRAIN COGNITIVE AND MORPHOLOGICAL CHANGES ACROSS THE LIFE: MACHINE LEARNING-BASED APPROACH

TETIANA HABUZA ${ }^{1}$, Yauhen Statsenko ${ }^{2}$, Liaisan Uzianbaeva ${ }^{3}$, Klaus Neidl Van Gorkom ${ }^{2}$, Nazar Zaki ${ }^{1}$, Maroua Belghali ${ }^{4}$, Inna Charykova ${ }^{5}$, Miklos Szolics ${ }^{6}$, Milos Ljubisavljevic ${ }^{2}$, Darya Smetanina ${ }^{2}$, Gillian Lylian Simiyu $^{2}$, Tetiana Kurbatova ${ }^{2}$, Daria Luksho ${ }^{2}$, Fatmah Al Zahmi ${ }^{7}$, Taleb M. Almansoori ${ }^{2}$, Jamal Al Koteesh ${ }^{8}$

${ }^{1}$ College of IT, UAE University, Al Ain, UAE, ${ }^{2}$ College of Medicine and Health Sciences, Al Ain, UAE, ${ }^{3}$ Wayne State University, Department of Biomedical Engineering, Detroit, MI, ${ }^{4}$ College of Education, Department of Health and Physical Education, UAE University, Al Ain, UAE, ${ }^{5}$ Laboratory of Psychology, Republican Scientific-Practical Center of Sports, Minsk, Belarus, ${ }^{6}$ Division of Neurology, Department of Medicine, Tawam Hospital, Al Ain, UAE, ${ }^{7}$ Mediclinic Middle East Parkview hospital, Dubai, UAE, ${ }^{8}$ Radiology Department, Tawam Hospital, Al Ain, UAE

Keywords: cognition, brain morphometry, aginig

Background: Reciprocal structural and functional changes of the brain during active neurodevelopment, maturation and decline are poorly understood.
Objective: To analyze and design descriptive models of brain cognitive and morphological changes across the lifespan.

Material \& Methods: We did a cross-sectional study of 215 healthy participants (4-84 yo) of four age groups: adolescents $(<20$ years, $\mathrm{n}=43$ ), young adults $(20-39, \mathrm{n}=58)$, midlife adults $(40-59, \mathrm{n}=59)$, and older adults $(\geq 60, n=55)$. The participants passed psychophysiological tests (PTs) and underwent brain MRI. We segmented images acquired in T1W and FLAIR sequences to calculate the percentage of the following compartments to the total intracranial volume (TIV): gray matter (GM\%), white matter (WM\%), cerebrospinal fluid (CSF\%).

White matter hyperintensities (WMH\%) were also assessed in proportion to TIV. To describe brain structural changes throughout life we used polynomial repression machine learning models of degree one and two. We resorted to T-test to compare slopes for brain structural and functional changes within the age groups. Kruskal-Wallis test (KWT)was employed to contrast the distribution of group data with the entire cohort.

Results: We observe a significant increase in CSF\% in young and older adults ( $\mathrm{p}=0.00405$ and $1.04 \mathrm{e}-08$ ). There is a steady decrease of TIV from adolescence through young and midlife age to old age (KWT: $\mathrm{p}=0.0068$ ). A rise in WM\% is significant in adolescents $(\mathrm{p}=0.00115)$ and it is steeper than expansion of CSF\% $(\mathrm{p}=0.033)$. In older adults, the dynamics of WM\% is significantly shallower than the one of CSF\% ( $=2.2 \mathrm{e}-16)$. The rate of accumulation of WM\% is the highest during adolescence, and then it reduces slowly throughout the years. Results of the PTs that reflect information processing speed follow a 'U-shaped' pattern, while estimates of task switching and inhibitory control follow a linear pattern. In adolescents, slopes of all the PTs variables are negative and abruptly steep ( $p$ ranges 2.6e-07-0.0475), while in young adults the slopes are positive and gradually steep. There is a slight increase in WMH\% across the lifespan (p ranges $0.379-0.868$ ).

Conclusion: WM\% changes in an inverted U-shaped pattern, while GM\% follows a regular parabola shape. This may give a wrong impression of the invertibility of the GM\% loss. To avoid the misconception, we recommend modeling and representing GM\% with polynomial functions of degree three or higher, for instance, cubic functions. WMH\% should not be considered as a marker of physiologic brain aging but rather as a sign of cerebrovascular pathology.

\section{1-P13}

EFFECT OF SEX ON AGE-RELATED CHANGES IN BRAIN MORPHOLOGY

LIAISAN UZIANBAEVA ${ }^{1}$, Yauhen Statsenko ${ }^{2}$, Tetiana Habuza ${ }^{3}$, Klaus Neidl Van Gorkom ${ }^{2}$, Maroua Belghali ${ }^{4}$, Inna Charykova ${ }^{5}$, Darya Smetanina $^{2}$, Gillian Lylian Simiyu ${ }^{2}$, Tetiana Kurbatova ${ }^{2}$, Daria Luksho $^{2}$, Miklos Szolics ${ }^{6}$, Milos Ljubisavljevic ${ }^{2}$, Fatmah Al Zahmi ${ }^{7}$, Taleb M. Almansoori ${ }^{2}$, Jamal Al Koteesh ${ }^{8}$

${ }^{1}$ Wayne State University, Department of Biomedical Engineering, Detroit, MI, USA, ${ }^{2}$ College of Medicine and Health Sciences, Al Ain, UAE, ${ }^{3}$ College of IT, UAE University, Al Ain, UAE, ${ }^{4}$ College of Education, Department of Health and Physical Education, UAE University, Al Ain, UAE, ${ }^{5}$ Laboratory of Psychology, Republican Scientific-Practical Center of Sports, Minsk, Belarus, ${ }^{6}$ Division of Neurology, Department of Medicine, Tawam Hospital, ${ }^{7}$ Mediclinic Middle East Parkview hospital, Dubai, UAE, ${ }^{8}$ Radiology Department, Tawam Hospital, Al Ain, UAE

Keywords: voxel-based morphometry, brain aging, sex differences 
Background: To date, little is known about the effect of sex on agerelated changes in brain morphology. Recent studies on this issue ended up with discrepant findings insufficient to work out a common theory.

Objectives: To study sex differences in lifelong changes of the brain volumetric data.

Materials \& Methods: A cross-sectional study of 215 healthy participants of whom 131 females and 84 males were divided into groups: adolescents $(<20$ years, $n=18 / 25)$, young adults $(20-39, n=35 / 23)$, midlife adults $(40$ $59, \mathrm{n}=38 / 21)$, and older adults $(\geq 60, \mathrm{n}=40 / 15)$. All participants underwent brain MRI (T1W, FLAIR). Acquired images were studied with voxelbased morphometry and segmented into parts. Then we calculated volumes of each of the following compartment as a fraction of the total intracranial volume (TIV): the total (GM\%) and cortical gray matter (cGM\%), the total (CSF) and intraventricular cerebrospinal fluid (iCSF), the total white matter (WM\%) and its lesions hyperintensive on FLAIR (WMH\%). The exclusion criteria were as listed: organic brain pathology, mental disorders, past medical history of head injuries, and any radiologic signs of neurodegenerative diseases on MRI. We assessed differences between brain morphometry data for females and males with Mann-Whitney U-test.

Results: GM\% is significantly higher in women compared with men in middle-aged individuals ( $31.85 \pm 3.96 \%$ vs $29.46 \pm 2.86 \% ; \mathrm{p}=0.009)$ and older adults $(30.94 \pm 3.65 \%$ vs $28.87 \pm 2.38 \%$; $\mathrm{p}=0.0145)$. cGM $\%$ differs significantly between sexes in the midlife adults $(21.64 \pm 3.68 \%$ vs 19.54 $\pm 2.91 \% ; \mathrm{p}=0.0142$ ). Atrophy of the gray matter accounts for a difference in GM-to-WM ratio between sexes. The difference in the ratio is significant in the midlife adults $(77.43 \pm 15.81$ vs $70.17 \pm 14.65 ; \mathrm{p}=0.0375)$ and oversignificant in the older adults $(76.18 \pm 17.77$ vs $68.22 \pm 12.14$; $\mathrm{p}=0.0741)$. A marked sex difference in CSF\% $(22.68 \pm 3.33 \%$ vs 26.79 $\pm 4.94 \% ; \mathrm{p}=0.0036)$ and $\mathrm{iCSF} \%(1.75 \pm 0.79 \%$ vs $2.54 \pm 1.02 \% ; \mathrm{p}=0.0033)$ reveals that elderly men are prone to age-related brain atrophy much more than women of the same age. A difference between sexes in the iCSF-toCSF ratio is also significant after the age of 60 years $(7.59 \pm 2.72 \%$ vs 9.25 $\pm 2.7 \%$; $\mathrm{p}=0.0076$ )

Conclusion: After being adjusted to the skull volume, relative volumes of brain compartments (e.g., GM\%, WM\%, CSF\%, etc.) do not differ significantly for both sexes till 40 years of age. Our observations can be explained by sex difference either in the speed or onset of atrophic changes in the gray matter. They either start earlier or go faster in males versus females. Elderly men are prone to age-related brain atrophy much more than women of the same age.

\section{1-P14}

DEEP LEARNING CAN DIFFERENTIATE IDH-MUTANT FROM IDH-WILD TYPE GBM

LUCA PASQUINI $^{1,2}$, Alberto Di Napoli ${ }^{2}$, Antonio Napolitano ${ }^{3}$, Martina Lucignani $^{3}$, Emanuela Tagliente ${ }^{3}$, Francesco Dellepiane ${ }^{2}$, Andrea Romano $^{2}$, Alessandro Bozzao ${ }^{2}$

${ }^{1}$ Neuroradiology Unit, Radiology Department, Memorial Sloan Kettering Cancer Center, 1275 York Ave, New York, NY 10065, USA, ${ }^{2}$ Neuroradiology Unit, NESMOS Department, Sant'Andrea Hospital, La Sapienza University, Via di Grottarossa 1035, Rome 00189, Italy, ${ }^{3}$ Medical Physics Department, Bambino Gesù Children's Hospital, IRCCS, Piazza di Sant'Onofrio, 4, Rome 00165, Italy

Keywords: Glioblastoma, MRI, Artificial Intelligence, Deep Learning

Background: Mutations of isocitrate dehydrogenase (IDH) are frequent in secondary glioblastoma (GBM) $(73 \%)$ and rare in primary GBM
(3.7\%), leading to better prognosis and longer survival. Distinction of IDH mutant and wildtype GBMs may be challenging on MRI, since conventional imaging features show considerable overlap. Previous studies attempted IDH prediction by means of supervised machine learning, with limited suitability in the clinical practice. While few studies employed unsupervised learning in a mixed low/high grade glioma population, a GBM-specific model is still lacking in the literature. Our objective was to develop an automatic model for IDH prediction in GBM by using Convoluted Neural Networks (CNN) on MRI data.

Methods: We included 156 adult patients with pathologically proven GBM. MRI data included: unenhanced T1, T2, FLAIR, enhanced MPRAGE images, rCBV maps from DSC perfusion, ADC maps from DWI. Tumor area was obtained by a bounding box function on the axial slice with widest tumor extension on T2 images and was projected on every sequence. Data was split into training and validation $(80: 20)$ sets. Augmentation techniques allowed to fix class unbalance. A 4 block 2D - CNN architecture was implemented for IDH prediction on every MRI sequence. The pipeline was written in Python3 and was implemented on Jupyter Notebook, using the Keras API. IDH mutation probability of each sample was calculated with softmax activation function from the last dense layer. Categorical cross entropy loss (CCEL) with two output nodes was evaluated on each epoch. Highest performance was calculated accounting for model accuracy and CCEL in the validation cohort.

Results: To evaluate individual predictive performance, networks were trained for 1500 epochs separately on each MRI sequence. Our model achieved the following performance: T1 (accuracy 82\%, AUC 0.8, CCEL 1.12), T2 (accuracy $82.3 \%$, AUC 0.64, CCEL 1.56), FLAIR (accuracy $85 \%$, AUC 0.86, CCEL 3.12), MPRAGE (accuracy 80\%, AUC 0.62, CCEL 2.26), rCBV (accuracy 86\%, AUC 0.88, CCEL 0.72). ADC achieved lower performance.

Conclusion: We built a GBM-tailored deep-learning model for IDH mutation prediction, achieving accuracy of $85 \%$ with FLAIR images and $86 \%$ with rCBV maps. High predictivity of perfusion images may reflect the known correlation between IDH, hypoxia inducible factor (HIF) and neoangiogenesis. The lower performance of $\mathrm{ADC}$ may be due to uniform values across GBM or confounding factors unrelated to IDH such as presence of blood products. The presented model may set a path for non-invasive evaluation of IDH mutation in GBM.

\section{1-P15}

COMPARISON OF BRAIN VOLUMETRIC CHANGES WITH FUNCTIONAL OUTCOMES IN PHYSIOLOGIC BRAIN AGING

KLAUS NEIDL VAN GORKOM ${ }^{1}$, Yauhen Statsenko ${ }^{1}$, Tetiana Habuza $^{2}$, Liaisan Uzianbaeva ${ }^{3}$, Maroua Belghali ${ }^{4}$, Inna Charykova ${ }^{5}$, Milos Ljubisavljevic ${ }^{2}$, Darya Smetanina ${ }^{2}$, Gillian Lylian Simiyu ${ }^{2}$, Tetiana Kurbatova ${ }^{2}$, Daria Luksho ${ }^{2}$, Miklos Szolics ${ }^{6}$, Fatmah Al Zahmi $^{7}$, Taleb M. Almansoori ${ }^{2}$, Jamal Al Koteesh ${ }^{8}$

${ }^{1}$ College of Medicine and Health Sciences, Al Ain, UAE, ${ }^{2}$ College of IT, UAE University, Al Ain, UAE ${ }^{3}$ Wayne State University, Department of Biomedical Engineering, Detroit, MI, ${ }^{4}$ College of Education, Department of Health and Physical Education, UAE University, Al Ain, UAE, ${ }^{5}$ Laboratory of Psychology, Republican Scientific-Practical Center of Sports, Minsk, Belarus, ${ }^{6}$ Division of Neurology, Department of Medicine, Tawam Hospital, ${ }^{7}$ Mediclinic Middle East Parkview hospital, Dubai, UAE, ${ }^{8}$ Radiology Department, Tawam Hospital, Al Ain, UAE

Keywords: brain morphometry, cognitive performance, slopes 
Background: Both brain structure and cognitive function undergo significant changes throughout life. Particular interest remains in establishing an association between structural and functional changes in normal brain aging.

Objective: To identify structural-functional association of brain changes across the lifespan; to compare the slopes of psychophysiological performance with slopes of structural brain changes.

Materials \& Methods: 215 healthy participants, divided into four age groups, underwent brain MRI with voxel-based morphometry and lesion segmentation. By utilizing nonparametric statistics and machine learning we analyzed structural changes of the major brain compartments and modeled dynamics of neurofunctional performance throughout the years. We considered eight structural estimates: total intracranial volume, total and intraventricular cerebrospinal fluid, total and cortical gray matter, white matter and lesions hyperintensive in FLAIR MRI sequence. Cognitive performance was assessed with a psychophysiological battery that provides values for seven dependent variables: five of them represent mean reaction time in taskswith distinct paradigms, and two more tests reflect a latency caused by task switching and inhibitory control. We tested linear models of structural and functional changes for significant differences between slopes in age groups with T-test. Slopes for each of seven dependent variables of psychophysiological tests were compared with eight slopes for structural estimates.

Results: There is no significant difference between the slopes for the functional and brain volumetric changes in midlife adults aged 40-60 years ( $\mathrm{p}$-value ranges from 0.116 to 0.965 ). In other age groups the dynamic of the test results significantly differs from that for brain volumetric data ( $p$-value ranges from $9.10 \times 10-11$ to 0.046 ) . The portion of significant differences between the slopes for structural and functional estimates is the highest in adolescents aged 4-20 years (46 out of 56; 82\%); and moderate in young adults aged $20-40$ years $(28 ; 50 \%)$ and in older adults aged 60 years and above $(24 ; 43 \%)$.

Conclusion: Prominent neurodevelopmental changes account for the disproportional changes in the brain structure and cognitive functioning in adolescents. A continuous accumulation of skills and educational experience justifies the same tendency in young adults. Due to the ongoing process of acquiring new knowledge till the age of over 40 years, the cognitive performance of individuals may still improve at the time when irreversible structural changes have already taken place. Dramatic changes both in the brain compartments and in the cognitive performance lead to significant differences in dynamics of the structural atrophy and functional decline.

\section{1-P16}

CASE REPORT: ACUTE DISSEMINATED ENCEPHALOMYELITIS (ADEM) ASSOCIATED WITH COVID -19 INFECTION

\section{$\underline{\text { XIAO CHING LIEW }}^{1}$, Khariah Binti Mat Nor ${ }^{2}$, Emy Saera Binti Rosani ${ }^{1}$}

${ }^{1}$ Department of Radiology, Hospital Sungai Buloh, Selangor, Malaysia, ${ }^{2}$ Imaging Unit, Medical Faculty, University Technology MARA, Sungai Buloh, Selangor, Malaysia

Keywords: Acute Disseminated Encephalomyelitis, COVID-19, Brain

Introduction: Coronavirus disease- 2019 (COVID-19) predominantly affects respiratory system. However, multiple neurological manifestations have also been reported. We report a case of acute disseminated encephalomyelitis in COVID-19 infection.
Case presentation: Thirty-two-year-old gentleman who was previously healthy, presented with sudden onset of slurred speech and choking episodes. His RT-PCR swab test for COVID-19 infection was positive 3 days prior with history of fever for a day, without respiratory symptom. His chest radiograph was normal. On examination during admission, he was fully conscious with dysarthria, left facial weakness sparing forehead and absent gag reflex. The initial plain CT Brain showed multiple illdefined white matter hypodensities at both fronto-parietal lobes. CT venogram showed no evidence of venous sinus thrombosis. However, he became progressively aphasic, confused and developed new weakness of left upper and both lower limbs two days later. Follow-up CT Brain revealed new hypodense lesions involving corpus callosum. Subsequently MRI Brain depicted multiple bilateral, almost symmetrical, subcortical white matter T1W hypointensity with T2W/FLAIR hyperintensity, in both centrum semiovale, corona radiata, periventricular regions and corpus callosum. These lesions demonstrated diffusion restriction on ADC/DWI mapping, suggestive of cytotoxic oedema. Cortical swelling associated with abnormal T2W/FLAIR hyperintensity are seen at both medial temporal lobes. None of these lesions show enhancement. C-Reactive-Protein was elevated $(2.8 \mathrm{mg} / \mathrm{dL})$. Cerebrospinal fluid(CSF) analysis demonstrated clear appearance and normal glucose with slightly elevated protein $(0.49 \mathrm{~g} / \mathrm{L})$. Bacterial and fungal culture of CSF were negative. Oligoclonal bands were not detected in both serum and CSF examination. Electroencephalogram (EEG) showed normal brain activity.

He was treated with Aspirin 150mg once daily and subcutaneous Enoxaparin $60 \mathrm{mg}$ once daily, in addition to intravenous Acyclovir $500 \mathrm{mg}$ in three divided doses and intravenous Methylprednisolone $1 \mathrm{~g}$ once daily for 5 days. Physiotherapy and speech therapy commenced. He was discharged after 20 days of hospitalization with improvement in consciousness but unchanged neurological deficit.

Discussion: This patient presented with stroke-like symptoms which do not conform to specific vascular territories. The basal ganglia, brain stem and cerebellum are spared. No evidence of acute haemorrhage, cerebral venous sinus thrombosis or deep gray matter involvement. These features are suggestive of acute disseminated encephalomyelitis. It could be postinfectious or immune- mediated response to COVID-19. Bilateral medial temporal lobes involvement favors limbic encephalitis.

Conclusion: In view of rising number of cases with neurological manifestations in COVID- 19 infection, more research evidence on neuroimaging findings should be established, in order to improve diagnostic confidence among radiologists.
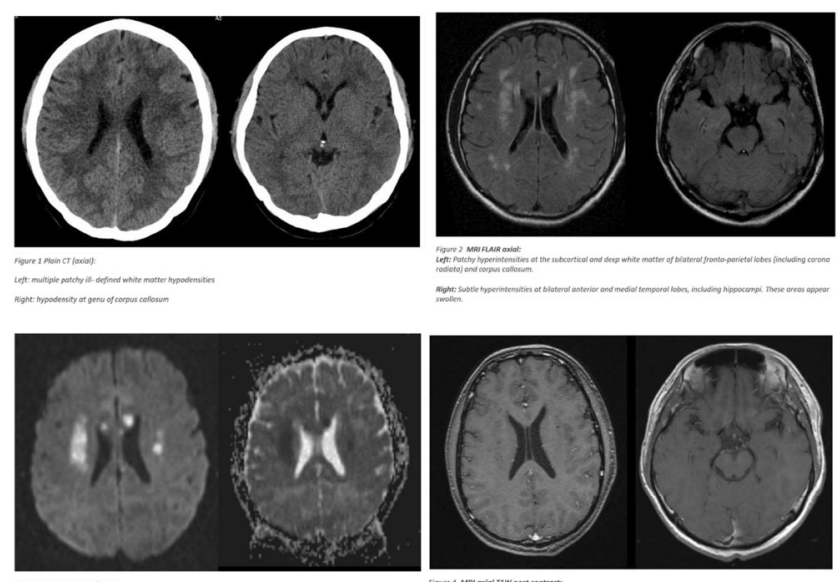

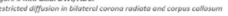

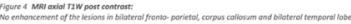




\section{1-P17}

ICOBRAIN MS 5.1: A COMBINED APPROACH FOR IMPROVING THE DETECTION OF MULTIPLE SCLEROSIS LESIONS

MLADEN RAKIC $^{1}$, Sophie Vercruyssen ${ }^{1}$, Simon Van Eyndhoven ${ }^{1}$, $\overline{\text { Ezequiel de la Rosa }}^{1}$, Dirk Smeets ${ }^{1}$, Diana Sima ${ }^{1}$

${ }^{1}$ icometrix, Leuven, Belgium

Keywords: Multiple sclerosis, Lesion segmentation, Deep-learning, Attention-gate U-net, Combined classification, Unsupervised machine learning

Purpose: Development of a robust method for improving the segmentation and classification of multiple sclerosis (MS) brain lesions.

Methods / Background: The dataset consists of 159 real-world multicenter pre-contrast T1 and FLAIR scans from MS patients, together with manual delineations of four spatially differentiated classes of white matter lesions: infratentorial (IT), periventricular (PV), juxtacortical (JC) and deep white matter (DWM), segmented and validated by a panel of raters. Careful data stratification, which preserves data variability over training, validation and testing sets and minimizes potential bias during training, was performed to select 93 training, 15 validation and 51 testing subjects. Medical device software icobrain ms 5.0 was used as a baseline to assess the performance on the test set. We developed icobrain ms 5.1, which incorporates deep learning in the form of an attention-gate 3D U-net to address the challenging detection of IT and JC lesions. icobrain ms 5.0 and attention-gate 3D U-net were fused in an ensemble fashion. Detection and classification performance is evaluated with lesion-wise Dice score.

Results / Findings: icobrain ms 5.0 achieved lesion-wise Dice scores of $0.34,0.77,0.31$ and 0.71 for IT, PV, JC and DWM lesions, respectively. Following the same order, the attention-gate U-net yielded scores of 0.48 , $0.69,0.59$ and 0.70 . The ensemble method, which fuses the two methods' outputs by selecting segmented lesions from a pipeline that achieved significantly better performance evaluated on the associated class, achieved $0.48,0.77,0.62$ and 0.69 when evaluated on the test set.

Conclusion: Results obtained with a novel attention-gate U-net illustrate the improvement in detection of IT lesions by $14 \%$ and JC lesions by $28 \%$. The ensemble method offered a 'best-of-both-worlds' performance, with satisfying results for MS lesion segmentation.

\section{1-P18}

INTRADURAL PREPONTINE CHORDOMA MIMICKING AN EPIDERMOID CYST ON MAGNETIC RESONANCE IMAGING

\section{GONCALO ALMEIDA $^{1}$, Goncalo Januario ${ }^{2}$, Rui Carvalho ${ }^{1}$}

${ }^{1}$ Department of Neuroradiology, Centro Hospitalar e Universitario de Lisboa Central, Lisbon, Portugal, ${ }^{2}$ Department of Neurosurgery, Centro Hospitalar e Universitario de Lisboa Central, Lisbon, Portugal

Keywords: Chordoma, Epidermoid Cyst, Magnetic Resonance Imaging

Introduction: Chordomas are relatively rare malignant tumors derived from embryonic notochord remnants. Most intracranial chordomas show extradural extension and cause bone erosion. However, a small percentage of these tumors are exclusively intradural and tend to show less aggressive features, although local recurrence and metastatic spread have been described. Intradural chordomas with imaging features similar to epidermoid cysts (EC) are exceedingly rare.
Methods: We describe the case of a nondestructive intradural prepontine chordoma mimicking an EC on magnetic resonance imaging (MRI) in a 44-year-old male patient.

Results: A 44-year-old man with unremarkable medical history presented with acute-onset vertigo and vomiting. The neurological examination and full laboratorial work-up showed no abnormalities.

Due to suspicion of a posterior fossa lesion and lower sensitivity of computed tomography (CT) scans for its depiction, the patient underwent MRI as first-choice imaging technique. The MRI revealed an extra-axial space-occupying cystic lesion centred in the prepontine cistern, with no evidence of lytic bone destruction. The lesion was hypointense on T1weighted imaging (T1WI), hyperintense on T2-weighted imaging (T2WI) and showed restricted diffusion with high signal intensity on non-echoplanar diffusion-weighted imaging (DWI) and low signal intensity on the apparent diffusion coefficient (ADC) map. In addition, no enhancement was observed after intravenous gadolinium injection. The lesion caused mass effect on the brainstem, encased the basilar artery and contacted the left facial and both trigeminal nerves. Based on these findings, a presumptive diagnosis of EC was made.

The patient underwent surgery through a left retromastoid approach and subtotal resection of the lesion was performed, due to its close proximity with the basilar artery and cranial nerves. Pathological examination of the surgical specimen disclosed a well-differentiated chordoma with reduced mitotic activity $(\mathrm{Ki}-67$ index $<10 \%)$. Due to the presence of a tumor remnant on the postoperative imaging assessment, adjuvant radiation therapy was proposed.

Discussion \& Conclusion: This case report highlights the need to include intradural chordomas in the differential diagnosis of a nonenhancing and nondestructive intradural prepontine lesion demonstrating restricted diffusion on MRI. Gross total resection, adjuvant radiation therapy in cases of macro/microscopical residual disease and regular follow-up are warranted due to the possibility of local recurrence and metastatic dissemination.

\section{1-P19 \\ IMAGING ASSESSMENT OF IMMUNOTHERAPY TREATMENT- RELATED NEUROTOXICITY}

THYAGO OLIVEIRA DE QUEIROZ ${ }^{1}$, Matheus Dorigatti Soldatelli ${ }^{1}$, Lazaro Luis Faria do Amaral

${ }^{1}$ BP - A Beneficencia Portuguesa de Sao Paulo, Brazil

Keywords: Immunotherapy, neurotoxicity, neurological immune-related adverse effects

Introduction: Immunotherapy enhances a patient's own immune system to fight against tumor cells, and their neurotoxicity, referred as neurological immune-related adverse effects (irAE), is an inflammatory reaction that attacks healthy tissue. These include alterations in the central and peripheral nervous system, and the awareness of the neuroradiological findings is crucial for diagnostic work-up and adequate therapeutic measures.

Methods: Case-based review of MRI findings in a serie of patients who presented neurological irAE in our institution.

Results: Peripheral nervous system irAE represented by left orbital myositis (figure 1 coronal $\mathrm{T} 1$ post-contrast: inferior and lateral orbital rectus muscles are edematous and show increased enhancement compared to the contralateral side). 
Central nervous system irAE characterized by: hypophysitis (figure 2 sagittal T1 post-contrast: diffusely thickened pituitary, larger than expected for age, demonstrating avid enhancement), rhombencephalitis (figure 3 axial FLAIR post-contrast \& figure 4 Arterial Spin Labelling - ASL: abnormal leptomeningeal enhancement and significant hyperperfusion in ASL, at both cerebellar hemispheres) and aseptic meningitis (figure 5 axial FLAIR post-contrast: nodular leptomeningeal enhancement). All patients had nearly complete resolution of the previous MRI finding after suspension of the immunotherapy.

Discussion: Neurological irAE have been noted more and more in patients suffering from cancer and treated with immunotherapy, as it has become increasingly popular during the last decade. Compared to the frequency of irAE in other organs, neurological complications are rather low and affect more the peripheral than the central nervous system. Some of them include radiculopathies, neuropathies, myopathies, hypophysitis, aseptic meningitis, and encephalitis.

Neuroradiologist has an essential role of recognizing the neurological irAE imaging findings and to exclude other underlying reasons, such as progression of disease and infections, as there is a consensus that immunotherapy should be discontinued when the neurological symptoms are attributed to this treatment, to avoid clinical deterioration and irreversible neurological deficits.

Conclusion: Immunotherapy is a powerful therapeutic tool, and many new agents are expected to receive approval and cover a wider range of malignancies. As a result, it is crucial that neuroradiologists keep abreast of the advancements in the treatment of cancer and their neurotoxic effects, once its imaging findings have an important impact on the patient's treatment.
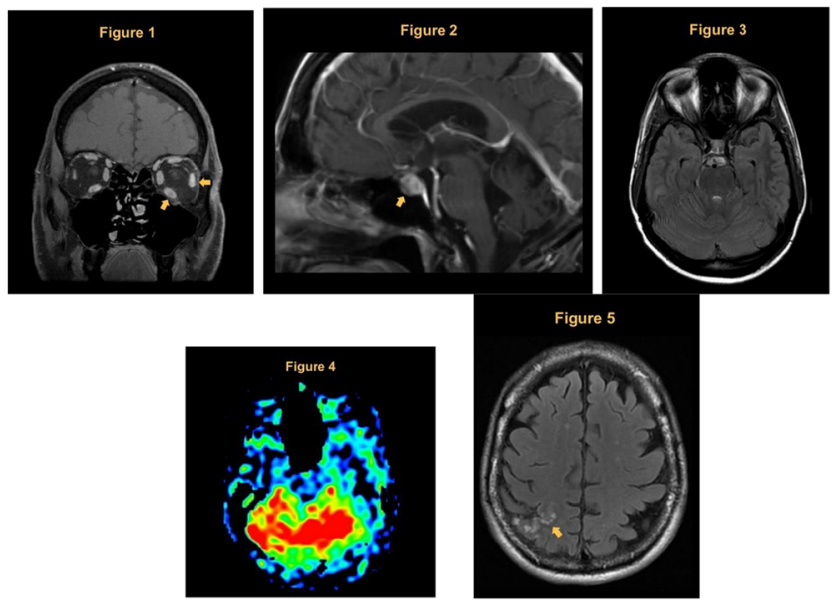

1-P20

INTRA-ANEURYSMAL FLOW PATTERNS IN INTERNAL CAROTID ARTERY ANEURYSMS: AN ILLUSTRATIVE COMPARISON BETWEEN 4D FLOW MR IMAGING AND 3D TOF MR ANGIOGRAPHY

CORRADO SANTAROSA ${ }^{1}$, Olivier Brina ${ }^{1}$, Philippe Reymond ${ }^{1}$, KarlOlof Loevblad $^{1}$, Maria Isabel Vargas Gomez ${ }^{1}$

${ }^{1}$ Division of Neuroradiology, Geneva University Hospitals, Geneva, Switzerland

\section{Keywords: Aneurysm, Hemodynamics, 4D Flow MRI}

Introduction: Hemodynamics of cerebral aneurysms may be related to aneurysm instability. 4D flow MR imaging, based on a 4D phase-contrast
MR (4D PCMR) imaging technique, is increasingly emerging as a reliable method for the actual in vivo assessment of the aneurysmal hemodynamics. This educational poster will describe typical aneurysmal flow patterns of internal carotid artery (ICA) aneurysms as determined on 4D flow MRI. It will also illustrate the correspondences between those 4D PCMR-derived flow patterns and the signal intensity spatial distribution of flow-dependent 3D TOF MR angiography.

Materials \& Methods: A consecutive series of 30 patients with ICA aneurysm have been considered. These patients underwent a 3D rotational angiography (3DRA) and an MRI examination, including 4D PCMR and 3D TOF MRA. 4D PCMR velocity field data and 3D TOF signal intensity data were combined with a vascular geometric scaffold derived from the 3DRA. Paraview software (http://paraview.org/) was used to depict flow patterns through streamlines and analyze the spatial distribution of 4D PCMR velocities and 3D TOF signal intensity.

Results: Illustrative cases of aneurysmal flow patterns will be depicted according to the inflow jet type (concentrated versus diffuse) and the vortex core complexity (simple versus complex), and stability (stable versus unstable). Spatial distribution of the TOF signal intensity will be shown to parallel that of the 4D PCMR-derived velocities, with a neat distinction between the inflow jet and the recirculation zone.

Conclusion: The reader will know how to describe the flow pattern of a cerebral aneurysm and which flow patterns are the most recurring in ICA aneurysms. Additionally, he will be aware that a routinary 3D TOF acquisition, besides the appreciation of the morphology of the aneurysm, may allow inferring some of its hemodynamic properties.

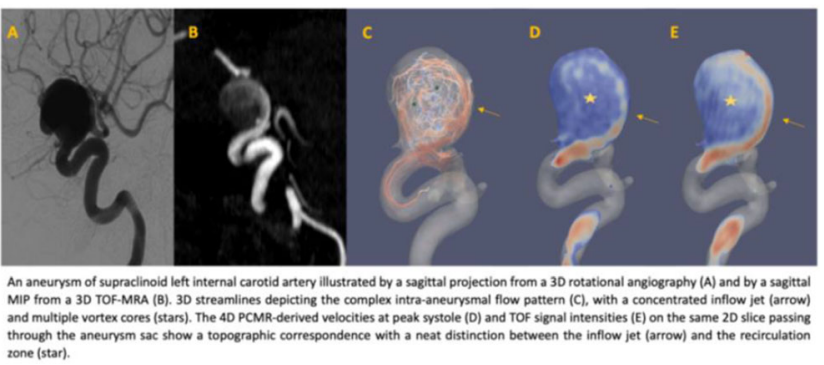

\section{1-P21 \\ BRACHIOCEPHALIC ARTERIES STATE AND WHITE MATTER LESIONS}

ALEXANDER BERDALIN $^{1}$, Vladsilav Bondarenko ${ }^{1}$, Golovin Denis ${ }^{1}$, Anastasia Vishnyakova $^{1}$, Gubskiy Ilya ${ }^{1}$, Vladimir G. Lelyuk ${ }^{1}$

${ }^{1}$ Scientific and research center of radiology and clinical physiology of FSBI «Federal center of brain research and neurotechnologies» of the Federal Medical Biological Agency, Moscow, Russian Federation

Keywords: small vessel disease, MRI, Fazekas

Introduction: One of the main signs of small vessel disease is cerebral white matter lesions (WML). The WML incidence detected by MRI images ranges from 5.5 to $100 \%$. WML is associated with cerebral blood flow, however, the causal relationship directly between the local decrease in cerebral blood flow and WML is unclear. The aim of this study was to assess the effect of the brachiocephalic arteries state on the number and severity of WML. 
Methods: 1171 patients with ischemic stroke during the last year were included in this study. These patients underwent brain MRI, brachiocephalic arteries duplex scanning, transcranial duplex scanning, $\mathrm{CT}$ angiography. We acquire data on the presence and severity of WML by Fazekas scale, the presence, and severity of microbleeds, the cerebral cortex local atrophy severity, assess the presence and severity of atherosclerotic lesions of the brachiocephalic arteries, linear and volumetric blood flow velocities, diameters of the carotid, vertebral arteries, basilar artery, and intracranial arteries. Statistical analysis was performed using SPSS Statistics version 25.0. ANOVA or Fisher's exact test was used to select potential WML predictors, depending on the dependent variable nature. Final WML predictors (with a Fazekas score of 2 or more) list were identified using binary logistic regression.

Results: The severity of periventricular WML was closely positively associated with the temporal lobes cortical atrophy degree $(\mathrm{p}<0.0005)$. The severity of deep and periventricular WML according to Fazekas was negatively associated with the end-diastolic and peak systolic velocities in all investigated extra- and intracranial arteries. In addition, in patients with both WML types, the diameters of most extra- and intracranial arteries were statistically significantly larger than in patients without WML. But no connection with the brachiocephalic arteries atherosclerotic lesions were found. According to the results of binary logistic regression, the main predictors of WML from the selected parameters were the diameters of different parts of the internal carotid arteries.

Discussion: The following logical chain is more likely by our data - large diameters of the brachiocephalic arteries lumen lead to a linear blood flow velocities decrease tendency, which, apparently, can lead (under certain conditions and in certain areas of the brain) to uneven hypoperfusion and, as a consequence, WML. Apparently, this process develops in parallel with atrophy of the cerebral cortex in the temporal lobes.

Conclusion: According to our data, WMLs are associated with large diameters and low blood flow velocities in the brachiocephalic arteries.

\section{1-P22}

ANGIOCENTRIC GLIOMA - A UNIQUE RADIOLOGICAL APPEARANCE

TIAGO LORGA ${ }^{1}$, Antonio $\mathrm{Cuco}^{2}$, Ines Freire ${ }^{1}$, Alexandra Santos ${ }^{2}$, Pedro Soares $^{1}$, Carlos Casimiro ${ }^{1}$

${ }^{1}$ Department of Neuroradiology, Hospital de Egas Moniz, CHLO, Lisbon, Portugal, ${ }^{2}$ Department of Neurosurgery, Hospital de Egas Moniz, CHLO, Lisbon, Portugal

Keywords: Angiocentric glioma, DNET, Oligodendroglioma, Ganglioglioma, SNC tumours, LEAT, Epilepsy

Introduction: Angiocentric glioma ( $\mathrm{AG}$ ) is a rare subtype of neuroepithelial CNS tumor, first described in 2005 and classified in 2016 as "other gliomas" and a WHO grade I tumor. AG predominantly occurs in pediatric patients and young adults, and most frequently manifests with drug-resistant epilepsy, being considered a long-term epilepsyassociated tumor (LEAT).

The diagnosis of AG relies on histology with the hallmark of angiocentric polarity with gliofibrillary acidic protein (GFAP) positive fusiform and bipolar astrocytic cells organized around blood vessels.

The lack of acquaintance with AG's radiological features impairs an accurate diagnosis. These tumors usually involve the cortex and subcortical white matter and present as a diffusely infiltrating lesion with illdefined borders. Characteristic MRI findings include a subtle T1hyperintense rim, T2/FLAIR hyperintensity, local mass effect with enlargement of cortical gyri and effacement of sulci. A T2/FLAIR hyperintense stalk-like extension to the ventricle is usually seen. Postcontrast enhancement and calcifications are typically absent. Focal cortical dysplasia can be present adjacent to the tumor.

Precise knowledge of imaging features is essential to reach an accurate diagnosis and to distinguish AG from other LEATs.

The aim of this work is to review the radiological characteristics of AG that may rule out differential diagnoses and allow prompt diagnosis.

Methods: We reviewed MRI studies of cases of LEATs and compared the radiological findings of AG with its main three differential diagnosis: dysembryoplastic neuroepithelial tumor (DNET), ganglioglioma and oligodendroglioma, regarding signal intensity, contrast enhancement, calcifications and hallmark features.

Results: AG presents a T1-hyperintense rim and a T2/FLAIR hyperintense stalk-like projection to the ventricle. DNET shows a cortical multicystic "bubbly" appearance and a minority can present enhancement and calcifications. Oligodendrogliomas and gangliogliomas typically show calcifications and enhancement, with the latter frequently located in the parahippocampal and temporooccipital regions and presenting cysts and an enhancing nodule.

Discussion: AG holds a unique radiological appearance; therefore, diagnosis can be strongly suggested prior to surgery in the context of a predominantly cortical lesion with a T1-hyperintense rim and a T2/FLAIR hyperintense stalk-like extension to the ventricle.

DNET, ganglioglioma and oligodendroglioma share radiological features with $A G$ and should be considered differential diagnosis. The recognition of AG advocates a favorable prognosis with very low mortality and incidence of long-term disability. Gross total resection is the treatment of choice and allows excellent results.

Conclusion: It is critical to be acquainted with AG imaging findings in order to achieve a correct diagnosis and proper management.

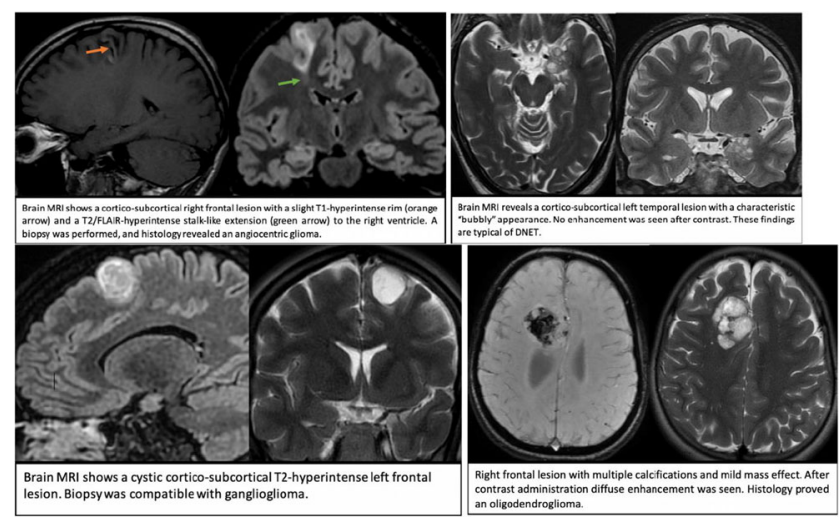

1-P23

NEUROIMAGING FINDINGS OF HYPERGLYCEMIC HYPEROSMOLAR STATE

INES SANTOS FREIRE ${ }^{1}$, Tiago Lorga ${ }^{1}$, Isabel Carmo ${ }^{2}$, Joana Barata Tavares $^{1}$, Gabriel Branco ${ }^{1}$, Carlos Casimiro ${ }^{1}$

${ }^{1}$ Neuroimaging Department, Centro Hospitalar Lisboa Ocidental Hospital de Egas Moniz, Lisbon, Portugal, ${ }^{2}$ Neurology Department, Centro Hospitalar Lisboa Ocidental - Hospital de Egas Moniz, Lisbon, Portugal 
Keywords: hyperosmolar hyperglycemic state, hemichoreahemiballismus, seizures

Introduction: Optimal brain function requires a continuous supply of glucose from the blood, as neurons demand an enormous amount of energy and depend on glucose as a source. The hyperosmolar hyperglycemic state (HHS) is a consequence of decompensated diabetes, mostly type 2 diabetes, with high mortality. Corresponds to deficient insulin function, yet with enough insulin levels to prevent the production of ketone bodies, along with an increase in counterregulatory hormones. Hyperglycemia and hyperosmolarity are responsible for neurological symptoms that include movement disorders, seizures and coma. Unlike diabetic ketoacidosis, which stands on other extreme of the spectrum of decompensated diabetes, well-established neuroimaging findings are only described in patients with HHS presenting with hemichoreahemiballismus (HC-HB) or seizures.

Methods: The authors present three cases of patients admitted to the emergency room with HHS, two of them with $\mathrm{HC}-\mathrm{HB}$ and the other with seizures. After bibliographic review, the authors focused on the classic neuroimaging findings of these conditions on CT and MRI.

Results: The first two cases correspond to patients with movement disorders in the context of hyperglycemia. The first patient was admitted with generalized choreic movements (fig. 1), while the second patient had abnormal left movements (fig. 2). Then, we present the neuroimaging findings in a patient with abnormal blood glucose levels and seizures (fig. 3), which correlate with the location of the ictal focus on EEG studies.

Discussion: In patients with $\mathrm{HC}-\mathrm{HB}$, NCCT may demonstrate hyperdensity in the striatal region contralateral to the symptomatic side. Changes can sometimes be bilateral and asymmetrical. On MRI, the most consistent finding is the respective hyperintensity on $\mathrm{T} 1$; on $\mathrm{T} 2$ it is variable but generally hypointense. Post-convulsive findings in the context of hyperglycemia are atypical with subcortical regions of hypointensity on T2/FLAIR in addition to other more recognized changes. There may be predilection for the occipital lobes. Imaging findings of both conditions gradually resolve after hyperglycemia correction.

Conclusion: Neuroimaging can play a vital role in early diagnosis and treatment of HHS, given its typical imaging appearance.

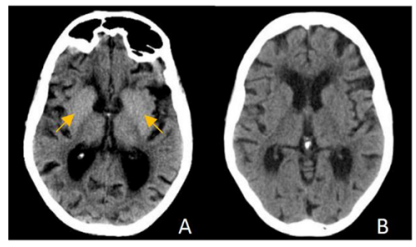

Figure 1: Non-contrast CT demonstrating spontaneous hyperdensity in the corpus striatum bilaterally (A, arrows), which was absent in a previous examination 1 year ago (B).

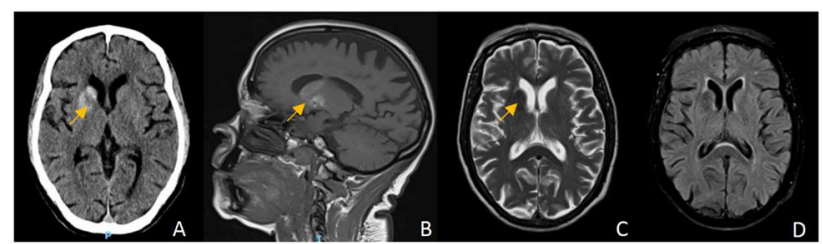

Figure 2: Non-contrast CT with spontaneous hyperdensity partially involving the right striatum (A, arrow). The respective MRI study demonstrates a corresponding hyperintensity on T1 (B) and hypointensity on T2/FLAIR ( $C$ and D). (Image courtesy of the Neuroimaging Department - Hospital Prof. Dr. Fernando Fonseca)

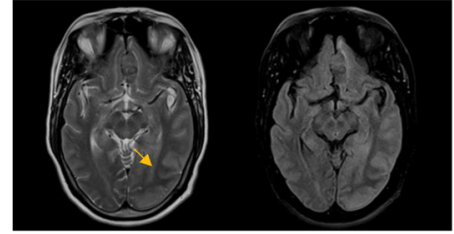

Figure 3: MRI revealed left occipital cortical T2/FLAIR hyperintensity extending to the parietal and temporal regions, with some swelling. There was hypointensity on $\mathrm{T} 2$ of the respective left subcortical white matter. Incidentally, an interhemispheric frontal meningioma is identified.

(Image courtesy of the Neuroimaging Department - Hospital Prof. Dr. Fernando Fonseca)

\section{1-P24}

ATYPICAL CASE OF CHOROID PLEXITIS AND EPENDYMITIS IN A HIV-POSITIVE PATIENT WITH AN UNDERLYING PRIMARY CNS LYMPHOMA - A CASE REPORT

MARIA INES SIMOES DA SILVA PRISCO ${ }^{1}$, Miguel Baptista $^{1}$, Sonia Rocha $^{2}$, Pedro Bem Gomes ${ }^{1}$, Fatima Hierro ${ }^{1}$, Ana Mafalda Fontes Pinto dos Reis ${ }^{1}$

1 Department of Neuroradiology; Unidade Local de Saude de Matosinhos, Portugal, ${ }^{2}$ Department of Infecciology, Unidade Local de Saude de Matosinhos, Portugal

Keywords: HIV, infection, lymphoma

Introduction: Although it is a rare primary tumor, Primary CNS lymphoma is common in HIV-infected patients, with some prevalence reports of $10 \%$ in autopsy studies and almost always associated with EBV infection.

Methods: We present a case of a 56 years-old caucasian male presenting to our hospital with a subacute onset of fever, confusion and 2-month weight loss. He had also history of perianal spinocelular carcinoma, bilateral temporal lobe epilepsy as well as a previous hospitalization in 2020 for CMV reactivation. He was submitted to a brain CT, MRI, lumbar puncture and laboratory tests.

Results: Brain CT showed an extensive subcortical and periventricular hipodensity and ventricular asymmetry that was posteriorly characterized by brain MRI; in concordance with CT, there was and extensive white matter and periventricular signal abnormality as well as a heterogeneous contrast enhancement of lateral and IV ventricle's ependyma and choroid plexus, suggesting choroid plexitis and ependymitis. There were no parenchymal lesions. It was performed a whole-body CT, which was negative, and he was also tested for infectious causes. Although CSF PCR for CMV was negative and undetectable HIV viral load, serum PCR was positive. It was initiated empiric antibiotic and antiviral treatment as well as anti-epileptics without any improvement. In the context of clinical worsening, it was performed a new lumbar puncture and CSF was then positive for EBV and immunophenotyping was positive for diffuse large B cell lymphoma.

Discussion: Our main goal is to analyze the radiological characteristics and the features of this case. We report a challenging, atypical case of an HIV-associated lymphoproliferative disorder with unusual morphologic features of choroid plexitis and ependymitis as a confounding diagnosis factor. Because of the positivity for CMV infection, he was diagnosed with CNS infection most likely relating the diagnosis with this opportunistic agent. It was only because of the clinical deterioration that it was performed a more detailed study, coming to conclusion that it was a lymphoproliferative disorder. 
Conclusion: Although it is a rare primary tumor, HIV-related primary lymphoma is a well-known entity, usually presenting as a rim enhancing lesion. However, differential diagnosis with opportunistic infections especially in a patient presenting with fever and radiological signs of choroid plexitis and ependymitis can make the diagnosis a challenging task, placing the infectious cause as main differential diagnosis. This case illustrates the complexity and alerts the neuroradiologist for the variety of radiological presentations of HIV-related conditions.
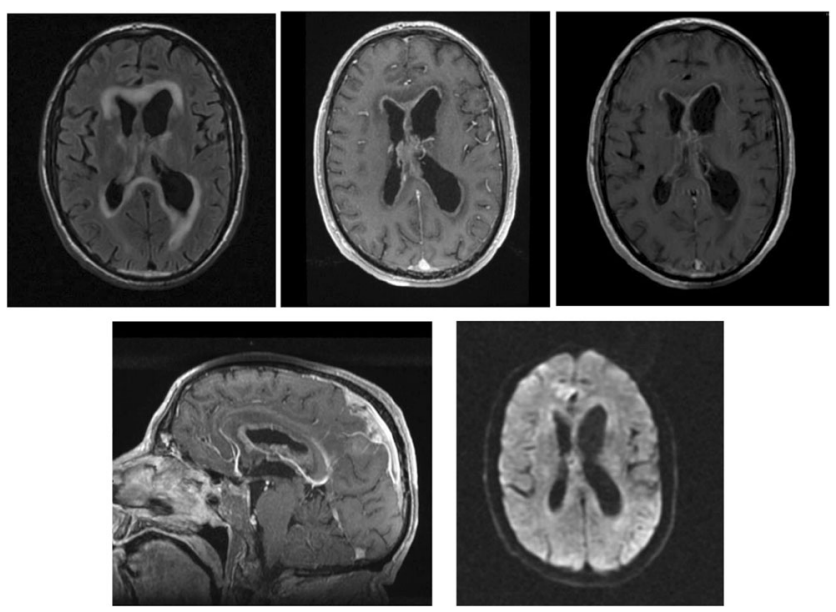

\section{1-P25}

T1W/FLAIR RATIO AS AN ALTERNATIVE FOR T1W/T2W RATIO TO MEASURE MYELIN CONTENT IN MS

\section{SARAH CAPPELLE $^{1}$, Deborah Pareto ${ }^{2}$}

${ }^{1}$ Department of Radiology, University Hospitals Leuven, Leuven, Belgium, ${ }^{2}$ Unitat de Ressonancia Magnetica (Servei de Radiologia), Hospital universitari Vall d'Hebron, Universitat Autònoma de Barcelona, Barcelona, Spain

\section{Keywords: Multiple Sclerosis, MRI, T1w/FLAIR ratio}

Introduction \& Purpose: Multiple Sclerosis (MS) is a demyelinating disease with an incidence of 2,8 million people worldwide. T1/T2 w ratio has been suggested as an alternative for MTR (magnetization transfer ratio) to measure quantitative myelination with good results. ${ }^{1}$ As nowadays, lesion detection in MS is evaluated on FLAIR images, possibly T1/ FLAIR ratios will be of more value in large studies and clinical routine. We want to evaluate the feasibility of T1/FLAIR ratio to serve as a proxy for myelin content in MS patients.

Methods: 227 Relapsing Remitting MS patients were scanned on 5 different $3 \mathrm{~T}$ scanners with the same protocol including 3D T1w, 2D T2w, 3D FLAIR images and MTI. ${ }^{2}$ Image analysis workflow can be seen in figure 1. The between-subject variation in NAWM in ratios with and without calibration was measured using the coefficient of variation $(\mathrm{CoV})$. (see Figure 2) Linear regression analysis of T1w/FLAIR ratio, T1w/T2w ratio and MTR in NAWM was performed corrected for age and gender in Analyse-it@.

Results: Figure 3 shows an example of a T1w/FLAIR ratio image on the left and a T1w/T2w ratio on the right. In Figure 4, we showed that $\mathrm{CoV}$ was reduced of the calibrated T1w/T2 $\mathrm{w}$ and $\mathrm{T} 1 \mathrm{w} / \mathrm{FLAIR}$ ratios compared to the non-calibrated ratios. Figure 4 shows a significant correlation of
$\mathrm{T} 1 \mathrm{w} / \mathrm{T} 2 \mathrm{w}$ ratio and T1w/FLAIR ratio with $\mathrm{R} 2=0.081, \mathrm{p}<0,0001$. Figure 5 shows a significant correlation of T1w/FLAIR ratio and MTR with $\mathrm{R} 2=0.153, \mathrm{p}<0,0001$.

Discussion \& Conclusion: Bias and intensity variation are widely present between different scanners, between subjects and within subjects over time in T1w, T2w and FLAIR images. Therefore, calibration of T1w/T2w and T1w/FLAIR ratio maps is absolutely necessary. FLAIR images could be used as an alternative for T2w images in the future to calculate a T1w/ FLAIR ratio. Although MTR and T1w/FLAIR ratio are driven by myelin integrity, they are not measuring exactly the same pathological substrate.

\section{References}

[1] Pareto et al. Ratio of T1-weighted to T2-weighted signal intensity as a measure of tissue integrity: comparison with magnetization transfer ratio in patients with multiple sclerosis. Am J Neuroradiol 2020

[2] Smets I et al. Quantitative MRI phenotypes capture biological heterogeneity in multiple sclerosis patients. Sci Rep. (2021) 11:1573
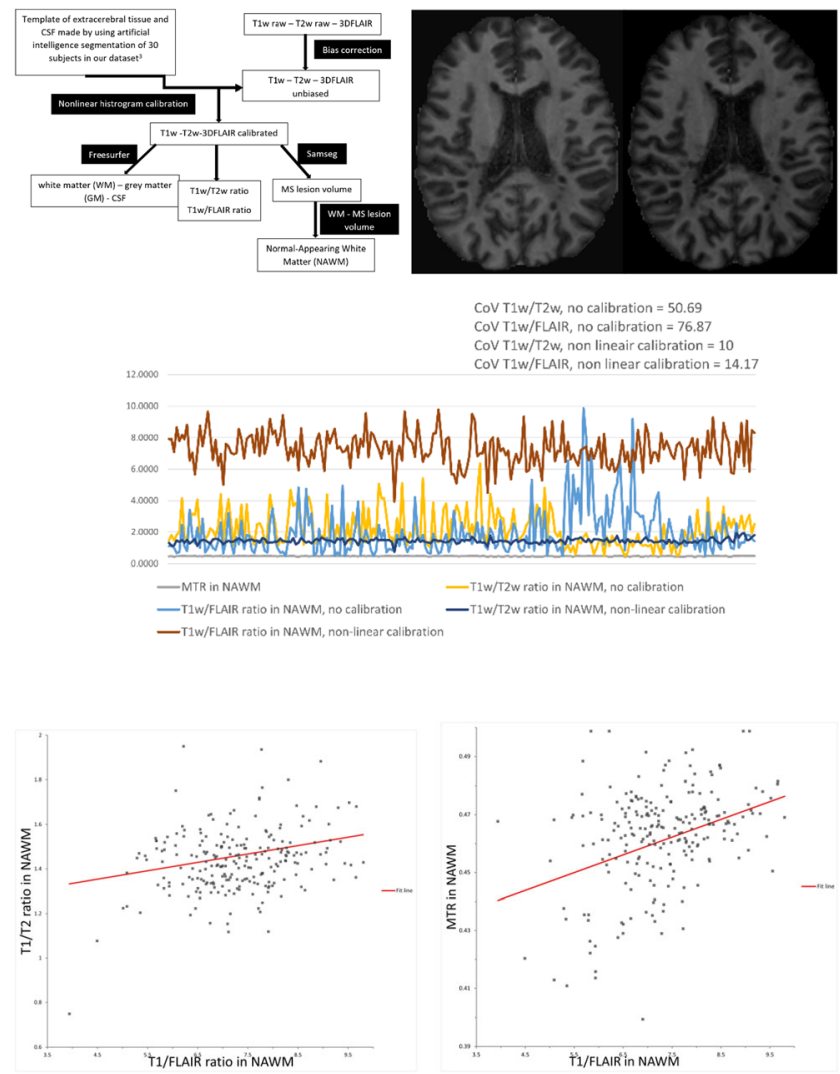

1-P26

THE ROLE OF BAYESIAN ESTIMATION ALGORITHM IN PREDICTING THE MOLECULAR STATUS OF GLIOMAS USING PREOPERATIVE DYNAMIC SUSCEPTIBILITY CONTRAST PERFUSION IMAGING

$\underline{\text { JAKOB MEGLIC }}^{1}$, Ajda Lisec $^{1}$, Katarina Surlan Popovic ${ }^{2}$, Jernej Avsenik $^{2}$

${ }^{1}$ University of Ljubljana, Ljubljana, Slovenia, ${ }^{2}$ Institute of Radiology, University Medical Centre Ljubljana, Ljubljana, Slovenia

Keywords: perfusion imaging, DSC-MRI, glioma, isocitrate dehydrogenase, Bayesian method 
Introduction: Dynamic susceptibility contrast (DSC)-MRI is a promising tool in preoperative assessment of gliomas with leakage corrected rCBV values typically considered a useful predictor of the isocitrate dehydrogenase (IDH) mutation status. Recently, a new fully adaptive and delay-insensitive Bayesian estimation of perfusion parameters was developed, outperforming the traditionally used delay-sensitive SVD algorithms in assessing the true perfusion parameter values in phantom experiments. Recent papers also suggest it is superior in predicting the final infarct size in acute ischemic stroke and may allow the use of reduced contrast dose in MRI perfusion studies. The aim of our study was to evaluate the performance of different perfusion calculation methods in predicting IDH status of gliomas.

Methods: Preoperative DSC-MRI studies of 60 glioma patients with grade II - IV tumors were retrospectively reviewed. MTT and CBF maps were calculated using both block-circulant SVD (cSVD) as well as Bayesian method. Additionally, leakage corrected rCBV and K2 maps were derived from the raw perfusion data. Region of interest (ROI) analysis was performed within the highest perfusion areas (hot spot) and in the FLAIR hyperintense tumor region. The difference between Bayesian method and cSVD derived MTT and CBF was analyzed, and the perfusion parameters were compared between IDH1 molecular status groups. Areas under the receiver operator curves (AUC) were used to compare the ability of different perfusion metrics in predicting IDH1 mutation status.

Results: IDH1 mutation was present in 9 patients and absent in 51. Hot spot $\mathrm{rCBV}$ values differed significantly between IDH1 groups $(\mathrm{p}=$ 0,034 ). Bayesian estimation and cSVD derived perfusion parameters (MTT and CBF) differed significantly in all ROIs with the exception of MTT in FLAIR hyperintense tumor region $(p=0,098)$. Hot spot rCBV performed best in predicting IDH1 mutation status (AUC 0,$723 ; 95 \% \mathrm{CI}$ $0,513-0,933)$, however it was not significantly better than Bayesian estimation of MTT in FLAIR hyperintense tumor region (AUC 0,703; $95 \%$ CI $0,532-0,874)$.

Discussion: Leakage corrected rCBV values proved to be the most efficient predictor of glioma genomics despite the relatively small sample size of IDH1 mutated tumors and this is consistent with previous research. However, our results suggest that MTT values calculated by recently developed Bayesian deconvolution algorithm may be similarly effective. The use of novel computational techniques may be advantageous in preoperative assessment of glioma patients.

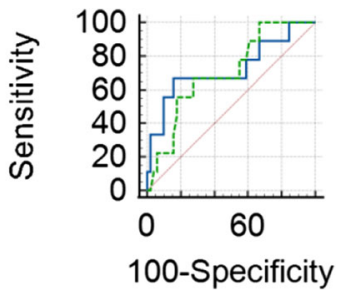

\section{1-P27}

SECONDARY DEGENERATION OF THE STRIATONIGRAL PATHWAY DUE TO IPSILATERAL CEREBRAL INFARCTION: A CASE REPORT

\section{$\underline{\text { EVANGELIA CHRISTODOULOU }}^{1}$, Michalis Mantatzis $^{1}$}

${ }^{1}$ Interventional Neuroradiology Unit, Department of Radiology/ Interventional Radiology, Democritus University of Thrace - Medical School/University General Hospital of Alexandroupolis, Alexandroupolis, Greece
Keywords: substantia nigra, striatum, cerebral infarction, degeneration, MRI

Introduction: Neuronal damage after focal cerebral infarctions can cause delayed neuropathologic changes to remote brain areas which are functionally and anatomically connected with the primary affected ischemic region. We present a case report of a patient with substantia nigra (SN) signal alterations due to basal ganglia ischemic stroke.

Materials \& Methods: A 56 year-old male patient admitted to our hospital due to left facial nerve palsy and left-sided hemiparesis with no past medical history. The patient underwent Magnetic Resonance Imaging (MRI) 8 days after the initialization of his symptoms.

Results: MRI scan was performed at 1,5 Tesla MR Unit. Hyperintense signal on T2W and FLAIR sequence at the level of the right lentiform and caudate nucleus was revealed with concomitant diffusion restriction. The findings were attributed to subacute ischemic stroke after occlusion of lateral lenticulostriate arteries. A high-signal-intensity lesion in the ipsilateral SN (more prominent in the pars reticulata than in the pars compacta) was also showed on $\mathrm{T} 2 \mathrm{~W}$ imaging. Diffusion restriction was also revealed. This condition was attributed to secondary degeneration of the SN after infarction in the striatum. After 12 days of patient's hospitalization left-sided hemiparesis was improved.

Discussion: This SN pathologic signal represents neuropathologic alterations of the striatonigral pathway. These degenerative changes are usually found after 7-14 days of the stroke onset. The mechanism of this result has been discussed mainly in animal experiments.

Conclusion: MRI allows to investigate degeneration in distal areas from the primary affected cerebral region (except from Wallerian degeneration). Radiologists should be aware of this condition in order not to be misdiagnosed as an additional ischemic area.

\section{1-P28}

A RETROSPECTIVE CROSS-SECTIONAL STUDY ON THE DARK SIDE OF TOMORROW: IMAGING OF DANCE FESTIVALRELATED MEDICAL EMERGENCIES IN NEURORADIOLOGY

DANAE COOLS ${ }^{1}$, Luc van den Hauwe ${ }^{1}$, Sven Dekeyzer ${ }^{1}$, Stephanie Vanden Bossche $^{1}$, Simon Nicolay ${ }^{1}$, Johan Van Goethem ${ }^{1}$, Annemiek Snoeckx ${ }^{1}$, Philippe Jorens ${ }^{2}$, Ingrid Baar ${ }^{2}$, Sabine Lemoyne ${ }^{3}$, Paul Parizel ${ }^{4,5}$

${ }^{1}$ Department of Radiology, Antwerp University Hospital, Antwerp (Edegem), Belgium, ${ }^{2}$ Department of Intensive Care, Antwerp University Hospital, Antwerp (Edegem), Belgium, ${ }^{3}$ Emergency Department, Antwerp University Hospital, Antwerp (Edegem), Belgium, ${ }^{4}$ Faculty of Medicine and Health Sciences, University of Antwerp, Antwerp, Belgium,

${ }^{5}$ Department of Radiology, Royal Perth Hospital and University of Western Australia Medical School, Perth, WA, Australia

Keywords: emergency, intoxication, trauma

Introduction: Dance festival-related medical emergencies represent a unique setting at the emergency department (ED). Our hospital is the major reference center during an annual large electronic dance music festival. The purpose of this study is to investigate the spectrum, causes and imaging findings of dance festival-related medical emergencies that present to the radiology department in the Neuroradiology section.

Methods: The study population consists of patients admitted to the ED because of emergencies related to attending a large electronic dance 
music festival. Our PACS was retrospectively searched for all urgent brain, head\&neck and spine CT and MRI studies performed in these patients referred by the ED during this large electronic dance music festival over a 10-year time period (2010-2019).

Results: 70 patients were scanned presenting with acute neurological symptoms at the ED: 52 men and 18 women with a median age of 28 years (range 18-56).

95 CT scans were performed in this patient group: 68 brain CT scans, 21 spine CT scans, 2 neck CT scans and 4 carotid CTA scans. 8/68 (11.8\%) brain CT scans were positive: 2 with posttraumatic acute intracranial hemorrhage, 3 with diffuse brain edema and 3 with maxillofacial fractures. $3 / 21(14.3 \%)$ spine CT scans were positive with posttraumatic fractures. $1 / 2(50.0 \%)$ neck CT scans was positive after a stabbing trauma. All carotid CTA scans were negative. 3 patients received an urgent spine MRI after their initial CT; 2/3 $(66.7 \%)$ with posttraumatic findings.

Recreational drugs and/or alcohol intoxication was the reason for admission in 35/70 (50.0\%) patients, trauma in 9/70 (12.9\%) and a combination of trauma, alcohol and/or drugs in 21/70 (30.0\%). 5/70 (7.1\%) had another or unclear cause.

Discussion: Most CT scans were negative $(83 / 95 ; 87.4 \%)$. Of the 12 positive scans, $9 / 12(75.0 \%)$ were trauma related, with or without intoxication. Of these, 1 presented with major posttraumatic intracranial hemorrhages, 1 with major cervical spine trauma and 1 with extensive stabbing wounds to the neck. 3/12 (25.0\%) patients - all female - presented with diffuse brain edema as a result of severe hyponatremia associated with drug intoxication (i.e. MDMA and cocaine), 2 of these patients died.

Conclusion: Neuro-imaging is common in patients presenting with dance festival-related emergencies at the ED. Most patients present with trauma and/or intoxication related symptoms, however major trauma is rare. An infrequent but devastating complication of drug intoxication is diffuse brain edema caused by severe hyponatremia, MDMA was involved in all cases.

\section{1-P29}

COMPARISON OF BLOOD-BRAIN BARRIER PERMEABILITY IN PERIMESENCEPHALIC AND ANEURYSMAL SUBARACHNOID HEMORRHAGE

JOAO PEDRO FREITAS GONCALVES ${ }^{1,2}$, Goncalo Gama Lobo ${ }^{1}$, Patricia Canhao $^{3}$, Isabel Fragata ${ }^{1,4}$

${ }^{1}$ Neuroradiology Department, Hospital Sao Jose, Centro Hospitalar Lisboa Central, Lisbon, Portugal, ${ }^{2}$ Neuroradiology Department, Hospital Dr. Nelio Mendonca, SESARAM E.P.E., Funchal, Portugal, ${ }^{3}$ Department of Neurosciences and Mental Health, Neurology, Faculty of Medicine, Hospital de Santa Maria, CHLN, University of Lisbon, Lisbon, Portugal, ${ }^{4}$ NOVA Medical School, Lisbon, Portugal

Keywords: perimesencephalic hemorrhage, subarachnoid hemorrhage, permeability

Introduction: Disruption of the blood brain barrier (BBB) is known to occur in aneurysmal subarachnoid hemorrhage (aSAH). However, less is known about the BBB changes in perimesencephalic hemorrhage $(\mathrm{PMH})$. We aimed to study BBB permeability in $\mathrm{PMH}$ and compare it to aSAH patients.

Methods: From a prospective cohort of 80 patients with spontaneous $\mathrm{SAH}$, we selected $\mathrm{PMH}$ patients and aSAH patients matched for clinical grade at admission (World Federation of Neurosurgeons Scale I/II). Computed tomography (CT) perfusion was performed at $<72 \mathrm{~h}$ and/or at 8-10 days. BBB permeability parameters Ktrans (volume transfer constant from blood plasma to extravascular extracellular space), Kep (washout rate constant of contrast agent from extravascular extracellular space to intravascular space), VE (extravascular extracellular space volume per unit of tissue volume), and VP (plasmatic volume per unit of tissue volume), were calculated from CT perfusion with Olea Sphere software. Mean values were compared using non-parametric tests.

Results: Fifteen PMH patients and 39 aSAH patients were included. At $<$ $72 \mathrm{~h}$ and 8-10 days, no significant differences in BBB permeability parameters were found between $\mathrm{PMH}$ and aSAH patients. There was a nonsignificant trend for increased Ktrans at 8-10 days in $\mathrm{PMH}$ patients when compared to aSAH patients $(0,21[0,13-0,30]$ vs. $0,14[0,11-0,18]$, $\mathrm{p}=0.056$ ), which might relate to higher $\mathrm{CBF}$ values in $\mathrm{PMH}$.

Conclusion: BBB permeability parameters were not significantly different between PMH and aSAH patients. Although PMH has a different etiology and clinical course, it may share pathophysiological mechanisms with aSAH, such as BBB dysfunction.

\section{1-P30}

BARRIERS FOR ACADEMIC ACTIVITY AND FACTORS INFLUENCING NEURORADIOLOGY SUBSPECIALTY SELECTION AMONGST RADIOLOGY TRAINEES

MARIA MERCEDES SERRA ${ }^{1}$, Sevcan Turk ${ }^{2}$, Joanna Marie Dy Choa$\mathrm{Go}^{3}$, Susan Shelmerdine, Eduardo Estades ${ }^{5}$, Ashlesha Satish Udare ${ }^{6}$, Monica Arzanauskaite ${ }^{7,8^{*}}$, Federica Vernuccio ${ }^{9^{*}}$

${ }^{1}$ MSc Program in Bioinformatics and Biostatistics, Universitat de Barcelona \& Universitat Oberta de Catalunya (UOC), Barcelona, Spain, ${ }^{2}$ University of Michigan, Radiology Department, Ann Arbor, USA, ${ }^{3}$ Radiology Department, Department of Radiology, The Doctors' Hospital, Inc.-Bacolod City, Negros Occidental, Philippines, ${ }^{4}$ Department of Clinical Radiology, Great Ormond Street Hospital, London, UK, ${ }^{5}$ Neuroradiology department, George Washington University, Washington DC, USA, ${ }^{6}$ Department of Radiology, Thomas Jefferson University Hospital, Philadelphia, USA, ${ }^{7}$ Radiology and Imaging Department, Liverpool Heart and Chest Hospital. Liverpool, UK, ${ }^{8}$ Cardiovascular Research Center-ICCC, Hospital de la Santa Creu i Sant Pau, IIB-Sant Pau, Barcelona, Spain, ${ }^{9}$ Section of Radiology, Department of Biomedicine, Neuroscience and Advanced Diagnostics (BIND), University of Palermo. Palermo, Italy

*These two authors equally contributed and share last authorship

\section{Keywords: Education, Academics, Neuroradiology, Training}

Introduction: Taking into account radiology trainees will lead development and progress of the field in following years, the main purpose of our work was to understand barriers towards academic activity within this group. As secondary goal in this work, we investigated factors influencing neuroradiology subspecialty selection.

Methods: A 35-question survey was distributed worldwide via social media and radiological societies diffusion to radiology trainees. A subgroup of surveyed interested in neuroradiology was selected. Analysis was conducted in R (R Core Team, 2020). Chi and Fisher's exact tests were used accordingly to evaluate factor associations and Wilcoxon ranksum test to evaluate differences in ordinal variable distributions. Significant alpha was set at the level of 0.05 .

Results: A total of 892 trainees participated in the survey. The most popular area of interest was abdominal radiology $(41 \%)$, followed by neuroradiology $(32 \%)$. Neuroradiology subgroup representation varied 
throughout different continents - Africa 31\% (12/39), Asia 40\% (77/191), Europe 29\%, (146/506), North America 24\% (22/92), South America $45 \%(27 / 60)$, Oceania $25 \%$ (1/4). We found no significant association between gender or age groups and neuroradiology interest. Most participants interested in neuroradiology showed two other areas of interest; we found a significant association between neuroradiology and head and neck interest. Other main associated interests followed the general group preferences. We found a significant association between neuroradiology interest and interest in pursuing a research fellowship. Having allocated research time was directly associated with the history of at least one conference presentation and at least one article publication. Lack of time, research, and teaching mentorship and lack of support from senior faculties were identified as main barriers for research and teaching activity.

Discussion: Though the survey provided us with a good overall global sample, Europe is the main represented continent and some regions are underrepresented. Neuroradiology trainees were more interested in performing a research fellowship than the general survey population, but we did not find differences in current research or teaching activity. Lack of time, mentorship, and support from senior faculty were identified as the main barriers.

Conclusion: Senior neuroradiologists should take advantage of the popularity of this subspecialty and mentor younger generations into teaching and research due to their interest in academic training. In order to contribute to the continuous academic progress of the field, radiology programs should also take into account the direct effect of allocated research and teaching time in academic production.
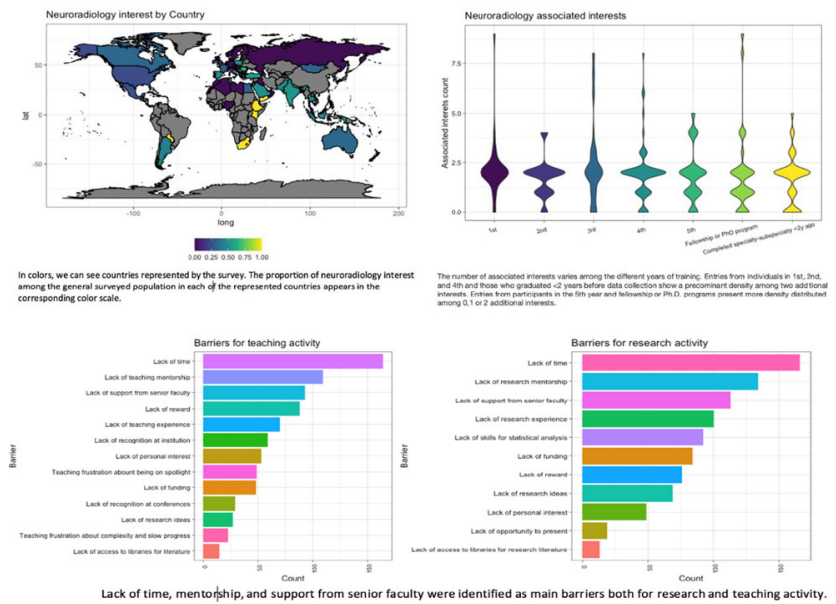

\section{1-P31}

MRI FINDINGS AND CLINICAL DATA IN CEREBRAL VASCULITIS: CONSISTENCY IN EVALUATION OF BRAIN AND VASCULAR CHANGES, CONNECTION WITH CLINICAL DATA, ROLE OF THERAPEUTIC DECISION

KINGA POZSAR ${ }^{1}$, Diana Solymosi ${ }^{1}$, Peter Varallyay ${ }^{1}$, Tunde Mangel ${ }^{1}$, Agnes Machovitsch ${ }^{1}$, Gabor Szudi ${ }^{1}$, Adam Kreiss ${ }^{1}$, Eszter Gerencser ${ }^{1}$, Orsolya Szalmas ${ }^{1}$, Aniko Papp ${ }^{1}$, Danuta Szirmai ${ }^{1}$, Claudia Futo ${ }^{1}$, Ahita Frendl ${ }^{1}$

${ }^{1}$ National Institute of Clinical Neurosciences (OMIII), Budapest, Hungary

Keywords: cerebral vasculitis, consistency in evaluation, clinical data, therapy
Introduction: An essential part of making the diagnosis of cerebral vasculitis is the proper evaluation of brain examinations. This work presents the accuracy of MRI diagnostics and its role in the therapy.

Materials, methods: We retrospectively reviewed images of 38 patients from a database of patients with suspected vasculitis without the knowledge of detailed clinical data. The work involved three radiologists experienced in neuroradiology, and the evaluation was performed independently. We judged parenchymal changes, stenosis of the medium sized arteries, and vessel wall enhancement. Taking the factors into account, we judged whether the presence of cerebral vasculitis arose. We compared our opinions with each other and with the clinical data.

Results: In case of 19 patients, we all agreed that vasculitis did not occur, as typical vascular wall enhancement was not detected. Non-specific inflammatory laboratory changes were confirmed in 10 patients. 18 patients were not treated. In case of 1 patient immunotherapy was initiated based on clinical data.

In case of 5 patients all observers agreed on vasculitis. In this group clear, concentric enhancement indicative of vasculitis was found in all patients. All patients had laboratory changes showing inflammation. All received therapy.

In case of 4 patients all observersagreed on small vessel vasculitis based on progressive white matter lesions. In 2 patients, based on the medical history, it was highly probable that the diagnosis of small vessel vasculitis could be made; 1 patient had vasculitis by histology; 1 patient had cerebral amyloid angiopathy by histology.

In 10 patients, the observers' opinions differed. Although they all agreed on parenchymal and vascular changes, there were differences in the assessment of vessel wall enhancement. Based on inflammatory disorders and history, 5 patients received therapy. 5 patients did not receive therapy due to clinical data.

Discussion \& Summary: Based on our results, it can be stated that with careful assessment of vascular wall enhancements and parenchymal changes in a significant number of cases the diagnosis of vasculitis can be consistent among the observers and can be made consistent with clinical data. In this case the therapeutic decision is clear. In a smaller proportion of cases, the MR image is uncertain. In this situation the therapeutic decision depends on the clinical data.

All in all, establishing the final diagnosis of cerebral vasculitis requires an interdisciplinary approach, there is a need of clinical consultations, evaluation of several observers and follow up examinations.

\section{1-P32}

WALLENBERG SYNDROME: SMALL LESION - BIG CLINICAL PICTURE!

\section{$\underline{\text { SVJETLANA JEFIC }}^{1}$, Daliborka Maric $^{2}$, Dalibor Vranjes ${ }^{3}$}

${ }^{1}$ Polyclinic "Al-Tawil", Radiology Department, Sarajevo, Bosnia and Herzegovina, ${ }^{2}$ University Clinical Centre of the Republic of Srpska, Department of Radiology, Banja Luka, Bosnia and Herzegovina, ${ }^{3}$ University Clinical Centre of the Republic of Srpska, Ear, Throat and Nose Department, Banja Luka, Bosnia and Herzegovina

Keywords: Wallenberg syndrome, stroke, MR imaging

Introduction: Wallenberg syndrome (WS), known as lateral medullary (LM) syndrome is a result of infarction in the lateral part of the medulla oblongata. Clinical symptoms, sometimes very serious, can vary due to the abundance of the critical neurological structures in this region, whereas area of insult can be very small and subtle. Although the clinical triad of Horner syndrome, ipsilateral ataxia and contralateral hypalgesia is 
estimated as typical WS, it is not common. Usually WS is not complete or it can be nonspecifically presented so that the accurate diagnosis couldn't be possible without MR imaging.

Method: We present four instructive, comparatively analyzed cases with mostly non-specific clinical picture and a normal CT brain scan as an initially performed imaging test.

Case 1: A 63 yo man with history of arterial hypertension (HTA) and sudden onset of vertigo.

Neurological findings (NF): Slightly positive Romberg test.

MR findings: Small focal lesion in the right LM with high signal in the T2-weighted (T2W), fluid attenuation inversion recovery (FLAIR), diffusion weighted image (DWI) sequences, and low apparent diffusion coefficient (ADC)-signal, consistent with lateral medullary infarction.

Case 2: A 68 yo woman, HTA, sudden onset of headake, nausea and vomiting associated with vertigo, slurred speach, tingling around the mouth and chin.

NF: Without acute neurological deficit.

MR findings: In the left LM, in the region of inferior cerebellar peduncle, the pattern of changes suggestive of acute infarction.

Case 3: A 65 yo old man, HTA, headake, general weakness, sweating, fatigue, difficulty speaking and swallowing.

NF: Discrete motor dysphasia and Horner syndrome.

MR findings: In the right LM, the region of trigeminal nerve nucleus and spinal tract, discrete ribbon of signal changes indicating an acute infarction. MR angiography (MRA) showed dissection of the $\mathrm{C} 1$ segment of the right internal carotid artery (ICA).

Case 4: A 61 yo man, HTA, sudden onset of vertigo, headake, vomitus and general weakness.

NF: Horizontal nistagmus, both sides.

MR findings: Acute infarction of the right posterolateral medulla as well as inferior aspect of the right cerebellum.

Results: All four patients had good outcome including the one with carotid dissection whose recovery period was prolonged.

Conclusion: Non-specific clinical presentation of WS and its discrete radiological presentation in some cases, especially in combination with daily work overload, can cause a misleading diagnosis and therefore postponement of timely application of therapy. Familiarity with the issues of WS prevents neuroradiologists from making potential mistakes.

\section{1-P33}

A RETROSPECTIVE ANALYSIS OF MRI FINDINGS OF CENTRAL NERVOUS SYSTEM TUBERCULOSIS (CNS-TB) IN A SPANISH REFERENCE CENTER

YIKEN KARELYS NG WONG $^{1}$, Albert Pla $^{1}$, Cristina Auger ${ }^{1}$, Alex Rovira

${ }^{1}$ Neuroradiology, Hospital Universitari Vall d'Hebron, Barcelona, Spain

Keywords: CNS-TB, Tuberculosis, Cns Infection, Vasculitis

Objectives: To describe the main findings in MRI, the clinical and analytical characteristics and the outcome of central nervous system tuberculosis (CNS-TB) observed in our center.

Methods: A retrospective review of the medical records of patients with CNS-TB in a University Hospital of Barcelona, from 2007-2018, was performed. Microbiologically confirmed patients were included.

Results: We reviewed 30 cases, $70 \%$ were men, mean age $41.8 \pm 25.4$; $60 \%$ were immigrants. As main comorbidities: chronic kidney disease
(23\%), diabetes mellitus (20\%), chronic liver disease (13\%), alcoholism (10\%) and immunosuppression (43\%), mainly HIV. Predominant clinical findings at admission: fever (100\%), headache $(93.3 \%)$, irritability and meningeal signs (53.3\%), vomiting (30\%), neurological deficit and cranial nerve involvement (36.7\%) and seizures (30\%) The CSF culture was positive in $38 \%$ of cases. Leptomeningeal disease $(68 \%)$ and cerebral infarction (46\%) were the most frequent findings in MRI, followed by tuberculoma, hydrocephalus, cerebritis, neuritis and ventriculitis. The univariate analysis showed a significant association between chronic kidney disease with non-significant findings in cerebral MRI. The overall mortality was $23 \%, 16 \%$ directly attributable to the CNS-TB. The presence of leptomeningitis and cerebral infarction in MRI were predictors of poor prognosis ( $93 \%$ vs. $43 \%$, p: 0.013 ; and $71 \%$ vs. $21 \%$ p: 0.021 ). However, it was non-significant in multivariate analysis (leptomeningitis (OR 9.5 (95\% CI, 0.84-106.4)); cerebral infarction (OR 4.7 (95\% CI, 0.7 31.5)

Conclusions: The high mortality and neurological sequelae of TB-CNS were confirmed. Most of the patients have MRI pathological findings, the most frequent being leptomeningeal disease and cerebral infarctions. Both were associated with a poor prognosis in a univariate analysis, losing significance in a multivariate study, probably due to small sample size. Diagnosis and prior treatment remain crucial in TB-CNS. MRI can help stratify patients according to the risk of death or associated neurological complications.

\section{1-P34}

MR NEUROGRAPHIC IN OCCIPITAL NEURALGIA: ANATOMY AND IMAGING FINDINGS OF THE GREATER OCCIPITAL NERVE

JOAO VICTOR PINTO RODRIGUES ${ }^{1}$, Danielly Viana Monteiro Santos $^{1}$, Matheus Dorigatti Soldatelli ${ }^{1}$, Lazaro Luis Faria do Amaral ${ }^{1}$, Victor Hugo Marussi ${ }^{1}$

${ }^{1}$ Hospital Beneficencia Portuguesa de Sao Paulo, Sao Paulo, Brazil

Keywords: occipital, neuralgia, neurographic

Introduction: Headache is a major cause of disability, affecting more than $10 \%$ of the population, with $4 \%$ of adults having a chronic daily headache $(\mathrm{CDH})$. Chronic migraine and chronic tension-type headache (TTH) comprise the majority of $\mathrm{CDH}$. For occipitally located pain, a similar myriad of diagnoses exists. In unilateral cases, occipital neuralgia $(\mathrm{ON})$ could be a diagnosis; for bilateral cases, TTH would seem more appropriate.

Neuralgias are syndromes characterized by intermittent attacks of sharp and paroxysmal pain along the course of a nerve. In ON, greater occipital nerve (GON) compression can be considered one of the causes.

Objective \& Methods: This study aims to review the anatomy of the greater occipital nerve and the imaging findings in MRI Neurography in unilateral occipital neuralgia.

Discussion: The GON is a cutaneous nerve, the thickest in the body, that innervates the skin from the upper neck, up to the vertex of the scalp. The GON follows a predictable course through the posterior vertebral musculature, comprising three portions and two bends, giving ample opportunity for entrapment and compression.

It arises from the dorsal ramus of $\mathrm{C} 2$. The first portion (P1) runs a short oblique course towards the lower border of the obliquus capitis inferior muscle; the first bend (A1) occurs around this muscle. The second portion (P2) travels posteromedially and upwards between two muscular layers: semispinalis capitis (superficial layer) and obliquus capitis inferior and 
rectus capitis posterior major (deep layer) muscles. The second bend (A2) occurs as the GON crosses the semispinalis capitis, piercing through its fibers. The third and last portion (P3) travels superiorly between the dorsal aspect of the semispinalis capitis and the deep aspect of the trapezius. The GON perforates the trapezius aponeurosis to reach the subcutaneous layer near the nuchal line.

MR neurography is multiplanar nerve-selective imaging used to evaluate peripheral nerve anatomy and pathology. The nerve can be identified on coronal and sagittal thick-slab MIP images. Asymmetric hyperintensity and caliber alteration can be seen across the points of compression under the semispinalis capitis muscles and/or adjacent fascia, suggesting neuropathy in unilateral migraine cases.
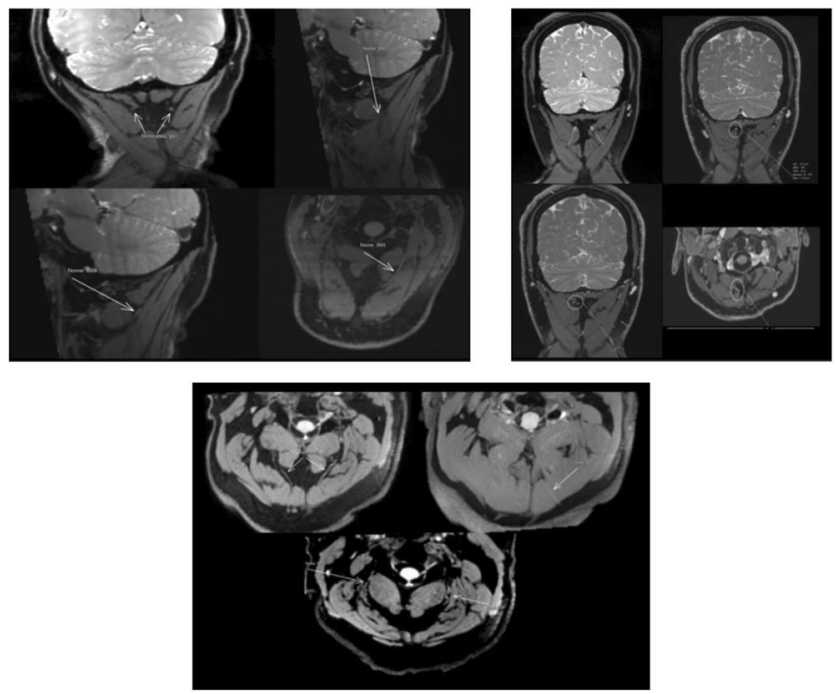

\section{1-P35}

A PRACTICAL FMRI PROTOCOL FOR ASSESSING MEMORY IN MESIAL-TEMPORAL EPILEPSY SURGERY CANDIDATES: DESCRIPTION OF ACTIVATION PATTERNS

PABLO NAVAL-BAUDIN ${ }^{1}$, Angels Camins-Simon ${ }^{1}$, Jacint SalaPadro $^{2}$, Aleix Rossello ${ }^{3}$, Estela Camara ${ }^{4}$, Lluis Fuentemilla ${ }^{4}$, David Cucurell $^{4}$, Inmaculada Rico Pons ${ }^{2}$, Merce Falip ${ }^{2}$, Alejandro FernandezCoello ${ }^{3}$

${ }^{1}$ Neuroradiology, Institut de Diagnostic per la Imatge, Hospital Universitari de Bellvitge, L'Hospitalet de Llobregat, Barcelona, Spain, ${ }^{2}$ Neurology, Hospital Universitari de Bellvitge, L'Hospitalet de Llobregat, Barcelona, Spain, ${ }^{3}$ Neurosurgery, Hospital Universitari de Bellvitge, L'Hospitalet de Llobregat, Barcelona, Spain, ${ }^{4}$ Cognition and Brain Plasticity Group, Bellvitge Biomedical Research Institute- IDIBELL

Keywords: fMRI, epilepsy, memory, surgical planning

Introduction: Memory loss is one of the main sequelae of medial temporal lobe resection, affecting approximately 25 - 33\% of patients. Language lateralization techniques such as Wada or language functional MRI (fMRI), have proven to predict language and memory outcomes to some degree.

Memory fMRI has been proposed, with good though varied results. However, there is no general established consensus regarding several aspects: 1) paradigms to apply, 2) patterns of normal activation, 3) relevant regions of interest.
We propose a memory fMRI protocol and analyze the different patterns of normal activation in healthy control subjects compared with patients candidate for medial-temporal lobe surgery.

Methods: We included 10 surgery candidates and six healthy controls. All subjects were submitted to the full memory fMRI protocol (3T Philips Ingenia S system with 32-channel head coil).

The three block-design paradigms included were verbal encoding, scene encoding and autobiographical memory. For verbal and scene encoding paradigms, nouns or pictures of scenes respectively were presented visually consecutively in blocks of 10 , mixed with periods of crosshair fixation. Participants had to memorize items for subsequent recall testing after the scan. For the autobiographical memory paradigm, patients had to recall 10 events from their own history.

fMRI analysis and data collection were performed by consensus by two neuroradiologists with specific subspecialized dedication to presurgical fMRI brain mapping. Philips Intellispace Portal BOLD pre-processing workflow was used.

Results: For the six healthy control subjects, autobiographical memory paradigm elicited robust hippocampal activations in all six subjects, scene encoding in five, and word encoding in three. Regarding lateralization, word-encoding presented the most lateralized hippocampal and parahippocampal activations, especially in anterior regions, while scene encoding presented more symmetric activations predominantly in hippocampal tail and posterior parahippocampal gyri.

Seven of the ten patients had a left-sided epileptogenic focus. These presented less left than right activations. The three right-sided foci patients presented more symmetrical activations.

\section{Discussion \& Conclusion:}

The three different paradigms elicited very different patterns of activation. Words had more anterior lateralized activations, scenes had more posterior symmetric activations, and autobiographical had anterior symmetric activations.

Preestablishing the patterns of activations for each of these paradigms is vital for understanding the significance of variations in specific patients and for interpreting their clinical application. Each of these paradigms provides complementary information on the distribution of different aspects of memory function. The post-surgical repercussion of the presence and patterns of distribution of activations should be studied prospectively.
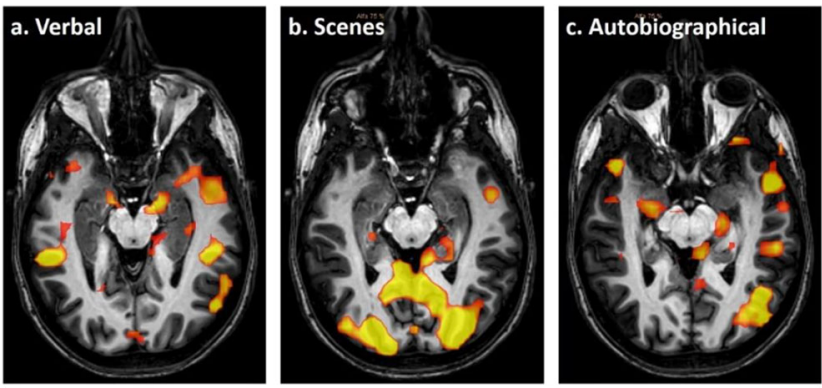

Figure. fMRI Activation maps overlaid on axial T1WI. Right part of the image represents left hemisphere and vice-versa. (a) Verbal encoding paradigm demonstrates robust bilateral amygdala and left hippocampal body activations. (b) Scene encoding demonstrates robust bilateral posterior hippocampal hippocampal body activations. (b) Scene encoding demonstrates robust bilateral posterior hippocampal
and posterior mesial temporal and lingula activations. (c) Autobiographical memory paradigm demonstrates robust right amygdala and left hippocampal body activations.

\section{1-P36}

USEFUL ASPECTS AND CLUES IN SUBTYPE DIFFERENTIATION OF PITUITARY MACROADENOMAS

RANGEL DE SOUSA COSTA ${ }^{1}$, Ferdinand Duenas Cabrera Filho ${ }^{1}$, Nina Ventura, Eduardo Jorge Lemos Neves Filho ${ }^{1}$, Ananda Altoe ${ }^{1}$ 
${ }^{1}$ Instituto Estadual do Cerebro Paulo Niemeyer, Rio de Janeiro, Brazil

Keywords: Hypophysis, Macroadenomas, MRI

Teaching Points: 1) Pituitary adenoma is the most common tumor of the sellar region in adults. They are classified based on hormonal secretion into functional and non-functional, and based on dimensions in macro (> $1 \mathrm{~cm})$ and microadenomas $(<1 \mathrm{~cm})$. There are no specific findings that differentiate histological subtypes, but some imaging features can be characteristic.

2) The objective of this pictorial essay is to propose magnetic resonance imaging (MRI) aspects that could differentiate some subtypes of macroadenomas.

3) Corticotroph macroadenomas usually present areas of microcystic degeneration. Giant prolactinomas tend to represent a more irregular lesion, often with invasion of adjacent structures and skull base. Somatotroph adenomas usually have a greater infrasellar than suprasellar extent, and is generally more hypointense in T2. Gonadotroph subtypes tend to be larger and more aggressive lesions, with areas of cystic degeneration. Clinical silent ones are those with no relevant hormone secretion but are positive for specific hormone on immunohistochemistry.

4) In terms of prognosis, clinically silent corticotroph adenomas have a worse prognosis, while somatotroph adenomas with a lower signal at T2 usually have a better prognosis.
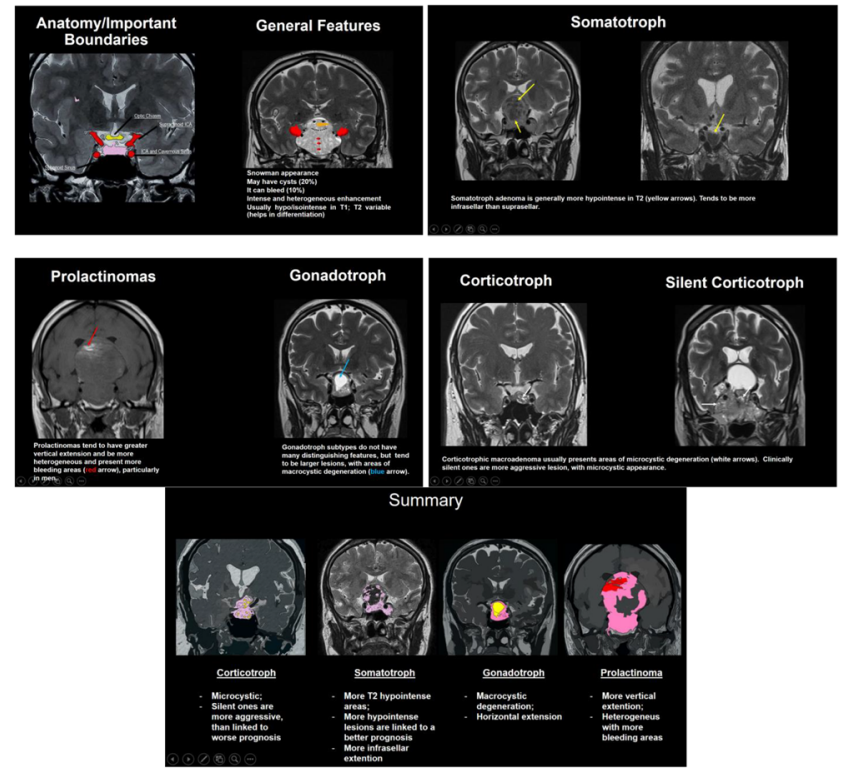

\section{1-P37}

WHERE DID IT CAME FROM? A BRAIN ABSCESS IN A PATIENT WITH HEREDITARY HEMORRHAGIC TELANGIECTASIA CLINICAL CASE

JOSE MANUEL SA DA SILVA ${ }^{1}$, Vasco Sousa Abreu ${ }^{1}$, Eduarda Maria Marinho Pinto $^{1}$, Sergio Sousa ${ }^{2}$, Goncalo Videira ${ }^{3}$, Joao Carvalho ${ }^{4}$, Joao Pedro Filipe ${ }^{1}$, Sofia Pina ${ }^{1}$

\footnotetext{
${ }^{1}$ Neuroradiology Department, University and Hospital Center of Porto, Porto, Portugal, ${ }^{2}$ Neurosurgery Department, University and Hospital Center of Porto, Porto, Portugal, ${ }^{3}$ Neurology Department, University and Hospital Center of Porto, Porto, Portugal, ${ }^{4}$ Radiology Department, University and Hospital Center of Porto, Porto, Portugal
}

Keywords: Hereditary hemorrhagic telangiectasia, Brain abscess, Pulmonary arteriovenous malformation

Introduction: Pulmonary arteriovenous malformation (PAVM) is a known complication of hereditary hemorrhagic telangiectasia (HHT). The right-to-left shunt leads to paradoxical embolism. Cerebral abscess rates are reported up to $9 \%$ in patients with HHT/PAVMs, probably resulting from septic emboli. De novo neurological deficits in a patient with HHT should always prompt neuroimaging in search of an abscess or stroke.

Clinical Case: A 48 yo female with prior history of hereditary hemorrhagic telangiectasia presented to the emergency department with new onset motor aphasia. On admission, she had an inaugural generalized tonic-clonic seizure. She partially recovered from the aphasia and was discharged home on anti-epileptic drugs but returned 10 days later because the aphasia progressively worsened. Besides emotional liability and acalculia, she had no other deficits nor clinical findings. Head CT showed a large frontal left subcortical hypodensity with ring enhancement and surrounding edema. Further investigation with MRI revealed a ringenhancing lesion with restricted diffusion on its center and the double rim sign_on SWI, all findings pointing to a cerebral abscess. She was started on IV antibiotics and underwent surgical drainage of the abscess (microbiological analysis was positive for Eikenella corrodens and Streptococcus intermedius, probably originating from dental caries), fully recovering from the aphasia several days after.

Once stable, further investigation with pulmonary CT disclosed 3 PAVMs in the right superior lobe. She then underwent angiography to embolize all PAVMs, with complete exclusion. At five months follow-up there were no complications nor signs of relapse.

Conclusion: HHT patients might have their disease undiagnosed before a severe (neurological or non-neurological) complication reveals it, but a patient with known HHT and new onset clinical findings cannot be discharged without thorough investigation excluding serious treatable conditions. For de novo neurological deficits, head CT must be regarded necessary, and MRI considered, always being mindful of the greater risk of stroke and cerebral abscess in these patients.
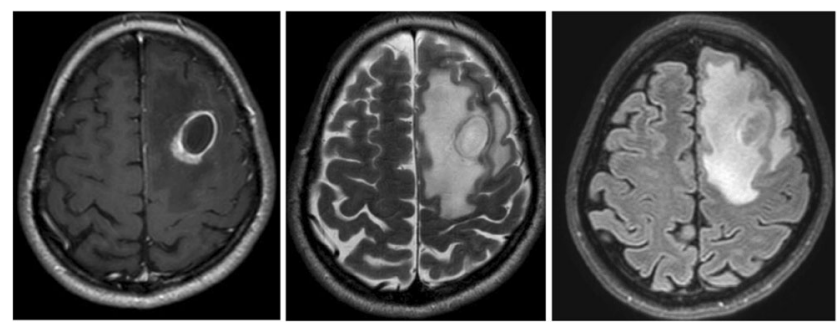

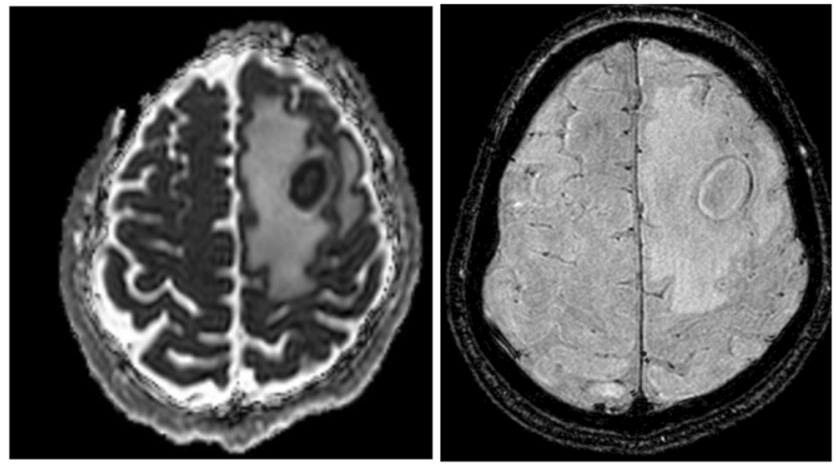

1-P38

MOYAMOYA DISEASE: FROM DIAGNOSIS TO TREATMENT

DANIELLY VIANA MONTEIRO SANTOS ${ }^{1}$, Marcela Aparecida Felipe $^{1}$, Juliana Frazatti Benez ${ }^{1}$, Tauy Pereira Morimoto ${ }^{1}$, Matheus Dorigatti Soldatelli ${ }^{1}$, Christiane Monteiro De Siqueira Campos ${ }^{1}$

${ }^{1}$ Hospital Beneficencia Portuguesa of Sao Paulo, Sao Paulo, Brazil

Keywords: moyamoya, diagnosis, treatment

Introduction: Moyamoya's disease is an idiopathic steno-occlusive entity caused by intimal hyperplasia and medial thinning, which mainly affects the termination of the internal carotid artery and the proximal anterior and middle cerebral arteries. As a result, there is the formation of a collateral mesh of small dilated arteries (the moyamoya vessels). Aneurysms can arise in the moyamoya vessels, being the main cause of hemorrhage.

It is more common in the East Asian populations and during childhood. It can be considered moyamoya disease when there is angiographic findings of moyamoya and no risk factor or moyamoya syndrome when it is associated with other conditions.

Based on its presentation into four: ischemic, hemorrhagic, epileptic, and "other." In children, ischemic stroke is the primary presentation while in adults the primary presentation is that of intracranial hemorrhage. The cause of death in most autopsy cases is intracerebral hemorrhage.

Objective \& Methods: This work aims to review the main findings of the pre and postoperative image of moyamoya disease and to discuss the main surgical techniques. For this, 16 patients with moyamoya disease from our service were retrospectively analyzed.

Discussion: Imaging methods are essential for the diagnosis and evaluation of complications of moyamoya disease, the main signs are exemplified in the images below.

The disease inevitably progresses if left untreated and the risk of recurrent stroke is extremely high with clinical treatment only. The goal of the surgery is to prevent the next episode by restoring adequate blood supply to the affected cortex through brain bypass surgery, which is revascularization between the extracranial vessel and the intracranial vessel or between the intracranial vessel and another intracranial vessel using a graft (superficial temporal artery). Older adults and children can undergo brain vessel revascularization surgery, and younger children can undergo indirect revascularization surgery because their vessels are very small and tend to fail if we do a direct bypass. Among the options of indirect revascularization, we have encephalomyosinangiosis (EMS), encephaloduroarteriosynangiosis (EDAS), and encephaloduroarteriosynangiosis (EDAMS). EMS surgery establishes a new vascularization, using the temporal muscle as a source of angiogenesis to the brain. EDAS surgery places the dissection superficial temporal artery in direct contact with the cortical surface, in an attempt to establish a new vascularization in the brain. EDAMS surgery is a combination of EDAS and EMS at the same time.
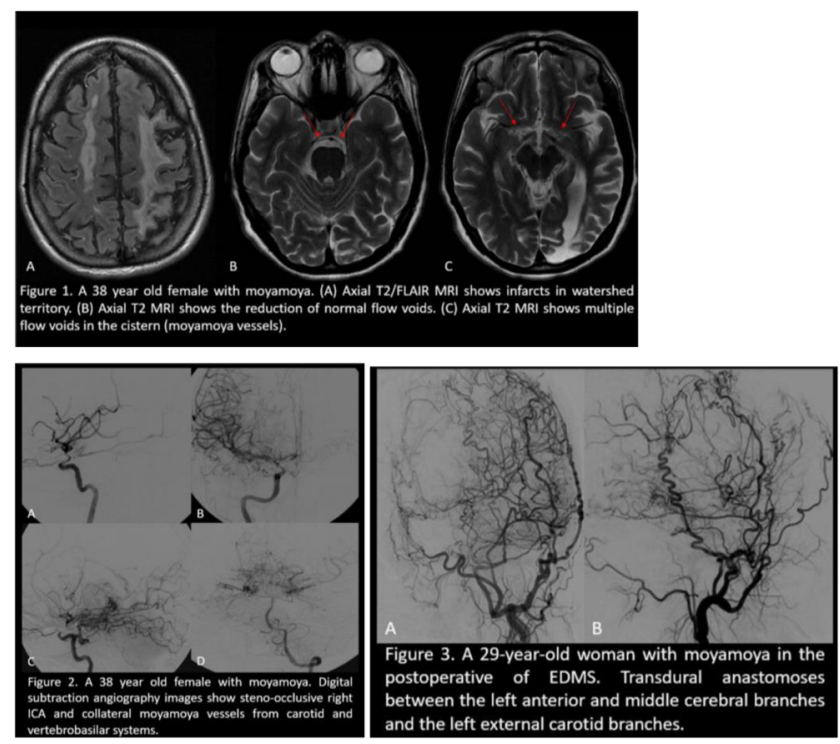

\section{1-P39}

SELLAR CYSTIC LESIONS: MRI CHECKLIST FOR RADIOLOGIST

EDUARDO JORGE LEMOS NEVES FILHO ${ }^{1}$, Ferdinand Duenas Cabrera Filho $^{1}$, Nina Ventura ${ }^{1}$, Rangel de Sousa Costa $^{1}$

${ }^{1}$ Radiology Department, Instituto Estadual do Cerebro Paulo Niemeyer, Rio de Janeiro, Brazil

Keywords: cystic sellar lesions, MRI checklist, differential diagnosis

Teaching Points: 1) Sellar's cysts are often challenging diagnosis by clinical and imaging exams. The pattern of clinical and imaging aspects are very similar between these lesions. The correct imaging evaluation is difficult, but essential to decide the best treatment.

2) The purpose of this pictorial essay is to suggest a checklist and a flow chart with the most important aspects found in the magnetic resonance imaging of the most common cystic lesions of sella turcica.

3) The lesions included in this study are: Rathke's cleft cyst, arachnoid cyst, craniopharyngioma, cystic pituitary adenoma.

4) Checklist of sellar cyst by magnetic resonance imaging: Arachnoid cyst: pattern of cerebrospinal fluid (hyperintense signal in FLAIR/T2, and best definition in another fluid sequences like CISS); no wall enhancement. Craniopharyngioma: Identify pituitary gland; identify calcification; lesion extends to pituitary stalk; irregular wall and/or nodule. Rathke's cleft cyst: Identify hypointense nodule in T2; thin enhancement of wall; midline cyst within the sella arising between the anterior and intermediate lobes of the pituitary. Cystic pituitary adenoma: level fluid/ fluid; septations; identify sellar diaphragm; irregular enhancement. 

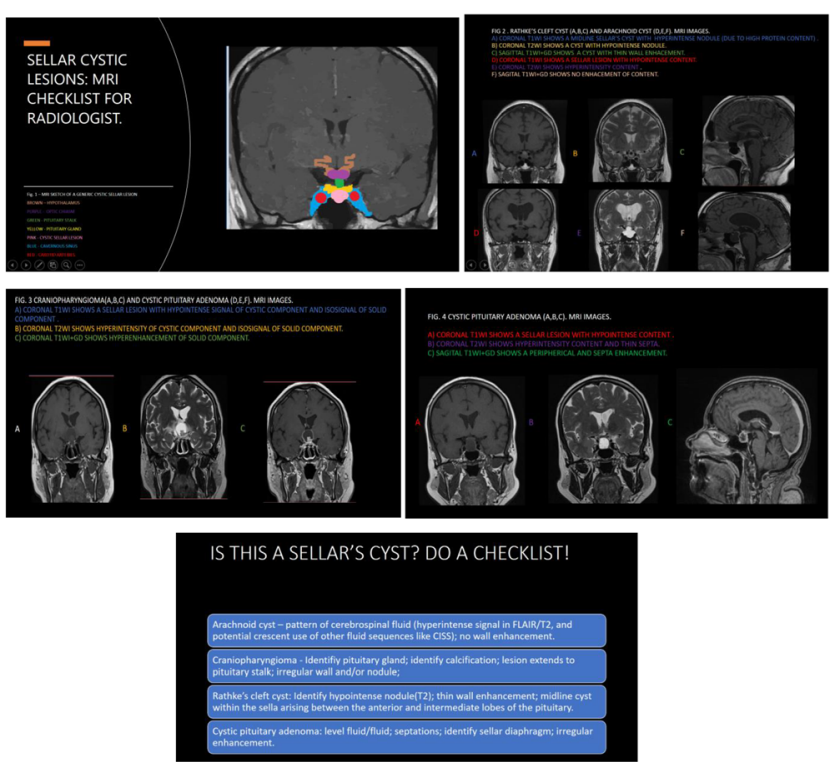

\section{1-P40}

THE ROLE OF CENTRAL VEIN SIGN IN THE DIFFERENTIAL DIAGNOSIS BETWEEN MULTIPLE SCLEROSIS AND FABRY DISEASE

MARIO TRANFA $^{1}$, Mario Tortora ${ }^{1}$, Giuseppe Pontillo ${ }^{1}$, Valentina Iuzzolino $^{2}$, Eleonora Riccio ${ }^{3}$, Vincenzo Brescia Morra ${ }^{2}$, Maria Petracca $^{2}$, Sirio Cocozza ${ }^{1}$, Arturo Brunetti ${ }^{1}$

${ }^{1}$ Department of Advanced Biomedical Sciences, University of Naples "Federico II", Naples, Italy, ${ }^{2}$ Department of Neurosciences and Reproductive and Odontostomatological Sciences, University of Naples "Federico II", Naples, Italy, ${ }^{3}$ Department of Public Health, Nephrology Unit, University of Naples "Federico II", Naples, Italy

Keywords: Multiple Sclerosis, Central Vein Sign, Fabry Disease

Background \& Purpose: In recent years Fabry Disease (FD) has been proposed as a possible Multiple Sclerosis (MS) mimicker, especially in the early stages of the disease when MRI presentation could share some similarities. Neuroradiological signs suggestive of MS, like callosal or infratentorial involvement, have been recommended as useful tools to differentiate between these two conditions. Nonetheless, achieving a proper diagnosis could still be challenging. We evaluated the incidence of an emerging neuroradiological sign highly linked to MS physiopathology, such as the Central Vein Sign (CVS), and its performance in differentiating between these two conditions.

Methods: Brain MRI scans of 57 FD patients and 36 MS patients with a disease duration less than 5 years were evaluated in consensus by two neuroradiologists. After assessing a Fazekas Score (FS) for all patients, cases with FS equal to 0 were removed from further analyses. For all patients, we obtained fusion images from FLAIR T2-weighted and SPGR volumes, and then we evaluated the presence and incidence of CVS in the two groups.

Results: After the exclusion criteria, we evaluated 34 MS patients (median $\mathrm{FS}=2.5$, range 1-6) and $36 \mathrm{FD}$ patients (median $\mathrm{FS}=2$, range 1-4). While the presence of a positive CVS was detected at least in one FLAIR hyperintense lesion in 27/34 (79,4\%) MS patients, in the FD group none of the patients presented this sign. From these data, a negative predictive value of $83.7 \%$ was estimated.
Discussion: This study confirms that CVS is a neuroradiological sign characterized by a high incidence in MS patients, also in the early stages of the disease. On the other hand, the apparent absence of this sign in FD patients could be related to the different processes that lead to white matter damage in this condition compared to MS.

Conclusions: The CVS is a promising neuroradiological sign highly suggestive of MS, closely related to it pathophysiology and therefore it can be used to differentiate this condition from its possible mimickers.

\section{1-P41}

THE CLINICAL IMPACT OF BRAIN MICROSTRUCTURAL ABNORMALITIES IN MULTIPLE SCLEROSIS: A QUANTITATIVE MRI STUDY

MARIO TORTORA ${ }^{1}$, Mario Tranfa ${ }^{1}$, Giuseppe Pontillo ${ }^{1}$, Maria Petracca $^{2}$, Serena Monti ${ }^{3}$, Roberta Lanzillo ${ }^{2}$, Teresa Costabile ${ }^{2}$, Vincenzo Brescia Morra ${ }^{2}$, Sirio Cocozza ${ }^{1}$, Giuseppe Palma ${ }^{3}$, Arturo Brunetti ${ }^{1}$

${ }^{1}$ Department of Advanced Biomedical Sciences, University of Naples "Federico II", Naples, Italy, ${ }^{2}$ Department of Neurosciences and Reproductive and Odontostomatological Sciences, University of Naples "Federico II", Naples, Italy, ${ }^{3}$ Institute of Biostructure and Bioimaging, National Research Council, Naples, Italy

\section{Keywords: MRI, Neuroradiology, Multiple Sclerosis}

Introduction: The clinical impact of different brain microstructural abnormalities in multiple sclerosis (MS) remains poorly understood. In this light, quantitative MRI, assessing iron and myelin changes, represents a powerful tool to explore the clinical correlates of demyelination, neurodegeneration and tissue repair capacity. In this regard, we sought to characterize the topography of brain iron and myelin changes, together with atrophy, simultaneously assessing their impact on cognitive and motor disability in MS.

Methods: We conducted a prospective cross-sectional study of $117 \mathrm{MS}$ patients and 53 healthy controls (HC), performing voxel-based morphometry and voxel-based quantification analyses of longitudinal relaxation rate (R1, as a myelin marker) and quantitative susceptibility (QSM, as an iron marker) in gray matter (GM) and white matter (WM).

Differences between groups at the voxel level were evaluated with nonparametric permutation tests, while the correlations between MRI metrics and clinical variables (global disability, cognitive and motor performance) were assessed both globally and at the voxel level within clusters of significant between-group difference.

Results: We observed widespread reduction of R1 values in GM and WM regions, along with more limited changes in QSM and atrophy of deep GM, posterior and infratentorial regions, in MS patients compared to HC. While R1 and QSM were jointly reduced in several WM tracts, in GM the widespread R1 reduction was associated with decreased QSM in the thalamus and small clusters of increased susceptibility in the caudate nucleus and prefrontal cortex. Overall disability and motor performance were related, in addition to atrophy, to QSM values in the cingulate and corona radiata, while cognitive performance was related to the reduction of R1 in deep and periventricular WM.

Discussion \& Conclusion: Using quantitative MRI, we confirmed that diffuse demyelination occurs in the brains of MS patients, with more spatially limited changes of tissue QSM. Furthermore, in addition to the clinical impact of atrophy, we also demonstrated that motor and global 
disability are related to iron alterations, while cognition correlates with focal demyelination.

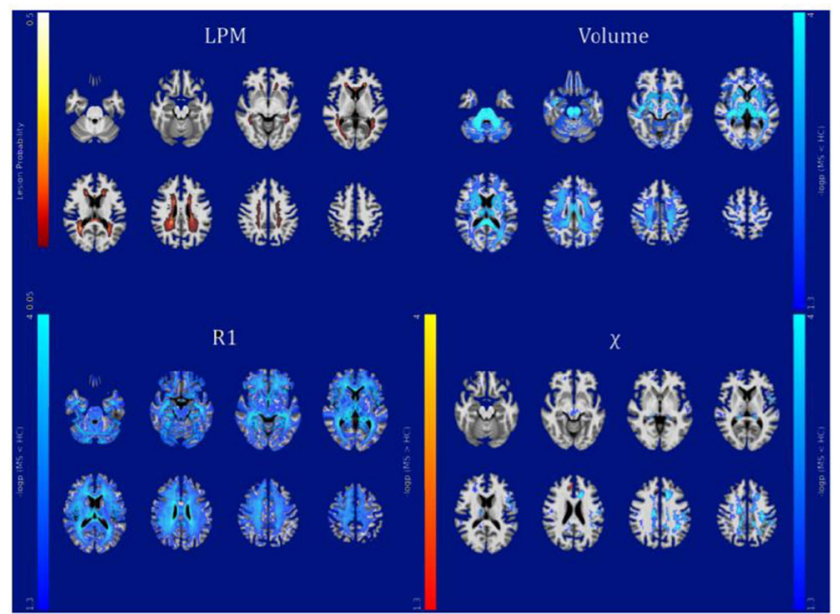

Figure 1 Results of the between-group voxel-wise comparisons.

A lesion probability map is presented (upper left panel), along with clusters of significant betweengroup difference in terms of volume (upper right panel), $\mathrm{R} I$ and $\mathrm{X}$ (lower panels) values for both the MS $>\mathrm{HC}$ (red-yellow) and MS< $<\mathrm{HC}$ (blue-light blue) contrasts.

\section{1-P42}

AMIDE PROTON TRANSFER (APT) IMAGING IN METASTASIS: PRELIMINARY RESULTS

$\underline{\text { LARS MICHELS }}^{* 1}, \underline{\text { BENCE NEMETH }}^{* 1}$, Valerie Treyer ${ }^{2,3}$, Andrea Bink $^{1}$

${ }^{1}$ Department of Neuroradiology, Clinical Neuroscience Center, University Hospital Zurich, University of Zurich, Zurich, Switzerland, ${ }^{2}$ Department of Nuclear Medicine, University Hospital Zurich, Zurich, Switzerland, ${ }^{3}$ Institute for Regenerative Medicine, University of Zurich, Schlieren, Switzerland

Keywords: metastases, brain, tumor, amide proton transfer

Introduction: Amide proton transfer (APT) imaging is a magnetic resonance imaging technique that detects endogenous mobile proteins and peptides in tissue via saturation of the amide protons in the peptide bonds. Previous studies have shown promise in detecting tumors and stroke. Recently, it was shown that APT imaging is a superior imaging technique to diffusion-weighted imaging (ADC) and 3-dimensional pseudo-continuous arterial spin imaging in the grading of gliomas [1,2]. However, it is not clear whether brain metastasis, manifested as secondary locations of non-brain tumors can be detected by APT. Hence, the relation between APT and standard tumor measures have not been yet assessed.

Methods: In 7 patients, APT imaging was performed on a 3 Tesla GE Scanner with the following parameters (slice thickness: $5 \mathrm{~mm}$, TR/TE: 10495/TE: $21.2 \mathrm{~ms}$, flip angle: $90^{\circ}$, FOV: 20x20 cm). DWI, FLAIR, T1, T1ce, T2 were acquired as well. Several tissue groups were defined and manually segmented in order to investigate the voxel intensities on different MRI sequences. Tumor class was further subdivided into glioma and intracranial metastases from lung cancer subclasses. Non-tumor class was divided into postresection reactive gliosis, other nonspecific white matter lesions, normal white matter and grey matter subclasses. The intermodal voxel-intensity differentiation was investigated between tumor and non-tumor groups and between tumor subclasses along the different MRI sequences. For that, Wasserstein metric, unpaired t-test, Signal-to-noise and contrast-to-noise ratios were calculated on the voxel intensities measured in the segmented and equally subsampled groups. In addition, Random Forest models were trained and optimized with weighted tumor classes to classify voxels and identify the importance of MR sequences.

Results: The most important sequences in terms of differentiation of tumor from non-tumor voxels based on t-tests and Wasserstein distances were the followings: APT-FreewaterT2/T1 $(\mathrm{t}=33.4, \mathrm{p}=1.159 \mathrm{e}-189)$, APT-M0c/M0a*T2a[s] $(\mathrm{t}=31.9, \mathrm{p}=1.110 \mathrm{e}-176), \mathrm{T} 2(\mathrm{t}=25.1, \mathrm{p}=1.965 \mathrm{e}-$ $119)$ and $\mathrm{T} 1 \mathrm{ce}(\mathrm{t}=24.6, \mathrm{p}=1.845 \mathrm{e}-115)$ sequences (Fig.1-2) Importance of images based on Random-Forest's Gini feature importance in descending order: APT-B0 (0.33), APT-Free waterT2/T1 (0.17), APT-Error index (0.07), T1-weighted contrast-enhanced (0.07) and FLAIR (0.05). SNR also highlighted the importance of FLAIR, APT-FreeWaterT2/T1, T1ce images (Fig3-4).

Discussion and Conclusion: Compared to other MR sequences, APTFreewaterT2/T1 images showed an improved role not only in differentiation of tumor and non-tumor voxels, but also in differentiation of glioma and metastases. In the future, we aim to test and confirm this in a larger sample of subjects and to predict the origin of metastatic tumors based on APT.
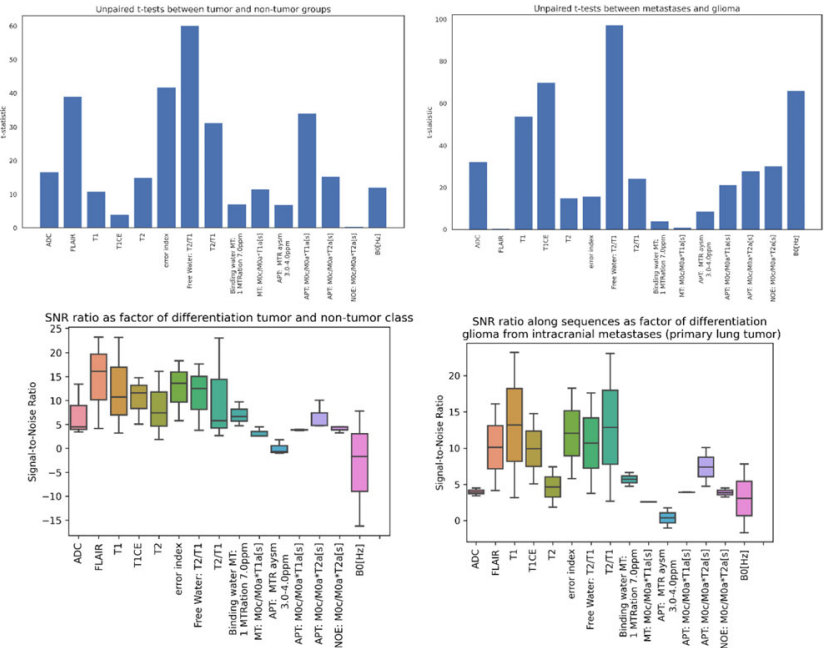

1-P43

ABILITY TO PREDICT LONG-TERM DISABILITY IN MULTIPLE SCLEROSIS USING A COMBINATION OF RADIOMICS AND MACHINE LEARNING

SIRIO COCOZZA ${ }^{1}$, Renato Cuocolo ${ }^{1}$, Giuseppe Pontillo ${ }^{1}$, Lorenzo Ugga $^{1}$, Maria Petracca ${ }^{2}$, Teresa Costabile ${ }^{2}$, Roberta Lanzillo ${ }^{2}$, Vincenzo Brescia Morra ${ }^{2}$, Mario Quarantelli ${ }^{3}$, Arturo Brunetti ${ }^{1}$

${ }^{1}$ Department of Advanced Biomedical Sciences, University of Naples "Federico II", Naples, Italy, ${ }^{2}$ Department of Neurosciences and Reproductive and Odontostomatological Sciences, University of Naples "Federico II", Naples, Italy, ${ }^{3}$ Institute of Biostructure and Bioimaging, National Research Council, Naples, Italy

Keywords: Neuroradiology, Multiple Sclerosis, RMI

Introduction: Based on MRI, volumetric and connectivity characteristics, we sought to detect the accuracy of Machine Learning (ML) models 
to predict cognitive risk (CI) and confirmed disability progression (CDP) in patients with multiple sclerosis (MS).

Methods: This retrospective evaluation was based on data obtained from 177 MS patients undergoing a 3T brain MRI and with a long-term (around 10 years) clinical follow-up available.

Motor function was assessed via EDSS at both baseline and follow-up, while cognitive functions were evaluated via BICAMS at follow-up. MRI protocol included a FLAIR sequence and a 3D T1- volume, Disconnection scores (inferred from the position and load of the white matter lesions defined on the FLAIR sequence) and radiomic characteristics from the atlas 116 regions of gray matter defined by the AAL atlas were extracted. Three different ML algorithms (Extra Trees, Logistic Regression and Support Vector Machine) were used to predict both CI and CDP, after selecting features at various stages in the training data $(n=132)$. Algorithm optimization was performed using 10-fold cross-validation in the training set with final model evaluation on the leak test set $(n=45)$.

Results: A total of 127764 features are extrapolated, of which 127549 are stable. After the removal of the low variance $(n=484)$ and highly interrelated $(=100860)$ parameters, the selection of the LASSO features identified a subset of 19 and 13 features, all but 2 from radiomics, for CI and CDP estimation, respectively. The model based on the recursive elimination of the features combined with the Extra Trees classifier employed 16 and 13 features to obtain the maximum precision for each endpoint, that was found to be 0.71 and 0.69 respectively.

Discussion \& Conclusion: Radiomics with an ML analysis promise to be a feasible and promising tool for long-term prediction of both cognitive and physical disability in MS patients.

\section{1-P44}

NOT TO FORGET: BILATERAL HIPPOCAMPAL LESIONS FOLLOWING ACUTE GLUFOSINATE AMMONIUM INTOXICATION

\section{RICARDO JOAO GASPAR PIRES ${ }^{1}$, Pedro Henrique Pestana Barradas $^{1}$, Daniela Jardim Pereira ${ }^{\mathrm{I}}$ \\ ${ }^{1}$ Functional Unit of Neuroradiology, Department of Medical Imaging, Coimbra Hospital and University Centre, Coimbra, Portugal}

Keywords: Herbicides, Glufosinate Ammonium, Poisoning, Hippocampus, Parahippocampal gyrus, Magnetic resonance imaging

Introduction: Glufosinate ammonium (GLA) is a commercial herbicide. Its use has increased exponentially over the last decade, as well as the incidence of intoxication through accidental or voluntary ingestion. Unlike other herbicides, patients typically present with neurological symptoms. An accurate diagnosis is critical for the management of the patient.

Methods: We report a case of an attempted suicide through ingestion of GLA, with characteristic brain MRI findings, followed by a brief literature review and discussion of main differential diagnosis.

Results: A 64-year-old male with no relevant previous medical history was admitted with intentional GLA $(50-100 \mathrm{~mL})$ intoxication. Initial assessment revealed type 1 respiratory failure $(\mathrm{pO} 2=54 \mathrm{mmHg}$ ) and acute confusional state, with no other remarkable findings. Later, a memory deficit for recent events was reported. A CT-scan, on the second week, showed new-found hypodense bilateral medial temporal lesions. Brain MRI, performed 1 month after intoxication, demonstrated symmetrical increased volume and signal on T2-weighted imaging of hippocampi and parahippocampal gyri, without restricted diffusion or contrast enhancement
A case-report search was conducted on PubMed and Google Scholar, using the terms "glufosinate ammonium" and "hippocampus". A total of 4 cases of anterograde amnesia with bilateral hippocampus involvement, following acute GLA intoxication, were found.

Discussion: The neurotoxicity by GLA may follow different pathways, with corresponding imaging changes, and neurologic symptoms may vary according to dosage. Isolated and nearly symmetrical hippocampal involvement on brain MRI, as in our case, is a macroscopic translation of the biochemical mechanisms mediating toxicity in the patient - since glufosinate is a glutamate analogous, it suggests excitotoxicity by overstimulation of glutamate-NMDA receptors. Autoimmune limbic encephalitis (ALE) was also considered - bilateral involvement of mesial temporal lobes is common $(60 \%)$, although usually asymmetric. In fact, a specific group of ALE operates through the same pathway, with antibodies acting against NMDA-receptors. HSV encephalitis may have similar (often asymmetric) findings, and possibly complicated by haemorrhagic lesions. Acute ischemic stroke in PCA territory can mimic a hippocampal lesion, though isolated hippocampal stroke, with no involvement of other structures, is rare, and should demonstrate restricted diffusion. Other metabolic encephalopathies can also affect the hippocampus.

Conclusion: Acute/subacute anterograde memory impairment, with symmetrical T2-weighted hyperintense hippocampal lesions, should raise suspicion for toxic/metabolic etiology. Although GLA intoxication is rare, it must be included in the differential diagnosis in the appropriate setting.

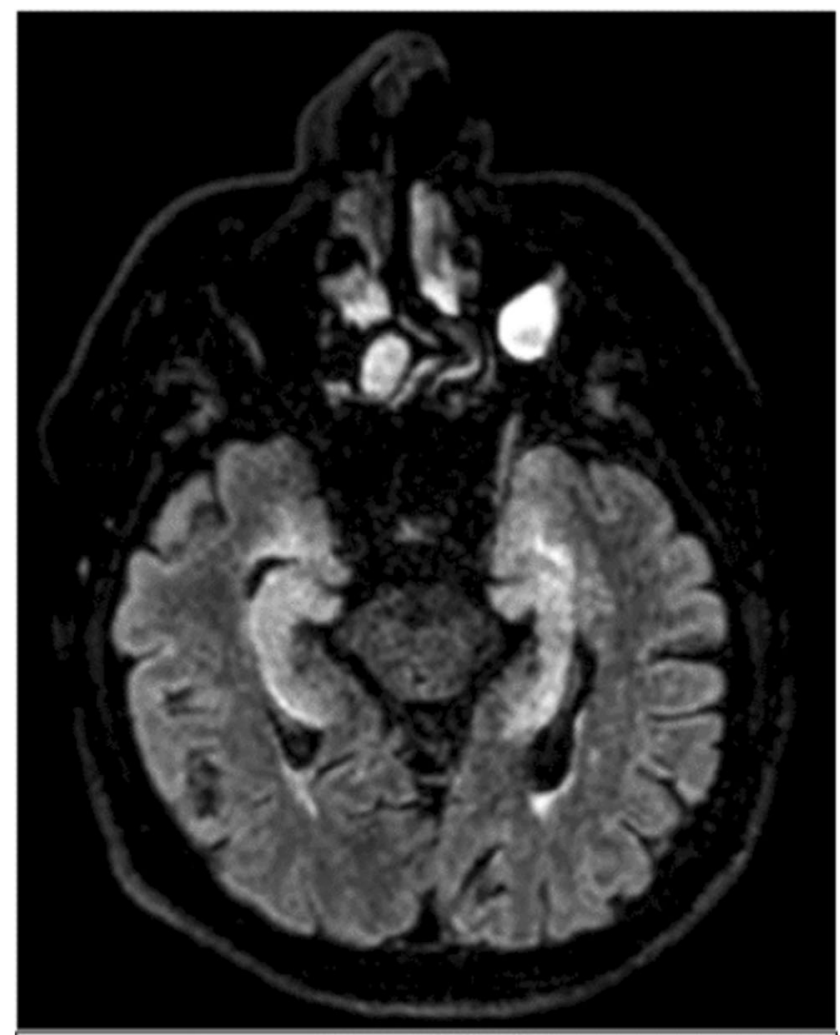

Brain MRI (1 month after presentation) - 3D-FLAIR with axial MPR, demonstrating nearly symmetrical hypersignal of hippocampi and parahippocampal gyrus, compatible with neurotoxicity by glufosinate ammonium. 


\section{1-P45}

HYPEREMESIS GRAVIDARUM COMPLICATED BY WERNICKE'S ENCEPHALOPATHY: CASE REPORT

\section{GIULIA CASTORANI $^{1}$, Filippo Savoldi ${ }^{2}$, Stefania Criscuolo ${ }^{2}$, Luca} Caschera $^{3}$

${ }^{1}$ Foggia University, Department of Radiology, Foggia, Italy, ${ }^{2}$ Universita statale di Milano, Department of Radiology, Milan, Italy, ${ }^{3}$ Fondazione IRCCS Ca' Granda Ospedale maggiore policlinico, Neuroradiology Unit, Milan, Italy

Keywords: Wernicke's Encephalopathy, Hyperemesis Gravidarum, Vitamin B1

Introduction: Wernicke's encephalopathy (WE) is a reversible neurological emergency induced by vitamin B1(thiamine) deficiency that, if untreated, can progress to coma and death.

Even if commonly associated with heavy alcohol consumption, it might also be seen in many other conditions: poor nutrition, malignancy, hyperemesis gravidarum( $\mathrm{HG})$ and prolonged intravenous feeding.

WE is recognized by the characteristic clinical triad: confusion, ataxia and ophthalmoplegia. The radiological evaluation contributes to clinical diagnosis and long-term maternal prognosis. We present a case of WE following HG in a healthy young woman, where clinical findings were supported by magnetic resonance imaging (MRI) of brain.

Methods \& Results: A 27-year-old pregnant woman at 17 weeks was admitted in our hospital with a history of weight loss, right upperquadrant pain, nausea and vomiting. She had one previous successful pregnancy and a history of hyperthyroidism on Propylthiouracil-treatment. Alcohol use was denied, and urine drug screen was negative.

Laboratory tests showed elevated serum liver transaminase levels. She had been treated with intravenous fluids for HG without much improvement during the previous 1 month.

The patient later developed neurologic symptoms including ataxia, confusion, short-term memory loss and, long after, nystagmus, dysphonia and dysphagia. Therefore, the patient was transferred to ICU. The suspicion for WE was confirmed by characteristic findings on brain MRI. It revealed symmetrical signal abnormalities in T2/FLAIR images involving mainly the mammillary bodies, the walls of hypothalamus and the postero-medial thalami, with only minimal diffusion restriction. Intravenous thiamine was started with a regimen of $500 \mathrm{mg}$ three times per day for 2 days and later $250 \mathrm{mg}$ per day for 5 days, followed by $100 \mathrm{mg}$ oral tablet daily. Over the next two weeks, there was gradual improvement in encephalopathy with only residual weakness of the lower limbs.

Discussion \& Conclusion: Vitamin B1 is an essential water-soluble vitamin easily assumed by adult diet. However, in pregnancy the requirement of thiamine may increase, especially if complicated by HG. Brain MRI is extensively used for the initial diagnosis of WE and to rule out other differential conditions. The typical hyperintensities on FLAIR and T2-wi involve the most sensitive areas to thiamine deficiency due to cellular dependence on oxidative metabolism (medial aspect of thalami, mammillary bodies, periaqueductal regions and mesencephalic tegmentum). In conclusion, WE is a life threatening condition with potential neurologic sequelae in the mother and complications for the fetus, which can easily resolved with a prompt diagnosis and parenteral thiamine.

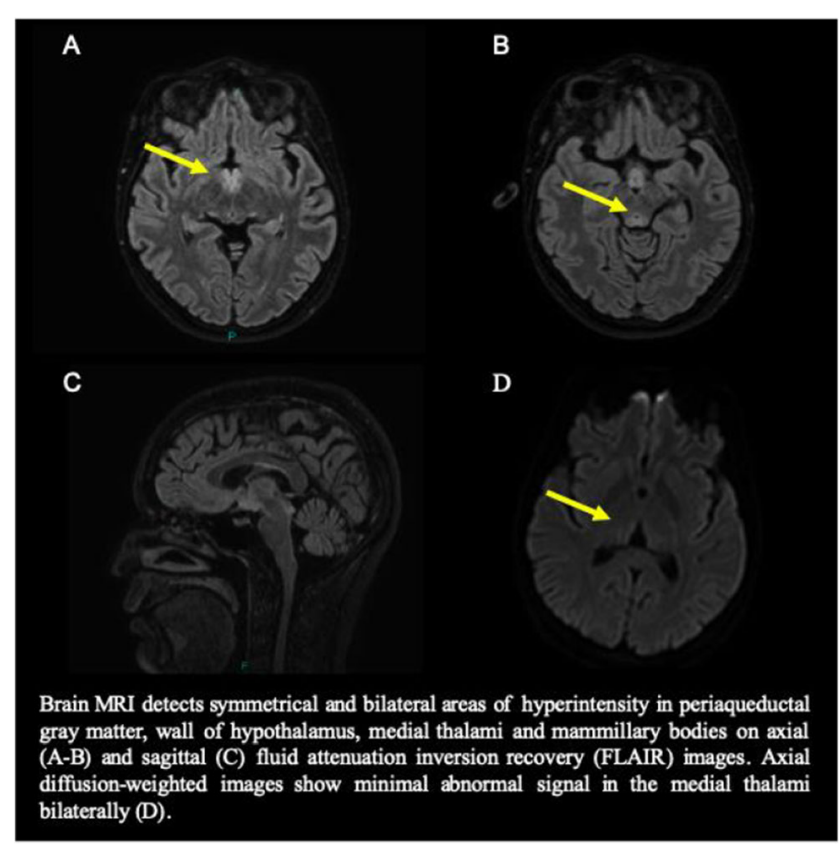

1-P46

HEREDITARY SPASTIC PARAPLEGIAS: PICTORIAL REVIEW AND NOVEL MR IMAGING FINDINGS

\section{CAROLINA MAIA $^{1}$, Ines Cunha ${ }^{2}$, Joana Ribeiro ${ }^{2}$, Rui Pais ${ }^{1}$, Joana Pinto $^{1}$}

${ }^{1}$ Medical Image Department, Neuroradiology Unit, Coimbra University Hospital Center, Coimbra, Portugal, ${ }^{2}$ Neurology Department, Coimbra University Hospital Center, Coimbra, Portugal

Keywords: hereditary spastic paraplegia, SPG, magnetic resonance imaging, neurodegeneration

Introduction: Hereditary spastic paraplegias (HSP) are a large group of neurodegenerative diseases, genetically and clinically heterogeneous. MR imaging emerges as an important tool to assist in the exclusion of HSP-mimics, to guide genetic testing and to understand the pathophysiology of the disease. Despite that, there are few studies devoted to characterization of neuroimaging abnormalities in HSP.

Methods: We report four rare cases of HSP with genetic diagnosis from our institution database, with novel MR imaging findings. Additionally, we provide a review of the different neuroimaging features and their related genetic HSP subtypes.

Results: A 45-years-old man presented with early-onset slowly progressive spastic paraparesis. Brain-MRI showed bilateral T2 and SWI hypointensity in the globus pallidus, substantia nigra, red nucleus and dentate nucleus, suggestive of iron accumulation. Genetic investigation was compatible with autosomal dominant spastic paraplegia (SPG) type 31.

A 50-years-old women, born from a consanguineous couple, began a leftsided paresis in childhood, which slowly evolved to spastic paraplegia, sphincter dysfunction and mild cerebellar ataxia. The FLAIR image shows cone-shaped high signal intensity extending from the anterior horns of the lateral ventricles, with corresponding low signal intensity on the T1-weighted image, called the "ears-of-the-lynx" sign. Although 
this sign point to SPG11 or SPG15, genetic investigation disclosed an autosomal recessive SPG5 gene mutation.

The last cases are of two non-related 55-years-old male and female, with a pure progressive spastic paraparesis and genetic diagnosis of SPG4. Both performed a Brain-MRI that showed the "hummingbird" sign reflecting mesencephalic atrophy.

Discussion \& Conclusion: To the best of our knowledge, we present novel neuroimaging findings in SPG31, SPG5 and SPG4, not yet described in the literature. Although nonspecific, they could give an additional clue to a genetic HSP diagnosis.

Considering the rarity of HSP subtypes, multicentric studies should be designed to obtain representative cohorts of patients and fully appreciate how neuroimaging can help us to diagnose and understand the pathophysiology underpinning these complex diseases.

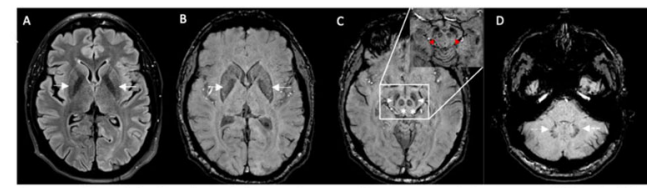

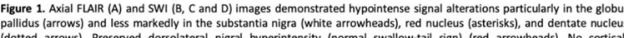

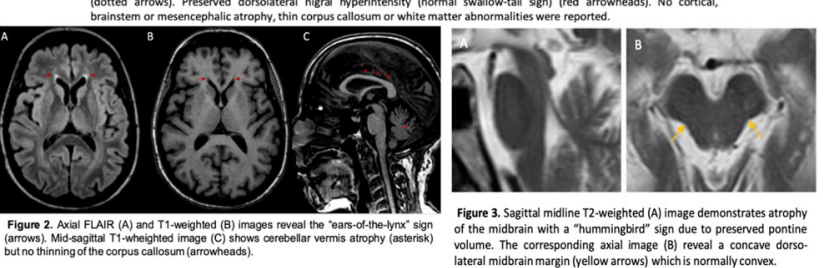

1-P47

NEUROIMAGING IN SICKLE CELL ANEMIA

LAISE RAMOS NERI ${ }^{1}$, Livia Porto Teixeira ${ }^{1}$, Katia Nakacima Terumi ${ }^{1}$, Luziany Araujo ${ }^{1}$, Lais Fajardo Ramin ${ }^{1}$, Lisa Suzuki ${ }^{1}$, Suely Fazio Ferraciolli $^{1}$, Claudia da Costa Leite ${ }^{1}$, Leandro Tavares Lucato ${ }^{1}$

${ }^{1}$ University of Sao Paulo, Sao Paulo, Brazil

Keywords: sickle cell anemia, neuroimaging, cerebrovascular complications

Introduction: Sickle cell disease (SCD) is the most common genetic hemoglobinopathy and is associated with serious complications that can affect the brain parenchyma, vasculature, and skull.

Methods: We selected didactic and representative neuroimaging features of SCD from cases of our institution.

Discussion: Neuroradiological findings in vasculature complications include acute territorial infarction, silent ischemia, and intracranial hemorrhage. Intracranial large artery infarction is the most commonly recognized stroke syndrome in children with SCD. Sickle cell vasculopathy typically involves the terminal internal carotid artery, proximal anterior and middle cerebral artery, leading to stenosis. With progressive occlusion of the main intracranial arteries, a so-called "Moya Moya" (Japanese: a puff of smoke) appearance is seen and represents the development of collateral vessels. Steno-occlusive change can occur not only in the internal carotid artery but also in the posterior cerebral artery. Silent ischaemia typically occurs at the "watershed" border zones of vascular territories and they are usually relatively small brain lesions. Patients are at risk of hemorrhage within the parenchymal, which may be caused by friable and dilated collateral vessels or hemorrhagic transformation of the ischaemic brain; subarachnoid hemorrhage, due to the ruptured aneurysm, the most common intracranial hemorrhage cause; and hemorrhage within the subdural/extradural compartments, that may complicate bone infarction. Volumetric MRI studies of patients with SCD have shown parenchymal volume loss. Posterior reversible encephalopathy syndrome has been reported in association with acute chest crisis, steroid use, over transfusion, and sepsis. Cerebral fat embolism secondary to the breakdown of infarcted bone is a rare complication of SCD. Calvarial bone infarction is another uncommon complication, with imaging demonstrating bone infarction, spontaneous extradural hemorrhage, or subperiosteal hemorrhage.

Conclusion: It is important for the neuroradiologist to know possible brain imaging findings associated with SCD since they have an important role in the screening, diagnosis, and management of these patients.
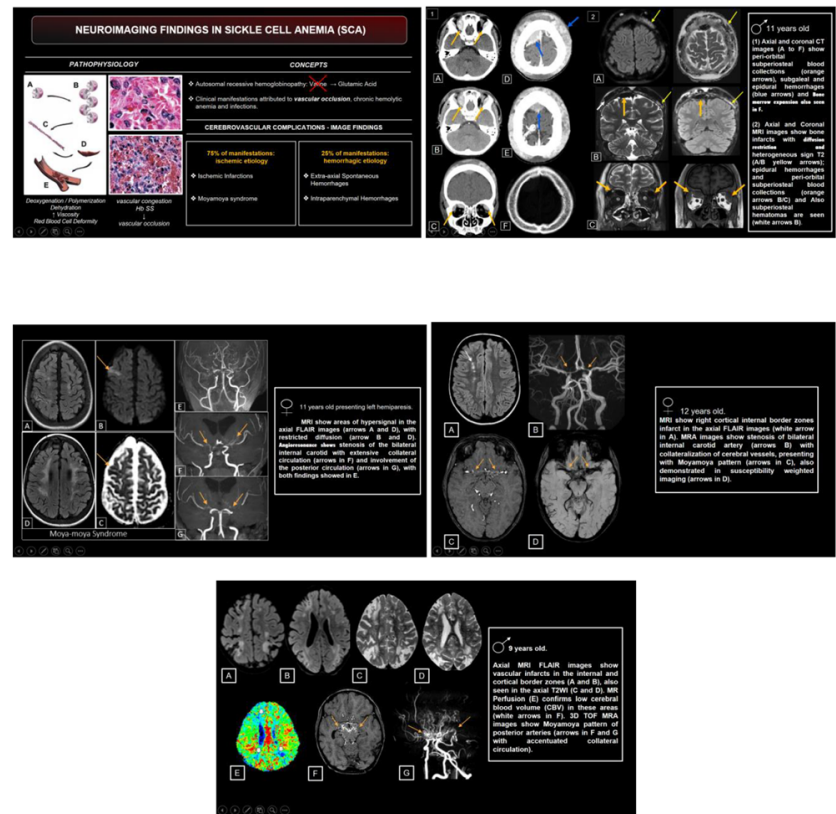

1-P48

INCIDENTAL INTRACRANIAL CT FINDINGS THAT ARE OFTEN MISCALLED

$\underline{\text { BERNA AYGUN }}{ }^{1}$, Calvin Soh $^{2}$

${ }^{1}$ Department of Radiology, Manchester University NHS Foundation Trust, Manchester, UK, 2 Department of Neuroradiology, Manchester University NHS Foundation Trust, Manchester, UK

Keywords: Computed tomography, Brain, Incidental findings

Computed tomography (CT) brain is a vastly available, quick and sensitive screening tool in the emergency setting. Between 2019 and 2020 there were over 5 million CT scans performed on the NHS patients. These are often evaluated by non-specialist radiologists or clinicians during oncall hours which increases the risk of miscalling incidental findings. Incidental findings are unrelated to the main presenting complaint and do not warrant urgent attention in emergency setting. In the literature, a number of retrospective and prospective are available identifying these findings both evaluating control groups in healthy volunteers and in emergency setting. However, there is a variation in prevalence due difference in population age and cohort demographics. Running these 
studies are also ethically challenging because of potential impact of the incidental findings on the patient's care and mental health and there are no clear guidelines to follow.

In this educational review we summarize the commonest CT incidental findings that do not require further follow-up and are not pertinent to patient care. The commonest lytic pseudo lesions of the calvarium with filling defect of the diploic space include arachnoid granulations and venous lacunae. Appreciating the benign features with well-defined contours, location and involvement of the inner table would easily help to differentiate these lesions even in challenging circumstances such as in the case of giant arachnoid granulations. Intraosseous haemangiomas are mostly asymptomatic and appears expansive lesion with scalloping of the outer table characterised with spicules that are radiating from a common centre. Intracranial calcifications which are physiological occur in common locations (basal ganglia, hibernaculum, pineal gland) with increasing incidence with patient's age. Choroidal plexus calcification in the unusual places such as temporal horns or foramina Luschka should not be confused with subarachnoid haemorrhage in the acute setting. Similarly familiarising with imaging characteristics of xanthogranulomatous degeneration of choroid plexus would prevent overcalling cystic heterogenous lesions of the choroid plexus. These rarely cause hydrocephalus and very rarely require intervention. Arachnoid cysts, in particularly in the posterior fossa, should be distinguished from other differentials by its typical features of bone scalloping creating a convexity. Enlarged perivascular spaces adjacent to anterior commissure maybe mistaken for lacunar infarcts, similarly in the temporal pole maybe mistaken for cystic tumours. Magnetic resonance imaging (MRI) brain in these rare cases may be used in conjunction to help differentiating usual incidental findings in unusual locations and atypical presentation.

\section{1-P49 \\ INTERNAL CAROTID ARTERY DISSECTION RELATED TO ABNORMALITIES OF STYLOID PROCESS: A RETROSPECTIVE SINGLE CENTER CASE-CONTROL STUDY}

NICOLA ROMANO ${ }^{1}$, Valentina Tardivo ${ }^{2}$, Margherita Federici ${ }^{1}$, Aldo Fischetti ${ }^{1}$, Simona Parodi ${ }^{1}$, Uberto Luigi Ruffinengo ${ }^{1}$, Giacomo Siri ${ }^{3}$, Antonio Castaldi ${ }^{1}$

${ }^{1}$ Department of Diagnostic and Interventional Neuroradiology, Galliera Hospital, Genoa, Italy, ${ }^{2}$ Neurosurgery Unit, Galliera Hospital, Genoa, Italy, ${ }^{3}$ UCS Scientific Directorate, Galliera Hospital, Genoa, Italy

Keywords: carotid artery, CT, dissection

Introduction: Vascular Eagle syndrome caused by impingement of the extracranial internal carotid artery (ICA) by the styloid process (SP) is an uncommon and not yet widely recognized cause of ICA dissection. Up to now, this diagnosis is still presumptive, based mainly on the length of the SP. However, other anatomical factors beyond the length itself of this bony structure seem to be involved.

Material \& Methods: We reviewed Computed Tomography Angiography (CTA) examinations of ICA dissection from 2009 to 2019 in our Institution, performing a retrospective single center casecontrol study of ICA dissection related to abnormalities of SP and ageand sex-matched controls affected by ICA dissection not related to abnormal relationship with the SP. In our work instead of considering SP length as the main criteria to differentiate the two groups, we decided to consider SP-ICA distance (at the dissection point) as the main factor to define a styloid process related dissection (SPRD). In fact in some patients, the distance between the dissected artery and the bony prominence was virtual.
Results: 41 patients, of which $10(24.39 \%)$ had SPRD, were included in the study. In patients with SPRD the styloid process angulation on the CT coronal plane tended to be more acute and the styloid process- $\mathrm{C} 1$ distance was significantly shorter at the side of the dissection.

Discussion \& Conclusion: Our results reinforce the idea that ICA dissection risk in the vascular Eagle syndrome has probably a multifactorial pathogenesis: the anatomic relationships between SP, ICA, and C1 lateral process should be always taken into account in patients with ICA dissection.

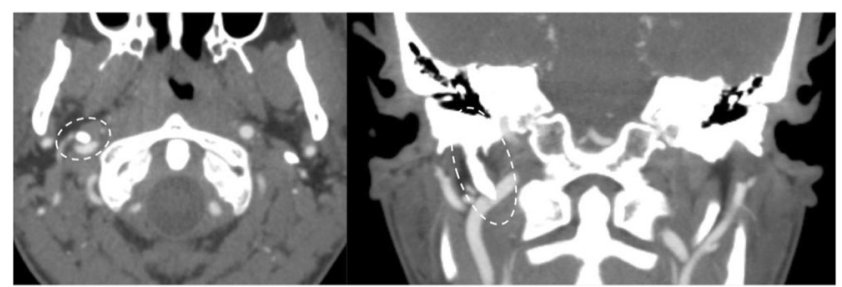

\section{1-P50}

HYPERINTENSE ACUTE REPERFUSION MARKER (HARM) - A CONFUSING SIGN IN ACUTE STROKE IMAGING

\section{NORA LUCA NYILAS ${ }^{1}$, Pal Maurovich-Horvat ${ }^{1}$, Peter Barsi $^{1}$}

${ }^{1}$ Department of Neuroradiology, Medical Imaging Centre, Semmelweis University, Budapest, Hungary

Keywords: Ischemic Stroke, Magnetic Resonance Imaging, Cerebrospinal Fluid, Reperfusion Injury

Introduction: HARM is an early and transient sign of blood brain barrier (BBB) disruption caused by leakage of gadolinium-based contrast material (GBC) into the cerebrospinal fluid (CSF). HARM was initially described in acute ischemic stroke and is seen as a hyperintense signal within CSF on postcontrast fluid-attenuated inversion recovery (FLAIR) image. A proposed mechanism for BBB disruption is the activation of inflammatory cascades and proteolytic enzymes after sudden hemodynamic instability. Correlation with clinical outcome is controversial - according to the latest studies the prognosis is favorable in cases of HARM with no or only small infarction.

Methods: From our MRI database we have retrospectively studied patients with acute stroke symptoms who had an initial MRI examination and underwent follow-up brain MRI within 48 hours of hospitalization from February 2020 to June 2021. Follow-up axial FLAIR image parameters: $5 \mathrm{~mm}$ slice thickness, no gap, 16ms ETL, 240x180x150 mm FOV, TR/TE/TI of $11,000 / 100 / 2800 \mathrm{~ms}$, flip angle of 90 and 192x134 matrix. HARM was defined as focal hyperintensity in the subarachnoid space and/or ventricles on FLAIR images.

Results: In total, we have included 20 patients ( 7 men and 13 women; mean age $70 \pm 9$ year) in our study. Initial MRI examination was performed with double dose GBC (Contrast-enhanced MR angiography, Dynamic susceptibility contrast MR perfusion). The median elapsed time till follow-up imaging was 26 hours (IQR=3.5 - 45h). On the follow-up MRI 10 patients showed HARM, 9 with a restricted diffusion (DWI) lesion (median core volume $=1 \mathrm{~cm}^{3}, \mathrm{IQR}=0.3-8 \mathrm{~cm}^{3}$ ) and 1 without DWI lesion (diagnosed as repetitive TIA). We found no association between HARM and age $(\mathrm{p}=0.2)$, last seen well $(\mathrm{p}=1.0)$, elapsed time till follow-up MRI ( $\mathrm{p}=0.36$ ). There was a tendency, but no correlation between HARM and anterior circulation disturbances $(\mathrm{p}=0.3)$, and small 
cortical lesions ( $\mathrm{p}=0.58$ ). 11 patients had a 90-day follow-up MRI: 6 of them had HARM previously and all of them completely resolved.

Discussion \& Conclusion: HARM was a frequent finding in acute stroke patients who underwent short interval follow-up MRI after an initial, contrast enhanced MRI. In our database a tendency, but no correlation was found between HARM and anterior circulation disturbances and small cortical lesions. All of them resolved on long term follow-up. Limitations of this study are the retrospective design, relatively small sample size and the lack of cases with revascularization therapy. Nevertheless, neuroradiologists should be aware of the phenomenon to avoid inappropriate patient management.

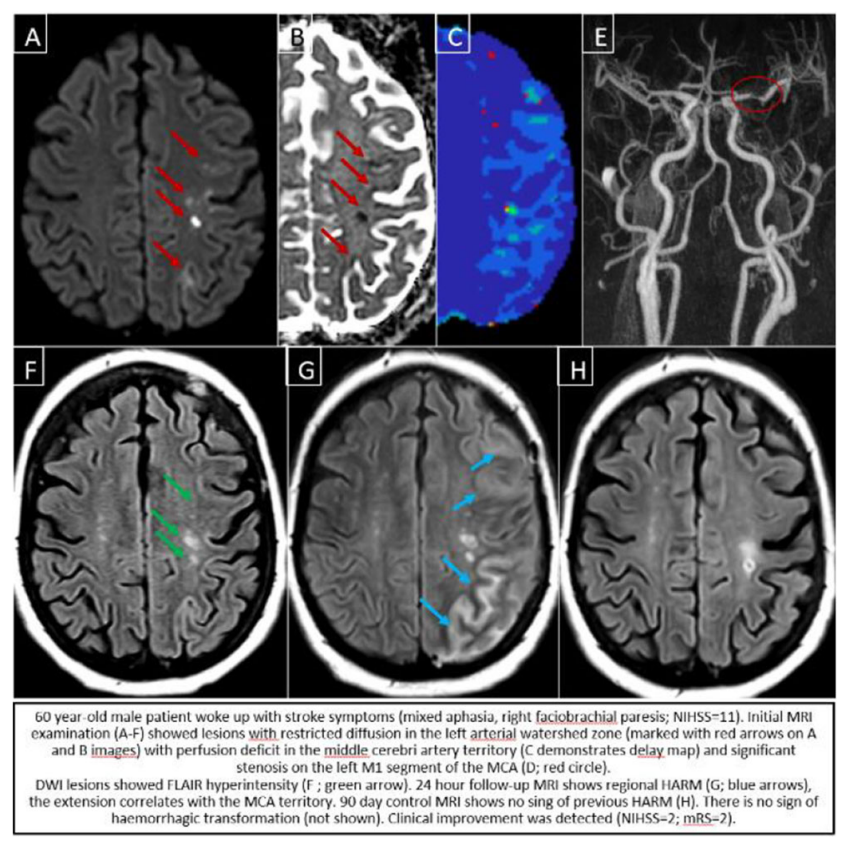

\section{1-P51}

A BOW HUNTER'S SYNDROME CASE REPORT - THE IMPORTANCE OF USING DYNAMIC NEUROIMAGING TECHNIQUES

JOAO TARRIO $^{1,2}$, Catarina Mendes Pinto ${ }^{1}$, Eduarda Pinto ${ }^{1}$, Joao Pedro Filipe $^{1}$, Cristina Ramos ${ }^{1}$, Viriato Alves Alves ${ }^{1}$

${ }^{1}$ Centro Hospitalar Universitario do Porto, Department of Neuroradiology, Porto, Portugal, ${ }^{2}$ Hospital Central do Funchal, Department of Neuroradiology, Funchal, Portugal

Keywords: bow hunter's syndrome, positional vertebral artery insufficiency, dynamic digital subtraction angiography

Introduction: Bow hunter's syndrome (BHS) is a symptomatic vertebrobasilar insufficiency resulting from a rotational stenosis or occlusion of a vertebral artery (VA). It is rare and motivated by dominant VA dynamic compression by abnormal bony structures, such as osteophyte, disc herniation, cervical spondylosis, tendinous bands or tumors. BHS symptoms range from transient vertigo and visual blur to posterior circulation stroke. Diagnosis is based on dynamic computed tomography angiography (CTA) and confirmed with a provocative dynamic digital subtraction angiography (DSA). The treatment can be conservative, surgical or endovascular, choice depending on several factors including the etiology, severity, occupation and lifestyle. The meaning of "bow hunter" is that in addition to a posture that requires right-angle head rotation, there is commonly also stabilization of an arrow fixed with the hunter's thumb in its occiput due to the high force used.

Methods: Clinical and radiological case description and discussion.

Results: A 70-year-old male, autonomous, ex-smoker, with type 2 diabetes mellitus, dyslipidemia and bilateral peripheral arteriopathy with revascularization of the left lower limb, complained, during a routine consultation, of recurring bilateral amaurosis fugax crises with right cervical rotation. He was sequentially studied by carotid doppler ultrasound (CDU), CTA and DSA. The CDU was described as normal. A CTA was performed in neutral position and with right cervical rotation, demonstrating an indentation of the right $\mathrm{VA}$ in the $\mathrm{C} 3-\mathrm{C} 4$ plane, caused by a right lateral disc protrusion and osteophyte, resulting in a focal caliber reduction of about $50 \%$ in the right cervical rotation. There was also diffuse atheromatous disease of the supra-aortic trunks. In the DSA, contrast injections in the right VA in neutral position and with cervical rotation maneuvers, revealed focal caliber reduction of approximately $50 \%$ in the distal V2 segment, upon rotation to the right. After diagnosis and weighing the risk-benefit, a conservative approach was chosen. The patient avoided head rotation movements and had no further symptoms.

Discussion \& Conclusion: Symptoms of vertebrobasilar insufficiency after head rotation in adults, especially males with $50-70$ years, should be suspected of BHS. Neuroimaging examinations can demonstrate abnormal surrounding structures, ischemic lesions and stenotic arteries. The final diagnosis should be made with DSA, showing the patent arteries in the neutral position and the stenotic arteries in the rotated position.
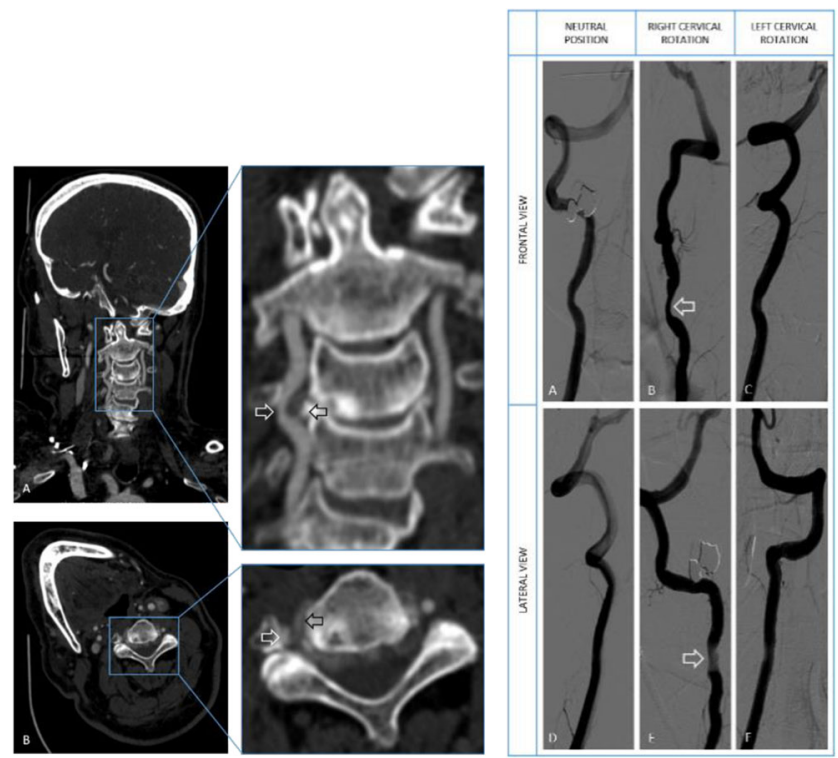

\section{1-P52}

POSTERIOR FOSSA TUMORS: AN UPDATE IN THE ERA OF GENOMICS

KAROLINA PINTO PELEGRINI CANCELA ${ }^{1}$, Ana Paula Alves Fonseca $^{1}$, Erica Antunes Naves ${ }^{1}$, Aline Almeida Baptista de Oliveira Salgado $^{1}$, Rita Maciel Pincerato ${ }^{1}$

${ }^{1}$ Department of radiology and diagnostic imaging, Hospital Samaritano, Sao Paulo, Brazil 
Keywords: posterior, fossa, tumors

Introduction: Posterior fossa tumors represent a significant percentage of CNS tumors, especially in the pediatric group. Among the main subtypes, the most important are medulloblastoma, pilocytic astrocytoma, ependymoma, hemangioblastoma and metastasis. The objectives of this exhibition are to review the anatomy of the posterior fossa, including its main anatomical boundaries and the structures that compose it, including the cerebellum, brainstem, meninges and cranial nerves. Also, discuss and illustrate the imaging patterns of major posterior fossa tumors.

Methods: After reviewing our institution's digital teaching files, we selected some illustrative cases to address diagnostic scenarios of some posterior fossa tumors, focusing on typical imaging patterns, unexpected findings, tumor spread patterns, and post-treatment imaging aspects. In addition, correlate with current classification, histological and molecular aspects, when available.

Results: In our series, we describe some subtypes of posterior fossa tumors. Among them, we highlight cerebellar tumors, which were represented by medulloblastoma, pilocytic astrocytoma, teratoma, atypical rhabdoid teratoid tumor, ependymoma, hemangioblastoma, metastasis and chloroma. Brainstem tumors, in turn, were characterized by diffuse glioma and rosette-forming glioneural tumor. From extra-axial tumors, we portray meningioma, schwannoma and hemangiopericytoma.

Discussion: A considerable part of CNS tumors are from the posterior fossa. MRI can be used in the assessment of these conditions, including techniques that provide information about the chemical composition of these tumors to help the radiologist make a specific diagnosis. Among the most common subtypes, pilocytic astrocytoma can have the classic appearance of a large cyst with a solid mural nodule within one of the cerebellar hemispheres; classic medulloblastoma, in turn, is a highly cellular and compacted tumor, presenting hyperdense on $\mathrm{CT}$, with restricted diffusion in the DWI, in addition to low ADC. Ependymoma, on the other hand, usually originates in the floor of the IV ventricle, with calcification in some cases and heterogeneous contrast enhancement. Given these and other characteristics, it is important to review the radiological characteristics of posterior fossa tumors.

Conclusion: The radiologist must be familiar with the different imaging presentations of posterior fossa lesions, with the objective of an accurate and early diagnosis, enabling an adequate treatment, often avoiding lesions in substantial brain structures, always aiming at a better prognosis for these patients, in addition to evaluating recurrences.

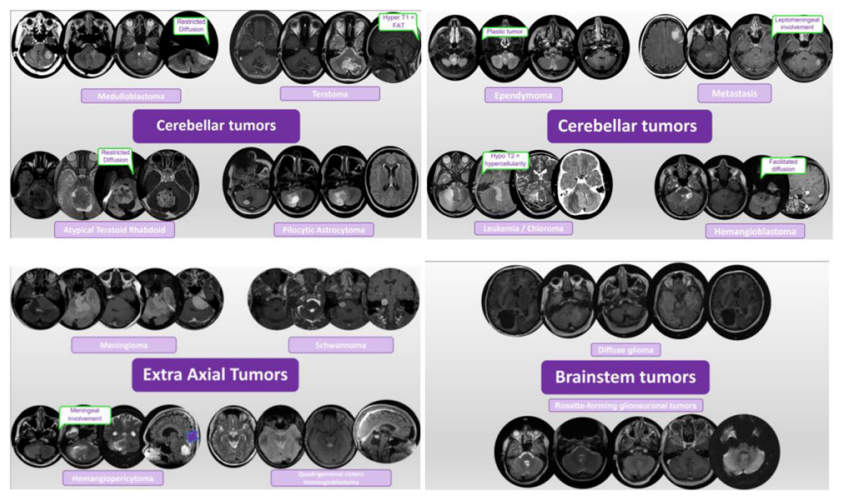

1-P53

AN ANALYSIS OF BRAINOMIX E-ASEPCTS SOFTWARE

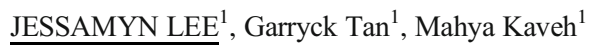

${ }^{1}$ Department of Radiology, Darent Valley Hospital, Dartford, United Kingdom

Keywords: Stroke, e-ASPECTS, CT Head

Background \& Aims: The Brainomix e-ASPECTS software is an AI tool used to assess for hypodensity on CT head images, giving a score that reflects the Alberta Stroke Program Early CT Score (ASPECTS) for middle cerebral artery (MCA) strokes. Furthermore, it was shown in previous studies to be able to report at an expert level. This study aims to compare e-ASPECTS output and neuroradiologist reports to evaluate whether the e-ASPECTS can be used as a reliable component in local stroke pathways to inform management.

Methods: A retrospective analysis was performed on CT head images of 55 consecutive confirmed acute stroke and 55 non-stroke patients in a district general hospital. The Categorical Model was used to anaylse and assess the congruency of the e-ASPECTS scores in relation to the consultant neuroradiologist; relevant reports were then used to establish factors affecting the outcome generated.

Results: There was an agreement between findings of e-ASPECTS software and consultant neuroradiologist, where the Precision and Recall percentages were $60 \%$ and $89.4 \%$ respectively, resulting in an F1 score of $71.8 \%$. The e-ASPECTS values were affected by imaging artefacts, bleeds and shifts in position of brain areas. However, it was unable to evaluate areas of extended MCA and watershed territories.

Conclusions: The Brainomix e-ASPECTS software can potentially be used to aid stroke diagnosis and help guide management in hospital stroke pathways. Nonetheless, there are limitations that need to be considered.

\section{1-P54}

BE CAREFUL AND STAY SAFE! CNS INVOLVEMENT BY COVID-19 CASE-BASED

ANA PAULA ALVES FONSECA ${ }^{1}$, Karolina Pelegrini Cancela ${ }^{1}, \mathrm{~T}$. Iutaka $^{1}$, Marcelo Guedes ${ }^{1}$, Erica Antunes Naves ${ }^{1}$, Aline Almeida Baptista de Oliveira Salgado ${ }^{1}$, Rita Cassia Maciel Pincerato ${ }^{1}$

${ }^{1}$ United Health Group, Neuroradiology Division, Sao Paulo, Brazil

Keywords: COVID-19, MRI, Brain

Purpose: A large spectrum of neurologic disease has been reported in patients with coronavirus disease 2019 (COVID-19) infection. Our aim was to Review the involvement of the CNS by COVID-19, detailing the pathophysiology through direct and indirect pathways, and to discuss and illustrate the main clinical and imaging scenarios of CNS involvement by COVID-19, from vascular, inflammatory, infectious and post-infectious disorders.

Methods/Approach: After reviewing our institution teaching files, we selected cases demonstrating clinical and imaging scenarios of CNS involvement by COVID-19, including: vascular (ischemia, hemorrhage, PRES, anoxia, cerebral venous thrombosis); inflammatory and infectious involvement (encephalitis, meningitis, neuritis, leukoencephalopathy); postinfectious involvement (acute necrotizing encephalopathy and 
Guillain-Barre); spinal cord involvement (myelitis; spinal cord infarction); and metabolic complications (acute uremia; cytotoxic lesions of the corpus callosum; osmotic demyelination).

Conclusion: The pathobiology of these neuroinvasive viruses is still incompletely known, and it is therefore important to explore the impact of COVID-19 infections on the nervous system. Here, we review the research into neurological complications in $\mathrm{CoV}$ infections and the possible mechanisms of damage to the nervous system.
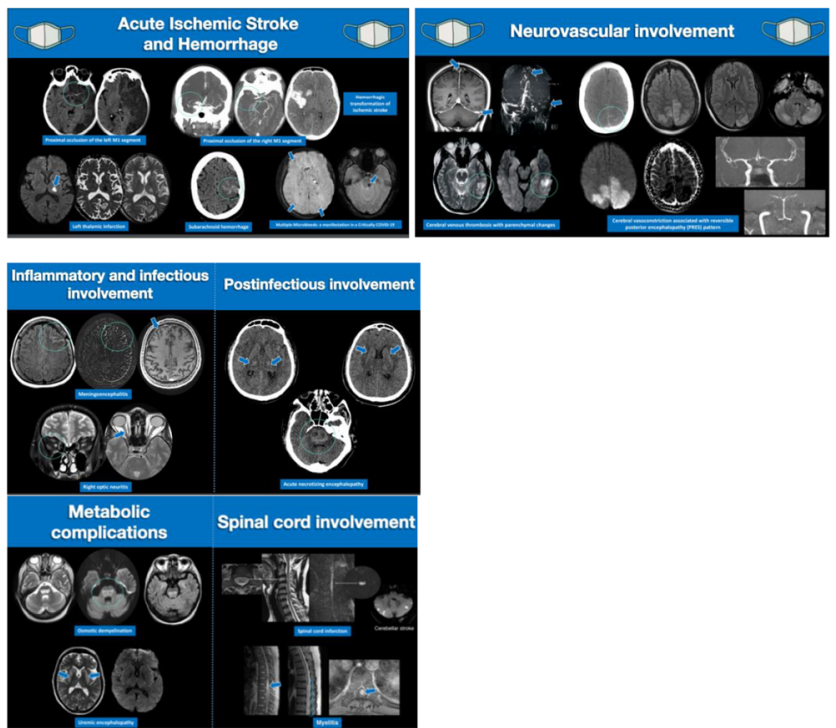

\section{1-P55 \\ MRI FINDINGS IN PRIMARY BRAIN LYMPHOMA. A PATTERN- BASED APPROACH}

ANDJOLI DAVIDHI ${ }^{1}$, Fernando Mas Estelles ${ }^{2}$, Regina Teruel-Coll ${ }^{2}$, Miguel Mazon-Monparler ${ }^{2}$, Fernando Aparici Robles ${ }^{2}$, Joan CarreresPolo ${ }^{2}$

${ }^{1}$ Department of Radiology, University General Hospital of Thessaloniki A.H.E.P.A., Stilp. Kiriakidi 1, 54636, Thessaloniki, Greece, ${ }^{2}$ Servicio de Radiologia, Area Clinica de Imagen Medica, Hospital Universitario y Politecnico La Fe, Avinguda de Fernando Abril Martorell, 106, Valencia 46026, Spain

Keywords: primary brain lymphoma, MRI imaging patterns, DDx

Introduction: Primary brain lymphoma (PBL) is an uncommon neoplasm with poor prognosis. It represents less than $4 \%$ of CNS tumors and it is characterised by no evidence of systemic involvement. Due to PBL's varying imaging patterns, the differential diagnosis is difficult (neoplasms, infections, demyelinating and vascular disorders). Nonetheless, the imaging patterns of conventional MRI combined with advanced MRI techniques can be used to, accurately, establish a differentiation of PBLs leading to an early diagnosis and guiding neurosurgeons to choose the most appropriate preoperative plan.

Methods: A retrospective search was carried out in the database of the neuroradiology section of our hospital. All MR studies performed on 1.5 and $3 \mathrm{~T}$ equipments of histologically confirmed PBLs were reviewed. They were classified according to the imaging patterns of conventional MR sequences. When available, their characteristics in advanced sequences were gathered (diffusion, perfusion and spectroscopy).
Results: PBLs demonstrated a variety of MR imaging patterns in our patients. Except for its typical MR features (periventricular, homogeneous, hypointense on T1/T2 lesion), PBL showed atypical imaging patterns such as perivascular enhancement or isolated ependymal contrast enhancement with or without intraparenchymal mass, "butterfly" pattern, and isolated meningeal enhancement. Moreover, the immunosuppressed patients (e.g. HIV-related) presented either with multiple ring-enhancing tumors with necrotic components or with necrotic and haemorrhagic masses. Other patterns were that of diffuse-infiltrative white matter lesions (e.g. lymphomatosis cerebri) or infarct-like lesions (e.g. intravascular lymphoma). PBLs with atypical locations (sellar, infratentorial, intraventricular) were also observed.

Discussion: Because of the varying patterns of MR imaging findings, PBL has a broad differential diagnosis. Primarily, it should be differentiated from GBM and metastases. Furthermore, secondary CNS lymphomas, toxoplasmosis, abscesses, tumefactive demyelinating lesions, vasculitis, and infarcts are also pathological entities that must be considered. Advanced MRI techniques increase MRI specificity and accuracy differentiating, more objectively, PBLs from the aforementioned pathologies. $\mathrm{ADC}$ values, $\mathrm{rCBV}$, time-intensity curves, and metabolites such as lipids can be used to quantitatively characterize PBL. When a hypointense T2 lesion with homogeneous enhancement, low ADC values, no increase of $\mathrm{rCBV}$, and high lipid peaks is found in the deep supratentorial structures, brain lymphoma should be considered as the main diagnosis.

Conclusion: Conventional and advanced MRI techniques can assist in the differentiation of PBLs. Understanding PBL's MR typical and atypical imaging patterns would help to an early diagnosis, thereby guiding to the right preoperative plan and facilitating early treatment of this disease.

\section{1-P56}

EXTREMELY RARE GLIOMA CASES WITH ADVANCED MRI METHODS

VASILEIOS K. KATSAROS $^{1}$, Danai-Eleni Stefanou ${ }^{2}$, Mary Pelechrini $^{2}$, Christos Boskos ${ }^{3}$, George Stranjalis ${ }^{4}$

${ }^{1}$ University of Athens, Athens, Greece, ${ }^{2}$ GAONA "St. Savvas, Athens, Greece, ${ }^{3}$ Athens Medical Center, Athens, Greece, ${ }^{4}$ Evangelismos Hospital, Athens, Greece

\section{Keywords: Glioma, Perfusion, Spectroscopy}

Magnetic Resonance Imaging (MRI) of brain tumours plays a continuous pivotal role of noninvasive pre-treatment evaluation, as well as in the assessment of therapeutic efficacy during post-treatment evaluation. A tumour's features as seen by means of conventional MRI can provide information about its anatomy and indicate its phenotype. Nevertheless, the inclusion of molecular and genetic information as recommended by the WHO 2016 classification has risen a challenge for advanced MRI methods to delineate information over tumour physiology and genotype. Many features provided by structural MRI can contribute in the prediction of the histologic diagnosis of glial tumours, especially when combined with the demographic data, the predisposing factors, the patients' signs and symptoms, as well as the frequency of each glial subtype affecting these categories are taken into account. Because of their extreme rarity and because of them showing features shared with other more common glial neoplasms, tumors of low incidence are prone to misdiagnosis and sets an arduous path in terms of recognition and classification but also in the subsequent management.

This presentation aims to demonstrate extremely rare cases of gliomas and their typical imaging features on conventional MRI and at the same time to highlight the utmost importance of advanced MRI methods in the 
evaluation of tumour phenotype, tumour genotype and post-treatment imaging assessment and monitoring.
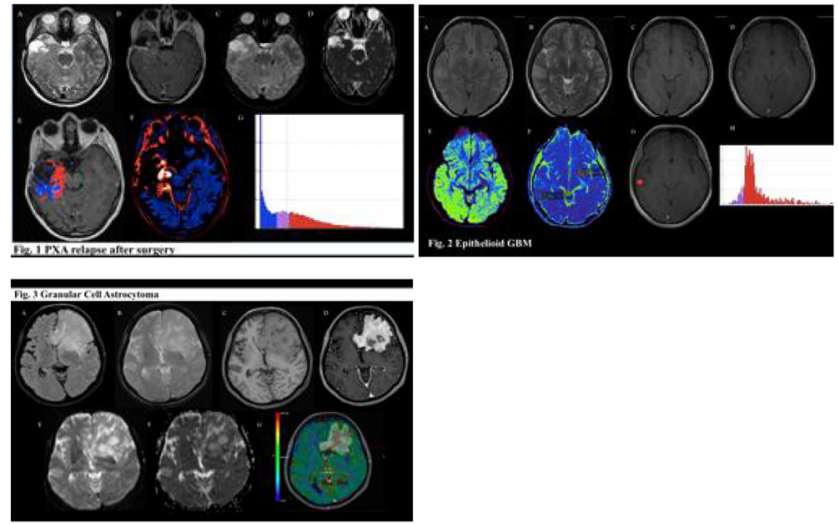

1-P57

CORRELATION OF WADA TEST PERFORMED WITH PROPOFOL AND FMRI OF LANGUAGE AND MEMORY

VASILEIOS K. KATSAROS ${ }^{1}$, Danai-Eleni Stefanou ${ }^{2}$, Maria Pelechrini ${ }^{2}$, Theodoros Kratimenos ${ }^{3}$, Dimitrios Tomais ${ }^{3}$, Dimosthenis Farsaris $^{3}$, Anastasia Verentzioti ${ }^{3}$, Athanasia Alexoudi ${ }^{3}$, Maria Stefanatou $^{3}$, Stephanos Korfias ${ }^{3}$, Stylianos Gatzonis ${ }^{3}$

${ }^{1}$ University of Athens, Athens, Greece, ${ }^{2}$ General Anti-cancer and Onncological Hospital, "St. Savvas", Athens, Greece, ${ }^{3}$ General Hospital of Athens, Evangelismos, Athens, Greece

\section{Keywords: fMRI, Wada test, propofol}

Aim \& Introduction: Temporal lobe epilepsy (TLE) is one of the main pathological entities for temporal lobectomy excising part of the amygdale and hippocampus. However, memory and language can be affected if this procedure is performed on the dominant hemisphere and patients with TLE who are candidates for temporal lobe resection are at risk of postsurgical language and/or memory impairment. The Wada test is the gold standard method for lateralizing language and memory function prior to brain surgery. The commonest agent used for the Wada test is sodium amytal due to its low toxicity, high efficacy, and adequate duration of action but due to the lack of shortage and some other limitations of this method, recent studies have shown that an alternate agent must be suggested and that functional magnetic resonance imaging (fMRI) may be of value for pre-surgical assessment of language lateralization noninvasively. Herein, the purpose of this study is to evaluate the concordance rate between Wada test performed with propofol (PF) and FMRI for lateralizing language and memory.

Materials \& Methods: We performed presurgical fMRI in 8 patients with TLE eligible for surgery. Also, pre-surgical a Wada test was performed in 11 patients by the interventional radiologists and PF was chosen as an alternative, based on its efficacy, safety profile and feasibility, injecting $10 \mathrm{mg}$ in the internal carotid artery. Right hemispheric injection was followed by left hemisphere injection and neuropsychological tests by the clinical neurologists were carried out for localization of memory and language. The post-surgical results were analyzed and the discordant rate was estimated.

Results: 9 out of eleven patients (mean age: 40.8 years with an average disease time of 21 years) underwent surgical excision. The average PF volume of injection was $7.3 \mathrm{ml}$ with no adverse events or allergic reaction during the infusion, except mild transient dizziness in one patient. Postoperatively, language or memory deficit was not observed. fMRI study showed $80 \%$ correlation rate with Wada test.

Conclusion: This study provides further clarification regarding Wada/fMRI concordance, as well as efficacy and safety of propofol as an alternative agent substituting sodium amytal.
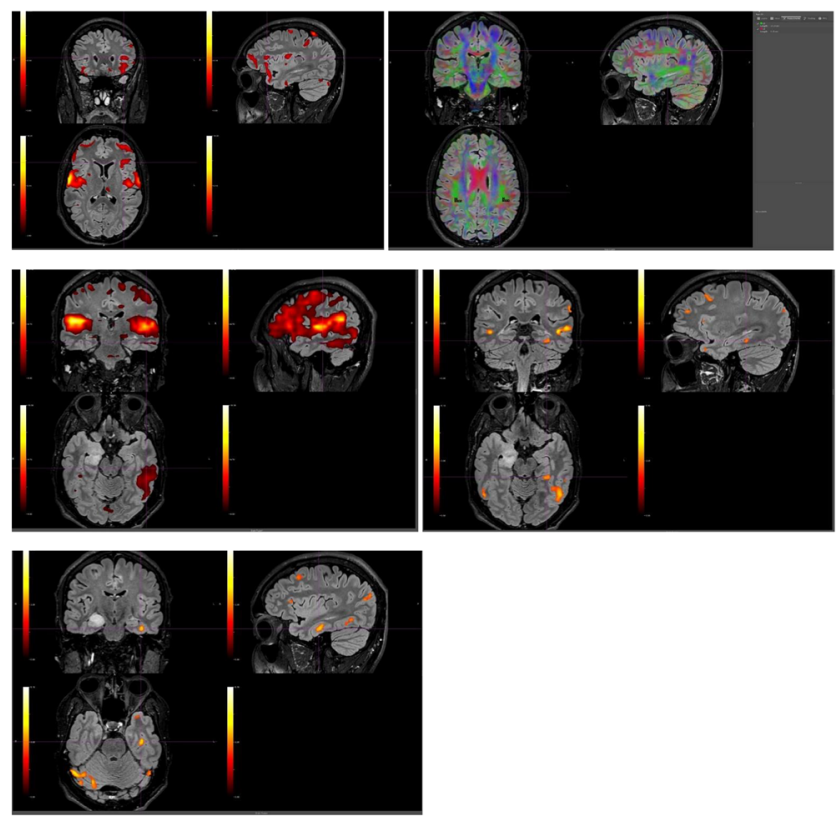

1-P58

ULTRA-HIGH RESOLUTION COMPRESSED SENSING TOF-MRA FOR INTRACRANIAL ANEURYSMS

PATRICK THURNER $^{1}$, Hakim Shakir Husain ${ }^{1}$, Markus Klarhoefer ${ }^{2}$, Bence Nemeth $^{1}$, Jawid Madjidyar ${ }^{1}$, Zsolt Kulcsar ${ }^{1}$, Tilman Schubert ${ }^{1}$

${ }^{1}$ Department of Neuroradiology, University Hospital Zurich, Zurich, Switzerland, ${ }^{2}$ Siemens Healthineers, Erlangen, Germany

Keywords: MR-angiography, compressed sensing, high resolution, intracranial aneurysms

Introduction: 3D time of flight (TOF) MRA shows a high sensitivity for the detection of intracranial aneurysms (1). At 3T, this modality is also helpful in planning of endovascular aneurysm treatment. However, the performance in complex or very small aneurysms is still inferior to DSA due to limited spatial resolution (2). In this study, we used compressed sensing (CS) reconstruction to achieve a higher resolution without increasing scan time and compared the diagnostic performance with conventional TOF-MRA and 3D-DSA.

Methods: 16 patients with conventional TOF at 3T, CS-TOF at 3T and 3D-DSA were included in this retrospective study. Informed consent was obtained. Average inplane resolution of conventional TOF was $0.6 \mathrm{x}$ $0.6 \mathrm{~mm}$ (matrix: $320 / 280$, FOV 190/166) with a slice thickness of $0.6 \mathrm{~mm}$. Inplane resolution of CS-TOF was $0.4 \times 0.4 \mathrm{~mm}$ (matrix 544/ 494 (FOV: 220/200) with a slice thickness of $0.3 \mathrm{~mm}$. Image quality of TOF and and CS-TOF were graded on a four-point scale (excellent / good / sufficient / poor). All MRI scans and 3D-DSAs were independently evaluated for the number of aneurysms. Maximum and minimum aneurysm diameter, aneurysm depth and maximum neck width were measured 
on multiplanar reconstructions. 2-sided, paired t-test were applied to test for statistically significant differences.

Results: 22 aneurysms were detected on 3D-DSA, 22 on CS-TOF and 20 aneurysms on conventional TOF. All TOF and CS-TOF acquisitions were of excellent or good image quality. Average measurement results in $\mathrm{mm}$ for 3D-DSA, CS-TOF and conventional TOF are summarized in table 1. P-values of the performed student t-tests are included in table 1. Maximum neck width measurements of CS-TOF and 3D-DSA were statistically significantly different, all other measurements showed no significant differences.

Discussion \& Conclusion: The diagnostic performance of ultra-high resolution CS-TOF was excellent reflected by the correct depiction of all aneurysms that were found on 3D-DSA. In conventional TOF, two aneurysms were not depicted although image quality was excellent or good for all exams.

One was a very small inferior wall of internal carotid artery aneurysm adjacent to a larger aneurysm (Fig. 1). The other one was a recurrent aneurysm next to a coil pack and microsurgical clip. Conversely, the only significantly different measurement result was maximum neck width between CS-TOF and 3D-DSA.

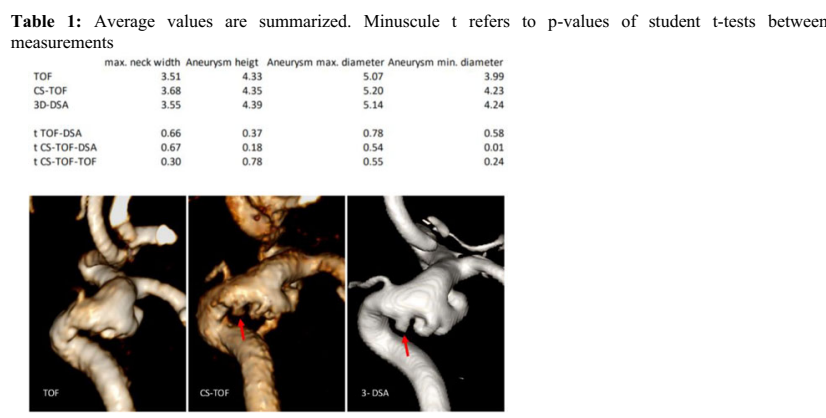

\section{Literature:}

1) Li et al, Accurate Diagnosis of Small Cerebral Aneurysms $5 \mathrm{~mm}$ in Diameter with 3.0-T MR Angiography, Radiology 2014

2) Chen et al, The clinical value of MRA at 3.0 T for the diagnosis and therapeutic planning of patients with subarachnoid haemorrhage, Eur Radiol 2012

\section{1-P59}

CORRELATION OF MR-PERFUSION BASED CEREBRAL BLOOD FLOW (CBF) MEASUREMENTS WITH PHASE CONTRAST MRI BASED CBF MEASUREMENTS IN ISCHEMIC STROKE PATIENTS

BENCE NEMETH ${ }^{1}$, Patrick Thurner ${ }^{1}$, Leyla Acu ${ }^{1}$, Andreas Luft ${ }^{2}$, Susanne Wegener ${ }^{2}$, Jeremia Held ${ }^{2}$, Giuseppe Esposito ${ }^{3}$, Lars Michels ${ }^{1}$, Zsolt Kulcsar $^{1}$, Tilman Schubert ${ }^{1}$

${ }^{1}$ Department of Neuroradiology, Clinical Neuroscience Center, University Hospital Zurich, University of Zurich, Zurich, Switzerland, ${ }^{2}$ Department of Neurology, Clinical Neuroscience Center, University Hospital Zurich, University of Zurich, Zurich, Switzerland, Department of Neurosurgery, Clinical Neuroscience Center, University Hospital Zurich, University of Zurich, Zurich, Switzerland

Keywords: stroke, MR perfusion imaging, phase contrast MRI, cerebral blood flow
Introduction: Magnetic resonance perfusion imaging is able to provide quantitative cerebral blood flow measurements using convolutional postprocessing algorithms (1). Using this method, blood flow is measured on a microvascular level but can be summed up to blood flow of presegmented brain regions. 2D phase-contrast (PC) magnetic resonance imaging, in contrast, measures blood flow of the efferent arteries on a macrovascular level.

In this study, we compare hemispheric blood flow measurements obtained with MR DSC perfusion to those obtained with phase contrast MRI in order to evaluate the correlation of both noninvasive cerebral blood flow measurements.

Methods: 18 patients were prospectively included in this study. MR imaging was performed on a $3 \mathrm{~T}$ scanner. DSC Perfusion imaging was performed using an echo planar imaging sequence (Matrix: 128/128, FOV: 220/220, 5mm slice thickness, TE/TR: 35/2040).

Cerebral blood flow maps were computed using Olea Sphere's Perfusion MRI oSVD algorithm (OLEA, La Ciotat, France).

A commercially available software (NOVA, VasSol, USA) was used to perform phase contrast measurements in the anterior cerebral artery A2 segment (ACA2), middle cerebral artery M1 segment (MCA), posterior cerebral artery P2 segment (PCA).

Brains were segmented into hemispheres using FreeSurfer. Coregistration of 3D T1-weighted imaging and Perfusion imaging was performed using FMRIB's Linear Image Registration Tool (FLIRT). Based on the segmentation, hemispheric $\mathrm{CBF}$ was computed (figure 1).

In order to summarize hemispheric flow in PC-imaging, a summation of PC measurements in the ACA2, M1 and P2-segments was calculated for each hemisphere for each patient.

Pearson correlation was performed to examine the interaction between hemispheric CBF measurements based on PC-MRI and perfusion MRI.

Results: Mean values of perfusion based CBF per hemisphere were 218 $\mathrm{ml} / \mathrm{min}$ (std:106.2), Mean values of phase-contrast based CBF per hemisphere were $272.6 \mathrm{ml} / \mathrm{min}$ (std: 162.4 ).

Hemispheric CBF measurements based on perfusion imaging ( $\mathrm{x}$-axis) are plotted vs. hemispheric CBF measurements based on PC-MRI (y-axis) in figure 2 .

Pearson correlation showed a significant correlation between the CBF measurements $(\mathrm{r}=0.546, \mathrm{p}<0.0001)$.

Discussion: To our knowledge, this is the first attempt to measure hemispheric blood flow using MR-perfusion in humans (2). CBF measurement is a challenge for both invasive and noninvasive imaging. The advantage of MRI for CBF measurements is the capability to measure flow on a macrovascular (phase contrast) and microvascular (perfusion) level. In this work, we could could prove that a moderate correlation between $\mathrm{PC}$ based and perfusion based $\mathrm{CBF}$ measurements exist.

\section{Literature:}

1) Calamante F, Arterial input function in perfusion MRI: A comprehensive review. Progress in Nuclear Magnetic Resonance Spectroscopy, Volume 74, October 2013, Pages 1-32

2) Jeong $Y$ et al, Absolute quantitative MR perfusion and comparison against stable-isotope microspheres. Magn Reson Med 2019 Jun;81(6):3567-3577. doi: 10.1002/mrm.27669. 

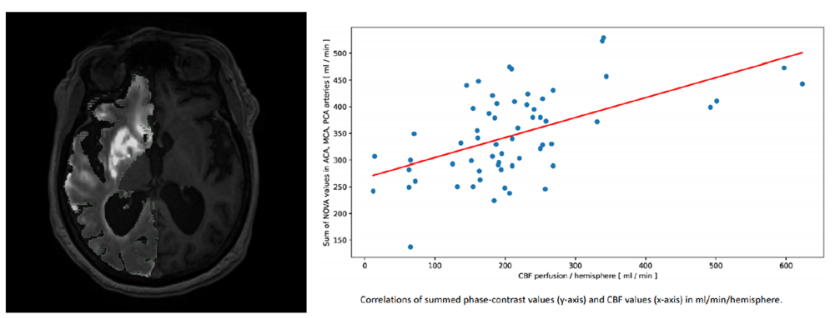

1-P60

MULTIPLE CRANIAL NERVE PALSY ASSOCIATED WITH REVERSIBLE BRAINSTEM LESION IN HASHIMOTO'S ENCEPHALITIS

NURUL HAFIDZAH RAHIM ${ }^{1}$, Nur Fazdlin Abdul Rahim ${ }^{1}$, Norzaini Rose Mohd Zain

${ }^{1}$ Hospital Kuala Lumpur, Kuala Lumpur, Malaysia

Keywords: brainstem, autoimmuneencephalitis, hashimotoencephalitis, cranialnervepalsy

Clinical symptomatology of Hashimoto's encephalitis is usually non-specific, frequently reported as seizure, stroke-like events and movement disorder. The MR imaging findings of this autoimmune disease is characterized by transient focal abnormality which may simulate ischemic stroke, tumour or granulomas, infective process including prion disease or even a neurodegenerative process. The pathogenesis of Hashimoto encephalitis is still unclear, however due to good response to steroid treatment, it may supports vasculitis mechanism. We herein present a case of 23-year old gentleman who is a known case of hypothyroidism presented with 3rd, 4th, 5th, 6th and 7th cranial nerve palsy and left hemiparesis, elevated antithyroid antibody levels and MRI changes of focal unilateral brainstem encephalitis predominantly involving the midbrain and affecting multiple cranial nerve nucleus and tracts. Serial MRI shows resolving brainstem lesion that significantly reduces after steroid therapy accompanied by focal atrophy changes. It is also supported by improvement of clinical symptoms and reducing in trend in antithyroid antibodies titre.
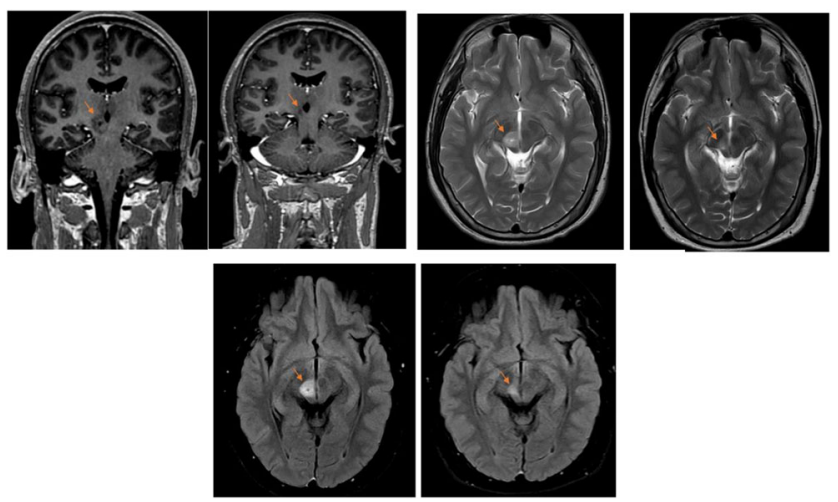

\section{1-P61}

A STAR ON THE BRAIN MRI! T2* AND SWI APPLICATIONS: INITIAL CONCEPTS, TECHNIQUE AND CLINICAL APPLICATIONS

ALINE ALMEIDA BAPTISTA DE OLIVEIRA SALGADO ${ }^{1}$, Ana Paula Alves Fonseca ${ }^{1}$, Erica Antunes Naves ${ }^{1}$, Karolina Pelegrini Cancela $^{1}$, Rita Cassia Maciel Pincerato ${ }^{1}$
${ }^{1}$ Hospital Samaritano, Sao Paulo, Brasil

Keywords: T2*, SWI, brain MRI

Introduction: This exhibit aim to discuss the principles and techniques of gradient-echo (GRE) sequence with $\mathrm{T} 2 *$ based contrast and susceptibility-weighted imaging (SWI) recognizing the main imaging findings associated with many disorders and clinical application. The GRE sequence are used to depict calcification, iron deposition and hemorrhage in various tissues. SWI uses phases information to demonstrate the magnetic susceptibility differences of the blood and calcifications. The purpose of this exhibit is to detail and added values of $\mathrm{T} 2 *$ and SWI sequences in classification brain pathology and categorize the main differential diagnostics, including vascular, traumatic, tumoral, neuropediatric, inflammatory and infection diseases.

Methods: We reviewed reports from our institution's digital teaching files of MRI brains pathologies, focusing in differential imaging patterns based on GRE and SWI sequences and selected some illustrative cases to address diagnostic scenarios of the main important disease in this context.

Results: In our series, based on T2* and SWI sequences we demonstrate some subtype and most differential pathology conditions that can be depicted and categorized by vascular, traumatic, neuropediatric, tumoral, inflammatory and infection diseases.

Discussion: As T2* and SWI sequences has become a widely used clinical tool image offering information about some tissue feature based in intrinsic nature of local magnetic fields to enhance contrast, as hemorrhages, iron deposition, deoxyhemoglobin veins, calcification and microbleeds. The application of T2* and SWI sequences continue expanding and become an important technique that allows detection of many disease patterns involving in most brain pathologies. To characterize the clinical application of T2* and SWI sequences improve an accurate diagnosis.

Conclusion: The added values of T2* and SWI sequences in classification and categorize brain pathology should be included in routine MRI as a red flags that aid and improve accuracy the diagnosis of many different disease patterns.

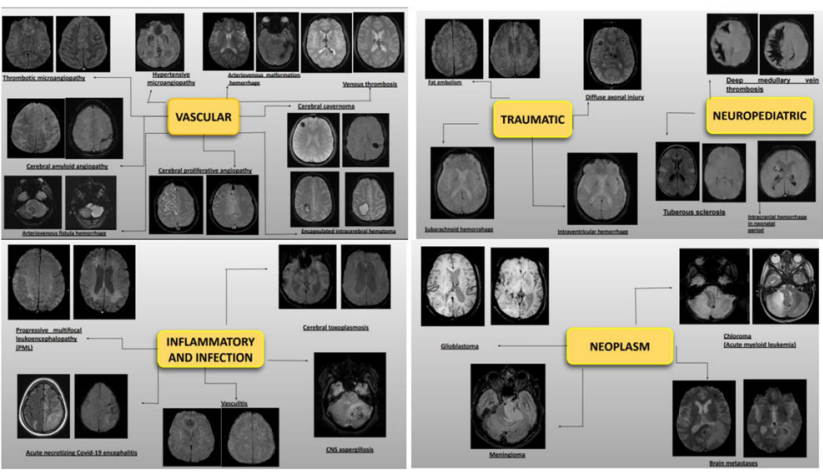

1-P62

UREMIC ENCEPHALOPATHY: A RARE CONDITION WITH RARE SPECIFIC IMAGING FINDINGS

GONCALO GAMA LOBO ${ }^{1}$, Marta Sequeira ${ }^{2}$, Ana Braz ${ }^{3}$, Teresa Morais $^{1}$, Marcos Veiga ${ }^{1}$, Isabel Fragata ${ }^{1}$, Joao Reis ${ }^{1}$ 
${ }^{1}$ Servico de Neurorradiologia, Hospital de Sao Jose, Centro Hospitalar Universitario Lisboa Central, Lisboa, Portugal, ${ }^{2}$ Servico de Neurologia, Hospital de Santo Antonio dos Capuchos, Centro Hospitalar Universitario Lisboa Central, Lisboa, Portugal, ${ }^{3}$ Centro de Imagiologia, Departamento de Neurorradiologia, Hospital da Luz Setubal, Portugal

Keywords: Uremic Encephalopathy, MR, Renal Failure, Basal ganglia

Introduction: Uremic encephalopathy is a rare condition that arises in the context of acute or chronic renal failure with high levels of blood urea. This reversible clinical condition may present with a variety of symptoms from reduced attention, gait disorders and extrapyramidal movements, to severe states as coma.

Three patterns of imaging findings have been described, namely white matter involvement, cortical or subcortical involvement and basal ganglia involvement. Basal ganglia involvement is uncommon and was described in Asians with diabetes. In this subtype, the lentiform fork sign, despite being rare, is a specific imaging feature.

Methods: We report a case of uremic encephalopathy in a 77-year-old man with basal ganglia involvement, showing specific MR findings.

Results: A Caucasian 77-year-old man with chronic renal failure, under hemodialysis, as well as anemia, type 2 diabetes mellitus and dyslipidemia presented with a two-week history of limb weakness and gait disorder, requiring the use of a wheelchair. At observation, marked bradykinesia and freezing of gait were noted.

Laboratory data revealed high values of blood urea - $108 \mathrm{mg} / \mathrm{dL}$ (18$55 \mathrm{mg} / \mathrm{dL}$ ) - with no other significant changes.

Brain MR revealed extensive high T2/FLAIR signal in the lateral and medial contours of both putamina - lentiform fork sign - and mild restricted diffusion in both putamina. These findings were attributed to uremic encephalopathy.

After discharge the hemodialysis scheme was adjusted.

Two months later the patient significantly improved, regaining autonomous gait, without external support, and only with mild bradykinesia.

The follow-up MR revealed significant an almost complete resolution of the basal ganglia signal changes.

Discussion \& Conclusion: Uremic encephalopathy is an uncommon reversible entity with potentially severe consequences. The specific imaging findings are extremely rare, but if present in the right clinical and laboratory context can be of great diagnostic value. This case illustrates the lentiform fork sign, a rare but specific imaging sign, as well as its reversibility with the adequate treatment.

\section{1-P63}

\section{CEREBRAL CT PERFUSION: UNMASKING STROKE MIMICS}

DAVID CASTANEDO VAZQUEZ ${ }^{1}$, Marta Drake-Perez ${ }^{1}$, Dario Herran de la Gala ${ }^{1}$, Teresa Cobo Ruiz ${ }^{1}$, Maria Jose Galante Mulki ${ }^{1}$, Javier Garcia Poza $^{1}$, Enrique Marco de Lucas ${ }^{1}$

${ }^{1}$ Radiology Service at the "Marques de Valdecilla" University Hospital, Santander, Spain

Keywords: CT perfusion, Stroke mimics, Cerebral ischemia, Brain tumor, Epilepsy

Introduction: $\mathrm{CT}$ perfusion (CTP) enables the identification of ischemic brain tissue in patients with ischemic stroke, differentiating between brain regions with high probability of irreversible infarction (core) and areas at risk of infarction (penumbra). Estimation of brain tissue viability is paramount in patient selection for intra-arterial thrombectomy.

However, tissue viability estimation is challenging and prone to error, as perfusion alterations may happen in the setting of crossed cerebellar diaschisis, chronic infarcts and in patients with anatomic variants of the circle of Willis.

Perfusion imaging abnormalities have also been reported of added value for identifying stroke mimics, such as glial tumors, seizures, migraine attacks, encephalitis, reperfusion syndromes, PRES syndrome, reversible cerebral vasoconstriction syndrome, migraine attacks, venous thrombosis or hypoglycemia, among others.

The main purpose of this work is to analyze perfusion alterations associated with stroke mimics.

Methods: We examined discordant perfusion abnormalities in patients presenting with acute focal neurologic symptoms presumed for stroke, who underwent CT angiography (CTA) of the head and neck and CTP at a tertiary care center in Santander (Spain), comparing patients with stroke mimic to those with stroke.

The most likely diagnosis was determined by the treating clinician and follow - up imaging studies.

\section{Results:}

- Many underlying physiologic conditions and anatomic variants may lead to the appearance of ischemic penumbra at CTP.

- Various of the aforementioned stroke mimics present in CTP with a pattern of hyperperfusion, which can also be seen in acute ischemic stroke, in the setting of a phenomenon called luxury perfusion.

- Abnormal Tmax findings were much more likely to be discordant with clinical symptoms and CTA findings.

- An entirely discordant penumbral pattern was strongly associated with the diagnosis of mimics.

Discussion \& Conclusions: CTP plays a key well established role in the diagnosis of ischemic stroke. Thrombolytic therapy should be avoided if there is doubt about the potential benefits of therapy. Stroke mimics should be considered before a definitive diagnosis of ischemic penumbra is made and thrombolytic treatment is initiated. When the patient's symptoms are discordant with the finding of ischemic penumbra, all available radiologic images should be carefully reviewed and correlated with the clinical history to allow the detection of mimics.

\section{1-P64}

MULTIMODAL ASSESSMENT DURAL ARTERIOVENOUS FISTULAS

\section{ANISH SURESHKUMAR ${ }^{1}$, Shan $^{\mathrm{Li}^{1}}{ }^{1}$, Angelica Patino ${ }^{1}$}

${ }^{1}$ Radiology, Baystate Medical Center-UMMS, USA

Keywords: Dural, AVF, Intracranial, spinal, Cognard, Borden

Introduction: Dural Arteriovenous Fistulas (dAVFs) are a group of complex entities the can cause significant intracranial or even spinal pathology. The underlying pathology is related to formation of arteriovenous shunts with dural vessels and form a minor but significant portion of vascular malformations.

dAVF clearly shows the interplay between specialities, such as radiology, interventional radiology and surgery, to help diagnose and treat patients. Many different modalities are used to diagnose the disease, such as MRA, CTA and angiography, to diagnose dAVF. Description and classification 
is aided by multiple classification schemes such as Cognard and Borden. These classifications in turn helps predict the complication rate, such as inter cranial hemorrhage and venous hypertension, and the need for intervention and the type of intervention.

dAVFs can be especially difficult to accurately diagnose for radiologists, especially in training, without adequate prior exposure. The purpose of this educational presentation is to show multiple cases with various presentation and the methods used to diagnose. Complications are also shown as patient might present with complication as the first sign. It is important to accurately diagnose this pathology to avoid severe morbidity.

Methods \& Results: This education poster will show multiple cases of inter cranial and spinal dAVF along with patient presentations, the modalities used for diagnosis and type of interventions performed. The cases are organized into the Cognard and/or Borden classifications.

Discussion \& Conclusion: dAVFs, intracranial or spinal, can be benign process or can have devastating sequelae if not accurately diagnosed and properly treated. The aim for this poster is to help understand the presentation, the diagnosis and treatment of this entity with multiple variations. The management of dAVF spans multiple specialties, and requires proper coordination to achieve the best outcomes.
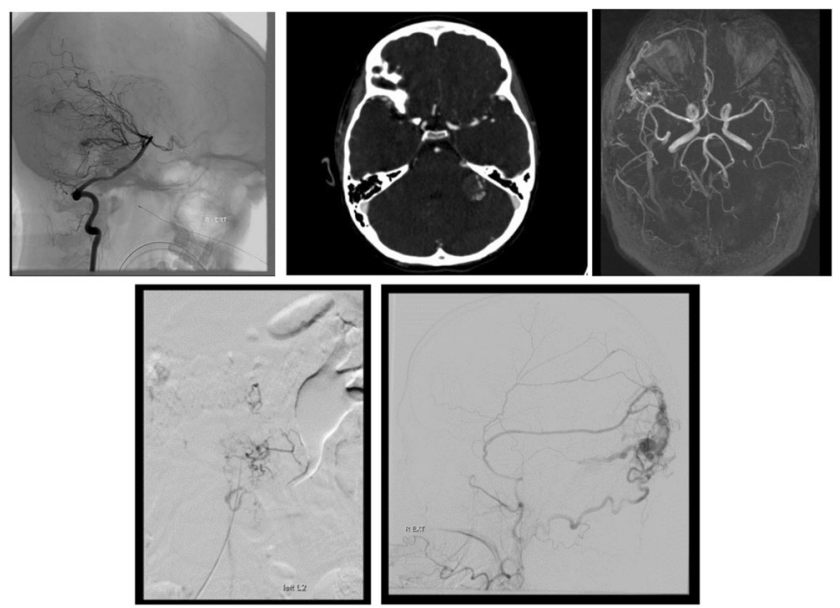

\section{1-P65}

PHOTOREALISTIC DEMONSTRATION OF BRAIN SURFACE CORTICAL ANATOMY USING CINEMATIC RENDERING OF VOLUMETRIC MRI DATA

\section{$\frac{\text { GERARD DEIB }^{1}}{\text { Abdul Tarabishy }}{ }^{1}$ Ryan Brotman ${ }^{1}$, Dharia Lakhani ${ }^{1}$, Hal Meltzer ${ }^{1}$, \\ ${ }^{1}$ Department of Neuroradiology, West Virginia University}

Keywords: Cortical Anatomy, Cinematic Rendering, MRI, Novel Technology, Education and Communication

Introduction: A comprehensive understanding of an individual patient's cortical surface anatomy requires an appreciation of the layered architecture of the overlying scalp, skull, dura and blood vessels as well as an appreciation of the convoluted relationship between the various sulci and gyri. Neurosurgeons and neuroradiologists need to understand and conceptualize this anatomy in a format that depicts the relative proximity of adjacent structures in all dimensions and degrees of freedom. Technology that improves visualization of these complex anatomic structures and their spatial relationship would significantly improve student, trainee and physician knowledge and consequently patient outcomes. A new rendering algorithm, Cinematic Rendering (CR),1,2 incorporates a complex lightening model that allows for the creation of photorealistic models from the reconstruction of isotropic 3D imaging data. 3,4 CR allows for strikingly detailed imaging depictions of anatomic structures and an unprecedented display of their 3D anatomic relationships. Whilst this algorithm has mostly been used to depict CT data, its use in depicting the brain in MRI is not well documented in the literature.

Methods: We present a series of images depicting brain surface cortical anatomy, in orientations that mimic a surgical approach or cadaveric dissection. These images were derived from isotropic, high resolution volume MRI data. A dedicated application ((Anatomy Education Siemens, Munich, Germany)) was utilized to create images and video content.

Results: The user can accentuate the appearance of structures by changing the display settings on the $\mathrm{CR}$ reconstructions. Consequently, visualization of cortex to white matter, brain surface to vessels, subarachnoid space to cortex and skull to intracranial structures can be optimized. Layers of the overlying soft tissue can be progressively removed to provide a comprehensive assessment of the entirety of the region. CR models can be enlarged, rotated and shifted arbitrarily. In the context of the brain surface, there are several benefits to utilizing this technology.5 Complex, small structures can be demonstrated in very high detail. The depth and architecture of the sulci can be better appreciated than on traditional imaging modalities. With appropriate display settings, the relationship of the cortical surface to the adjacent vasculature can be delineated.

Conclusion: This series serves to demonstrate the enhanced display capabilities of CR using MRI data to delineate brain surface cortical anatomy to educate students and trainees and as part of collaborative communication with a multidisciplinary team.

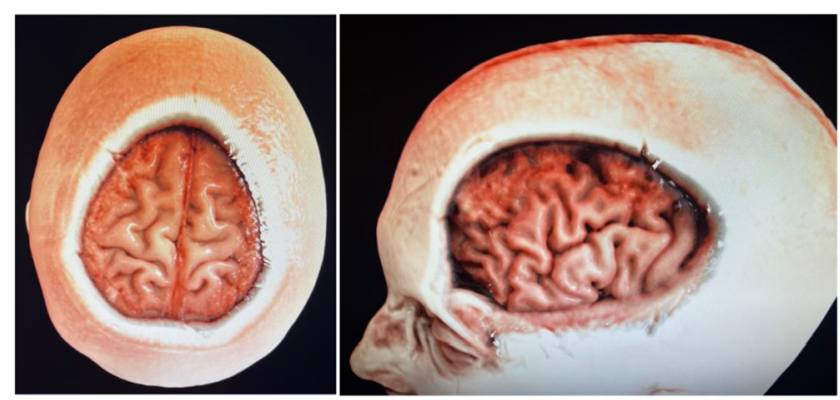

1-P66

IMAGING OF MENINGIOMA WITH SYNTHETICMR

RICCARDO LUDOVICHETTI ${ }^{1}$, Benedicte M. A. Delattre ${ }^{1}$, Jose Manuel Baiao Boto ${ }^{2}$, Daniela Dumitriu La Grange ${ }^{2}$, Torstein R. Meling $^{3}$, Maria Isabel Vargas Gomez ${ }^{2}$

${ }^{1}$ Department of Radiology, Geneva University Hospital, Geneva, Switzerland, ${ }^{2}$ Department of Neuroradiology, Geneva University Hospital, Geneva, Switzerland, ${ }^{3}$ Department of Neurosurgery, Geneva University Hospital, Geneva, Switzerland

Keywords: SyntheticMR, Meningiomas, Quantitative and qualitative imaging

Purpose: The purpose of this study is to characterize meningiomas with the use of SyntheticMR, a novel quantitative and qualitative technique 
Materials \& Methods: This study, approved by our review board ethical committee, was carried out in our hospital using Synthetic imaging in addition to the conventional sequences FSET2, FLAIR, DWI and T1 with gadolinium on 3T MR scanners (Siemens and Philips machines).

35 patients with meningiomas ( 6 males, 29 females; mean age of $61 \pm 17$ years; age range 21-90 years), followed-up in our institution between October 2019 and October 2020 were included.

SyMRI software, Version 8 (SyntheticMR, Linköping, Sweden) was used to generate $\mathrm{T} 1, \mathrm{~T} 2$, and proton-density quantitative maps (Figure 1). OsirixMD was then used to measure quantitative values of T1, T2 and PD using an ROI. Descriptive statistics, independent samples T-test with Levene's test for equality of variances and Pearson correlation were applied.

Results: We analyzed 42 meningiomas from 35 patients, 8 of which were associated with edema and 5 contained calcifications. Mean values of signal intensities of meningiomas in Synthetic T1, T2 and PD at 3T MRI were $1382.6 \pm 391.7 \mathrm{~ms}, 95.6 \pm 36.5 \mathrm{~ms}$ and $89.1 \pm 9.7 \mathrm{pu}$, respectively. Signal intensities did not differ significantly between meningiomas with and without edema ( $p=0.994, p=0.356$ and $p=0.221$, respectively) or between meningiomas containing and not containing calcifications $(\mathrm{p}$ $=0.840, p=0.710$ and $p=0.455$, respectively). $\mathrm{T} 1$ and $\mathrm{T} 2$ measured in the normal appearing white matter and in meningiomas of the patients included in our study roughly approximated reference values found in the literature. T1, T2 and DP values of normal appearing white matter and meningiomas were significantly different $(\mathrm{p}<0.001)$.

Conclusions: Synthetic imaging is a valid option for a quantitative and qualitative characterization of meningiomas.

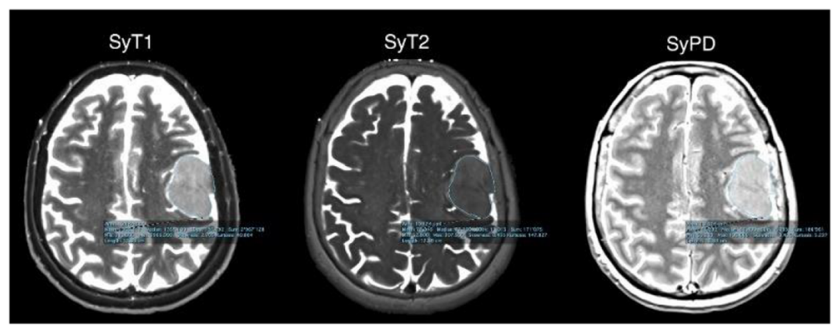

\section{INTERVENTIONAL}

\section{2-P1}

DUAL ENERGY CT ASSESSMENT FOLLOWING MECHANICAL THROMBECTOMY IN ACUTE ISCHAEMIC STROKE: A REVIEW

SONAL SACHDEV $^{1}$, Julian Maingard ${ }^{2,3,4}$, Ariel Dahan ${ }^{3}$, Hong Kuan Kok $^{4,5}$, Christen D. Barras ${ }^{6,7}$, Winston Chong ${ }^{2,8,9}$, Hamed Asadi ${ }^{2,3,4,9,10,11}$

${ }^{1}$ Department of Neurosurgery, Austin Health, Melbourne, Australia, ${ }^{2}$ NeuroInterventional Radiology Unit, Monash Imaging, Monash Health, Melbourne, Australia, ${ }^{3}$ Interventional Neuroradiology Service, Austin Health, Melbourne, Australia, ${ }^{4}$ School of Medicine, Deakin University, Waurn Ponds, Geelong, Australia, ${ }^{5}$ Interventional Radiology, Northern Health, Melbourne, Australia, ${ }^{6}$ South Australian Health and Medical Research Institute, Adelaide, South Australia, Australia, ${ }^{7}$ University of Adelaide, Adelaide, South Australia, Australia, ${ }^{8}$ Faculty of Medicine, Nursing and Health Sciences, Monash University, Clayton, Australia, ${ }^{9}$ Monash Imaging, Monash University, Melbourne, Australia, ${ }^{10}$ Interventional Neuroradiology Service, St Vincent's Hospital, Melbourne, Australia, ${ }^{11}$ Florey Institute of Neuroscience and Mental Health, The University of Melbourne, Parkville, Australia
Keywords: Dual energy CT, Stroke, Thrombectomy Acute ischaemic stroke (AIS) is a leading cause of morbidity and mortality worldwide. The first-line treatment for AIS due to acute large vessel occlusion is endovascular mechanical thrombectomy (MT), with intravenous (IV) thrombolysis in select cases (1). Iodinated contrast agents used during MT can often cross the damaged blood brain barrier in the AIS setting, and remain in the brain parenchyma following the procedure. This so called 'contrast-staining' can mimic intracerebral haemorrhage (ICH) or mask further infarcts on routine follow-up CT, possibly leading to inappropriate clinical management decisions.

Dual energy CT (DECT), which involves simultaneous acquisition of CT images with two different tube voltages, has been shown in several small studies to improve differentiation of haemorrhage from contrast extravasation following MT. We performed a literature search to evaluate the utility of DECT in the early post-MT period in AIS. A total of 827 patients were included across 11 studies. The sensitivity of DECT in detection of haemorrhage versus contrast extravasation was $95 \%$ to $100 \%$, and the specificity ranged from $68 \%$ to $91 \%$.

DECT may provide important prognostic information in the immediate post-intervention period in AIS, with no additional morbidity and, depending on the DECT protocol used, negligible or no additional radiation dose to the patient (2). Consideration to imaging protocols involving postMT DECT should be given in practices where this technology is available. This would likely result in more judicious use of critical care monitoring, guide appropriate post-MT antiplatelet and anticoagulant use, and allow early onward stroke rehabilitation.

2-P2

TRANSRADIAL DIAGNOSTIC CEREBRAL ANGIOGRAPHY: IS PROPHYLACTIC INTRA-ARTERIAL NITROGLYCERIN NECESSARY?

\section{$\underline{\text { LAU HOI TO }}^{1}$, Yin Lun Edward Chu ${ }^{1}$, Lee Raymand ${ }^{1}$}

${ }^{1}$ Department of Radiology, Queen Mary Hospital, Hong Kong

Keywords: Transradial, DSA, Nitroglycerine, vasospasm

Introduction: Trans-radial approach (TRA) cerebral angiography has gained popularity in recent times. Various studies had demonstrated its superiority over traditional trans-femoral approach (TFA) in terms of lower access site and improved patient's comfort. Vasospasm after radial puncture decreases procedural success rate and leads to patient discomfort. Nitroglycerin is the key component of the so called "radial cocktail" which is commonly administered intra-arterially following radial access. Its primary aim is to reduced vasospasm. Niroglycerin is a potent vasodilator, but it also has numerous adverse effects and contraindications. From our experience, some patients suffered from significant blood pressure drop after intra-arterial nitroglycerin administration during TRA cerebral angiography, which posed risk of stroke to patient.

Recent trials from cardiac catheterization have shown that prophylactic use of nitroglycerin offered no advantage in terms of patient comfort and its omission did not cause any objective difficulty to operators. To the best of our knowledge, no similar study has been reported by the neurointervention community.

Materials \& Methods: Between September 2020 and June 2020, 43 patients underwent TRA diagnostic cerebral angiography without prophylactic intra-arterial nitroglycerin in our centre. We retrospectively evaluated all these patients in our institution. TRA cerebral interventional procedures were excluded.

Patient demographics, clinical information, radial artery diameter, diagnostic catheters used, successful TRA access, supra-aortic and branch 
vessel catheterization, radial access site-related complications, and total procedural time were recorded.

Results: Overall, the transradial access success rate $(97.7 \%)$, femoral crossover rate $(2.3 \%)$, TRA cerebral angiography success rate $(100 \%)$ and radial access site-related complication rate (4.7\%) were all comparable to conventional TRA cerebral angiography with nitroglycerin. In particular, only one case encountered intra-procedural radial artery spasm which was adequately treated with additional verapamil.

Conclusion: The results of our study suggest that transradial diagnostic cerebral angiography could be performed without prophylactic nitroglycerin. Omission of this vasodilator would not cause significant increase in rate of vasospasm, radial access site-related complication, or femoral crossover rate

\section{2-P3}

CONCOMITANCE OF TWO BRAIN AVMS AND A DAVF WITH A COMMON VEINOUS DRAINAGE. CASE REPORT AND HYPOTHESIS OF ETIO-PATHOGENESIS

ANDREA ROSI ${ }^{1}$, Gianmarco Bernava ${ }^{1}$, Jeremy Hofmeister ${ }^{1}$, Torstein R. Meling ${ }^{2}$, Karl-Olof Loevblad ${ }^{1}$, Paolo Machi ${ }^{1}$

${ }^{1}$ Division of Diagnostic and Interventional Neuroradiology, Department of Diagnostics, Geneva University Hospitals, Geneva, Switzerland, ${ }^{2}$ Division of Neurosurgery, Department of Clinical Neurosciences, Geneva University Hospitals, Geneva, Switzerland

Keywords: AVM, DAVF, etio-pathogenesis, cerebral hemodynamic

Introduction: Intracranial arteriovenous shunts (icAVS) are rare lesions resulting from a pathological communication between arteries and veins located in the brain itself or in the surrounding structures. Arteriovenous malformations of the brain (bAVMs) and cranial dural arteriovenous fistulas (cDAVFs) are two types of icAVS with different anatomopathologic and etio-pathogenetic features. DAVFs are distinguished from bAVMs by:

- localization and arteries: dural localization supplied by dural arteries for DAVFs and parenchymal localization supplied by pial arteries for AVMs; - the absence of a real parenchymal "nidus";

- origin: congenital in bAVMs and acquired in DAVFs, with very rare exceptions;

- frequency between icAVS: AVM 85-90\% VS DAVFs 10-15\%

The pathogenesis of DAVFs involves thrombosis and venous hypertension as trigger factors, usually following a trauma or an inflammatory stimulus.

The presence of multiple bAVMs is rare (about $2 \%$ of cases) and mostly associated with syndromes. The concomitance of AVMs and DAVFs has been described in only a few case reports, mainly at the spinal level.

Methods: The present study describes the clinical presentation and the neuroimaging of the case of a 58-year-old man with two bAVMs and a cDAVF, focusing on neuroanatomical and hemodynamic features observed on angiography and MRI. A literature search was performed to obtain possible etio-pathogenic hypotheses to explain this very rare association.

Results: A non-syndromic patient with a right parieto-occipital subarachnoid hemorrhage was found to have two Spetzler-Martin 1 bAVMs in the superficial temporo-occipital and parieto-occipital sites from the right hemispheric convexity, respectively. The main venous drainages of the two bAVMs reached the middle third of the right transverse sinus at the level of a stenosis determined by a Pacchioni granulation. At this exact point, there was a Cognard type III cDAVF with the point of fistula at the junction of a right basal temporo-occipital vein and the right transverse sinus.

Discussion \& Conclusion: On the basis of the literature search, we discuss the pathophysiological link between bAVM and cDAVF taking into account the rarity of the DAVF, the uncommon association between bAVM and cDAVF, and the venous hypertension created by the venous drainages of the congenital AVMs towards a stenosed segment of a dural sinus. The localized venous hypertension could lead to the development of an acquired cDAVF through at least two different mechanisms, namely by promoting the local production of angiogenetic factors and metalloproteinases and by inducing the opening of dural arteriovenous microshunts, as described in literature.
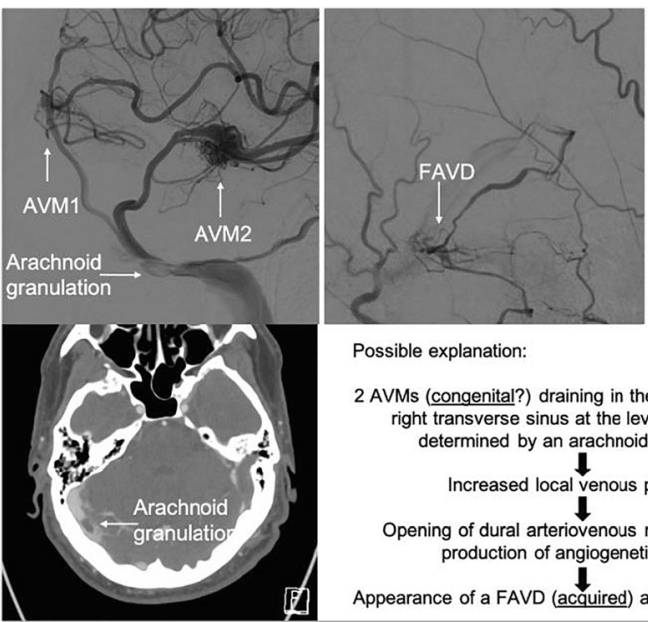

Possible explanation

2 AVMs (congenital?) draining in the same point on the right transverse sinus at the level of a stenose determined by an arachnoid granulation etermined by an arachnoid granulation Increased local venous pressure

Opening of dural arteriovenous microshunts and production of angiogenetic factors

\section{2-P4}

MRI-GUIDED LASER INTERSTITIAL THERMAL THERAPY (MRG-LITT) PRECEDED B Y INTRAOPERATIVE NEURONAVIGATION FOR MALIGNANT BRAIN TUMOURS - A SINGLE-CENTER CASE SERIES

VASCO SOUSA ABREU ${ }^{1}$, Luis Botelho ${ }^{1}$, Filipe Vaz da Silva ${ }^{2}$, Vasco Sa Pinto $^{2}$, Carla Silva ${ }^{2}$, Eduardo Procaci ${ }^{2}$, Joao Xavier ${ }^{1}$

${ }^{1}$ Department of Neuroradiology, Centro Hospitalar Universitario do Porto, Porto, Portugal, ${ }^{2}$ Department of Neurosurgery, Centro Hospitalar Universitario do Porto, Porto, Portugal

Keywords: MRI-guided laser interstitial thermal therapy, MRg-LITT, brain metastases, radionecrosis, brain tumors, glioblastoma

Introduction: MRI-guided laser interstitial thermal therapy (MRg-LITT) is a technique that uses a laser device to produce precise and monitored heat damage to achieve selective ablation of a lesion. MRg-LITT is an approved minimal invasive approach, currently used with neuronavigation methods in combination with intraoperative imaging and continuous thermography. Its optimal applications and advantages are gaining scientific support with an already established role for the treatment of gliobastomas, brain metastases and radionecrosis.

Methods: Six cases undergone MRg-LITT, from December 2019 to June 2021, in our institution. Included patients were not candidates for open surgery or radiosurgery treatments, had lesions in eloquent areas or poor functional status. 
Results: Selected patients included three woman and three men and the average age was 60yo (40-76). Four were diagnosed with gliolastoma, one with anaplasic oligodendroglioma, and one with radionecrosis lesion after radiotherapy for a cerebellar peduncle metastasis.

MRg-LITT system was the Visualase apparatus (Visualase, Inc.), including a 980-nm diode continuous laser for thermoablation. After laser placement with intraoperative image neuronavigation, patients were transferred to the MRI-room, where MR-thermometry provided real-time temperature monitoring, allowing the safety and efficacy of the procedure. So far, the patient with anaplasic oligodendroglioma remained progression-free at 15 months' follow-up. One patient with glioblastoma showed initial control up to 4 months but subsequent progression occurred at 8 months, requiring further surgery. The patient with radionecrosis revealed local control of the cerebellar lesion but slight supratentorial progression at 15 months. These three patients maintained their prior clinical status without further neurological deficits. However, one patient with glioblastoma suffered an intraprocedural hemorrhage, worsening the previous deficit, and died one month later due to SARS$\mathrm{CoV}-2$ infection.

The other two glioblastoma cases were recently submitted to MRg-LITT and have not yet completed the 3-month follow-up timepoint; however, the procedure occurred without complications and both patients maintained their clinical status at discharge.

Discussion \& Conclusion: MRg-LITT is a minimally invasive modality available for the treatment of malignant brain tumors. It has encouraging results for functional preservation in difficult to approach lesions or patients who are not candidates for surgery or when radiosurgery is contraindicated. The synergistic effect with additional chemotherapy due to blood-brain barrier disruption should be taken in account as it offers a window to increase progression-free and overall survival. Future prospective trials should further guide the management of this technique, concerning its cost-effectiveness and risk control, in the treatment of brain tumors.

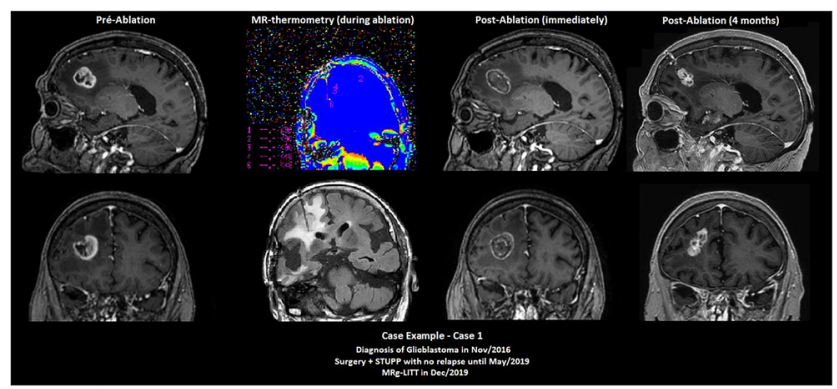

\section{2-P5}

EFFECTIVENESS AND SAFETY OF TICAGRELOR AS AN ALTERNATIVE TO CLOPIDOGREL FOR UNRUPTURED INTRACRANIAL ANEURYSM TREATMENT WITH FLOW DIVERTERS

PEDRO BEM GOMES ${ }^{1}$, Luis Rito $\mathrm{Cruz}^{2}$, Sofia Queiros de Almeida Xavier $^{3}$, Isabel Fragata ${ }^{4}$, Joao Lopes dos Reis ${ }^{4}$

${ }^{1}$ Neuroradiology Department, Unidade Local de Saude de Matosinhos, Matosinhos, Portugal, ${ }^{2}$ Neuroradiology Department, Hospital de Coimbra, Coimbra, Portugal, ${ }^{3}$ Neuroradiology Department, Hospital de Braga, Braga, Portugal, ${ }^{4}$ Neuroradiology Department, Centro Hospitalar Universitario de Lisboa Central, Lisboa Portugal

Keywords: cerebral aneurysm, flow diverter, ticagrelor, clopidogrel
Background: Dual antiplatelet therapy with acetylsalicylic acid (ASA) and a P2Y12-receptor is often used to prevent thrombotic complications after placement of flow diverter devices for cerebral aneurysms. However, clopidogrel resistance frequently occurs and can lead to complications. Ticagrelor is an alternative, not requiring platelet inhibition testing, however there is still need for additional studies on safety and efficacy. We aimed to compare complications and clinical outcome in patients under clopidogrel versus ticagrelor for flow diversion in unruptured cerebral aneurysms.

Methods: We performed a retrospective study from January 2010 to December 2019 that included consecutive patients with unruptured cerebral aneurysms that were treated with flow diverters. No clopidogrel resistance testing was performed. Acute and delayed complications, immediate ischemic stroke and stroke at 90 days, long term (24 month) modified Rankin score (mRS) and the rate of aneurysm exclusion were reviewed from the patients medical records. Non parametric tests were used to compare groups.

Results: Of the total 162 patients treated with flow diverters, $116(71.6 \%)$ were on a regime with clopidogrel and ASA, and 46 (28.4\%) with ticagrelor and ASA. There was no significant difference between the clopidogrel versus ticagrelor group in procedure-related complications ( $22.2 \%$ vs $21.7 \%, \mathrm{p}=0.926)$, and in delayed complications $(6.0 \%$ vs $4.3 \%, \mathrm{p}=0.504$ ). Ischemic stroke events at 90 days were similar between groups $(15.5 \%$ vs $15.2 \%, \mathrm{p}=0.962)$.

Clinical outcomes (mRS 0-2) at 24 months were similar in patients under clopidogrel and ticagrelor $(91.4 \%$ vs. $89.2 \%, p=0.741$ for a $n=130)$, as was the rate of exclusion of the aneurysms $(77.6 \%$ vs $89.5 \%, \mathrm{p}=0.350)$.

Conclusion: Our study is in line with the literature and supports the idea that ticagrelor is safe and effective in replacing clopidogrel in the treatment of unruptured cerebral aneurysms with flow diverters.

Abbreviations: ASA, acetylsalicylic acid; mRS, modified ranking scale.

\section{2-P6}

COMPREHENSIVE EVALUATION OF FACTORS AFFECTING TREMOR RELAPSE AFTER MRGFUS THALAMOTOMY: CASECONTROL STUDY

FEDERICO BRUNO $^{1}$, Emanuele Tommasino ${ }^{1}$, Alessia Catalucci ${ }^{2}$, Alessio Gagliardi ${ }^{1}$, Patrizia Sucapane ${ }^{3}$, Carlo Masciocchi ${ }^{1}$, Alessandra Splendiani ${ }^{1}$

${ }^{1}$ Department of Biotechnological and Applied Clinical Sciences, University of L'Aquila, L'Aquila, Italy, ${ }^{2}$ Neuroradiology and Interventional Radiology, San Salvatore Hospital, L'Aquila, Italy, ${ }^{3}$ Neurology, San Salvatore Hospital, L'Aquila, Italy

Keywords: MRgFUS thalamotomy, Parkinson's disease, essential tremor

Purpose: To evaluate the influence of demographic, clinical, procedural and imaging parameters on clinical outcome in patients with tremor relapse after unilateral MRgFUS Vim thalamotomy, compared to patients with stable outcome after treatment.

Methods: We retrospectively evaluated 79 patients submitted to VIM thalamotomy. From clinical reports we retrieved $11 / 79$ patients with tremor relapse (increase in the Fahn-Tolosa-Martin score $>5$ compared to postprocedural values).

Clinical and demographic evaluation in all patients included underlying pathology (PD/ET), age,gender,disease duration. 
Clinical and demographic characteristics were compared to a control group of patients who did not experienced tremor relapse at the same follow-up intervals.

From procedural reports we retrieved the following procedural data: Skull Density Ratio (SDR), Skull Area (cm2), Accumulated thermal dose (ATD) temperature, Accumulated thermal dose (ATD) area, active Elements, Sonications, Target movements, Maximum Power (W), Maximum Energy $(\mathrm{J})$, maximum $/$ mean temperature $\left({ }^{\circ} \mathrm{C}\right)$, Max sonication duration. For the evaluation of procedural parameters, we selected a control group of patients matched pairwise for age, sex, pathology, years of disease, pre-treatment FTM score and SDR values.

Imaging evaluation included measurement of the thalamotomy lesion size $(\mathrm{mm})$, and lesion location (central/eccentric) respect to the dentorubrothalamic tract, evaluated after DTI tractography reconstruction.

Results: Compared with the con trol groups, in the study group (relapse) we observed a significant longer disease duration, higher target movements number, smaller lesion size at 1 year, and eccentric lesion position rspect to the DRTT.

Conclusion: Lesion size and location, and thus precise Vim targeting, may be the most relevant factor affecting tremor relapse after MRgFUS thalamotomy.

\section{2-P7}

ENDOVASCULAR APPROACH TO ACUTELY RUPTURED INTRACRANIAL ANEURYSM: SHOULD WE USE STENT OR NOT? - UNICENTRIC RETROSPECTIVE COHORT STUDY

DIOGO ANDRE GOMES FERREIRA MARQUES ${ }^{1}$, Ricardo Pimentel Silva $^{1}$, Filipa Maria Neves Castelao ${ }^{1}$, Francisca Sena Batista ${ }^{1}$, Ana Rita da Silva Piteira ${ }^{2}$, Ana Cristina Rodrigues Rios ${ }^{1}$

${ }^{1}$ Hospital Garcia de Orta, Almada, Portugal, ${ }^{2}$ Centro Hospitalar de Setubal, Setubal, Portugal

Keywords: Subarachnoid Hemorrhage, Ruptured Aneurysm, Endovascular Treatment

Introduction: Ruptured intracranial aneurysm is one of the main causes of intracranial hemorrhage with a high mortality and morbidity. Nowadays aneurysms are treated surgically or endovascularly, depending on the patient comorbidities and the imaging features of the aneurysm. In this study the author aimed to analyse the endovascular approach of these patients in our center, comparing the use of coils versus stent (with or without coils) in ruptured aneurysms.

Methods: In this retrospective cohort study, we collected data from 94 adult patients who were admitted in our centre with acute aneurysmal intracranial hemorrhage and were submitted to endovascular treatment between January 2016 and May of 2020. We analysed the risk of vasospasm, new hemorrhage and refractory hydrocephalus, as well as inhospital and 1-year mortality, and the functional outcome [modified Rankin Scale(mRS)] af 1 year.

Results \& Discussion: From the 94 patients analyzed, 64 were female $(68,1 \%) .71(75,5 \%)$ patients were submitted to coiling of the aneurysm, and $23(24,5 \%)$ were submitted to stenting of the aneurysm. The median age of the subjects was 58,94 $( \pm 4)$ years old in the coiling group and 61,43 $( \pm 17,2)$ years old in the stenting group. All our patients had a prehemorrhage $\mathrm{mRS}$ between $0-3$, without a statistically significant difference between both groups $(p=0,102)$. At admission, almost half of all patients had a World Federation of Neurosurgical Societies (WFNS) score of 1, with no difference between the groups $(p=0,148)$. There was a statistically significant difference between groups regarding the presence of residual aneurysm $[33,8 \%$ in coiling group versus $65,2 \%$ in stenting group; $\mathrm{p}=0,008]$, hence the coiling group had a better post-procedure angiographic result. Concerning the risk of new intracranial hemorrhage events, we documented a statistically significant difference with higher risk in the stenting group [56,5\% in stenting group versus $7 \%$ in coiling group; $\mathrm{p}<0,001]$. There wasn't statistical difference in vasospasm $[33,8 \%$ in coiling group versus $39,1 \%$ in stenting group; $\mathrm{p}=0,411$ ] or refractory hydrocephalus risk [23,9\% in coiling group versus $43,5 \%$ in stenting group; $p$ value $=0,072]$. The stenting group presented a higher in-hospital mortality [8\% in coiling group versus $39,1 \%$ in stenting group; $p$ value $=0,05]$.

Conclusion: With this study we suggest that there is a higher hemorrhagic risk and in-hospital mortality in patients treated with intra-arterial stenting (with necessary dual antiplatelet therapy). The autor think that the use of stents in acute setting of aneurysmal rupture should be reviewed as a first line endovascular therapy.

\section{2-P8}

WASP TECHNIQUE IN M2 OCCLUSIONS IN THROMBECTOMYAN INITIAL EXPERIENCE

AIDAN HEGARTY ${ }^{1}$, Matthew Crockett ${ }^{1}$, Sarah Power $^{1}$, John Thornton $^{1}$, Paul Brennan ${ }^{1}$, Alan O'Hare ${ }^{1}$

${ }^{1}$ Beaumont Hospital, Beaumont Road, Dublin, Ireland

Keywords: Stroke, Thrombectomy, Aspiration

Introduction: Aspiration is a successful technique used in thrombectomy. At our institution we have previously demonstrated the efficacy of the Wedge microcatheter (Microvention) in allowing for the delivery of our aspiration catheter of choice (Sofia Plus) to an M1 occlusion when encountering challenging craniocervical vasculature. Following on from this we sought to assess how the Wedge and Sofia plus (WASP) combination would fare in the setting of M2 occlusions.

Methods: Our study cohort consisted of anterior circulation thrombectomies performed by five consultant neurointerventionalists from September 2020 to May 2021 inclusive. Cases in which the Wedge and Sofia plus catheters were used in the setting of an M2 occlusion were identified. Patient demographics, procedural details and outcomes were noted. The role of the Wedge catheter and whether it was successful in delivering the SofiaPlus catheter to the desired location was determined.

Results: The wedge microcatheter was used a total of twentyone times as the principal method of navigating the Sofia plus catheter to the site of distal occlusion. In twenty patients this was successful. Subsequent aspiration and extraction of $\mathrm{m} 2$ thrombus was achieved in all twenty of these patients with a first pass recanalization rate of $70 \%$. The remainder of patients required more than one pass to retrieve thrombus but scored a mTICI $2 b$ on final angiography. In only one patient, the occlusion could not be successfully accessed using the WASP technique thus necessitating clot retreival with a stent.

Conclusion: An aspiration first approach can be successfully employed in the setting of acute $\mathrm{m} 2$ occlusions in stroke. Initial experience at our institution demonstrates that using the WASP technique, access to occluded $\mathrm{m} 2$ branches and successful recanalisation can be achieved in the majority of cases. This technique offers a fast, safe and direct approach and adds further to our current technical skill set in acute stroke intervention. 
LONG-TERM OUTCOMES OF WIDE-NECKED INTRACRANIAL BIFURCATION ANEURYSMS TREATED WITH STENTASSISTED COILING USING LOW-PROFILE ACANDIS ACCLINO STENTS

DOMINIK GRIEB $^{1}$, Katharina Melber ${ }^{1}$, Frederik Boxberg ${ }^{1}$, Martin Schlunz-Hendann $^{1}$, Friedhelm Brassel ${ }^{2}$

${ }^{1}$ Department of Radiology and Neuroradiology, Sana Kliniken Duisburg, Duisburg, Germany, ${ }^{2}$ Sana Kliniken Duisburg, Duisburg, Germany

Keywords: Aneurysm, low-profile stents, long-term follow-up, stentassisted coiling

Background: Little data exists on endovascular treatment of complex intracranial aneurysms with the Acandis Acclino low-profile self-expanding closed-cell stent systems and is mainly limited to short- or midterm results. We report our long-term single center experience with the three generations of Acandis Acclino low-profile self-expanding closed-cell stent systems in the treatment of complex intracranial aneurysms.

Methods: 64 wide-necked intracranial aneurysms were treated electively using 91 Acandis Acclino stent systems. Single stent-assisted coiling was the preferred treatment in 40 cases and the kissing-Y stenting technique in 24 cases. We analyzed demographic data and long-term follow-up results.

Results: All stents were successfully deployed with immediate complete (RROC I) or near complete (RROC II) occlusion achieved in $92,2 \%$ of cases. Follow-up was available in 57 cases $(89,1 \%)$ with a mean followup of 37 months (range $6-80$ months). Long-term RROC I or II was achieved in 49 cases (86\%). Eight residual aneurysms (14\% RROC III) were noted ( 4 cases of stable residual aneurysmal filling and 4 of aneurysmal recanalization). Two of those recurrent aneurysms were retreated by coilembolization. The overall directly procedural-related complication rate was $4,7 \%$, including one death. Seven cases of in-stent-stenosis $(12,3 \%$; morbidity $n=0)$ were detected on long-term follow-up with 6 of them when using the kissing-Y stenting technique.

Discussion: Our high long-term follow-up occlusion rate (86\%) and overall in-stent stenosis rate confirm the previous promising, but so far limited, short- and midterm results of the Acclino stent systems and are comparable to other low-profile stents. However, it is remarkable that all but one clinically silent in-stent stenosis occurred when using the kissingY stenting technique. This is possibly attributable to the stent configuration in this dual-stent assisted technique. Our data included long term follow-up of the latest Acclino stent generation also associated with low-complication and aneurysmal recurrence rates.

Conclusion: Endovascular treatment of various complex intracranial aneurysms using the Acandis Acclino stent systems is safe and efficient with high aneurysm occlusion rates combined with low complication rates at long-term follow-up. Overall, rates of in-stent-stenosis are low but seem to depend on the treatment technique (single stent-assisted coiling versus kissing-Y stenting with coiling).

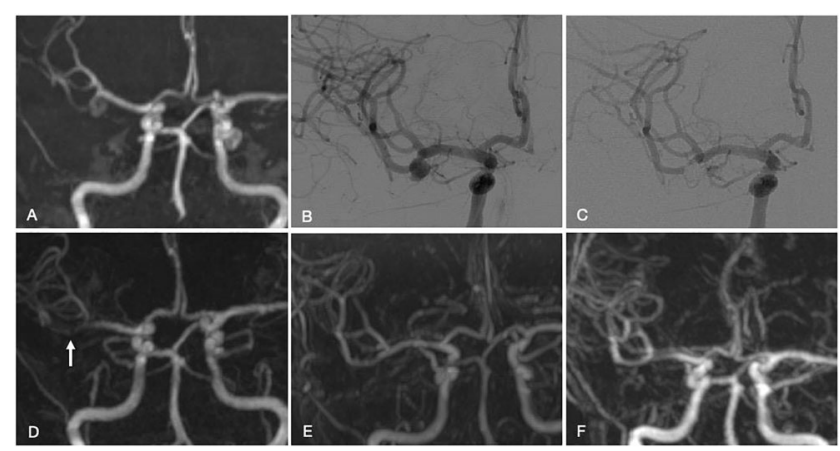

\section{HEAD \& NECK}

3-P1

OLFACTORY BULB MRI AND OLFACTORY TRACT DTI FINDINGS IN COVID-19 ANOSMIA AND OTHER POSTINFECTIOUS OLFACTORY DYSFUNCTION

$\underline{\text { SEDAT G. KANDEMIRLI }}{ }^{1}$, Duzgun Yildirim ${ }^{2}$, Deniz E. Tekcan Sanli ${ }^{3}$, Ozlem Saatci ${ }^{4}$, Aytug Altundag ${ }^{5}$

${ }^{1}$ University of Iowa Hospital and Clinics, Department of Radiology, Iowa, USA, ${ }^{2}$ Mehmet Ali Aydınlar University, Acıbadem Taksim Hospital, Department of Radiology, Istanbul, Turkey, ${ }^{3}$ Acibadem Kozyatağ Hospital, Department of Radiology, Istanbul, Turkey, ${ }^{4}$ Sancaktepe Training and Research Hospital, Department of Otolaryngology, Istanbul, Turkey, ${ }^{5}$ Biruni University, Medical Faculty; Acibadem Taksim Hospital, Department of Otolaryngology, Istanbul, Turkey

Keywords: olfactory bulb, COVID-19 anosmia, DTI

Introduction: We assessed the olfactory bulb MRI in persistent COVID19 anosmia; and compared the findings to other post-infectious OD. Our aim was to evaluate how COVID-19 anosmia imaging findings resembled and differed from post-infectious olfactory dysfunction, which would provide further insight into pathogenesis of COVID-19 anosmia.

Materials \& Methods: 31 patients presenting with persistent OD after confirmed COVID-19 infection, and 97 patients presenting with olfactory dysfunction after upper respiratory tract infection were included. Evaluation included detailed olfactory anamnesis and otolaryngological examination. Olfactory function was evaluated with Sniffin' Stick Test. Subsequently, olfactory bulb MRI and DTI were performed. Olfactory bulb (OB) volumes were calculated, and OBs were assessed for shape and signal abnormalities. Olfactory nerve filiae were evaluated for clumping. Quantitative anisotropy (QA) values of orbitofrontal and entorhinal regions were measured.

Results: Mean interval between onset of OD and imaging was 1.5 months for COVID-19 related OD and 6 months for post-infectious OD. Mean TDI score was significantly lower in COVID-19 related OD $(9,3)$ than post-infectious OD $(12,7)$.

OB volumes were significantly lower in post-infectious OD (R: 48,6 $\mathrm{mm} 3$; L: 48,9 mm3) than COVID-19 related OD (R: $60,3 \mathrm{~mm} 3$; L: $59,5 \mathrm{~mm} 3$ ) Deformed bulb morphology was seen in 62 cases $(63.9 \%)$ with post-infectious OD and 18 cases (58.1\%) with COVID-19 related OD; without significant difference between the groups. Abnormal bulb signal intensity was seen in 45 patients $(46.4 \%)$ with post-infectious OD and 16 patients $(51.6 \%)$ with COVID-19 related OD without significant difference between the groups. 
There was a significantly higher rate of olfactory nerve clumping in COVID-19 related OD (71\%) than post-infectious OD (29,9\%). QA values at orbitofrontal and entorhinal regions were significantly higher in COVID-19 related OD than post-infectious OD

Conclusion: Our study suggests that olfactory bulb damage as demonstrated by abnormal shape and signal intensity plays a role in COVID-19 anosmia. Though there is decreased olfactory bulb volume in COVID-19 anosmia, this is not as pronounced in other post-infectious OD, likely reflecting earlier imaging done in COVID-19 cases with limited duration of olfactory deprivation to result in olfactory bulb volume loss. We observed a higher rate of olfactory nerve clumping in COVID-19 anosmia compared to postinfectious OD, indicating a higher degree of regeneration attempt. The white matter tract integrity of olfactory tracts were better preserved in COVID-19 anosmia compared to post-infectious OD, which also might be related to shorter imaging duration since onset of olfactory loss.

\section{3-P2}

COMPARISON OF DW-MRI AND 18F-FDG PET/CT IN DETECTION OF RESIDUAL/RECURRENT TUMORS AND DELINEATION OF THEIR LOCAL SPREAD AFTER (CHEMO) RADIOTHERAPY FOR HNSCC COMPARED WITH THE GOLD STANDARD OF HISTOPATHOLOGY

\section{CHRISTOPHE SCHROEDER $^{1}$, Jung-Hyun Lee ${ }^{2}$, Soung Yung Kim ${ }^{3}$}

${ }^{1}$ University Institute for Diagnostic, Interventional and Paediatric Radiology, Inselspital University Hospital Bern, University of Bern, Freiburgstrasse 10, 3010 Bern, Switzerland, ${ }^{2}$ Institute of Molecular Virology, Ulm University, Ulm, Germany, ${ }^{3}$ Department of Diagnostic and Interventional Radiology, University Hospital Ulm, Ulm, Germany

Keywords: Head and neck squamous cell carcinoma, Radiology, Nuclear medicine

Introduction: Detection of residual or recurrent tumors in patients treated for head and neck squamous cell carcinoma (HNSCC) can be very challenging. This retrospective study compares the diagnostc accuracy of diffusion-weighted MR imaging (DW-MRI) and fluorine $18 \mathrm{~F}$ fluorodeoxyglucose (18F-FDG) PET/CT in detection residual or recurrent tumors and their local extension, in patients with $\mathrm{HNSCC}$ after treatment with (chemo) radiotherapy (CRT).

Methods: Twenty-five patients ( 17 men, 8 women, median age 64 years, range 53-86) who underwent surgical salvage for residual or recurrent tumors after CRT were included. The DW-MRI and PET/CT images were anonymized and reviewed retrospectively. The results were compared to the histopathology reports.

Results: Both DW-MRI and18F-FDG PET/CT had a sensitivity of $92 \%$ $(23 / 25)$ in the detection of residual or recurrent tumors. MRI had a sensitivity, specificity, positive predictive value (PPV) and negative predictive value (NPV) for detecting perineural spread of $62 \%(5 / 8), 88 \%(15 / 17)$, $71 \%(5 / 7)$ and $83 \%(15 / 18)$, respectively; in comparison, PET/CT did not detect any cases of perineural spread. The sensitivity, specificity, PPV and NPV of MRI in detecting muscle infiltration was $75 \%(9 / 12), 77 \%(10 /$ $13), 75 \%(9 / 12)$ and $77 \%(10 / 13)$ respectively, while the values for $18 \mathrm{~F}$ FDG PET/CT were $58 \%(7 / 12), 69 \%(9 / 13), 64 \%(7 / 11)$ and $64 \%$ $(9 / 14)$.

Conclusions: DW-MRI and 18F-FDG PET/CT have an identical detection rate of residual or recurrent tumors after CRT. MRI has a higher sensitivity in detecting local perineural spread, has a better accuracy in the detection of muscle infiltration and more accurately correlates the lesion size to the histo-pathologic specimen.
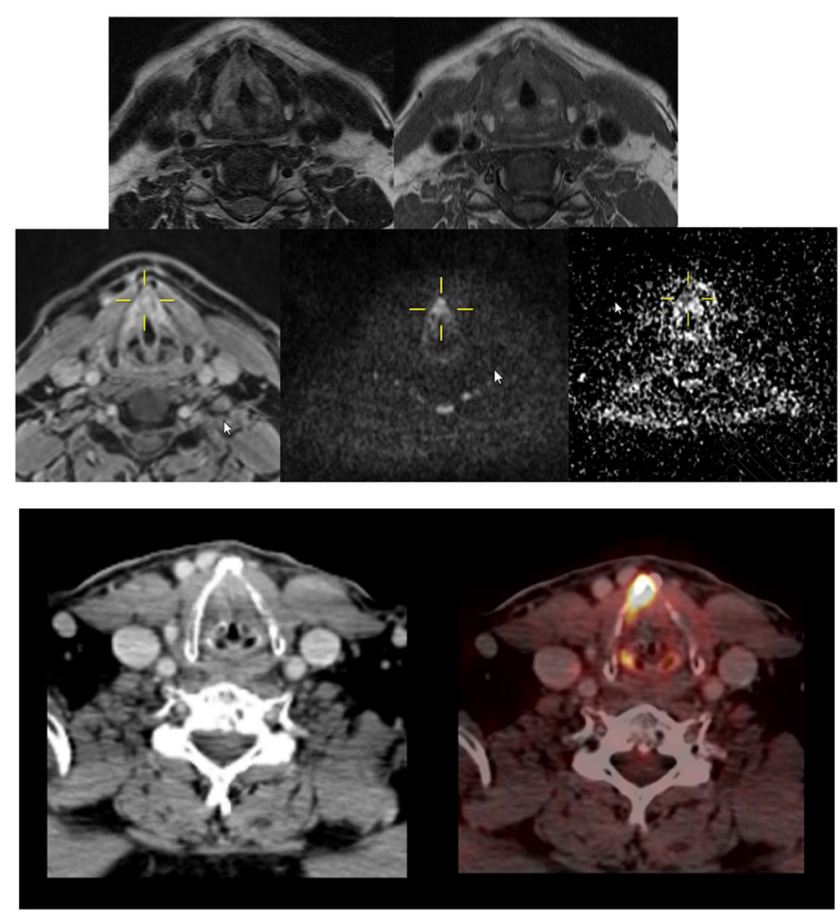

3-P3

UNILATERAL SINONASAL LESIONS: INVERTED PAPILLOMA AND BEYOND

FATIMA HIERRO $^{1}$, Pedro Miguel Marques Gomes ${ }^{2}$, Paula Azevedo ${ }^{2}$, Rute Garcia

${ }^{1}$ Neuroradiology Department, Hospital Pedro Hispano, Matosinhos, Portugal, ${ }^{2}$ Otolaryngology Department, Hospital Pedro Hispano, Matosinhos, Portugal

Keywords: nasal, Unilateral, Tumor, hemangioma, papilloma, melanoma, sarcoma, lymphoma, meningoencephalocele

Introduction: Persistent unilateral nasal obstruction is strongly suggestive of a structural anomaly and a neoplastic cause should always be considered. A thorough clinical history and physical examination, together with imaging, are essential in outlining possible causes. The visualization of a growth on nasosinusal endoscopy should prompt a CT exam. Due to these lesions' easy access, an early biopsy can be considered. Here we address six types of nasal tumors that presented with persistent unilateral nasal obstruction: lobular capillary hemangioma, inverted papilloma, melanoma, diffuse large cell B lymphoma, myeloid sarcoma and meningoencephalocele.

Methods: Descriptive observational study of six patients presenting with persistent unilateral nasal obstruction during 2 consecutive years at ENT consultation with unusual unilateral nasal tumors were selected.

Results: The patients age range from 20 to 87 years (mean: 58 years), $66 \%$ female. The youngest patient had a lobular capillary hemangioma and the older had diffuse large B cell lymphoma. All the patients underwent CT. MRI was performed in the investigation of patients with myeloid sarcoma and meningoencephalocele. The hemangioma 
appearance on CT was of a mass in the right nasal cavity, without associated bone erosion (image 1). The inverted papilloma filled the right maxillary sinus, extending through the maxillary antrum to the nasal fossa, with bone remodeling (image 2). The melanoma completely filled the right nasal cavity, extending to the nasopharynx, without associated bone erosion (image 3). Diffuse large B cell lymphoma presented as a left nasal mass that caused erosion of the adjacent bone (image 4). Myeloid sarcoma was a large nasal mass, extending to the right frontal, maxillary and ethmoidal sinuses, eroding the adjacent bone structures (image 5a). MRI showed bone and muscle infiltration of the orbit (image 5b). Meningoencephalocele caused bone remodeling, with dehiscence of the skull base (image 6). An histological diagnosis was obtain after the imaging study.

Discussion: In accordance with literature, bone erosion and soft tissue invasion were associated with malignant neoplasms. Bone remodeling, the presence of well-defined borders and the absence of invasion of adjacent tissues, point to a more benign behaviour.

Conclusion: Unilateral nasal tumors are a rare cause of nasal obstruction. A high index of suspicion should be maintained if nasal obstruction is unilateral and persistent. In this series, we illustrate imaging features that can help in the differential diagnosis of benign, malignant and cerebral herniation tissue. Imaging combined with clinical presentation are essential when considering an immediate biopsy.
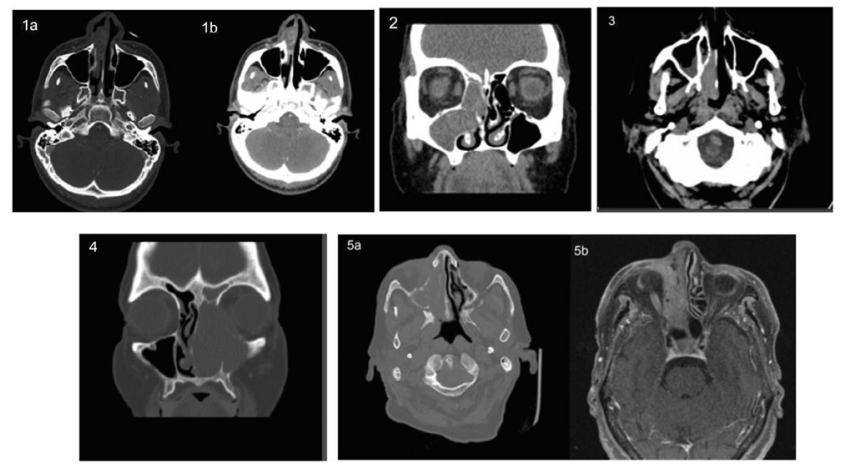

\section{3-P4}

OCULAR MRI FINDINGS IN PATIENTS WITH SEVERE COVID19: A RETROSPECTIVE MULTICENTER OBSERVATIONAL STUDY

AUGUSTIN LECLER $^{1}$, Francois Cotton ${ }^{2}$, Francois Lersy ${ }^{3}$, Stephane Kremer $^{3}$, Francoise Heran ${ }^{1}$ on behalf of the SFNR's COVID study group

${ }^{1}$ Department of Neuroradiology, Rothschild Foundation Hospital, Paris, France, ${ }^{2}$ Department of Neuroradiology, CHU Lyon, Lyon, France, ${ }^{3}$ Department of Neuroradiology, CHU Strasbourg, Strasbourg, France

Keywords: COVID-19, Orbit, MRI

Background: COVID-19 may affect various organs. Ophthalmological abnormalities are relatively rare among COVID-19-infected patients.

Purpose: To report ophthalmological MRI findings in a nationwide multicenter cohort of patients with severe COVID-19.

Materials \& Methods: This IRB-approved retrospective multi-center study included participants presenting with severe COVID-19, who underwent brain MRI from March 4th to May 1st 2020. Two neuroradiologists blinded to all data, individually analyzed morphological MRIs focusing on the eyeballs, the orbits and the visual pathways. A second consensus reading session was performed in case of disagreement between both readers. Clinical and ophthalmological data were compared to MRI findings. Descriptive statistical analysis and interobserver agreement for MRI reading using non-weighted Cohen kappa statistics were performed.

Results: 129 participants (43 [33\%] women and 86 [67\%] men, mean age $63.3 \pm 13.8$ years) were included in the study. $30 / 129(23 \%)$ patients had abnormal MRI findings of the eyeball, orbit or visual pathways. 9/30 (30\%) patients had one or several FLAIR-WI hyperintense nodules of the posterior pole of the eyeball. Nodules were bilateral in $8 / 9(89 \%)$ patients, located in the macular region in all cases and outside the macular region in 2/9 (22\%) patients. $3 / 30(10 \%)$ patients had thickening of the external ocular layer. 10/30 (33\%) patients had a FLAIR-WI hyperintense optic disc. $6 / 30(20 \%)$ patients had abnormal signal of at least one of the visual pathway structures: $6 / 6(100 \%)$ of the optic nerve, $1 / 6(17 \%)$ of the optic chiasm, $2 / 6(33 \%)$ of the optic tract and 1/6 (17\%) of the optic radiations.

Conclusion: Our study showed that a substantial number of patients with severe COVID-19 presented with abnormal MRI findings of the eyeball, orbit or visual pathways. Screening of these patients might be suitable to provide appropriate treatment and improve the management of potentially severe ophthalmological manifestations.
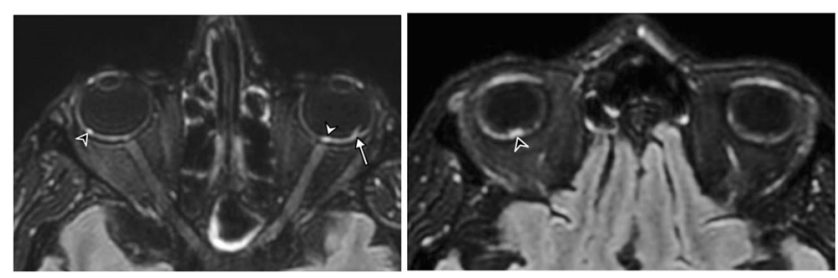

3-P5

DIXON-T2WI MAGNETIC RESONANCE IMAGING AT 3 TESLA OUTPERFORMS CONVENTIONAL IMAGING FOR THYROID EYE DISEASE

ALEXIS OLLITRAULT $^{1}$, Frederique Charbonneau ${ }^{1}$, Olivier Berges ${ }^{1}$, Aviva Herdan ${ }^{2}$, Kevin Zuber ${ }^{3}$, Lama Giovansili ${ }^{4}$, Julien Savatovsky ${ }^{1}$, Augustin Lecler ${ }^{1}$

${ }^{1}$ Department of Neuroradiology, Foundation Adolphe de Rothschild Hospital, Paris, France, ${ }^{2}$ Department of Orbitopalpebral Surgery, Foundation Adolphe de Rothschild Hospital, Paris, France, Department of Clinical Research, Foundation Adolphe de Rothschild Hospital, Paris, France, ${ }^{4}$ Department of Internal Medicine, Foundation Adolphe de Rothschild Hospital, Paris, France

Keywords: Magnetic resonance imaging, Eye diseases, Thyroid disease, Inflammation, Artifacts

Objectives: To determine the diagnostic performances of a single DixonT2-weighted imaging (WI) sequence compared to a conventional protocol including T1-, T2-, and fat-suppressed T2-weighted MRI at 3 T when assessing thyroid eye disease (TED).

Materials \& Methods: This IRB-approved prospective single-center study enrolled participants presenting with confirmed TED from April 2015 to October 2019. They underwent an MRI, including a conventional protocol and a Dixon-T2WI sequence. Two neuroradiologists, blinded to 
all data, read both datasets independently and randomly. They assessed the presence of extraocular muscle (EOM) inflammation, enlargement, fatty degeneration, or fibrosis as well as the presence of artifacts. The Wilcoxon signed-rank test was used.

Results: Two hundred six participants were enrolled (135/206 [66\%] women, 71/206 [34\%] men, age $52.3 \pm 13.2$ years). Dixon-T2WI was significantly more likely to detect at least one inflamed EOM as compared to the conventional set (248/412 [60\%] versus 228/412 [55\%] eyes; $(p=$ 0.02 ). Dixon-T2WI was more sensitive and specific than the conventional set for assessing muscular inflammation (100\% versus $94.7 \%$ and $71.2 \%$ versus $68.5 \%$, respectively). Dixon-T2WI was significantly less likely to show major or minor artifacts as compared to fat-suppressed T2WI (20/ 412 [5\%] versus 109/412 [27\%] eyes, $\mathrm{p}<0.001$, and 175/412 [42\%] versus $257 / 412[62 \%]$ eyes, $\mathrm{p}<0.001$ ). Confidence was significantly higher with Dixon-T2WI than with the conventional set (2.35 versus $2.24, \mathrm{p}=0.003)$

Conclusion: Dixon-T2WI showed higher sensitivity and specificity and showed fewer artifacts than a conventional protocol when assessing thyroid eye disease, in addition to higher self-reported confidence.

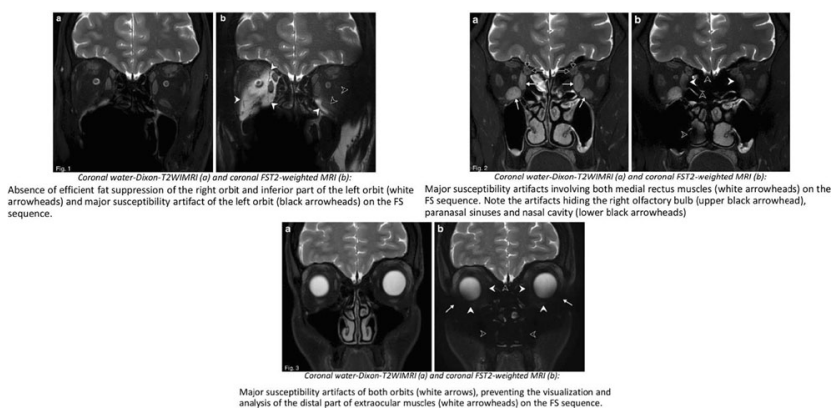

\section{3-P6 \\ VALUE OF DELAYED ENHANCED 3D FLAIR MRI IN PATIENTS WITH ACUTE VESTIBULAR SYNDROME}

\section{$\underline{\text { DONG WOO PARK }}$}

${ }^{1}$ Hanyang University Guri Hospital, Guri, Korea

Keywords: acute vestibular syndrome, delayed enhanced 3D FLAIR MRI, enhancement of affected labyrinth

Purpose: This study aims to evaluate the affected labyrinth including vestibule and semicircular canals on delayed post-contrast 3D FLAIR MRI in patients with unilateral acute vestibular syndrome (UAVS).

Methods: 118 patients who presented with UVAS and underwent labyrinthine MR imaging, including 3D FLAIR MR images before and 4 hour after Gadolinium injection, were retrospectively analyzed, focusing on asymmetric affected labyrinthine enhancement. Vestibular function tests including the caloric and video head impulse tests were performed. Interreader agreement tests were performed.

Results: A delayed enhancement of the affected vestibule and/or semicircular canals on 3D FLAIR MRI was observed in 97 out of 118 ears $(82.2 \%, \mathrm{p}<0.001)$, but delayed enhancement of the cochlea was observed in 42 out of 118 ears (35.6\%).
The duration of spontaneous nystagmus was significantly longer in the patients with enhancement, especially the whole labyrinth including the vestibule and semicircular canals $(\mathrm{P}<0.05)$.

Conclusion: The enhancement of affected labyrinth of vestibule and/or semicircular canal on delayed post-contrast 3D FLAIR MRI was presented in patients with UAVS. The degree of its enhancement may be associated with the duration of spontaneous nystagmus. The delayed affected labyrinthine enhancement in patients with UAVS could be the MR imaging biomarker for the initial and prognostic values for UAVS.

3-P7

MR IMAGING SPECTRUM IN ACUTE INVASIVE FUNGAL RHINOSINUSITIS FOLLOWING COVID-19 INFECTION TREATED WITH STEROIDS

KANIKA SEKHRI SETHI ${ }^{1}$, Anmol Uberoi ${ }^{1}$, W V B S Ramalingam ${ }^{2}$, Neha Sood $^{2}$, Prem Kumar Ganesan ${ }^{1}$, Payal Mital ${ }^{1}$

${ }^{1}$ Department of Radiology, BLK Max Superspeciality hospital, New Delhi, India, ${ }^{2}$ Department of E.N.T, BLK Superspeciality hospital, New Delhi, India

Keywords: Acute invasive fungal rhinosinusiti, COVID-19, hyperglycemia

Introduction: Acute invasive fungal rhinosinusitis (AIFRS) is an acute and life threatening rapidly progressing infection caused by fungi of class phycomycetes. COVID-19 infection may be associated with fungal coinfections, especially post-steroid treatment and having severely impaired glycemic control. MRI plays an important role in early diagnosis and extent of this fulminant infection. The objective of this study is to describe the spectrum of MR imaging findings.

Methods: This is a prospective study done during the second wave of SARS-Coronavirus 2 COVID-19 infection in India from April to May,2021. Statistical analysis was done using descriptive statistics. A total of 38 patients presented with confirmed AIFRS. Out of these 38, 32 patients with mean age 54 years who had recovered from COVID-19 illness, had received steroids, had hyperglycemia and later presented with nasal, orbital and cranial symptoms to our hospital were included in this study and later confirmed by biopsy or microbiology (KOH smearmounts). 6 patients were excluded from the study due to absence of history of COVID-19 illness, immunocompromised status.

MRI of the PNS and orbits were performed on a $1.5 \mathrm{~T}$ system (GE Signa Artist TM) using T1, T2W and STIR coronal \& axial sequences, SWI, 3D FIESTA/CUBE and T2W axial \& DWI sequences of brain and followed by contrast enhanced T1W FS sequences through the PNS, orbits and brain after IV injection of Gadopentate dimeglumine $(0.1 \mathrm{mmol} / \mathrm{kg})$. 3D TOF MR angiography was acquired whenever there was evidence of angioinvasion. Images were evaluated for signal intensity, contrast enhancement characteristics and extent.

Results: MRI of 32 patients showed predominant involvement of the paranasal sinuses (32 patients, $100 \%$ ), nasal cavity (18 patients, $56 \%$ ), orbits (15 patients, $47 \%$ ), soft tissue including premaxillary, retroantral \& pterygopalatine fossa (18 patients, 56\%), involvement of skull base (11 patients, $34 \%$ ) and finally intracranial spread (8 patients, 25\%). Nonenhancing turbinates (black-turbinate sign) was seen in 15 cases (47\%). Soft tissue thickening showed heterogeneous hypointense signal on T2W and heterogenous peripheral enhancement with central non-enhancing areas as the main finding in 15 patients $(47 \%)$. Among intracranial spread, 6 patients had cavernous sinus thrombosis (19\%) and 4 patients 
developed infarcts due to vascular involvement (12.5\%). Perineural spread was seen in one patient (3\%).

Conclusion: Contrast enhanced MRI shows a diverse spectrum of findings in AIFRS which plays a crucial role in assessing the extent of involvement, guiding treatment protocols and predicting the complications.

\section{3-P8}

ASSESSMENT OF ANATOMIC VARIATIONS AND MEASUREMENTS IN HIGH RESOLUTION COMPUTED TOMOGRAPHY RELATED TO ROUND WINDOW VISIBILITY IN COCHLEAR IMPLANTATION

EDUARDA MARIA MARINHO PINTO ${ }^{1}$, Catarina Mendes Pinto ${ }^{1}$, Joao Lino ${ }^{2}$, Joao Almeida ${ }^{2}$, Bruno Moreira

${ }^{1}$ Centro Hospitalar Universitario do Porto, Department of Neuroradiology, Porto, Portugal, ${ }^{2}$ Centro Hospitalar Universitario do Porto, Department of Otorhinolaryngology, Porto, Portugal

Keywords: cochlear implantation, round window visibility, HRCT prediction

Introduction: During cochlear implantation, clear visualization of the round window niche (RWN) via posterior tympanotomy is important for the surgeon to properly insert the electrode in the scala tympani. High resolution computed tomography (HRCT) assessment of the position of the facial nerve (FN), measurements involving anatomical landmarks and evaluation of morphological variability of the RWN were previously associated with the grade of RWN and membrane visibility. We aimed to access the $\mathrm{CT}$ anatomic variations and measurements in our cases that could help predict poor RWN visibility.

Methods: We retrospectively reviewed the presurgical CT of 45 cochlear implant ears. We differentiated RW shape in cylindrical/cone types or " $\mathrm{J}$ "type and measured two parameters: the angle between the coronal axis, the $\mathrm{RWN}$ and a line anterior to $\mathrm{FN}$, and the angle between the posterior wall of external auditory canal (EAC), the RWN, and a line anterior to FN; if the sigmoid sinus was anterior to this line, the line was drawn tangential to its limit. The surgical notes were reviewed to access RWN visibility.

Results: Of 45 cases, 5 had poor intraoperative visibility of RWN, 2 of which were invisible. Three of these five cases had the smallest angle of the posterior wall of EAC (9\%-15\%). The other two had a "J"-type RWN, that was also hypoplastic. Regarding the cases with invisible RWN, one had an anteriorized mastoid segment of facial nerve, with the smallest angle of the coronal axis $\left(11^{\circ}\right)$, and the other had a very acute angle of the posterior wall of EAC (10\%).

Discussion: A previous study related radiological morphology of RWN with endoscopic anatomy and associated "J"-type with difficult visibility of RW. We had difficulty with classification of one case with good visibility that was considered a "J"-type; cases with a clear "J"-type had poor visibility of RWN, but they were also hypoplastic. In our cases, the angle of the posterior wall of the EAC was more consistent in differentiating the cases with poor and good RWN visibility, than the angle of the coronal axis. Nevertheless, an anterior course of the facial nerve is reported to restrict RWN visibility.

Conclusion: We believe many factors influence the visibility of RWN, namely RWN morphology and variations of anatomical surgical landmarks. The angle of the posterior wall of EAC was a good measurement to evaluate RWN visibility in our cases, but further studies with more cases are needed to apply it.

3-P9

ORBITAL LYMPHOMA: 15 YEARS UNTIL DIAGNOSIS. CASE REPORT OF AN EXTRA-OCULAR ORBITAL NON-HODGKIN LYMPHOMA

FRANCISCA SENA BATISTA $^{1}$, Filipa Maria Neves Castelao ${ }^{1}$, Diogo Andre Gomes Ferreira Marques ${ }^{1}$, Mariana Cardoso Diogo ${ }^{1}$, Ligia Neves $^{1}$, Ana Cristina Rodrigues Rios ${ }^{1}$

${ }^{1}$ Neuroradiology Department, Hospital Garcia de Orta, Almada, Portugal

Keywords: orbit, lymphoma, exophthalmos

Introduction: Lymphomas are part of the differential diagnosis of orbital masses, but their diagnosis is not always easy and can often be protracted. We present a case of a type B non-Hodgkin lymphoma presenting with a story of 15 years of exophthalmos. We further present imaging characteristics and pearls and pitfalls of the differential diagnosis of lacrimal gland region.

Methods: Case report with detailed clinical, computed tomography and magnetic resonance imaging, as well as histologic data will be presented, together with cases of different pathologies that may mimic clinically and imagiologically this entity. Clues to diagnosis will be presented in a didactic manner.

Results: Case report: A 47 year old woman presented with left eye exophtamos, with 15 years evolution. CT and RMN Orbit were performed, revealing an extra-ocular lacrimal gland region mass, indistinguishable of the lacrimal gland with low SI on T1WI, high SI on T2WI and DWI and homogeneous enhancement. Biopsy of the mass revealed extranodal marginal zone B-cell lymphoma of MALT type (CD20+, bcl2+, CD3-, CD5-, CD23-, CD10-, bcl6-, cyclin D1-). Ocular adnexal lymphomas can be primary or secondary, and may be solitary or multiple, localised or extensive. Imaging characteristics alone do not allow specific diagnosis but can help differentiate between being and malignant lesions, and help in the decision to intervene or "leave-it-alone". Being aware of these entities is essential as is recognising main differential diagnosis. We present pearls and pitfalls of differential diagnosis of lacrimal gland region masses, including: lymphoma, rhabdomyosarcoma, lacrimal gland tumors, other head and neck tumours, orbital metastasis (including systemic lymphoma), idiopathic orbital inflammation or Ig-G4 related orbital disease and benign and malignant bone lesions.

Imaging evolution is also presented.

Conclusion: Lymphomas are often difficult to diagnose with many different entities presenting with clinical and imagiological overlapping features. We present one case with symptoms going back 15 -years from time of diagnosis, emphasising the wide range of clinical presentations. Knowledge of the imaging features of these diverse entities is essential for radiologists in training and specialists alike.

\section{PEDIATRIC}

\section{4-P1}

THE SPECTRUM OF NEUROIMAGING FINDINGS IN STURGEWEBER SYNDROME: AN EDUCATIONAL PICTORIAL REVIEW

OLGA MEDVEDEV $^{1}$, Otilia Fufezan ${ }^{2}$, Carmen Asavoaie $^{2}$ 
${ }^{1}$ Diagnostic Radiology, County Emergency Hospital of Cluj-Napoca, Romania, ${ }^{2}$ Pediatric Clinical Emergency Hospital, Cluj-Napoca, Romania

Keywords: Sturge-Weber Syndrome, port-wine stain, leptomenigeal angiomas, brain atrophy, cortical calcifications, MRI with gadolinium

\section{Learning Objectives:}

- to review the literature regarding the Sturge-Weber syndrome (SWS) pathology and how it relates to the morphologic changes and the corresponding symptoms;

- to illustrate the key neuroimaging findings in a pictorial educational review of cases from the institution database;

- to increase the awareness of the need to timely diagnose the SWS.

Background: SWS is a phakomatosis characterized by leptomeningeal angiomas (LAs), glaucoma and the hallmark which is the facial port-wine stain (PWS) typically in the ophthalmic or maxillary branches of the trigeminal nerve. The somatic activating mutations in the GNAQ gene constitute the cause; however there are still limitations in relating the symptoms to the underlying pathophysiology. We briefly illustrate the current information in pathogenesis with the resulting clinical manifestations, especially relating to the brain involvement with the corresponding major neuroimaging findings. The clinical phenotype ranges from uncomplicated PWS to complex neurocutaneous and ophthalmologic manifestations. The neurological symptoms such as seizures, hemiparesis, stroke-like episodes, visual field deficits, delayed development appear early in infancy and progress in various degrees, but sometimes may first present in adulthood.

Imaging Findings: Being a rare and complex syndrome, it is not always an easy diagnosis both clinically and radiologically. We aimed to review the current state of knowledge in the neuroimaging of SWS. MRI is the key imaging modality for diagnosis of SWS, as it provides crucial information about the presence, extent and severity of brain parenchymal changes (figure 1-2). The classical finding is the leptomeningeal enhancement on post-contrast sequences meaning the LAs. Some cerebral lesions can be detected in the neonatal phase, others only after the progression of the disease, because they result from the hemodynamic disturbances. Seizure control, aspirin and early surgery may prevent neurological deterioration and improve the quality of life; therefore an earlier diagnosis aided by imaging is vital. According to the available literature standardized neuroimaging studies at the specific time points in the disease course are needed (table 1). Sonography and CT may also be used, but have a limited role. A summary of the diagnostic value of each neuroimaging technique together with the specific imaging findings is depicted in table 2 .

Conclusion: SWS disease progression is still unclear and therefore prospective studies with integrated clinical, imaging and neuropahtological information are needed. There is a necessity for clear guidelines on care and follow-up, among which imaging plays an important role in brain involvement detection.
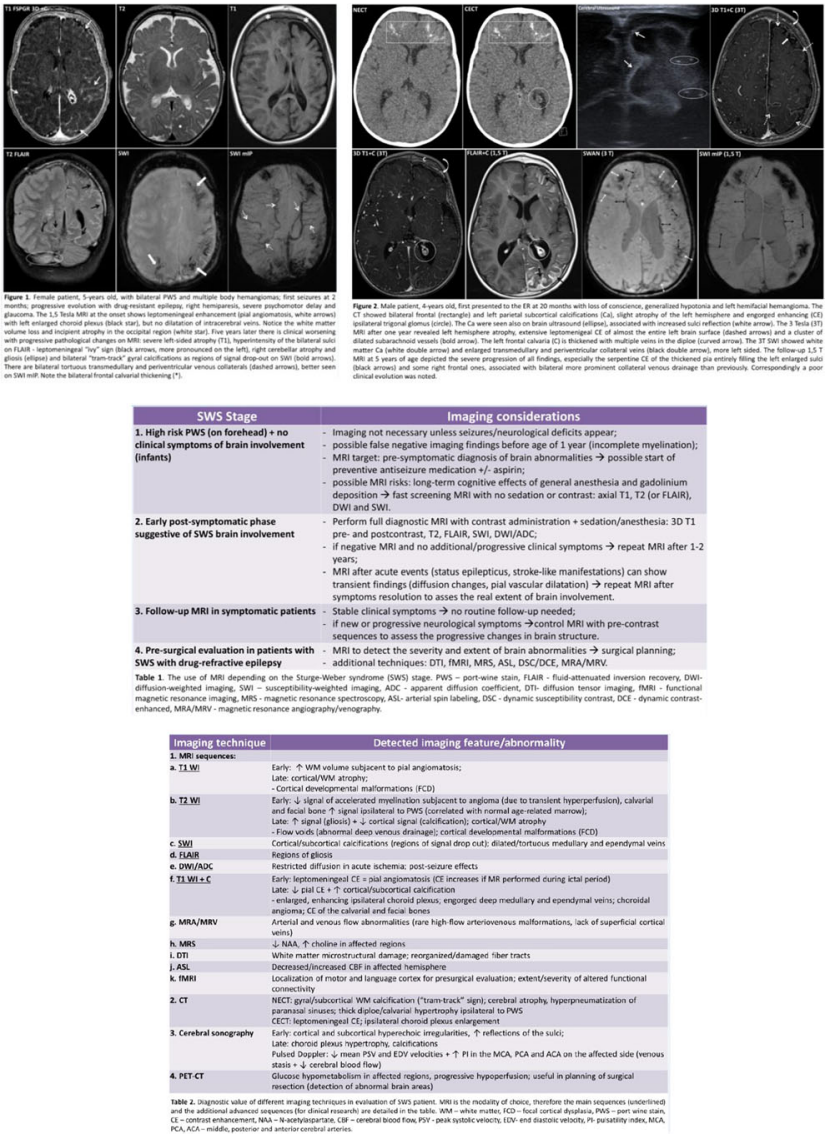
4-P2

ENCEPHALIC CHANGES BY FETAL MAGNETIC RESONANCE IN FETUSES WITH ISOLATED CONGENITAL HEART DISEASE

\section{LINA FERNANDA GAMBOA LATORRE ${ }^{1}$, Luisa Fernanda Jaimes Martinez ${ }^{2}$, Saulo Molina Giraldo}

${ }^{1}$ Radiology Department, Fundacion Universitaria de Ciencias de la Salud, Bogota, Colombia, ${ }^{2}$ Radiology Department, Hospital San Jose, Bogota, Colombia, ${ }^{3}$ Maternal and Fetal Medicine, Hospital San Jose, Bogota, Colombia

Keywords: neurodevelopment, fetal magnetic resonance imaging, Congenital heart disease

The Department of Radiology of the Hospital San Jose Infantil (University Hospital attached to the Fundacion Universitaria de Ciencias de la Salud in Bogota, Colombia), carried out a characterization of brain lesions by fetal magnetic resonance in patients with a diagnosis of cyanotic and non-isolated congenital fetal heart disease. cyanose. Patients with an ultrasound diagnosis of Congenital Heart Disease were selected by the Maternal Fetal Medicine Service, subsequently a structured report was made focused on the characterization of lesions and other findings of the neuraxis by Fetal Magnetic Resonance. Given that there is still no evidence of the true relationship between congenital heart disease and fetal brain injuries in the prenatal period, this study seeks to generate new knowledge about this possible causal relationship in order to improve the approach, management, follow-up and advising these patients in an optimal and early way.

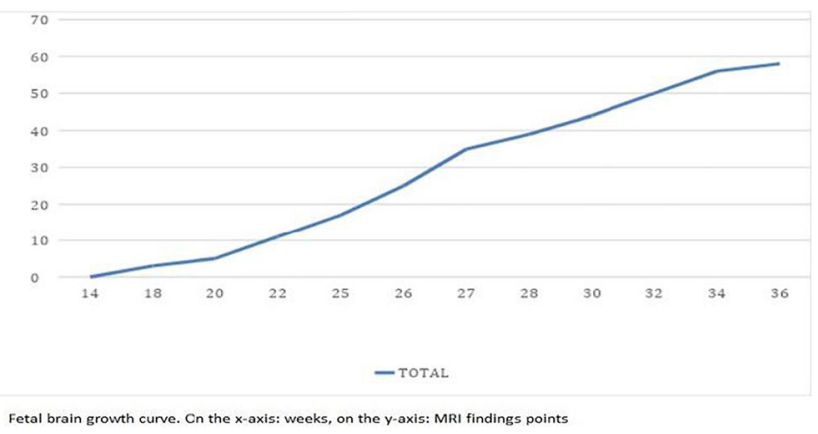

4-P3

PICTORIAL REVIEW OF MRI FINDINGS IN LEUKODYSTROPHY

ABHISHEKH ASHOK ${ }^{1,2}$, Francis Scott $^{1}$, Deepa Krishnakumar ${ }^{3}$, Pooja ${\text { Harijan }^{3} \text {, Chitre Manali }}^{3}$, Gautam Ambegaonkar ${ }^{3}$, Alasdair P. J. Parker ${ }^{3}$, Poe Phyu ${ }^{1}$, Justin Cross ${ }^{1}$

${ }^{1}$ Department of Radiology, Addenbrooke's Hospital, Cambridge University Hospital NHS Foundation Trust, Cambridge, UK, ${ }^{2}$ Department of Radiology, University of Cambridge, Cambridge, UK, ${ }^{3}$ Department of Paediatrics, Cambridge University Hospital NHS Foundation Trust, Cambridge, UK

Keywords: Paediatric, leukodystrophy, MRI

The leukodystrophies comprise a spectrum of disorders secondary to enzyme deficiencies that result in abnormal myelination. The most recent classification separates these into myelin disorders, astrocytopathies, leukoaxonopathies, microgliopathies and leukovasculopathies. The clinical manifestations of these disorders can vary, and MRI is central to the initial diagnostic workup. The approach to MRI interpretation should include identifying bilateral symmetric white matter involvement, any anatomical distribution pattern, presence of calcification and/or microhaemorrhage, the enhancement pattern and spinal cord involvement. This educational exhibit will include series of cases of genetically confirmed leukodystrophy.

The following are representative cases which will be described in the exhibit:

Case 1: POLR3-Related Leukodystrophy. A 3-year-old child presented with difficulty in walking and ataxia. MRI showed prominent cerebellar atrophy associated with bilateral FLAIR hyperintensity in the periventricular white matter and delayed myelination in the posterior limb of internal capsule. Subsequent genetic testing identified a mutation in POLR3B gene.

Case 2: X-linked Adrenoleukodystrophy. A 7-year-old male child presented with seizure, divergent squint and hearing difficulty. MRI showed bilateral symmetric T2 hyperintensity in the periventricular region with posterior predominance. Subsequent genetic testing revealed a mutation in $\mathrm{ABCD} 1$.

Case 3: Alexander's disease. A 2-year-old male child presented with seizure and developmental delay. MRI showed bilateral symmetric T2 hyperintensity in the periventricular region with frontal predominance. Subsequent genetic testing identified a mutation in the GFAP gene.

Case 4: Coats plus syndrome. A 3-year-old child presented with hepatic dysfunction, gastrointestinal bleed and global developmental delay. MRI showed focal areas of T2 hyperintensity in the pons, midbrain, thalamus, basal ganglia, and parietal white matter bilaterally. Subsequent genetic testing revealed Coats plus syndrome due to mutation in the STN1 gene.

Case 5: Metachromatic leukodystrophy. A 10-year-old child presented with toe walking and poor balance over a three-year period. MRI showed bilateral symmetric T2 hyperintensity in the periventricular region with posterior predominance. Subsequent genetic testing revealed mutation in ARSA gene.

Conclusion: A wide variety of leukodystrophies can present with similar clinical features. MRI is critical in narrowing the differential diagnosis and assessment of disease progression. Availability of genetic testing and inclusion of these disorders in neonatal screening is leading to rapid advances in the field. Prompt radiological recognition is critical in enabling early initiation of treatment and genetic counselling of family. Recognising these disorders is also important in the context of the rapid advances in regenerative approaches which may translate to clinical practice in the next decade. 

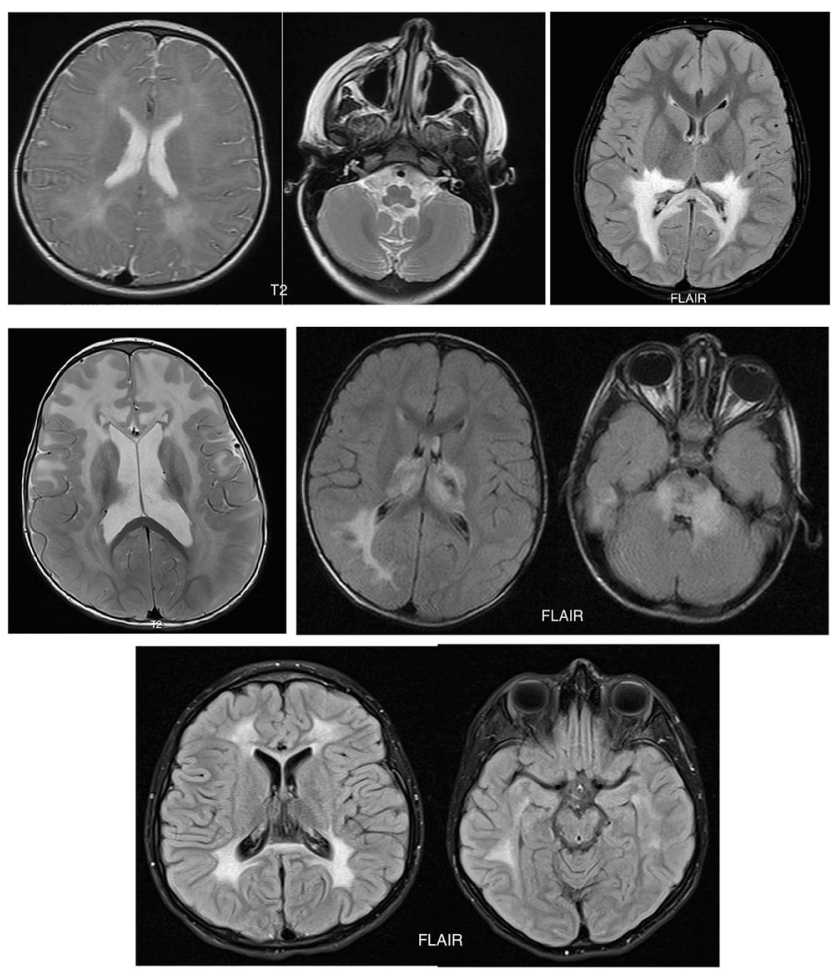

\section{4-P4 \\ CRADD GENE: WHEN MEGALENCEPHALY MEETS LISSENCEPHALY}

ANDRE MIGUEL MIRANDA ${ }^{1}$, Joana Nunes ${ }^{1}$, Rita Quental ${ }^{2}$, Miguel Leao $^{2}$, Marta Vila Real ${ }^{3}$, Ana Filipa Geraldo ${ }^{1}$

${ }^{1}$ Diagnostic Neuroradiology Unit, Radiology Department, Centro Hospitalar Vila Nova de Gaia/Espinho, Vila Nova de Gaia, Portugal, ${ }^{2}$ Genetics Department, Centro Hospitalar e Universitario Sao Joao, Porto, Portugal, ${ }^{3}$ Neuropediatric Unit, Pediatric Department, Centro Hospitalar Vila Nova de Gaia/Espinho, Vila Nova de Gaia, Portugal

Keywords: lissencephaly, malformation, pachygyria

Introduction: Malformations of cortical development are a group of rare disorders commonly manifesting with developmental delay and seizures. Lissencephaly (LIS) spectrum disorders are associated with deficient neuronal migration and abnormal formation of cerebral convolutions, characterized by thickened cortex and gyral abnormality from agyria to oligogyria. So far, more than 20 lissencephaly-related genes have been discovered and affected patients nearly always present with normocephaly or microcephaly.

Results: A 4-year-old boy presented with mild developmental delay. Neurological examination revealed macrocephaly, divergent left strabism and delayed language development. Electroencephalogram detected subclinical paroxystic activity. Neuroimaging depicted megalencephaly and mild increased cortical thickening in the frontotemporal region bilaterally, associated with subtle reduction of sulcation/gyration pattern, in keeping with anterior-predominant "thin" lissencephaly. Coincidental findings included hypoplasia of the anterior commissure and mild callosal dysmorphism. Genetic analysis identified the variant c. $431 \mathrm{~A}>\mathrm{G}$ p.(Asp144Gly) in homozygosity in CRADD gene.
Discussion: CRADD gene encodes an adaptor protein implicated in caspase-2-mediated neuronal apoptosis during brain development. Pathogenic variants result in hallmark frontotemporal predominant pachygyria with mild cortical thickening. Severity of intellectual disability and EEG abnormalities may reflect variant pathogenicity and additional genetic or environmental factors. Notably, the unique association of macrocephaly/megalencephaly with LIS, not previously described in the remaining genes associated with LIS spectrum, configures a highly suggestive genotype-phenotype correlation that both radiologists and clinicians should promptly recognize.

4-P5

DIFFUSION-WEIGHTED MRI FINDINGS IN EARLY-ONSET VANISHING WHITE MATTER DISEASE: A CASE REPORT

JOAO ANIBAL SARAIVA ${ }^{1}$, Mariana Ribeiro Santos ${ }^{1}$, Sofia Queiros de Almeida Xavier $^{1}$, Joao Soares-Fernandes ${ }^{1}$

${ }^{1}$ Hospital de Braga, Neuroradiology Department, Braga, Portugal

Keywords: diffusion-weighted imaging, EIF2B5 gene, leukodystrophy, vanishing white matter disease

Vanishing white matter disease (VWMD) is one of the most common leukodystrophies, pathogenetically defined as an astrocytopathy caused by recessive mutations in any of the five genes encoding the subunits of eukaryotic translation initiation factor 2B (EIF2B1-5), more frequently missense variants in the EIF2B5 gene. Clinical course of VWMD is chronic-progressive with superimposed stress-provoked episodes of neurological deterioration. Prognosis is highly variable, reflecting a wellestablished genotype-phenotype correlation; most patients are compound heterozygous for 2 mutations, each one classified according to its severity and both contributing to overall phenotype. To the extent of our knowledge, MRI findings in VWMD have not been evaluated as potential surrogates of genotype severity.

A 2-year-old female, normocephalic child was admitted to inpatient care to further investigate worsening gait abnormality after a recent fall from standing height. Anamnesis included an upper respiratory tract infection (at 13 months) and delayed walking (18 months). As part of her workup, brain and lumbar MRI were performed, unveiling an extensive and symmetric cerebral hemispheric white matter involvement, partially sparring subcortical U-fibres, whose signal intensity resembled that of CSF on T1and T2-weighted images. On DWI, a clear-cut linear hyperintensity along the frontoparietal-temporal juxtacortical white matter was observed, consistent with active disease. Cystic transformation was noticeable in peritrigonal and frontal subcortical regions, and a "tigroid" pattern could be seen perpendicularly to ventricular walls, as well as two symmetrical punctiform high T2-weighted signal intensities in pontine tegmentum. Both optic nerves were mildly atrophic. Basal ganglia, thalami, and pyramidal tracts were spared and there was no contrast enhancement, neither in cranial nerves nor cauda equina. Spectra from centrum semiovale showed a prominent choline (Cho) peak with a smaller creatine peak (compatible with high cellular membrane turnover) and one peak around $3.80 \mathrm{ppm}$ possibly related to glucose (normally present in CSF). VHMD diagnosis was suggested and later confirmed by Sanger sequencing, denoting a mutated EIF2B5 gene, specifically p.Arg315His in the compound-heterozygous state; this mutation correlates with early onset and faster disease progression. By the age of 4 , patient was unable to walk, had a bilateral visual acuity of 1/10 (decimal scale) and dysphagia for liquids.

Currently, no effective treatment is available for VWMD. MRI findings may be useful not just to get a presumptive VWMD diagnosis and promptly prevent exposure to stress triggers, but also to detect signs of active demyelination, most notably on DWI, in an attempt to predict rapidly progressive disease. 


\section{4-P6}

NEUROIMAGING IN AICARDI-GOUTIERES SYNDROME TYPE 5 - CASE REPORT

\section{$\underline{\text { IVANA MARKOVIC }}^{1}$, Bosanka Jocic Jakubi ${ }^{2}$, Almundher Abdullah Ali Al-Maawali}

${ }^{1}$ Department of Radiology and Molecular Imaging, Sultan Qaboos University Hospital, Muscat, Oman, ${ }^{2}$ CHD Neurology, Sultan Qaboos University Hospital, Muscat, Oman, ${ }^{3}$ Clinical Genetics, Sultan Qaboos University Hospital, Muscat, Oman

Keywords: rare, leukodystrophy, inherited

Introduction: Aicardi-Goutieres syndrome is rare neurodegenerative hereditary disease with systemic and central nervous system inflammatory syndrome characterized with encephalopathy, vasculopathy and hepatosplenomegaly. All published studies have shown unknown or a prevalence of 1-5 per 10,000 live births with no gender predilection.

Methods \& Results: We present the case of two years old male infant admitted to our hospital at the first month of life for further diagnostic evaluation and therapy due to failure to thrive, global developmental delay, microcephaly, poor vision, upper and lower limbs spasticity along persisting associated abnormal body movements, left ventricular hypertrophy and chronic constipation with vomiting (severe GERD) on medications. Family history was positive regarding to consanguineous parents and cerebral palsy diagnosed in older sister. Child was underwent to US, CT and MRI examination apart from all other coordinated planned diagnostic procedures and clinical examinations including genetic testing.

The initial (performed at the age of one month) and follow up MRI examination (performed at the age of 11 months) were reported with following findings: diffuse signal abnormalities of supratentorial white matter in the way of leukodystrophia developing later as leukomalacia with cystic areas in occipital and temporal lobes following by certain but atrophy changes; intracerebral vasculopathy presented with bilateral multisegmental intracranial arterial narrowing stenosis; presence of punctuate and confluent bilateral extensive calcifications within periventricular and subcortical white matter including basal ganglia. Finding of dense and rock-like calcifications was confirmed by CT.

Performed genetic testing confirmed homozygous gene mutation SAMHD1 associated with AGS type 5.

Discussion \& Conclusion: This multisystemic disorder or syndrome named after French pediatric neurologists Jean Aicardi and Francoise Goutieres described the first case in 1984.

During the time, disease evolution is characterized usually with brain atrophy, in some cases with associated cerebellar and brain stem atrophy. Arteriopathy is prominent feature of SAMHD1 mutations with possible aneurysms, stenoses and like moya-moya pattern of vessels.

AGS is an inherited leukodystrophy with calcifying microangiopathy and abnormal myelination. There is certain possible risk to miss diagnosis of this entity due to extensive brain calcifications.

The main differential diagnosis include MICS also known as "pseudoTORCH" syndrome, TORCH congenital infections, Band-like calcification PMG and Cockayine syndrome.

Final diagnosis has to be confirmed by genetic testing due to mutations of seven different genes associated with different types of AGS.

\section{4-P7}

STURGE WEBER TYPE 3 - A NEUROCUTANEOUS SYNDROME WITHOUT SKIN MANIFESTATIONS
ANDRE ARAUJO $^{1}$, Andre Miguel Miranda ${ }^{1}$, Luis Cardoso ${ }^{1}$, Fatima Santos $^{2}$, Joana Nunes ${ }^{1}$

${ }^{1}$ Imagiology Department, Neuroradiology Unit, Centro Hospitalar de Vila Nova de Gaia/Espinho, Portugal, ${ }^{2}$ Pediatrics Department, Centro Hospitalar de Vila Nova de Gaia/Espinho, Portugal

Keywords: phakomatosis, Sturge Weber, angiomatosis

Introduction: Sturge-Weber syndrome is a relatively rare phakomatosis characterized by an encephalotrigeminal angiomatosis with a persistent fetal vasculature and facial and cerebral venous stasis. A somatic mutation in GNAQ has been described as a potential cause.

Patients may present with a facial nevus flammeus (port-wine stain), seizures, developmental delay, or stroke-like episodes. A unilateral port-wine stain in the trigeminal nerve territory is characteristic, can be an isolated finding and it's absence does not exclude Sturge-Weber syndrome.

On imaging, venous stasis and flow redirection to the deep vessels of the brain can be found. Accelerated myelination and increased regional perfusion are typical findings in neonates. Pial angiomatosis is usually seen as a serpentine contrast enhancement following the cortical gyri. With progression, there is ensuing focal atrophy of the affected areas.

Methods: A clinical case of an infant presenting with focal seizures.

Results: A 2 months-old female without perinatal relevant history is brought to the emergency department due to 4 episodes of focal crises in the last 12 hours, with clonic movements in the left inferior limb (one with extension to the ipsilateral superior limb), with preserved conscious state and complete recovery. No fever or other accompanying symptoms. Neurological and physical examination were unremarkable. Transfontanellar ultrasound and EEG were normal. Brain MRI revealed a signal alteration in the pre-central gyrus, with discrete spontaneous T1 hypersignal, marked T2 hyposignal, hyposignal in the ADC map (without evident hypersignal in DWI-B1000), and volume loss. There were no parenchymal or extra-axial calcifications. After gadolinium administration there was marked leptomeningeal enhancement and diffuse leptomeningeal hypertrophy on both cerebral hemispheres, with particular prominence in the medial parietal sulci of the right convexity.

Discussion: vThe clinical presentation and MRI findings reflect leptomeningeal/pial angiomatosis that could be framed in the context of encephalotrigeminal angiomatosis/Sturge-Weber Syndrome. In the absence of cutaneous stigmata, one should consider Sturge Weber Syndrome type III - isolated leptomeningeal angioma. In this context, the signal abnormalities probably reflect accelerated myelination. On the differential diagnosis one should consider an infectious etiology, inherited metabolism disorders, cortical developmental malformations, other phakomatosis such as Tuberous Sclerosis or PHACE-S and epileptic syndromes, all unlikely regarding the clinical and imaging presentation. The child evolved favorably and was seizure-free under anticonvulsants at follow-up.

Conclusion: One should be aware of Sturge Weber Syndrome type 3 in which the imaging findings play a major role in the absence of other clinical, laboratory and imaging findings.

\section{SPINE}

\section{5-P1}

MAGNETIC RESONANCE IMAGING EVALUATION OF REFRACTORY CHRONIC INFLAMMATORY DEMYELINATING POLYNEUROPATHY (CIDP): A CASE REPORT 
$\underline{\text { AFIF OMAR }}^{1}$, N.H. Rahim ${ }^{1}$, S.C. Muniandy ${ }^{1}$, N.R. Mohd Zain ${ }^{1}$

${ }^{1}$ Radiology Department, Kuala Lumpur Hospital, Kuala Lumpur, Malaysia

Keywords: Magnetic Resonance Imaging, Refractory Chronic Inflammatory Demyelinating Polyneuropathy (Cidp), Peripheral Nerve Demyelinating Disease

Chronic inflammatory demyelinating polyneuropathy (CIDP) is an acquired peripheral nerve demyelinating disease of presumed autoimmune aetiology, commonly characterized clinically by a progressive or relapsing course of many months to years of symptoms similar to compressive myelopathy. The diagnostic criteria for CIDP are usually based on clinical presentation, electrophysiological findings (nerve conduction study) and nerve biopsy. MRI features for patients with refractory chronic inflammatory demyelinating polyneuropathy are seldom reported. Here, we report a case of 27 years old gentleman with refractory chronic inflammatory demyelinating polyneuropathy presented with history of worsening upper and lower limb numbness, gradual weakness in almost 3 years of both proximal and distal musculature associated with areflexia and hypoesthesia. Patient was initially subjected to IV Rituximab, followed by IV immunoglobulin and plasma exchange. MRI demonstrates massive thickening with T2/STIR hyperintense signal as well as mild enhancement of cervical nerve roots with bilateral brachial plexus, dorsal and intercostal nerve as well as lumbar plexus involvement. These MRI features are characteristic and favoured a diagnosis of hypertrophic polyneuropathy like chronic inflammatory demyelinating polyneuropathy (CIDP) with a prolonged clinical course. Diagnosing CIDP enabled the commencement of definitive treatment which led to a good recovery. Nerve conduction studies are considered essential for a definite diagnosis, but poor performance and misinterpretation of the results may lead to misdiagnosis. MRI could be a very helpful tool in diagnosis of CIDP.

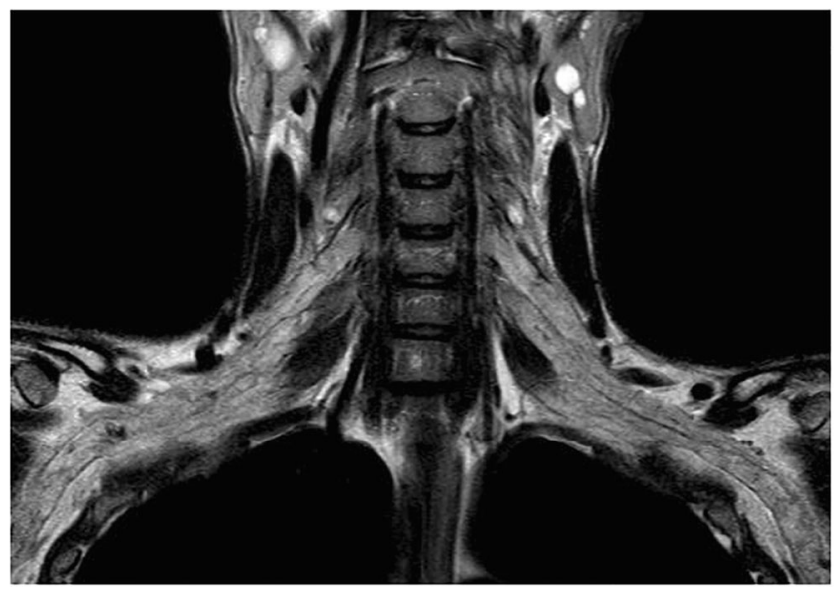

5-P2

"WHAT'S WRONG WITH MY HAND?". HIRAYAMA DISEASE: A CASE REPORT

\section{$\underline{\text { AFIF OMAR }}^{1}$, Khairulanuar Saad @ Abdul Halim $^{1}$}

${ }^{1}$ Radiology Department, Kuala Lumpur Hospital, Kuala Lumpur, Malaysia

Keywords: Hirayama Disease, Cervical, Juvenile Muscular Atrophy

Hirayama's disease (HD) is a rare condition characterized by asymmetric muscle weakness and atrophy with propensity to involvement of lower cervical region. The disease is also known as "juvenile muscular atrophy" of distal upper limb extremity. A 20-year-old male who presented with a history of insidious onset of weakness and muscle wasting in both upper extremities through 5 years duration. Apart from that, he also experienced numbness and hypoesthesia. There was no preceding trauma, febrile illness, poliomyelitis or exposure to toxins or heavy metals in the past. Neurological examination revealed a mixed pattern of upper and lower motor neuron deficit affecting both upper extremities with marked weakness and muscle wasting, sparing the brachioradialis muscles. Power of the wrists and hands of both upper limbs were reduced. Nerve conduction study was inconclusive. Further ultrasound study was unremarkable. Dynamic MRI of cervical spine T2W MR cervical in normal position demonstrates $\mathrm{C} 5$ to $\mathrm{T} 1$ level spinal cord atrophy. The posterior dural sac is located close to the posterior wall of spinal canal. T2W MR cervical in flexion position demonstrates forward shifting of posterior dural sac from $\mathrm{C} 4$ to $\mathrm{T} 1$ level, resulting in widened laminodural space. In conclusion, high clinical suspicion of HD is necessary in young patients with insidious onset of weakness of the hand and forearm muscles. Flexion MR imaging sequences is important to establish the diagnosis as it typically illustrates forward shifting of posterior dural sac or widening of laminodural space at lower cervical region.

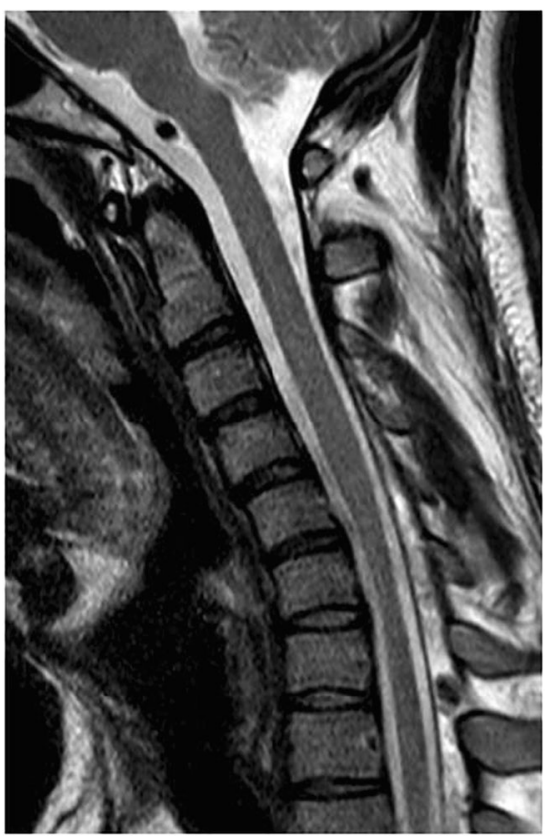

\section{5-P3}

WHAT YOU REALLY NEED TO KNOW ABOUT INFECTIOUS SPONDYLODISCITIS: A PICTORIAL REVIEW OF THE IMAGING FINDINGS

\section{$\underline{\text { ONUR SIMSEK }}^{1}$, Burcu Savran $^{1}$, Nazan Ciledag $^{1}$, Leyla Ince $^{1}$}

${ }^{1}$ Dr. Abdurrahman Yurtaslan Ankara Oncology Training and Research Hospital, University of Health Sciences, Ankara, Turkey

Keywords: discitis, osteomyelitis, epidural abscess, spine, tuberculosis

\section{Purpose \& Learning Objectives:}

- To provide a pictorial review of infectious spondylodiscitis in the light of current literature

- To provide examples of typical imaging features of different organisms 
- To review common mimics and clues for the differential diagnosis of infectious spondylodiscitis

- To discuss main complications

\section{Approach:}

- Infectious spondylodiscitis cases from our centre were presented to demonstrate different infiltration characters of various organisms.

- Additional handcrafted illustrations and three dimensional surface rendering images were added to provide a better learning experience.

- For each case, clinical information and imaging features were used to establish diagnosis.

Background: Infectious spondylitis is regarded as a serious and morbid disease. The disease may occur with hematogenous spread, or it may develop due to prior surgery or surrounding soft tissue infections. Early diagnosis is important since it might cause complications such as epidural abscess. Clinical symptoms of infectious spondylodiscitis are mostly nonspesific such as fever and pain, thus imaging has a very important role to suspect the disease. In most cases diagnosis of infectious spondylodiscitis is suspected after MRI of the spine. Blood culture and CT-guided biopsy are used for further evaluations.

The most common causative organism of infectious spondylodiscitis is Staphylococcus spp. The other important organisms may be counted as brucellosis, tuberculosis, fungi and pseudomonas. Although these spondylodiscitis have similar imaging features, some clues may guide us in the differential diagnosis. Our review focuses on imaging clues that point to different causative organisms.

Cases:

- Pyogenic spondylodiscitis

- Brucellar spondylodiscitis

- Tuberculous spondylodiscitis

- Fungal spondylodiscitis

- Complications: Epidural abscess

- Complications: Paraspinal abscess

- Mimics of infectious spondylodiscitis: Schmorl node

- Mimics of infectious spondylodiscitis: Modick type 1 degenerative changes

- Mimics of infectious spondylodiscitis: Langerhans Cell Histiocytosis

\section{Conclusion:}

- Infectious spondylodiscitis is associated with morbidity and early diagnosis might prevent complications

- MRI of the spine is a helpful tool in diagnosis of infectious spondylodiscitis and differentiating it from other likely diagnosis.

- Some pathogens present specific imaging findings which can be useful to guide empirical treatment.
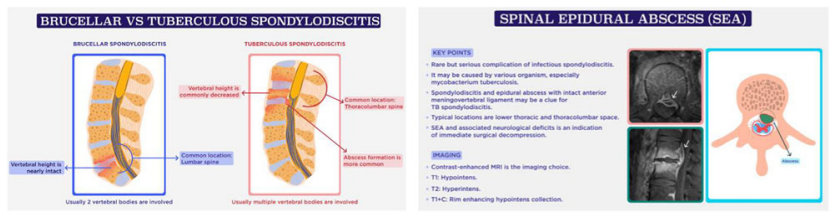

5-P4

CSF, YOU SHOULD NOT BE HERE! HOW TO RECOGNIZE CSF LEAK IN CT-CISTERNOGRAPHY AND CT-MYELOGRAPHY IMAGES

MIKA SHIBUYA SETUGUTI ${ }^{1}$, Daniel Takeshi Setuguti ${ }^{1}$, Marcio Nattan Portes Souza $^{1}$, Marcelo Calderaro ${ }^{1}$, Rita Cassia Maciel Pincerato ${ }^{1}$

${ }^{1}$ Samaritano Hospital - UHG Americas, Sao Paulo, SP, Brazil

Keywords: CT myelography, CSF leak, CSF venous fistula

Introduction: CSF leak results from direct communication of the CSF to extra-cranial / extra-spinal space, and may have different etiologies, clinical manifestation and image presentation depending on their location. This pictorial essay aims to demonstrate the main locations and imaging findings of CSF leaks in CT-cisternography and CT-myelography.

Methods: We illustrate in a pictorial essay the main imaging findings of the cranial and spinal cerebrospinal fluid leakage in myelotomography and cisternography exams.

Results: Most frequently, CSF leaks at skull base are located in the anterior cranial fossa, and it is necessary to look for extravasation of iodinated contrast especially in the lateral lamella of the cribriform plate. Other sites of bone fragility include the frontal sinus walls, sella turcica and sphenoid sinuses. In spinal CSF leaks, most of them are located in the thoracic spine and thoracolumbar transition, and their appearance varies from patient to patient and depends on the cause of the leak. The extravasation of iodinated contrast may have a broad base along the thecal sac or along the axilla of the nerve root (when related to meningeal diverticula), a extensive epidural CSF collection (associated a ventral dural tear), and, sometimes, associated a contrast medium of a paraspinal vascular structure along the lateral aspect of the vertebral body and early renal contrast on CT-myelography such as in CSF venous fistulas.

Discussion \& Conclusion: CSF leaks in the intracranial compartment occur through a bone defect at the skull base and are usually related to symptoms such as rhinorrhea/otorrhea. In turn, CSF leaks in the spinal compartment result from a dural defect by degenerative disc-vertebral changes and meningeal diverticula, or by spontaneous drainage into the venous compartment (CSF venous fistulas), and are commonly associated with orthostatic headache. Conventional non-invasive imaging methods, such as skull base CT and brain MRI, only show indirect findings of these types of leaks, often requiring the exact location of the dural defect for therapeutic success. Therefore, it's extremely important for radiologists to recognize the main locations and imaging findings of CSF leaks, which are often subtle.
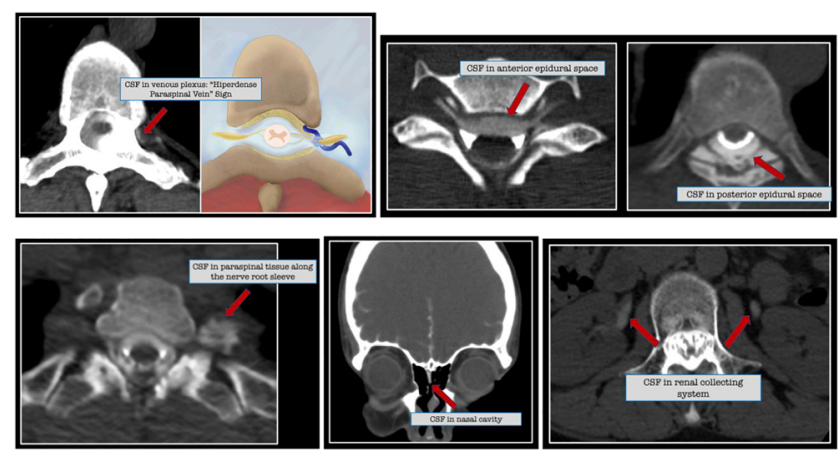


\section{5-P5}

BROWN TUMOR: A RARE CAUSE OF SPINAL CORD COMPRESSION

\section{MARIANA RIBEIRO SANTOS ${ }^{1}$, Joao Anibal Saraiva ${ }^{1}$, Miguel Quintas-Neves ${ }^{1}$, Angelo Carneiro ${ }^{1}$}

${ }^{1}$ Neuroradiology Department, Hospital de Braga, Braga, Portugal

Keywords: Brown tumor, Hyperparathyroidism, spinal cord compression

Introduction: Currently, the incidence of secondary hyperparathyroidism is increasing, probably due to the more effective treatment of patients with final stage chronic kidney disease (CKD). The brown tumor or osteitis fibrosa cystica is a benign lytic bone tumor related to hyperparathyroidism, that may rarely appear in the vertebrae and cause spinal cord compression.

Case report: A 41-year-old woman receiving long-term hemodialysis (6 years) due to hypertensive nephrosclerosis came to our hospital emergency department due to a 5-month clinical history of progressive weakness of the lower extremities, dorso-lumbar pain and sphincter incontinence. Neurological examination revealed spastic paraplegia, anesthesia and hyperreflexia of the lower extremities. Spine CT showed diffuse skeletal involvement by multiple mixed lytic/sclerotic lesions, mostly at the vertebral bodies; there was an expansible lesion with soft tissue density compressing the spinal cord at the level of T11, inducing a pathologic fracture of this vertebra. Subsequent MRI confirmed the spinal cord compression and consequent edema; the lesion was hypointense on all sequences.

Laboratory work-up revealed increased parathormone (PTH) serum levels (1310.26 pg/mL; normal: 18.5 - $88 \mathrm{pg} / \mathrm{mL}$ ), with unremarkable calcium and phosphorus. Dedicated ultrasound examination showed enlargement of the left parathyroid gland.

Pathological analysis of a biopsy of a first costal arch lytic lesion was compatible with a giant cell tumor. Therefore, final diagnosis of brown tumors in the setting of secondary hyperparathyroidism was made.

Subtotal parathyroidectomy was performed, with pathological analysis confirming gland hyperplasia. Spinal cord decompression was not performed due to the chronicity of symptoms.

After surgery, there was normal PTH serum levels.

Patient reported improvement from dorso-lumbar pain, but equal motor deficit.

Conclusion: This case depicts a rare entity that should be considered in the differential of spinal cord compression by expansile lesions, especially in female patients with final stage CKD. This unusual clinical presentation highlights the importance of performing careful monitorization of these patients, given that hyperparathyroidism is a treatable condition. Parathyroidectomy and medical treatment may be used together. Decompression surgery should be performed whenever neurologic deficits are still potentially reversible.

\section{5-P6}

TRAUMATIC VERTEBRAL FRACTURES: SAME MECHANISM OF INJURY, DIFFERENT FEATURES BY AGE GROUP - A CASE REPORT

\section{PEDRO S. BRANDAO ${ }^{1}$, Sofia Bettencourt ${ }^{1}$, Carla Conceicao $^{1}$}

${ }^{1}$ Department of Neuroradiology, Centro Hospitalar Universitario de Lisboa Central, Lisbon, Portugal
Keywords: spine, trauma, vertebral fractures, pediatric

Introduction: Although frequently related to osteoporosis, other pathological conditions and high-energy trauma can also lead to vertebral compression fractures (VCF). Traumatic VCF usually result from axial loading mechanisms, with traffic-related incidents and falls being the most common causes. In the pediatric population, fractures of ossified vertebral structures are rare under 8 years of age, and its anatomical distribution is known to differ from adults, due to skeletal development.

We describe an illustrating case of a child and an adult exposed to the same traumatic spinal axial load yet showing different patterns of injuries.

Case Report: A 7-year-old male and his father were admitted to the emergency department after falling from a height of 6 meters inside their vehicle in a car elevator.

The child presented with cervicothoracic spinal tenderness, and his neurological assessment was normal. Initial CT and additional MRI scans demonstrated VCF affecting T3-T11 and L3-L4 (anterior vertebral body wedging of T4-T9, particularly at T5 and T6), without the involvement of the posterior vertebral body wall. An accompanying non-compressive posterior acute epidural hematoma from T1-T9 was completely reabsorbed on follow-up MRI scan, performed eleven days later. Since there were no instability or neurological deficits, a thoracolumbar orthosis was recommended for 6 weeks in a conservative approach.

The 56-year-old male experienced pain referred to the thoracic and abdominal regions, without neurovascular compromise on physical examination. Whole spine CT scan showed burst fractures of L2 and L5 with retropulsed fragments causing spinal canal stenosis. He was subsequently submitted to surgical L1-S1 arthrodesis and bilateral decompressive laminectomy of L2, with clinical improvement. Initial CT also found simple VCF of T11, T12, L3, and L4, and fractures of the neural arch of L2, which were managed conservatively.

Discussion \& Conclusion: Anatomy and biomechanics of the spine change as it matures, which determines particular age-dependent features of vertebral fractures.

The unique circumstances of this case highlight that the same mechanism of injury acting simultaneously on two individuals of different age groups produces specific patterns of spinal involvement. In children, thoracolumbar traumatic fractures tend to affect more vertebrae and occur in higher thoracic levels, but the somatic deformity is usually less prominent than in adults, as shown.

5-P7

COVID-19-ASSOCIATED LONGITUDINAL EXTENSIVE TRANSVERSE MYELITIS

ANA CAROLINA BRAGA CHAVES ${ }^{1}$, Pedro Barradas ${ }^{1}$, Fabio Gomes $^{2}$, Pedro Melo Freitas ${ }^{1}$, Silvia Carvalho ${ }^{1}$, Ines Correia ${ }^{2}$, Cesar Nunes $^{1}$

${ }^{1}$ Medical Imaging Department - Neuroradiology, Coimbra University Hospital Centre, Coimbra, Portugal, ${ }^{2}$ Neurology Department Coimbra University Hospital Centre, Coimbra, Portugal

Keywords: LETM, SARS-CoV-2, COVID-19, MRI, paraparesis

Introduction: Despite mainly showing respiratory manifestations, severe acute respiratory syndrome coronavirus 2 (SARS-CoV-2) has been shown capable of affecting the nervous system, either directly through specific receptors in neuronal and glial cells, or through indirect processes such as inducing severe immune responses, causing encephalitis, or affecting the vascular system, leading to acute ischemic stroke. One rarely described consequence of Coronavirus Disease 19 (COVID-19) is acute 
transverse myelitis which, when present, usually seems to manifest days to weeks after infection, with a latency period suggestive of postinfectious origin. Patients may present with bilateral dysfunction of motor, sensory or autonomic functions, with magnetic resonance image (MRI) helping to confirm the diagnosis by showing signs of spinal cord inflammation.

Methods: We report a case of longitudinal extensive transverse myelitis (LETM) post COVID-19 and perform an analysis of the literature.

Results: An 84-year-old man who presented to the emergency department with acute urinary retention, paraparesis and decrease in sensation bellow the T5 level. Two weeks before, the patient had fever and myalgia and was diagnosed with COVID-19. Lymphopenia and high c-reactive protein plasma levels were noted. Cerebrospinal fluid (CSF) analysis revealed WBC $27 / \mu \mathrm{L}$, protein $90(15-40 \mathrm{mg} / \mathrm{dL})$ and was positive for SARS-CoV-2 on reverse transcription polymerase chain reaction. MRI of the cervical and thoracic spine showed an extensive T2 hyperintense lesion of the spinal cord spanning from $\mathrm{C} 1$ to $\mathrm{T} 10$, with moderately diffuse swelling of the involved segment. The patient was started on pulse methylprednisolone and plasma exchange and showed mild improvement of paraparesis. A follow-up MRI at 2-weeks demonstrated diminished signal span and T2 intensity, with decreased spinal cord edema.

Conclusion: This case describes an uncommon neurological manifestation of COVID-19. To our knowledge this is one of the most extensive cases of COVID-19-associated myelitis described. Neurologic symptoms should be promptly recognized among COVID-19 patients and should alert the clinician to potential complications. Early diagnosis of myelitis is imperative and spinal cord MRI findings are key to establish the diagnosis. Our case highlights the importance of neuroimage in the study of these patients, when the suspicion of neurologic complications arises.

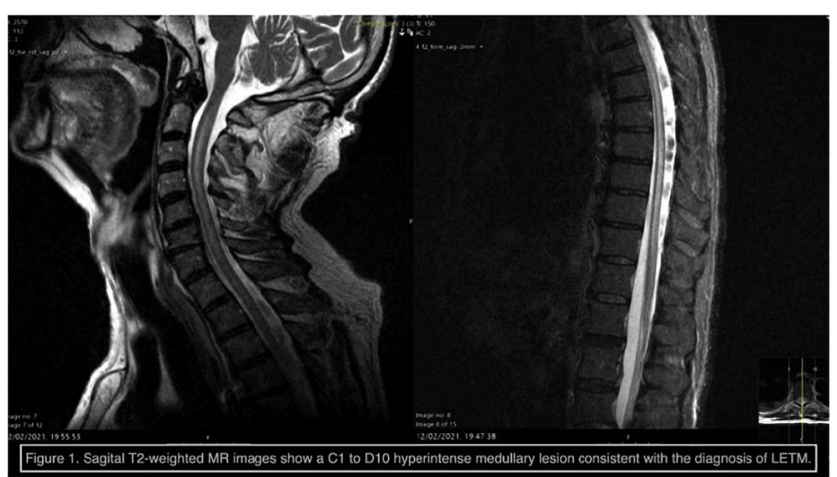

5-P8

SPINAL INTRADURAL EXTRAMEDULLARY CYSTIC LESIONS

$\underline{\text { BRUNO CUNHA }}^{1}$, Carla Conceicao $^{1}$

${ }^{1}$ Neuroradiology Department, Centro Hospitalar Universitario de Lisboa Central; NOVA Medical School, Universidade Nova de Lisboa - Lisboa, Portugal

Keywords: Spine, Intradural extramedullary lesions, Spinal tumors, MR imaging, CT myelography, Intradural arachnoid cyst, Dermoid cyst, Epidermoid cyst, Schwannoma, Lipoma, Dorsal thoracic arachnoid web, Spinal cord herniation, Subdural hematoma, Subdural abscess

Introduction: A wide variety of conditions may present as intraspinal cystic lesions. Clinical presentation is usually non-specific and imaging studies are frequently key in the diagnosis and treatment planning of these lesions. As with other intraspinal lesions, localization of intraspinal cystic lesions as extradural, intradural extramedullary and intramedullary is an important first step in the evaluation of these lesions, helping to narrow a differential diagnosis. This pictorial review focuses on intradural extramedullary cystic lesions.

Methods: We provide a pictorial review of spinal intradural extramedullary cystic lesions and depict the key and occasionally distinctive imaging findings that can guide the neuroradiologist towards an accurate diagnosis.

Results: Intradural extramedullary cystic lesions include congenital/ developmental disorders, such as intradural arachnoid cyst, dermoid and epidermoid cyst and neuroenteric cyst, post-traumatic/post-surgical collections, such as subdural hematoma or abscess, infectious diseases, such as neurocysticercosis, spinal tumors such as cystic schwannoma, and other miscellaneous lesions, such as subdural collections in CSF hypotension. Lipoma and teratoma may mimic cystic lesions on T2weighted images. While imaging features may be overlapping, clinical background, concurring abnormalities and MR signal characteristics provide important clues for diagnosis. T1 hyperintensity occurs in the presence of fatty tissue in lipoma and teratoma and occasionally in dermoid cyst, as well as in subacute hemorrhage. Diffusion restriction on DWI is more likely in epidermoid and is also present in subdural abscess. Contrast-enhancement of the cystic walls raises suspicion for infectious or tumoral lesions. Additionally, the widened CSF space and focal spinal cord indentation present on dorsal thoracic arachnoid webs and spinal cord herniation (scalpel sign) may be difficult to distinguish from CSFisointense space-occupying lesions. Cord tissue protrusion through a dural defect may be appreciated at high-resolution thin-section MR imaging. Unimpeded CSF pulsation artifacts within a widened CSF space helps to exclude a space-occupying lesion, while a filling defect at CT myelography can help support a space-occupying lesion.

Discussion \& Conclusion: Several conditions present as spinal intradural extramedullary cystic lesions, frequently with overlapping imaging characteristics. A confident imaging diagnosis is often challenging. Nonetheless, knowledge of the most common causes and awareness for the key and distinctive imaging features can guide the neuroradiologist in the differential diagnosis. MR imaging is the technique of choice in presurgical evaluation, providing an accurate location and lesion characterization, as well as in post-surgery follow-up. High-resolution thin-section MR imaging and CT myelography may also provide important distinctive information in specific cases.

\section{AUTHORS INDEX}

\begin{tabular}{lll}
\hline $\begin{array}{l}\text { Lastname } \\
\text { Abdullah Ali } \\
\text { Al-Maawali }\end{array}$ & Firstname & Abstract Number \\
Acu & Almundher & 4-P6 \\
Akansel & Leyla & $1-\mathrm{O} 14,1-\mathrm{P} 59$ \\
Al Koteesh & Gur & 1-P9 \\
Al Zahmi & Jamal & 1-P11, 1-P12, \\
& & $1-\mathrm{P} 13,1-\mathrm{P} 15$ \\
Aldhebaib & Fatmah & $1-\mathrm{P} 11,1-\mathrm{P} 12$, \\
Alexoudi & & $1-\mathrm{P} 13,1-\mathrm{P} 15$ \\
Alhasan & Ali & $1-\mathrm{P} 8$ \\
Almansoori & Athanasia & $1-\mathrm{P} 57$ \\
& Hamza & $1-\mathrm{O} 16$ \\
& Taleb M. & $1-\mathrm{P} 11,1-\mathrm{P} 12$, \\
& & $1-\mathrm{P} 13,1-\mathrm{P} 15$
\end{tabular}




\begin{tabular}{|c|c|c|c|c|c|}
\hline Almeida & Francisco & $1-\mathrm{O} 6$ & Bink & Andrea & $1-\mathrm{P} 42$ \\
\hline Almeida & Goncalo & 1-P18 & Binti Mat Nor & Khariah & 1-P16 \\
\hline Almeida & Joao & 3-P8 & Binti Rosani & Emy Saera & 1-P16 \\
\hline Alotaibi & Abdulmajeed & 1-P5, 1P8 & Boado & Victoria & $1-\mathrm{O} 15$ \\
\hline Altoe & Ananda & 1-P36 & Boeckenhoff & Annette & 1-P7 \\
\hline Altokhis & Amjad & 1-P5, 1-P8 & Bondarenko & Vladsilav & $1-\mathrm{P} 21$ \\
\hline Altundag & Aytug & $3-\mathrm{P} 1$ & Boskos & Christos & 1-P56 \\
\hline Alves Alves & Viriato & 1-P51 & Botelho & Luis & 2-P4 \\
\hline Alves Fonseca & Ana Paula & 1-P52, 1-P54, 1-P61 & Boto & Jose & 1-O1, 1-P66 \\
\hline Ambegaonkar & Gautam & 4-P3 & Boxberg & Frederik & 2-P9 \\
\hline Amer & Ahmad & $1-\mathrm{O} 16$ & Bozzao & Alessandro & 1-P10, 1-P14 \\
\hline Antunes Naves & Erica & 1-P52, 1-P54, 1-P61 & Braga Chaves & Ana Carolina & $5-\mathrm{P} 7$ \\
\hline Aparecida Felipe & Marcela & $1-\mathrm{P} 38$ & Branco & Gabriel & $1-\mathrm{P} 23$ \\
\hline Aparici Robles & Fernando & 1-P55 & Brandao & Pedro S. & 5-O1, 5-P6 \\
\hline Arango-Lasprilla & Juan Carlos & $1-\mathrm{O} 15$ & Brassel & Friedhelm & 2-P9 \\
\hline Araujo & Andre & 4-P7 & Braz & Ana & 1-P62 \\
\hline Araujo & Luziany & 1-P47 & Brennan & Paul & 2-P8 \\
\hline Ardellier & Francois-Daniel & 1-P7 & Brescia Morra & Vincenzo & 1-O9, 1-P40, \\
\hline Arnold & Marcel & $1-\mathrm{O} 12$ & & & 1-P41, 1-P43 \\
\hline Arzanauskaite & Monica & 1-P30 & Brina & Olivier & $1-\mathrm{P} 20$ \\
\hline Asadi & Hamed & 2-P1 & Brinjikji & Waleed & $3-\mathrm{O} 1$ \\
\hline Asavoaie & Carmen & 4-P1 & Brotman & Ryan & $1-\mathrm{P} 65$ \\
\hline Ashok & Abhishekh & 4-P3 & Brunetti & Arturo & 1-O9, 1-P40, \\
\hline Auger & Cristina & 1-O8, 1-P33 & & & 1-P-41,1P-43 \\
\hline Avsenik & Jernej & 1-P26 & Bruno & Federico & 1-O11, 5-O3, 2-P6 \\
\hline Aygun & Berna & 1-P48 & Byun & Jun Soo & $1-\mathrm{O} 4$ \\
\hline Azevedo & Paula & 3-P3 & Cabrera-Zubizarreta & Alberto & $1-\mathrm{O} 15$ \\
\hline Baar & Ingrid & $1-\mathrm{P} 28$ & Calderaro & Marcelo & $5-\mathrm{P} 4$ \\
\hline Baid & Ujjwal & $1-\mathrm{O} 10$ & Camara & Estela & 1-P35 \\
\hline Bakas & Spyridon & $1-\mathrm{O} 10$ & Camins-Simon & Angels & 1-P35 \\
\hline Balci & Sibel & 1-P9 & Campeau & Norbert G. & $3-\mathrm{O} 1$ \\
\hline Baptista & Mariana & $5-\mathrm{O} 1$ & Canhao & Patricia & 1-P29 \\
\hline Baptista & Miguel & $1-\mathrm{P} 24$ & Cappelle & Sarah & $1-\mathrm{P} 25$ \\
\hline Baptista De & Aline Almeida & 1-P52, 1-P54, 1-P61 & Cardoso & Luis & 4-P7 \\
\hline Oliveira Salgado & & & Cardoso Diogo & Mariana & $3-\mathrm{P9}$ \\
\hline Barata Tavares & Joana & $1-\mathrm{P} 23$ & Carmo & Isabel & $1-\mathrm{P} 23$ \\
\hline Barkhof & Frederik & $1-\mathrm{O} 2$ & Carneiro & Angelo & 5-P5 \\
\hline Barras & Christen D. & 2-P1 & Carreres-Polo & Joan & 1-P55 \\
\hline Barsi & Peter & 1-P50 & Carvalho & Joao & 1-P37 \\
\hline Batista & Francisca Sena & 2-P7, 3-P9 & Carvalho & Rui & 1-P18 \\
\hline Beall & Doug & $5-\mathrm{O} 4$ & Carvalho & Silvia & $5-\mathrm{P} 7$ \\
\hline Belghali & Maroua & 1-P11, 1-P12, & Caschera & Luca & $1-\mathrm{P} 45$ \\
\hline & & 1-P13, 1-P15 & Casimiro & Carlos & 1-P22, 1-P23 \\
\hline Bem Gomes & Pedro & 2-O2, 1-P24, 2-P5 & Cassia Maciel & Rita & 1-P52, 1-P54, \\
\hline Benito-Sanchez & Itziar & $1-\mathrm{O} 15$ & Pincerato & & 1-P61, 5-P4 \\
\hline Benson & John & $3-\mathrm{O} 1$ & Castaldi & Antonio & $1-\mathrm{P} 49$ \\
\hline Berdalin & Alexander & 1-O5, 1-P21 & Castanedo Vazquez & David & 1-P63 \\
\hline Berges & Olivier & 3-O2, 3-P5 & Casteras & Ana & $1-\mathrm{O} 8$ \\
\hline Bernava & Gianmarco & 2-O1, 2-O3, 2-P3 & Castorani & Giulia & $1-\mathrm{P} 45$ \\
\hline Bettencourt & Sofia & 5-O1, 5-P6 & Catalucci & Alessia & 2-P6 \\
\hline Biagetti & Betina & $1-\mathrm{O} 8$ & Charbonneau & Frederique & 1-O7, 3-O3, 3-P5 \\
\hline Bilello & Michel & $1-\mathrm{O} 10$ & Charykova & Inna & $\begin{array}{l}\text { 1-P11, 1-P12, } \\
\text { 1-P13, 1-P15 }\end{array}$ \\
\hline
\end{tabular}




\begin{tabular}{|c|c|c|c|c|c|}
\hline Chen & Andrew & $1-\mathrm{P} 4$ & Do Amaral & Lazaro Luis Faria & 1-P19, 1-P34 \\
\hline Chiu & Lucinda & $1-\mathrm{P} 4$ & Dorigatti Soldatelli & Matheus & 1-P19, 1-P34, 1-P38 \\
\hline Choi & David & $1-\mathrm{P} 4$ & Drake-Perez & Marta & 1-P63 \\
\hline Chong & Winston & 2-P1 & Duenas Cabrera & Ferdinand & 1-P36, 1-P39 \\
\hline Christodoulou & Evangelia & $1-\mathrm{P} 27$ & Filho & & \\
\hline $\mathrm{Chu}$ & Yin Lun Edward & 2-P2 & Dumitriu La Grange & Daniela & 2-O3, 1-P66 \\
\hline Chung & Mi Sun & $1-\mathrm{O} 4$ & Duron & Loic & $3-\mathrm{O} 2,3-\mathrm{O} 3$ \\
\hline Ciledag & Nazan & $5-\mathrm{P} 3$ & Dy Choa-Go & Joanna Marie & 1-P30 \\
\hline Clavel & Gaelle & $1-07$ & Eckel & Laurence J. & $3-\mathrm{O} 1$ \\
\hline Cobo Ruiz & Teresa & 1-P63 & Elefante & Andrea & $1-\mathrm{O} 9$ \\
\hline Cocozza & Sirio & 1-O9, 1-P40, & $\begin{array}{c}\text { Emelijanovich } \\
\text { Olyushin }\end{array}$ & Victor & 1-P1, 1-P2 \\
\hline & & 1-P-41, 1-P43 & Endrikat & Jan & 1-P7 \\
\hline Conceicao & Carla & 5-O1, 5-P6, 5-P8 & Erickson & Bradley J & $3-\mathrm{O} 1$ \\
\hline Constantinescu & Cris S. & 1-P5, 1-P8 & Esposito & Giuseppe & 1-P59 \\
\hline Cools & Danae & $1-\mathrm{P} 28$ & Estades & Eduardo & 1-P30 \\
\hline Cordero & Efrain & $1-08$ & Fajardo Ramin & Lais & 1-P47 \\
\hline Cordero & Esteban & $1-\mathrm{O} 8$ & Falip & Merce & 1-P35 \\
\hline Correia & Ines & $5-\mathrm{P} 7$ & Farsaris & Dimosthenis & 1-P57 \\
\hline Cortes & Jesus Maria & $1-\mathrm{O} 15$ & Fazdlin Abdul & Nur & 1-P60 \\
\hline Costabile & Teresa & 1-O9, 1-P41, 1-P43 & Rahim & & \\
\hline Cotton & Francois & $3-\mathrm{P} 4$ & Federici & Margherita & $1-\mathrm{P} 49$ \\
\hline Crary & John F. & $1-\mathrm{O} 6$ & Felmban & Ghadah & 1-P8 \\
\hline Criscuolo & Stefania & $1-\mathrm{P} 45$ & Ferguson & Sherise Desiree & $1-\mathrm{O} 16$ \\
\hline Crockett & Matthew & 2-P8 & Fernandez-Coello & Alejandro & 1-P35 \\
\hline Cross & Justin & 4-P3 & Ferraciolli & Suely Fazio & $1-\mathrm{P} 47$ \\
\hline Cruz Martins & Ricardo & $5-\mathrm{O} 2$ & Filipe & Joao Pedro & 1-P37, 1-P51 \\
\hline Cuco & Antonio & $1-\mathrm{P} 22$ & Fior & Alberto & $2-\mathrm{O} 2$ \\
\hline Cucurell & David & 1-P35 & Fischer & Urs & $1-\mathrm{O} 12$ \\
\hline Cunha & Bruno & 5-O1, 5-P8 & Fischetti & Aldo & 1-P49 \\
\hline Cunha & Ines & $1-\mathrm{P} 46$ & Fitsiori & Aikaterini & $1-\mathrm{O} 1$ \\
\hline Cuocolo & Renato & $1-\mathrm{P} 43$ & Fontes Pinto & Ana Mafalda & $1-\mathrm{P} 24$ \\
\hline Da Costa Leite & Claudia & $1-\mathrm{P} 47$ & Dos Reis & & \\
\hline Da Silva Piteira & Ana Rita & 2-P7 & Fournier & Laure & 3-O2, 3-O3 \\
\hline Dahan & Ariel & 2-P1 & Fragata & Isabel & $\begin{array}{r}\text { 2-O2, 1-P29, } \\
\text { 1-P62, 2-P5 }\end{array}$ \\
\hline D'Arco & Felice & $4-\mathrm{O} 1$ & Frazatti Benez & Juliana & 1-P38 \\
\hline Das & Ravi K & $1-\mathrm{O} 2$ & Freitas & Pedro Melo & $5-\mathrm{P} 7$ \\
\hline Davidhi & Andjoli & 1-P55 & Freitas Goncalves & Joao Pedro & $1-\mathrm{P} 29$ \\
\hline De La Gala & Dario Herran & 1-P63 & Frendl & Ahita & 1-P31 \\
\hline De La Rosa & Ezequiel & 1-P17 & Fuentemilla & Lluis & 1-P35 \\
\hline De Lucas & Enrique Marco & 1-P63 & Fufezan & Otilia & 4-P1 \\
\hline De Siqueira Campos & Christiane Monteiro & 1-P38 & Futo & Claudia & 1-P31 \\
\hline De Sousa Costa & Rangel & 1-P36, 1-P39 & Gagliardi & Alessio & 1-O11, 5-O3, 2-P6 \\
\hline Deib & Gerard & 5-O4, 1-P65 & Galante Mulki & Maria Jose & 1-P63 \\
\hline Dekeyzer & Sven & 1-P28 & Gama Lobo & Goncalo & 1-P29, 1-P62 \\
\hline Delattre & Benedicte M. A. & 1-O3, 1-P66 & Gamboa Lattorre & Lina Fernanda & 4-P2 \\
\hline Dellepiane & Francesco & 1-P10, 1-P14 & Garcia & Rute & 3-P3 \\
\hline Denis & Golovin & $1-\mathrm{P} 21$ & Gatzonis & Stylianos & 1-P57 \\
\hline Deutschmann & Hannes & $1-\mathrm{P} 3$ & Geraldo & Ana Filipa & 4-P4 \\
\hline Di Napoli & Alberto & 1-P10, 1-P14 & Gerencser & Eszter & 1-P31 \\
\hline Diehn & Felix E. & $3-\mathrm{O} 1$ & Ghodasara & Satyam & $1-\mathrm{O} 10$ \\
\hline Dineen & Rob & $1-\mathrm{P} 5$ & Gil Oliveira & Tiago & $1-\mathrm{O} 6$ \\
\hline Dineen & Rob A. & 1-P8 & Giovansili & Lama & 3-P5 \\
\hline
\end{tabular}




\begin{tabular}{|c|c|c|c|c|c|}
\hline Giraldo & Saulo Molina & 4-P2 & Kaveh & Mahya & 1-P53 \\
\hline Gomes & Fabio & 5-P7 & Kim & Soung Yung & 3-P2 \\
\hline \multirow{3}{*}{$\begin{array}{l}\text { Gomes Ferreira } \\
\text { Marques } \\
\text { Gomez-Chiari }\end{array}$} & Diogo Andre & 2-P7, 3-P9 & Klarhoefer & Markus & 1-O17, 1-P58 \\
\hline & & & Kok & Hong Kuan & 2-P1 \\
\hline & Marta & 4-O2 & Kona & Matthew & 1-P4 \\
\hline Goodkin & Olivia & $1-\mathrm{O} 2$ & Korfias & Stephanos & 1-P57 \\
\hline Gralla & Jan & $1-\mathrm{O} 12$ & Kratimenos & Theodoros & 1-P57 \\
\hline Gravina & Michela & $1-09$ & Krayyem & Apollo & $1-\mathrm{O} 16$ \\
\hline Grieb & Dominik & 2-P9 & Kreiss & Adam & 1-P31 \\
\hline Guatta & Ramona & $1-\mathrm{O} 1$ & Kremer & Stephane & 3-P4 \\
\hline Guedes & Marcelo & 1-P54 & Krishnakumar & Deepa & 4-P3 \\
\hline Guillaume & Jessica & $1-\mathrm{O} 7$ & Kukull & Walter & 1-O6 \\
\hline $\begin{array}{l}\text { Guimaraes } \\
\text { Goncalves }\end{array}$ & Fabricio & $4-\mathrm{O} 1$ & Kulcsar & Zsolt & $\begin{array}{l}\text { 1-O14, 1-O17, } \\
\text { 1-P58, 1-P59 }\end{array}$ \\
\hline Habuza & Tetiana & $\begin{array}{l}\text { 1-P11, 1-P12, } \\
\text { 1-P13, 1-P15 }\end{array}$ & $\begin{array}{l}\text { Kumar } \\
\text { Dundamadappa }\end{array}$ & Sathish & $1-\mathrm{P} 4$ \\
\hline \multirow{2}{*}{$\begin{array}{l}\text { Hafidzah Rahim } \\
\text { Halim }\end{array}$} & Nurul & $1-\mathrm{P} 60$ & Kumar Ganesan & Prem & 3-P7 \\
\hline & $\begin{array}{l}\text { Khairulanuar } \\
\text { Saad@Abdul }\end{array}$ & $5-\mathrm{P} 2$ & Kurbatova & Tetiana & $\begin{array}{l}\text { 1-P11, 1-P12, } \\
\text { 1-P13, 1-P15 }\end{array}$ \\
\hline Hamann & Janne M. & $1-\mathrm{O} 12$ & Labayen & Fermin & $1-\mathrm{O} 15$ \\
\hline Harijan & Pooja & 4-P3 & Labrecque & Jeremy & $1-\mathrm{O} 13$ \\
\hline Hassler & Eva & $1-\mathrm{P} 3$ & Lakhani & Dharia & 1-P65 \\
\hline Hegarty & Aidan & 2-P8 & Lam & Chee Wah & $1-\mathrm{O} 13$ \\
\hline Held & Jeremia & 1-P59 & Lanzillo & Roberta & 1-O9, 1-P-41,1P-43 \\
\hline Held & Ulrike & $1-\mathrm{O} 12$ & Layman & Rick & $1-\mathrm{O} 16$ \\
\hline Heran & Francoise & 3-P4 & Leao & Miguel & 4-P4 \\
\hline Heraud & Alexandre & $3-\mathrm{O} 3$ & Lecler & Augustin & $\begin{array}{l}\text { 1-O7, 3-O2, 3-O3, } \\
\text { 3-P4, 3-P5 }\end{array}$ \\
\hline Herdan & Aviva & 3-P5 & Lee & Jessamyn & $1-\mathrm{P} 53$ \\
\hline Hernandez & Irene & $1-\mathrm{O} 8$ & Lee & Jung-Hyun & 3-P2 \\
\hline \multirow{3}{*}{$\begin{array}{l}\text { Hierro } \\
\text { Hiraoka } \\
\text { Hofmeister }\end{array}$} & Fatima & 1-P24, 3-P3 & Lelyuk & Vladimir G. & 1-O5, 1-P21 \\
\hline & Sayuki & $1-\mathrm{P} 6$ & Lemos Neves & Eduardo Jorge & 1-P36, 1-P39 \\
\hline & Jeremy & $\begin{array}{l}\text { 1-O1, 2-O1, } \\
\text { 2-O3, 2-P3 }\end{array}$ & $\begin{array}{l}\text { Filho } \\
\text { Lemoyne }\end{array}$ & Sabine & $1-\mathrm{P} 28$ \\
\hline Holodny & Andrei & 1-P10 & Lerman & Amir & $3-\mathrm{O} 1$ \\
\hline Hunink & Myriam & $1-\mathrm{O} 13$ & Lersy & Francois & $3-\mathrm{P} 4$ \\
\hline Husain Hakim & Shakir & $1-\mathrm{O} 17$ & $\mathrm{Li}$ & Jing & $1-\mathrm{O} 16$ \\
\hline Ilya & Gubskiy & 1-O5, 1-P21 & $\mathrm{Li}$ & Shan & 1-P64 \\
\hline Ince & Leyla & $5-\mathrm{P} 3$ & Liebo & Greta & $3-\mathrm{O} 1$ \\
\hline Iutaka & $\mathrm{T}$. & 1-P54 & Liew & Xiao Ching & 1-P16 \\
\hline Iuzzolino & Valentina & $1-\mathrm{P} 40$ & Lino & Joao & 3-P8 \\
\hline Jaimes Martinez & Luisa Fernanda & 4-P2 & Lisec & Ajda & $1-\mathrm{P} 26$ \\
\hline Januario & Goncalo & 1-P18 & Liu & Benjamin P. & 1-P7 \\
\hline Jardim Pereira & Daniela & 1-P44 & Ljubisavljevic & Milos & 1-P11, 1-P12, \\
\hline Jefic & Svjetlana & 1-P32 & & & 1-P13, 1-P15 \\
\hline Jimenez-Marin & Antonio & $1-\mathrm{O} 15$ & Loebel & Ulrike & $4-\mathrm{O} 1$ \\
\hline Jocic Jakubi & Bosanka & 4-P6 & Loevblad & Karl-Olof & 2-O1, 2-O3, \\
\hline Johnson & Jason Michael & $1-\mathrm{O} 16$ & & & 1-P20, 2-P3 \\
\hline Jorens & Philippe & 1-P28 & Lohaus & Niklas & $1-\mathrm{O} 12$ \\
\hline Kaesmacher & Johannes & $1-\mathrm{O} 12$ & Lopes Dos Reis & Joao & 2-O2, 2-P5 \\
\hline Kandemirli & Sedat G. & 3-P1 & Lorga & Tiago & 1-P22, 1-P23 \\
\hline Karelys Ng Wong & Yiken & 1-O8, 1-P33 & Lucignani & Martina & 1-P14 \\
\hline Katsaros & Vasileios K. & 1-P56, 1-P57 & Ludovichetti & Riccardo & 1-P66 \\
\hline & & & Luft & Andreas & 1-O12, 1-P59 \\
\hline
\end{tabular}




\begin{tabular}{|c|c|c|c|c|c|}
\hline Luksho & Daria & $\begin{array}{l}\text { 1-P11, 1-P12, } \\
\text { 1-P13, 1-P15 }\end{array}$ & $\begin{array}{l}\text { Napolitano } \\
\text { Nardi }\end{array}$ & $\begin{array}{l}\text { Antonio } \\
\text { Valentina }\end{array}$ & $\begin{array}{l}\text { 1-P10, 1-P14 } \\
3-\mathrm{O} 1\end{array}$ \\
\hline Machi & Paolo & 2-O1, 2-O3, 2-P3 & Nattan Portes Souza & Marcio & 5-P4 \\
\hline Machovitsch & Agnes & $1-\mathrm{P} 31$ & Naval-Baudin & Pablo & 1-P35 \\
\hline Madjidyar & Jawid & 1-O14, 1-P58 & Neidl Van Gorkom & Klaus & 1-P11, 1-P12, \\
\hline Maia & Carolina & $1-\mathrm{P} 46$ & & & 1-P13, 1-P15 \\
\hline Maingard & Julian & 2-P1 & Nemeth & Bence & 1-O14, 1-O17, \\
\hline Malaquias & Maria Joao & $5-\mathrm{O} 2$ & & & 1-P42, 1-P58, \\
\hline Manali & Chitre & 4-P3 & & & 1-P59 \\
\hline Mangel & Tunde & 1-P31 & Nomec $C$ octolo & Ligia & 3-P9 \\
\hline Mankad & Kshitij & $4-\mathrm{O} 1$ & Neves Castelao & Filipa Marla & 2-P7, 3-P9 \\
\hline Mantatzis & Michalis & $1-\mathrm{P} 27$ & Nicolay & Simon & 1-P28 \\
\hline Manzolini & Christian & $1-\mathrm{O} 12$ & Nikogosova & Anait K. & $\begin{array}{l}1-05 \\
2-02\end{array}$ \\
\hline Maric & Daliborka & $1-\mathrm{P} 32$ & Nunes & Ana Paiva & $\begin{array}{l}2-\mathrm{O} 2 \\
5-\mathrm{P} 7\end{array}$ \\
\hline Marinho Pinto & Eduarda Maria & 1-P37, 1-P51, 3-P8 & Nunes & Cesar & $\begin{array}{l}5-\mathrm{P} 7 \\
4-\mathrm{P} 4\end{array}$ \\
\hline Markovic & Ivana & 4-P6 & Nunes & Joana & $\begin{array}{l}\text { 4-P4, 4-Pl } \\
1-\mathrm{P} 50\end{array}$ \\
\hline Marques Gomes & Pedro Miguel & $3-\mathrm{P} 3$ & Nyilas & Nora Luca & $\begin{array}{l}1-\mathrm{P} 50 \\
5-\mathrm{P} 8\end{array}$ \\
\hline Marrone & Stefano & $1-09$ & U Hare & Alan & 2-P8 \\
\hline Martinez-Gutierrez & Endika & $1-\mathrm{O} 15$ & $\begin{array}{l}\text { Oliveira De } \\
\text { Queiroz }\end{array}$ & Thyago & 1-P19 \\
\hline Martinez-Saez & Elena & $1-\mathrm{O} 8$ & Ollitrault & Alexis & $3-\mathrm{P} 5$ \\
\hline Marussi & Victor Hugo & 1-P34 & Omar & Afif & 5-P1, 5P2 \\
\hline Mas Estelles & Fernando & 1-P55 & Ozturk & Onural & 1-P9 \\
\hline Masciocchi & Carlo & 2-P6 & Ozturk & Seda & 1-P9 \\
\hline Maurovich-Horvat & $\mathrm{Pal}$ & 1-P50 & Pagliei & Valeria & $1-\mathrm{O} 11$ \\
\hline Mazon-Monparler & Miguel & 1-P55 & Pais & Rui & $1-\mathrm{P} 46$ \\
\hline Medvedev & Olga & 4-P1 & Palma & Giuseppe & $1-\mathrm{P} 41$ \\
\hline Meglic & Jakob & 1-P26 & Papp & Aniko & 1-P31 \\
\hline Melber & Katharina & 2-P9 & Pareto & Deborah & $1-\mathrm{P} 25$ \\
\hline Meling & Torstein R. & 1-O1, 1-O3, & Parizel & Paul & $1-\mathrm{P} 28$ \\
\hline & & 1-P66, 2-P3 & Parker & Alasdair P. J. & 4-P3 \\
\hline Meltzer & Hal & 1-P65 & Parodi & Simona & 1-P49 \\
\hline Mendes Pinto & Catarina & 5-O2, 1-P51, 3P8 & Pasquini & Luca & 1-P10, 1-P14 \\
\hline $\begin{array}{l}\text { Michailovich } \\
\text { Rostovtsev }\end{array}$ & Dmitry & $1-P 1,1-P 2$ & Patino & Angelica & 1-P64 \\
\hline Michaiylovna & Yliay & 1-P1 & Pelechrini & Maria & 1-P57 \\
\hline Zabrodskaya & & & Pelechrini & Mary & 1-P56 \\
\hline Michels & Lars & 1-O14, 1-P42, 1-P59 & Pelegrini Cancela & Karolina & 1-P52, 1-P54, 1-P61 \\
\hline Miranda & Andre Miguel & 4-P4, 4-P7 & Pemberton & Hugh G & $1-\mathrm{O} 2$ \\
\hline Mital & Payal & 3-P7 & Penna & Simone & $1-\mathrm{O} 9$ \\
\hline Mock & Charles & $1-\mathrm{O} 6$ & Pereira Morimoto & Tauy & 1-P38 \\
\hline Mohan & Suyash & $1-\mathrm{O} 10$ & Pertici & Leonardo & $1-\mathrm{O} 11$ \\
\hline Mohd Zain & Norzaini Rose & 1-P60, 5-P1 & Pestana Barradas & Pedro Henrique & 1-P44, 5-P7 \\
\hline Monteiro Santos & Danielly Viana & 1-P34, 1-P38 & Peters & Soenke & 1-P7 \\
\hline Monti & Serena & 1-P41 & Petracca & Maria & 1-O9, 1-P40, \\
\hline Morais & Teresa & 1-P62 & & & 1-P-41,1P-43 \\
\hline Morais-Ribeiro & Rafaela & $1-06$ & Phyu & Poe & 4-P3 \\
\hline Moreira & Bruno & 3-P8 & Pimentel Silva & Ricardo & 2-P7 \\
\hline Mournet & Sandy & $1-\mathrm{O} 7$ & Pina & Sofia & 1-P37 \\
\hline Muchart & Jordi & $4-\mathrm{O} 2$ & Pinheiro Ferreira & Cesar Augusto & 4-O1 \\
\hline Muniandy & S.C. & $5-\mathrm{P} 1$ & $\begin{array}{l}\text { Alves } \\
\text { Pinto }\end{array}$ & Joana & 1-P46 \\
\hline Munuera & Josep & $4-\mathrm{O} 2$ & Pinto Rodrigues & Joao Victor & $\begin{array}{l}1-\mathrm{r} 40 \\
1-\mathrm{P} 34\end{array}$ \\
\hline Nakacima Terumi & Katia & $1-\mathrm{P} 47$ & Pires & Ricardo Joao & $\begin{array}{l}1-P 34 \\
1-P 44\end{array}$ \\
\hline Nan & Brian & $1-\mathrm{O} 16$ & & $\begin{array}{l}\text { Ricardo Joao } \\
\text { Gaspar }\end{array}$ & \\
\hline
\end{tabular}




\begin{tabular}{|c|c|c|c|c|c|}
\hline Pla & Albert & $1-\mathrm{P} 33$ & Sala-Padro & Jacint & 1-P35 \\
\hline Planells Alduvin & Mariana & $4-\mathrm{O} 2$ & Sansone & Carlo & $1-\mathrm{O} 9$ \\
\hline Podlasek & Anna & $1-\mathrm{P} 5$ & Santarosa & Corrado & $1-\mathrm{P} 20$ \\
\hline Poillon & Guillaume & $1-\mathrm{O} 7$ & Santos & Alexandra & $1-\mathrm{P} 22$ \\
\hline Pokhylevych & Halyna & $1-\mathrm{O} 16$ & Santos & Fatima & 4-P7 \\
\hline \multirow[t]{2}{*}{ Pontillo } & \multirow[t]{2}{*}{ Giuseppe } & \multirow{2}{*}{$\begin{array}{l}\text { 1-O9, 1-P40, } \\
\text { 1-P41, 1-P43 }\end{array}$} & Santos Freire & Ines & 1-P22, 1-P23 \\
\hline & & & Saraiva & Joao Anibal & 4-P5, 5-P5 \\
\hline Porto Teixeira & Livia & 1-P47 & Sarria & Silvana & $1-\mathrm{O} 8$ \\
\hline Power & Sarah & 2-P8 & \multirow[t]{2}{*}{ Savatovsky } & \multirow[t]{2}{*}{ Julien } & \multirow{2}{*}{$\begin{array}{l}\text { 1-O7, 3-O2, } \\
\text { 3-O3, 3-P5 }\end{array}$} \\
\hline Poza & Javier Garcia & $1-\mathrm{P} 63$ & & & \\
\hline Pozsar & Kinga & 1-P31 & Saverio & Pollice & $1-\mathrm{P} 7$ \\
\hline Procaci & Eduardo & 2-P4 & Savoldi & Filippo & 1-P45 \\
\hline Quarantelli & Mario & 1-O9, 1P-43 & Savran & Burcu & $5-\mathrm{P} 3$ \\
\hline \multirow{3}{*}{$\begin{array}{l}\text { Queiros De } \\
\text { Almeida Xavier } \\
\text { Quental }\end{array}$} & Sofia & 2-O2, 2-P5, 4-P5 & Schellingerhout & Dawid & $1-\mathrm{O} 16$ \\
\hline & \multirow{2}{*}{ Rita } & & Schlunz-Hendann & Martin & 2-P9 \\
\hline & & 4-P4 & Schroeder & Christophe & 3-P2 \\
\hline Quintas-Neves & Miguel & 1-O6, 5-P5 & \multirow{2}{*}{ Schubert } & \multirow[t]{2}{*}{ Tilman } & \multirow{2}{*}{$\begin{array}{l}\text { 1-O14, 1-O17, } \\
\text { 1-P58, 1-P59 }\end{array}$} \\
\hline Rahim & N.H. & $5-\mathrm{P} 1$ & & & \\
\hline Rakic & Mladen & 1-P17 & Scott & Francis & $4-\mathrm{P} 3$ \\
\hline Ramalingam & W V B S & 3-P7 & Sekhri Sethi & Kanika & 3-P7 \\
\hline Ramos & Cristina & 1-P51 & Sene & Thomas & $1-\mathrm{O} 7$ \\
\hline Ramos Neri & Laise & 1-P47 & Sequeira & Marta & 1-P62 \\
\hline Ramos-Usuga & Daniela & $1-\mathrm{O} 15$ & Serra & Maria Mercedes & 1-P30 \\
\hline Rashiwala & Abhi & $1-\mathrm{O} 16$ & Shakir Husain & Hakim & 1-P58 \\
\hline Raymand & Lee & 2-P2 & Shelmerdine & Susan & $1-\mathrm{P} 30$ \\
\hline Rebollo & Monica & $4-\mathrm{O} 2$ & Shibuya Setuguti & Mika & $5-\mathrm{P} 4$ \\
\hline Reis & Joao & 1-P62 & Silva & Carla & 2-P4 \\
\hline Reishofer & Gernot & 1-P3 & Sima & Diana & 1-P17 \\
\hline Reymond & Philippe & $1-\mathrm{P} 20$ & Simbrunner & Josef & 1-P3 \\
\hline Ribeiro & Joana & 1-P46 & Simiyu & Gillian Lylian & 1-P11, 1-P12, \\
\hline Ribeiro Santos & Mariana & 4-P5, 5-P5 & & & 1-P13, 1-P15 \\
\hline Riccio & Daniele & $1-\mathrm{O} 9$ & Simoes Da & Maria Ines & $1-\mathrm{P} 24$ \\
\hline Riccio & Eleonora & $1-\mathrm{P} 40$ & $\begin{array}{l}\text { Silva Prisco } \\
\text { Simst }\end{array}$ & & \\
\hline Rico Pons & Inmaculada & 1-P35 & Simsek & Onur & 5-P3 \\
\hline Rito Cruz & Luis & 2-P5 & Siri & Giacomo & 1-P49 \\
\hline Rocha & Sonia & 1-P24 & Smeets & Dirk & 1-P17 \\
\hline Rodrigues Rios & Ana Cristina & 2-P7, 3-P9 & Smetanina & Darya & $\begin{array}{l}\text { 1-P11, 1-P12, } \\
\text { 1-P13, 1-P15 }\end{array}$ \\
\hline Rohatgi & Saurabh & 1-P4 & Smits & Marion & $1-\mathrm{O} 13$ \\
\hline Rojano & Alba & $1-\mathrm{O} 8$ & Snoeckx & Annemiek & 1-P28 \\
\hline Romano & Andrea & 1-P10, 1-P14 & Soares & Pedro & $1-\mathrm{P} 22$ \\
\hline Romano & Nicola & 1-P49 & Soares-Fernandes & Joao & 4-P5 \\
\hline Rombold & Taylor & $5-\mathrm{O} 4$ & Soh & Calvin & 1-P48 \\
\hline Rosenberg & Martin & 1-P7 & Solymosi & Diana & 1-P31 \\
\hline Rosi & Andrea & 2-O1, 2-O3, 2-P3 & Sood & Neha & 3-P7 \\
\hline Rossello & Aleix & 1-P35 & Sousa & Ana Paula & $5-\mathrm{O} 2$ \\
\hline Rostovtseva & Tatiana & $1-\mathrm{O} 5$ & Sousa & Sergio & $1-\mathrm{P} 37$ \\
\hline Rovira & Alex & 1-O8, 1-P33 & Sousa Abreu & Vasco & 5-O2, 1-P37, 2-P4 \\
\hline Ruffinengo & Uberto Luigi & 1-P49 & Splendiani & Alessandra & 1-O11, 5-O3, 2-P6 \\
\hline Sa Da Silva & Jose Manuel & 1-P37 & Statsenko & Yauhen & 1-P11, 1-P12, \\
\hline Sa Pinto & Vasco & 2-P4 & & & 1-P13, 1-P15 \\
\hline Saatci & Ozlem & 3-P1 & Stefanatou & Maria & 1-P57 \\
\hline Sachdev & Sonal & 2-P1 & Stefanou & Danai-Eleni & 1-P56, 1-P57 \\
\hline
\end{tabular}




\begin{tabular}{|c|c|c|c|c|c|}
\hline Steigmiller & Klaus & $1-\mathrm{O} 12$ & Van Goethem & Johan & $1-\mathrm{P} 28$ \\
\hline Steketee & Rebecca M E & $1-\mathrm{O} 2$ & Vanden Bossche & Stephanie & $1-\mathrm{P} 28$ \\
\hline Stevenson & Rebecca & 1-P8 & Varallyay & Peter & 1-P31 \\
\hline Stranjalis & George & 1-P56 & Vargas Gomez & Maria Isabel & 1-O1, 1-O3, \\
\hline Studencnik & Fritz & 1-P3 & & & 1-P20, 1-P66 \\
\hline Sucapane & Patrizia & 2-P6 & Varrassi & Marco & $5-\mathrm{O} 3$ \\
\hline Sureshkumar & Anish & 1-P64 & Vaz Da Silva & Filipe & 2-P4 \\
\hline Surlan Popovic & Katarina & $1-\mathrm{P} 26$ & Vazgenovich & Harry & 1-P1 \\
\hline Suzuki & Lisa & $1-\mathrm{P} 47$ & $\begin{array}{l}\text { Papayan } \\
\text { Veiga }\end{array}$ & Marcos & 1-P62 \\
\hline Szalmas & Orsolya & 1-P31 & Ventura & Nina & 1-P36, 1-P39 \\
\hline Szirmai & Danuta & 1-P31 & Vercruyssen & Sophie & $1-\mathrm{P} 17$ \\
\hline Szolics & Miklos & 1-P11, 1-P12, & Verentzioti & Anastasia & $1-\mathrm{P} 57$ \\
\hline Szudi & Gabor & $\begin{array}{l}1-\mathrm{P} 13,1-\mathrm{P} 13 \\
1-\mathrm{P} 31\end{array}$ & Vernooij & Meike W & $1-\mathrm{O} 2$ \\
\hline Tagliente & Emanuela & $1-\mathrm{P} 14$ & Vernuccio & Federica & 1-P30 \\
\hline Takeshi Setuguti & Daniel & 5-P4 & Videira & Goncalo & 1-P37 \\
\hline Tan & Garryck & $1-\mathrm{P} 53$ & Vignal & Catherine & $1-\mathrm{O} 7$ \\
\hline Tarabishy & Abdul & $1-\mathrm{P} 65$ & Vila Real & Marta & 4-P4 \\
\hline Tardivo & Valentina & $1-\mathrm{P} 49$ & Vishnyakova & Anastasia & 1-O5, 1-P21 \\
\hline Tarrio & Joao & 1-P51 & Vos & Sjoerd B & $1-\mathrm{O} 2$ \\
\hline Tateishi & Hidekatsu & $1-\mathrm{P} 6$ & Vossough & Arastoo & 4-O1 \\
\hline Tavares Lucato & Leandro & $1-\mathrm{P} 47$ & Vranjes & Dalibor & 1-P32 \\
\hline Teixeiras & Sara & $4-\mathrm{O} 1$ & Wang & Ethan & $1-\mathrm{O} 16$ \\
\hline Tekcan Sanli & Deniz E. & 3-P1 & Wanke & Isabel & $2-\mathrm{O} 3$ \\
\hline Tench & Christopher & $1-\mathrm{P} 8$ & Wegener & Susanne & 1-O12, 1-P59 \\
\hline Teruel-Coll & Regina & 1-P55 & Weon & Young Cheol & 1-P7 \\
\hline Teunissen & Wouter & $1-\mathrm{O} 13$ & Westphal & Laura Philine & $1-\mathrm{O} 12$ \\
\hline Teylan & Merilee & $1-\mathrm{O} 6$ & Wiest & Roland & $1-\mathrm{O} 12$ \\
\hline Thornton & John & 2-P8 & Winklhofer & Sebastian & $1-\mathrm{O} 12,1-\mathrm{O} 14$ \\
\hline Thurner & Patrick & 1-O14, 1-P58, 1-P59 & Woo Park & Dong & 3-P6 \\
\hline To & Lau Hoi & $2-\mathrm{P} 2$ & Xavier & Joao & $5-\mathrm{O} 2,2-\mathrm{P} 4$ \\
\hline Tomais & Dimitrios & 1-P57 & Yildirim & Duzgun & 3-P1 \\
\hline Tommasino & Emanuele & 1-O11, 5-O3, 2-P6 & Yilmaz & Hasan & $2-\mathrm{O} 1$ \\
\hline Tortora & Mario & $1-\mathrm{P} 40,1-\mathrm{P} 41$ & Yim & Younghee & $1-\mathrm{O} 4$ \\
\hline Tranfa & Mario & 1-P40, 1-P41 & Yurievich & Rynda Artemii & 1-P1, 1-P2 \\
\hline Treyer & Valerie & $1-\mathrm{P} 42$ & Zaki & Lara A M & $1-\mathrm{O} 2$ \\
\hline Tsuchiya & Kazuhiro & 1-P6 & Zaki & Nazar & 1-P12 \\
\hline Turk & Sevcan & $1-\mathrm{P} 30$ & Zmuda & Mathieu & $3-\mathrm{O} 2,3-\mathrm{O} 3$ \\
\hline Uberoi & Anmol & $3-\mathrm{P} 7$ & Zuber & Kevin & $3-\mathrm{P} 5$ \\
\hline Udare & Ashlesha Satish & $1-\mathrm{P} 30$ & \multirow{5}{*}{\multicolumn{3}{|c|}{$\begin{array}{l}\text { Publisher's note Springer Nature remains neutral with regard to jurisdic- } \\
\text { tional claims in published maps and institutional affiliations. }\end{array}$}} \\
\hline Ugga & Lorenzo & $1-\mathrm{P} 43$ & & & \\
\hline Uzianbaeva & Liaisan & $\begin{array}{l}\text { 1-P11, 1-P12, } \\
\text { 1-P13, 1-P15 }\end{array}$ & & & \\
\hline Van Den Hauwe & Luc & $1-\mathrm{P} 28$ & & & \\
\hline Van Eyndhoven & Simon & $1-\mathrm{P} 17$ & & & \\
\hline
\end{tabular}

